\title{
Properties of Single Walled Carbon Nanotube Films
}

by

Shrividya Ravi

\author{
A thesis \\ submitted to the Victoria University of Wellington \\ in fulfilment of the \\ requirements for the degree of \\ Master of Science \\ in Physics.
}

Victoria University of Wellington

2008 



\begin{abstract}
The preparation and physical properties of transparent, single-walled carbon nanotube (SWNT) networks fabricated from a novel, organic dispersion are described here for the first time. Characterisation of SWNT dispersions uncovered shifts in the radial breathing modes as a function of aggregation. These modes were redshifted in centrifuged butylamine dispersions by $\sim 3 \mathrm{~cm}^{-1}$.

SWNT films cast using a simple, drop-deposition technique were annealed at $300{ }^{\circ} \mathrm{C}$ after fabrication to remove solvent and surfactant residue. Annealed films with a sheet resistance of magnitude $\sim 10^{4} \mathrm{k} \Omega / \square$ and transparency of $\sim 85 \%$ were fabricated in this study. The optoelectronic properties showed some inconsistency due to varying levels of oxygen doping and film thickness. Thin films annealed at $500{ }^{\circ} \mathrm{C}$ were found to be preferentially depleted of nanotubes with high chiral angle and small diameter. Oxidative effects were also observed upon annealing at temperatures as low as $300{ }^{\circ} \mathrm{C}$. However, the reasons for this premature combustion are as yet uncertain.
\end{abstract}

Temperature-dependent conduction studies revealed that the removal of adsorbed surfactant considerably reduced tunnelling barriers in annealed films. The dominant conduction mechanism in both unannealed and annealed films was found to be $3 \mathrm{D}$ variable range hopping. In the annealed films, a high temperature activation regime (with activation energy of $220 \mathrm{meV}$ ) was observed above $225 \mathrm{~K}$. This regime is due to thermal activation over Schottky barriers within the nanotube network or electron activation over the pseudogap in armchair tubes. 


\section{Acknowledgments}

First and foremost, I would like to thank my supervisors: Prof. Alan Kaiser and Dr. Chris Bumby for their valuable guidance and support. I am grateful to Prof. Pablo Etchegoin and Matthias Meyer for helping me with the Raman spectrometer. A big thanks to David Flynn who took care of all the technicalities of the SEM. Another round of thanks goes to Dr. Ben Ruck and Andrew Preston for helping me with the conductivity measurements on the glass cryostat. I would like to also acknowledge the fellow residents of the VUW basement and my hacky and soccer mates for all the good times outside the lab. Finally, a giant thanks to my family and Dmitri for their unconditional support. 


\section{Contents}

1 Introduction $\quad 13$

2 Background Theory 17

2.1 SWNTs: Structure and Properties . . . . . . . . . . . . . . 17

2.1.1 Geometric structure . . . . . . . . . . . 17

2.1.2 Electronic properties . . . . . . . . . . . . . 18

2.2 Raman spectroscopy . . . . . . . . . . . . . . 23

2.2.1 The G-mode . . . . . . . . . . . . . 26

2.2.2 Double resonance peaks: The D-mode and G'-mode . . . . 28

2.2.3 Radial breathing modes . . . . . . . . . . . . . 30

2.3 Conduction in SWNT networks . . . . . . . . . . . . . 38

2.3.1 Conduction mechanisms ............. . 40

2.3.2 Fluctuation induced tunnelling (FIT) . . . . . . . . . . . 40

2.3.3 Variable range hopping . . . . . . . . . . . . . . . . 42

3 Characterisation techniques $\quad 49$

3.1 Basic physical characterisation . . . . . . . . . . . . 50

3.1.1 Optical transparency ............... 50

3.1.2 Measuring conductance ............. 50

3.1.3 Surface morphology . . . . . . . . . . . . . . 52

3.2 Post-deposition treatment . . . . . . . . . . . . 53

3.2.1 Thermogravimetric analysis ... . . . . . . . . 53 
3.2 .2 Annealing .................... . . 54

3.3 Raman spectroscopy . . . . . . . . . . . . . . . 55

3.3.1 The Raman spectrometer ............. 55

3.3 .2 Confocal microscopy . . . . . . . . . . . . . 57

3.3.3 Solution samples . . . . . . . . . . . . . . 59

3.3.4 Solid samples . . . . . . . . . . . . . . . 60

3.4 Low temperature conductivity . . . . . . . . . . . . . . . 60

3.4.1 Closed-cycle cryostat . . . . . . . . . . . 60

3.4.2 Glass cryostat . . . . . . . . . . . . 62

$\begin{array}{lll}4 & \text { Sample preparation } & 65\end{array}$

4.1 Dispersion ......................... 65

4.1.1 The dispersion process . . . . . . . . . . . . 67

4.1.2 Surfactants in SWNT dispersions . . . . . . . . . . 68

4.1.3 The dispersion procedure . . . . . . . . . . . . . . 70

4.2 Thin film fabrication . . . . . . . . . . . . . . . . . 72

4.2.1 Film deposition . . . . . . . . . . . . . 72

4.2.2 Improvements to optoelectronic properties . . . . . . . . 74

4.3 Conclusion . . . . . . . . . . . . . . . . . . 81

5 Raman characterisation $\quad 83$

5.1 SWNT dispersions . . . . . . . . . . . . . . . . 84

5.1.1 Surfactant selectivity .............. 86

5.1.2 Unbundling effects ................. 93

5.1.3 Raman mode shifts ................ 99

5.2 Thin films of SWNTs . . . . . . . . . . . . . . . 108

5.2.1 Annealing effects . . . . . . . . . . . . . . 110

5.3 Conclusion . . . . . . . . . . . . . . . . . 114 
6 Conduction Mechanisms in SWNT networks

6.1 Unannealed samples . . . . . . . . . . . . . . . 117

6.2 Annealed Samples . . . . . . . . . . . . . . . . . . 123

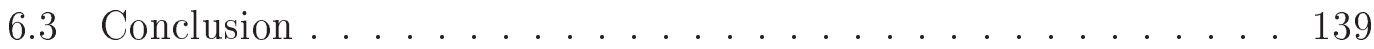

$\begin{array}{lll}7 & \text { Conclusion } & 141\end{array}$

Appendix:

A LabView program for closed-cycle cryostat

B LabView program for glass cryostat 


\section{List of Figures}

1.1 Optoelectronic properties of SWNT films compared to ITO. . . . 15

2.1 Rolling of a graphene sheet into a nanotube. . . . . . . . . . . . . 19

2.2 The Brillouin zone of graphene. . . . . . . . . . . . . . . . . . . 19

2.3 Quantisation of the Brillouin zone in nanotubes. . . . . . . . . . 20

2.4 Van Hove singularities in nanotubes of different electronic type. . 21

2.5 The symmetrical stretching mode of $\mathrm{CO}_{2}$. . . . . . . . . . . 25

2.6 Raman spectrum of a SWNT sample. . . . . . . . . . . . . 26

2.7 The LO and TO vibrational modes of the G-band. . . . . . . . . . 26

2.8 G-mode lineshape for metallic and semiconducting tubes. . . . . . 28

2.9 Diameter and electronic dependence the $\mathrm{G}^{-}$mode. . . . . . . . . 29

2.10 RBM spectra for isolated and bundled samples. . . . . . . . . . . 30

2.11 The change in RBM frequency as a function of bundle size. . . . . 32

2.12 A Kataura plot of $\mathrm{E}_{i i}$ transition vs. wavenumber. . . . . . . . . 33

$2.13 \mathrm{E}_{i i}$ transitions in bundled and isolated SWNTs. . . . . . . . . . 36

2.14 The effects of morphology on RBM frequency and intensity. . . . 37

2.15 The pickup stick model for nanotubes. . . . . . . . . . . . . . 38

2.16 Reduction in junction resistance by adjusting tube geometry. . . . 39

2.17 The energy barrier between two conducting segments. . . . . . . . 41

2.18 Localised wavefunction of a lattice defect. . . . . . . . . . . 43

2.19 Density of states for a pure and partially disordered semiconductor. 43 
2.20 Ion core potentials in a crystalline and amorphous material. . . . 44

3.1 Schematic of the physical characterisation performed on SWNT dispersions and thin films. . . . . . . . . . . . . . . . . 49

3.2 Edwards E306A thermal evaporator . . . . . . . . . . . . 51

3.3 4-boat insert for multiple metal evaporation. . . . . . . . . . 51

3.4 The SDTQ600 DSC/TGA instrument. . . . . . . . . 53

3.5 Radiative annealing oven . . . . . . . . . . . . . 55

3.6 A schematic of the Jobin Yvon LabRam confocal Raman spectrometer used to obtain Raman spectra. . . . . . . . . . . 56

3.7 The holographic notch filter. . . . . . . . . . . 56

3.8 Spatial selection with confocal microscopy. . . . . . . . . . . 58

3.9 Variation in numerical aperture with angle $\mu . \ldots . . . . .58$

3.10 Airy disks for different numerical apertures. . . . . . . . . . . . 59

3.11 The experimental setup for collecting spectra from solution. . . . 59

3.12 A schematic of the cold finger of the closed-cycle cryostat . . . . 61

3.13 A schematic of the glass cryostat. . . . . . . . . . . 62

3.14 Diagram showing the front and back sides of the sample mount of the glass cryostat. . . . . . . . . . . . . . 63

3.15 Equivalent circuit diagram for obtaining conductance data at constant bias. . . . . . . . . . . . . . . . . 64

4.1 Surfactant micelles in water. . . . . . . . . . . . 66

4.2 The unzipping of nanotubes during sonication. . . . . . . . . . 67

4.3 7:1 THF:butylamine dispersions prepared with different nanotube concentrations. ......................... 71

4.4 Schematic of the hydrogen bonding between butylamine and THF. 72

4.5 Optoelectronic properties of unannealed SWNT networks. . . . . 73

4.6 SEM image of an unannealed film. . . . . . . . . . . . . . 74 
4.7 Differential thermogram showing the peak associated with evaporation of water. The two smaller peaks at $66^{\circ} \mathrm{C}$ and $80{ }^{\circ} \mathrm{C}$ are due to evaporation of THF and butylamine. . . . . . . . . . . . 75

4.8 Differential thermogram showing the two smaller peaks at $267^{\circ} \mathrm{C}$ and $439{ }^{\circ} \mathrm{C} \ldots \ldots \ldots \ldots \ldots \ldots$

4.9 SEM image of a SWNT film annealed at $140{ }^{\circ} \mathrm{C} \ldots \ldots . . . . . .76$

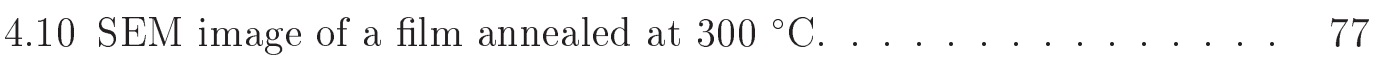

4.11 Improvement in the conductance after successive drop-casting and annealing. .............................. 78

4.12 Optoelectronic properties of annealed SWNT networks. . . . . . . 79

4.13 Optoelectronic properties after successive drop-casting and annealing. ....................... 80

4.14 SEM image of a thin film annealed at $500{ }^{\circ} \mathrm{C}$. . . . . . . . . . 80

5.1 Spectra of raw HiPCO powder and a SWNT dispersion prepared with a 7:1 ratio of THF:butylamine taken under the $514.5 \mathrm{~nm}$ laser. 85

5.2 Spectra of raw HiPCO powder and a SWNT dispersion prepared with a 7:1 ratio of THF:butylamine taken under the $632.8 \mathrm{~nm}$ laser. 85

5.3 The process for studying selectivity in SWNT-butylamine dispersions 88

5.4 Raman spectra of two sonicated and centrifuged dispersions obtained with the $632.8 \mathrm{~nm}$ laser. . . . . . . . . . . . . . . . 89

5.5 Raman spectra of two sonicated and centrifuged dispersions obtained with the $514.5 \mathrm{~nm}$ laser. . . . . . . . . . . . . 89

5.6 Division by electronic type of a RBM spectrum. . . . . . . . . . . 90

5.7 Labelled screenshot of a QRAP fitting session. . . . . . . . . . . . 91

5.8 Kataura plots showing the resonance window in samples with different levels of aggregation. . . . . . . . . . . . . 95

5.9 Raman spectra obtained with the $632.8 \mathrm{~nm}$ laser line for four solutions prepared with different THF:butylamine ratio. . . . . . 98 
5.10 Raman spectra obtained with the $514.5 \mathrm{~nm}$ laser line for four solutions prepared with different THF:butylamine ratio. . . . . . 98

5.11 RBM shift between the centrifuged amine dispersion and raw powder 102

5.12 RBM shift between the SDS dispersion and raw powder . . . . . . 102

5.13 RBM shifts between the precipitate and raw powder . . . . . . . 103

5.14 RBM shifts between an unannealed film and raw powder . . . . . 104

5.15 Plot of intensity vs. frequency for the $\mathrm{G}^{+}$peak. . . . . . . . . 106

5.16 Linewidth or FWHM vs. frequency for the $\mathrm{G}^{+}$peak. . . . . . . . 107

5.17 Frequency of the $\mathrm{G}^{+}$mode compared to the $\mathrm{G}^{-}$mode. . . . . . . 107

5.18 Summary of the Raman shifts in various samples compared to the raw HiPCO powder. . . . . . . . . . . . . . . . 108

5.19 RBM spectra from five spots on an unannealed network taken under the $632.8 \mathrm{~nm}$ laser. . . . . . . . . . . . . . . 109

5.20 Raman spectra of annealed films under the $632.8 \mathrm{~nm}$ laser. . . . . 110

5.21 Raman spectra of annealed films under the $514.5 \mathrm{~nm}$ laser. . . . . 110

5.22 RBM shifts between unannealed and annealed films. . . . . . . . . 112

5.23 Diameter vs. chiral angle plot for films annealed to $300{ }^{\circ} \mathrm{C}$ and

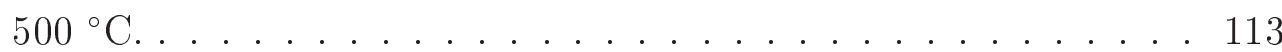

6.1 SEM images of unannealed and annealed SWNT films. . . . . . . 116

6.2 Homogeneous and cracked unannealed SWNT films. . . . . . . . . 116

6.3 A comparison of different VRH mechanisms for U1. . . . . . . . . 118

6.4 A comparison of different VRH mechanisms for U2. . . . . . . . . 119

6.5 A comparison of different VRH mechanisms for U3. . . . . . . . 120

6.6 Normalised conductance vs. temperature for the unannealed samples. . . . . . . . . . . . . . . . . . . . 121

6.7 Graph of normalised conductance for all annealed samples. . . . . 124

6.8 The full temperature range for sample s3. . . . . . . . . . . . . 124 
6.9 A comparison of 2D VRH, 3D VRH and 3D VRH with electronelectron interaction mechanisms for s3. . . . . . . . . . . 125

6.10 The effect of a subtracted shunt conductance. . . . . . . . . . . . 126

6.11 Normalised conductance vs. temperature for s3. . . . . . . . . . . 128

6.12 Graph showing the fitting results of two different dual-regime VRH models. ....................... 129

6.13 The combined fits including activation for the two VRH models. . 132

6.14 Percentage error between the fitted and raw data. . . . . . . . . . 132

6.15 Contribution of the conduction models at different temperatures. . 133

6.16 Conduction processes described by model 2 within s3. . . . . . . 133

6.17 Temperature-dependent conductance curve for s4 . . . . . . . . 135

6.18 Normalised conductance for samples s4 and U3. . . . . . . . . 136

6.19 Normalised conductance vs. temperature for samples s1 and s2 at different applied voltages. . . . . . . . . . . . . 137

6.20 Plot of the number of carrier electrons at different bandgap energies.138

7.1 General summary of results presented in Chapters 5 and 6. . . . . 141

7.2 SEM images of SWNT films annealed at various temperatures. . . 142

7.3 Effects of annealing on optoelectronic properties. . . . . . . . . 143

7.4 Change in sheet resistance for the same optical transparency in films enriched in metallic SWNTs. . . . . . . . . . . . . . . . 144

7.5 Increase in optoelectronic properties and decrease in localisation length upon annealing. . . . . . . . . . . . . . . 144

A.1 Front panel screenshot of the LabView program used to set the target temperature. . . . . . . . . . . . . . . . . . 148

A.2 The plot of temperature vs. time displayed in the 'time to stability'

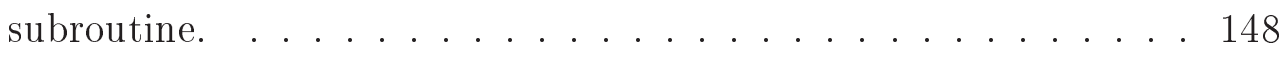

B.1 Serial polling of the four digital multimeters. . . . . . . . . . . . . 152 
B.2 Front panel screenshot of the LabView program used with the glass cryostat. . . . . . . . . . . . . . . . 152 


\section{List of Tables}

2.1 Experimental values of the four G-band modes for semiconducting SWNTs in a bundled sample. . . . . . . . . . . . . 27

4.1 Values of the physical parameters that determine miscibility for butylamine and THF. . . . . . . . . . . . . 72

5.1 The relative fractions of metallic and semiconducting tubes in solution and powder. ...................... 92

5.2 Shifts in the $\mathrm{E}_{i i}$ transitions between raw powder and butylamineSWNT dispersions. . . . . . . . . . . . . . . . 95

5.3 Details of the predominant RBMs observed under the $632.8 \mathrm{~nm}$ and $514.5 \mathrm{~nm}$ laser lines. . . . . . . . . . . . . . . . . 97

5.4 Description of the dispersions used to understand the efficacy of butylamine. . . . . . . . . . . . . . . . . 99

5.5 Sample description and the number of spectra used to obtain an average for each RBM peak. . . . . . . . . . . . . . . . . 101

6.1 Abbreviations and descriptions of unannealed samples. . . . . . . 118

6.2 Parameters for U1 and U3 extracted from a 3D VRH fit. . . . . . 122

6.3 Parameters for the two dual-regime VRH models depicted in Figure $6.12 \ldots \ldots \ldots \ldots \ldots$

6.4 Activation parameters for fits shown in Figure 6.13. . . . . . . . 131 
6.5 Parameters from the 3D VRH fit for annealed and unannealed samples prepared during the initial stages of MSc. . . . . . . . 136 


\section{Chapter 1}

\section{Introduction}

Novel nanomaterials are extensively sought after for new generation electronics. Macroscopic devices made from these materials can be assembled using bottomup techniques which allow tight control over morphology and scaling. The most prominent among current nanomaterials are carbon nanotubes. Since the widelyacknowledged discovery by Iijima [1] in $1991^{1}$, carbon nanotube research has developed into a fully-fledged field in its own right.

The wide appeal of carbon nanotubes, particularly single-walled nanotubes (SWNTs) lies in their phenomenal physical properties. Bundles (or ropes) of SWNTs have low resistivity $\left(\sim 10^{-4} \Omega-\mathrm{cm}\right)[4]$ and breaking strengths up to 52 GPa [5]. Due to this high tensile strength, SWNTs can be assembled into macroscopic networks that can take on a variety of morphologies. An inter-connected network of highly conducting wires can be deposited with low spatial coverage leading to high transparency coupled with a low sheet resistance.

Conducting networks fabricated from SWNTs are used in several technological applications such as transparent heaters [6] and thin film transistors (TFTs) [7]. They are also considered to be promising candidates to replace the expensive

\footnotetext{
${ }^{1}$ However evidence of carbon nanotubes had been published as early as 1952 [2, 3]
} 
but widely-used transparent conductor: indium tin oxide (ITO). SWNT films have been successfully demonstrated as transparent electrodes in organic light emitting diodes (OLEDs) [8] and solar cells [9, 10]. Recently, e-paper displays using carbon nanotube electrodes were demonstrated by Samsung [11].

The primary step in most current methods for fabricating SWNT networks involves dispersing the nanotubes in a solvent and surfactant. These solutions not only segregate clumps of nanotubes but they also disperse the unbundled nanotubes homogeneously in solution. Films can be cast from solution using a variety of techniques $[12,13,14,8]$. However, even the most efficient film deposition processes are time consuming since they utilise suspensions with low volatility. Films prepared from these dispersions require extensive post-deposition treatment to remove both the solvent and the surfactant. One of the motivations behind this study was to investigate alternative, less intensive routes for fabricating SWNT films. In this study, we introduce a novel methodology that uses a volatile organic dispersion and a simple, drop-deposition technique for fabricating SWNT networks.

Organic dispersions can exfoliate nanotubes from large clumps to small bundles or individual strands. Several groups have shown alkylamines to be particularly effective in this regard [15, 16, 17]. Furthermore, Maeda et al. have demonstrated enrichment of metallic SWNTs in dispersions of tetrahydrofuran (THF) and propylamine or octylamine $[18,19]$. Thin films deposited from these enriched fractions have a much higher conductivity for the same optical transparency than films deposited from non-enriched fractions [20].

In this study, we investigated nanotube dispersions prepared with butylamine and THF. Since this system has not been studied as yet, we address the prevalent 


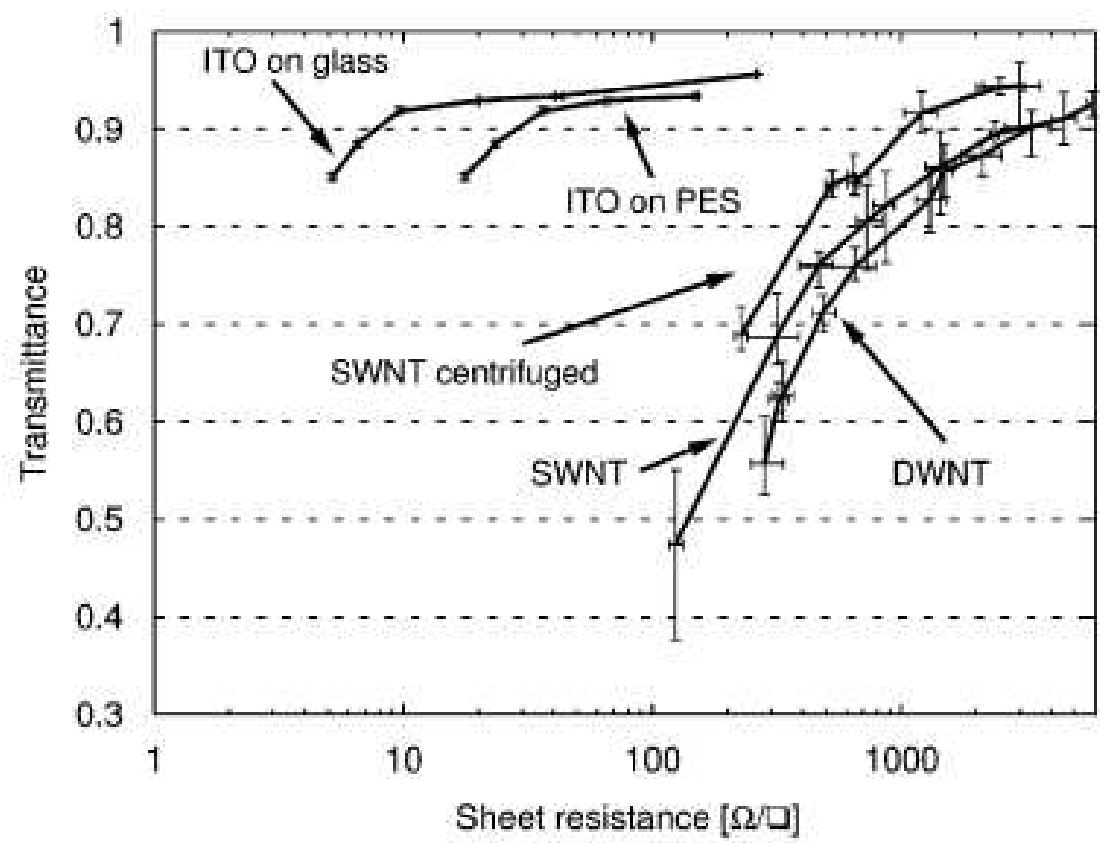

Figure 1.1: Optoelectronic properties of SWNT films compared to ITO [23].

issues for nanotube dispersions such as dispersion efficiency, electronic selectivity and the butylamine-nanotube interaction.

Despite the promise of SWNT thin films as transparent conductors, their optoelectronic properties are still far from ITO. Figure 1.1 shows the optoelectronic properties of SWNT thin films compared to ITO as of 2007. Many groups have reported improved sheet conductance after the addition of dopants [21] or extensive post-deposition treatments [22]. However, these studies relied on simple optoelectronic measurements such as optical transparency and DC sheet conductance.

The second focus of this thesis is a study of conduction in nanotube networks. Temperature-dependent conductivity studies from $300 \mathrm{~K}$ to $\sim 1 \mathrm{~K}$ were undertaken to gain insight into the mechanism of electron transport within SWNT networks. Understanding conduction at the nanoscale can be used to isolate the 
limitations of conductivity in SWNT networks. This knowledge can be applied to advance their conduction properties by devising better fabrication techniques or appropriate post-deposition treatments.

\section{Roadmap}

The second chapter of the thesis is comprised of background theory relevant to the topics covered in this study. The chapter opens with theory relating to the electronic properties of SWNTs. It is followed by a section covering Raman spectroscopy and how it can be used to understand the qualities of nanotube dispersions and films. The last section of the theory chapter details electron transport mechanisms within SWNT networks. The third chapter describes the various experimental characterisation techniques used in this study.

The fourth chapter covers the process of thin film fabrication. The relevant theory, literature review and experimental procedures pertaining to sample preparation are also included in this chapter. The effects of physical and chemical treatments carried out during sample preparation are analysed using Raman spectroscopy in Chapter 5. The last chapter presents the results of conduction studies performed on SWNT films. The thesis is concluded with a brief chapter highlighting the major findings. 


\section{Chapter 2}

\section{Background Theory}

\subsection{SWNTs: Structure and Properties}

\subsubsection{Geometric structure}

There is an intimate relationship between the structure of a nanotube and its electronic properties. This section demonstrates how simple structural information for any given SWNT can provide a detailed description of its electronic nature.

If we were to set about making SWNTs in a crude, mechanical fashion, we can deconstruct the process as shown in Figure 2.1. A unique assignment known as the 'chiral index' is used to quantify the length of the lattice vectors $\mathbf{a}_{1}$ and $\mathbf{a}_{2}$. The chiral vector $(\mathbf{R})$ results from a linear combination of the lattice vectors and is written as:

$$
\mathbf{R}=n \mathbf{a}_{1}+m \mathbf{a}_{2}
$$

where the integers $n$ and $m$ make up the chiral index. By convention, the index is usually represented as $(n, m)$. Since the chiral vector is equivalent to the circumference of the nanotube, a simple relation can be derived for the nanotube 
diameter using the chiral index:

$$
d=\frac{|\mathbf{R}|}{2 \pi}=\frac{a}{2 \pi} \sqrt{n^{2}+m^{2}+n m}
$$

where $a$ is the graphene lattice spacing that is equivalent to $\sqrt{3} d_{C-C}$ and $d_{C-C}$ is the bond length for $\mathrm{sp}^{2}$ carbon. Similarly, the chiral angle can be written as:

$$
\theta_{C}=\frac{\mathbf{a}_{1} \cdot \mathbf{R}}{\left|\mathbf{a}_{1}\right||\mathbf{R}|}=\tan ^{-1}\left(\frac{\sqrt{3} n}{2 n+m}\right)
$$

The chiral angle is an important parameter that gives valuable information regarding tube chirality and chemical reactivity (see Section 4.1). Due to the six-fold rotational symmetry of the hexagonal lattice, $\theta_{C}$ can only vary from $0^{\circ}$ to $30^{\circ}$. This variation gives rise to two types of tubes: chiral and achiral. As their name suggests, achiral tubes have no chirality. Hence, their reflection is identical to the original. Both the $(n, 0)$ and $(n, n)$ tubes are achiral and are usually referred to as zig-zag and armchair respectively due to the shape of C-C boundary at the reflection plane. Chiral tubes comprise the remaining set of nanotubes where $0^{\circ}<\theta_{C}<30^{\circ}$.

\subsubsection{Electronic properties}

In the earlier section we saw that SWNTs can be mechanically formed from a graphene sheet. Similarly, we can extrapolate the electronic structure from that of graphene using a zone folding method since most of the interesting electronic properties stem from the rolled-up graphene sheet. The capped end of the nanotubes - a semi-fullerene - plays a negligible role.

The semi-metallic nature of graphene can be seen from the Brillouin zone. The conduction and valence bands only cross at the edges of the first Brillouin 


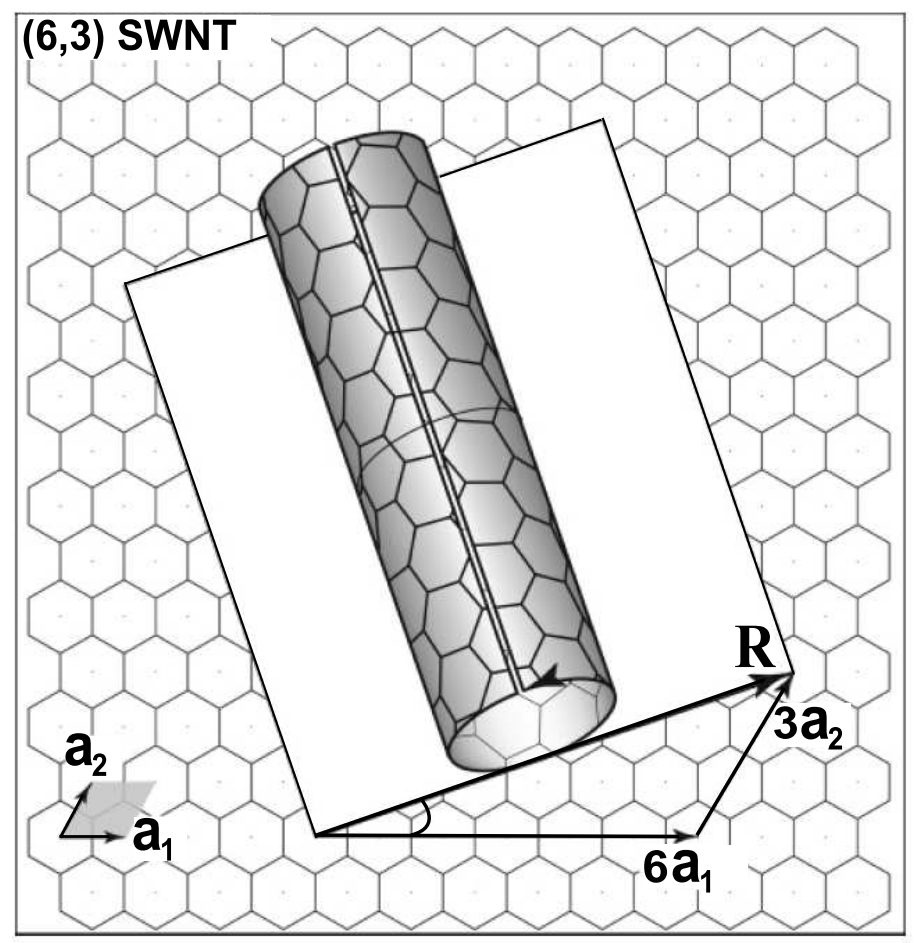

Figure 2.1: This schematic shows how a graphene sheet is cut according to the chiral vector $\mathbf{R}$ and rolled into a nanotube [24].
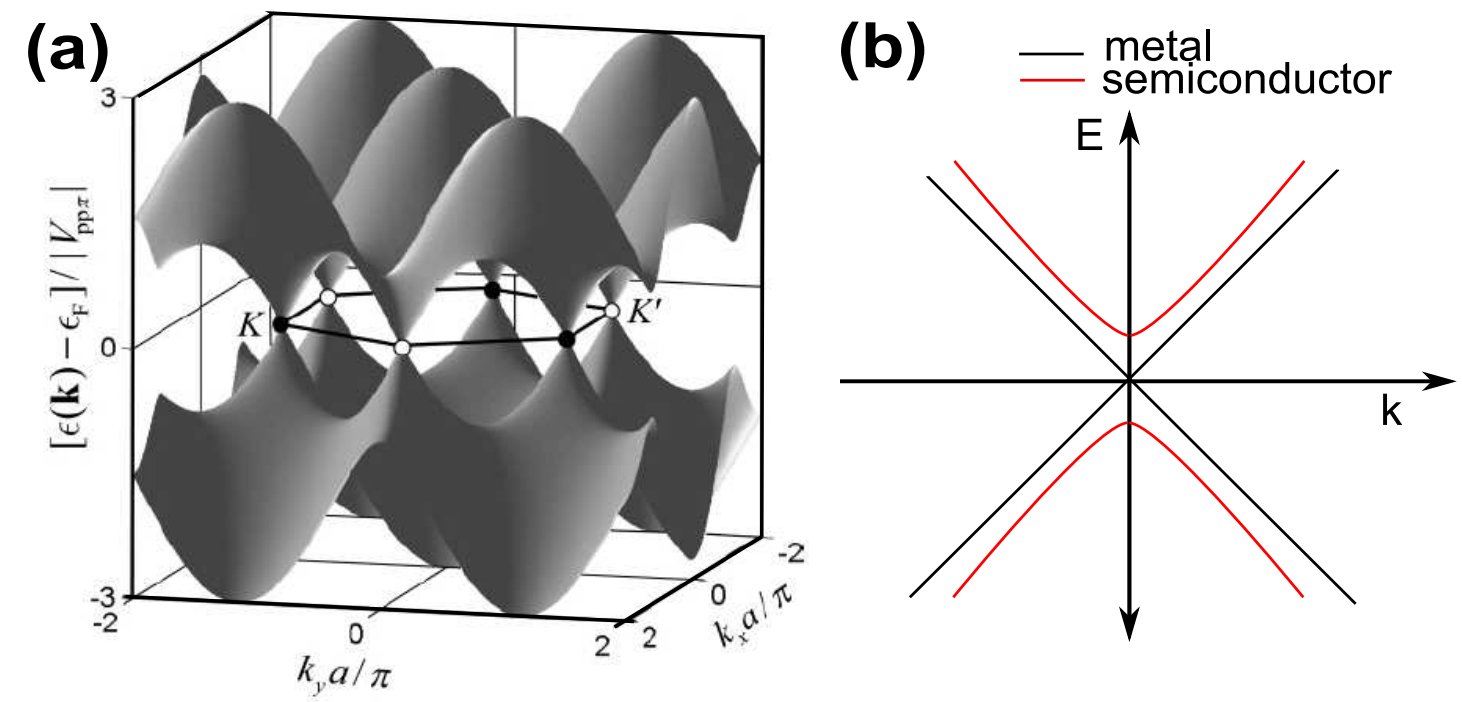

Figure 2.2: (a) The crossing of the conduction and valence bands only occurs at the K point of the Brillouin zone for graphene [24]. (b) A reduced depiction of the conduction and valence bands at and away from the K-point.

zone at the $\mathrm{K}$ point as shown by the black curve of Figure 2.2. At all other points, the bands do not cross and the material is semiconducting (the red curve in Figure 2.2). 


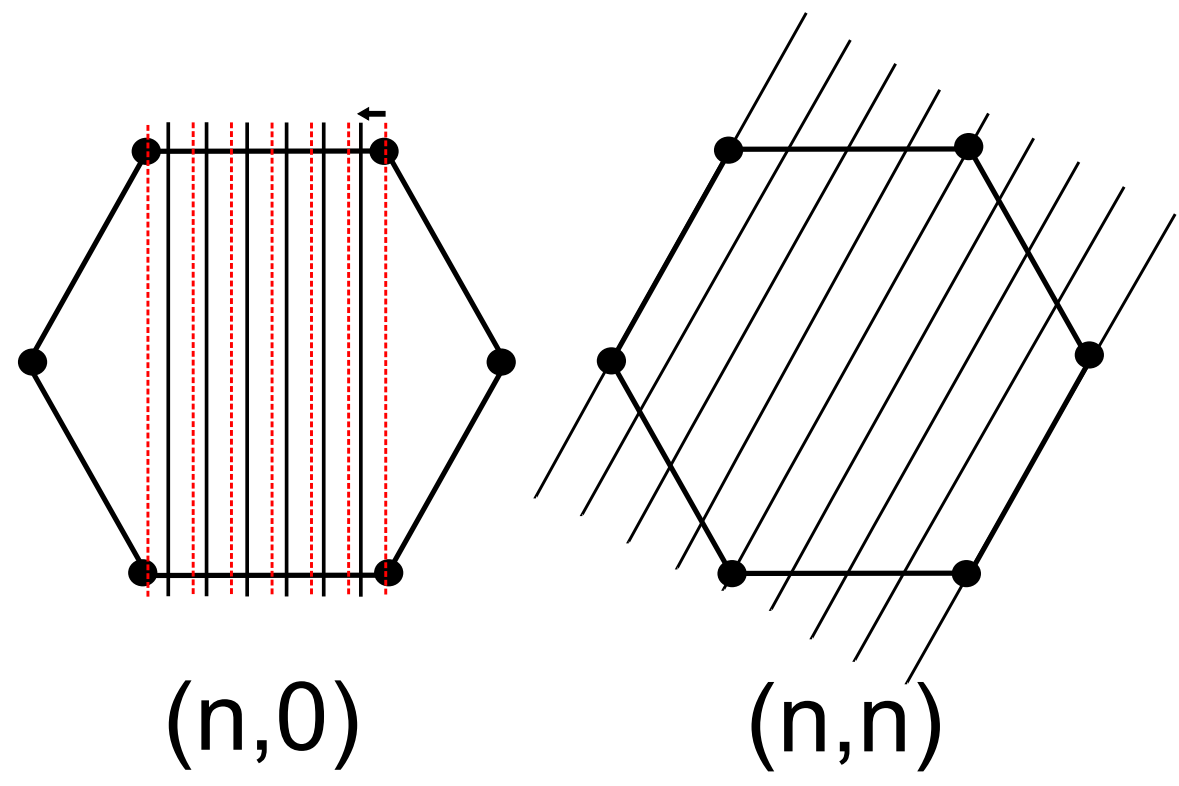

Figure 2.3: The quantised wavevectors for metallic $(n, 0)$ and $(n, n)$ tubes. The $\mathrm{K}$ points lie on the allowed sets of wavevectors for the $(n, n)$ nanotube indicating metallicity. However, a shift in the wavevectors due to curvature causes the $(n, 0)$ tubes to become non-metallic.

SWNTs are a 1D material and in order to extract an electronic structure from that of graphene, the circumferential dimension is quantised according to:

$$
\mathbf{k} \cdot \mathbf{R}=2 \pi q \quad(q=1,2,3,4 . . N)
$$

This quantisation reduces the Brillouin zone to a set of lines as shown in Figure 2.3. Nanotubes with wavevectors that include the K point are metallic. Since the $K$ point occurs at $\left(\mathbf{k}_{1}-\mathbf{k}_{2}\right) / \mathbf{3}$ the set of nanotubes where $(n-m)=3 q ;(n, n)$ and $(\mathrm{n}, 0)$ are also metallic.

The electronic nature of the SWNTs can also be seen from the density of states (DOS) at the Fermi level (blue and black curves in Figure 2.4). The DOS for a semiconducting tube is zero around the Fermi level indicating a bandgap. The bandgap is of the order of $1 \mathrm{eV}$ though it varies inversely with nanotube diameter. Electronic transitions for SWNTs are sharp, discrete excitations be- 


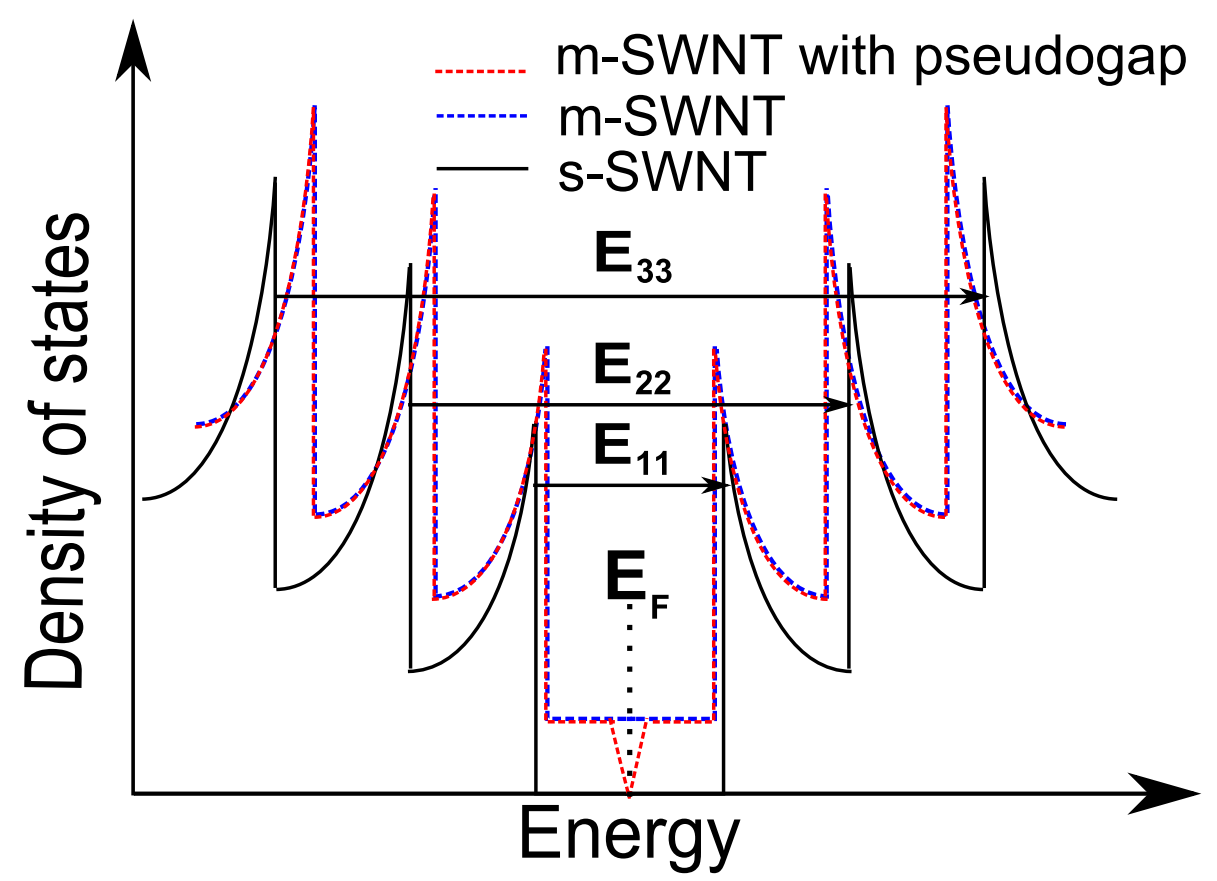

Figure 2.4: A qualitative schematic of the density of states for various SWNTs. The black and blue curves show the DOS for a semiconducting (s-SWNT) and metallic (m-SWNT) nanotube respectively while the red curve shows the DOS for a quasi-metallic zig-zag tube or an armchair tube within a bundle.

tween the Van Hove Singularities (VHS). The transitions are labelled $\mathrm{E}_{i i}$ where $\mathrm{i}$ $\geq 1$ (see Figure 2.4). $\mathrm{E}_{i i}$ transitions have been shown to dominate $\mathrm{E}_{i j}$ transitions where $\mathrm{i} \neq \mathrm{j}[25]$. Another labelling convention is the inclusion of ' $\mathrm{M}$ ' or ' $\mathrm{S}$ ' as a superscript to indicate the nanotube type undergoing the electronic transition e.g. $\mathrm{E}_{11}^{M}$ or $\mathrm{E}_{33}^{S}$. The importance of these sharp transitions will become apparent in Section 2.2.

The electronic properties discussed above are those of ideal, individual nanotubes with a diameter greater than $1 \mathrm{~nm}$. Outside this realm, perturbations that disturb the electronic structure occur; of which the most prominent is the curvature-induced effect for zig-zag tubes. For such tubes, finite curvature shifts the allowed wavevectors away from the K-point (Figure 2.3) giving rise to pseudogaps (red curve in Figure 2.4) of the order of $10 \mathrm{meV}$ (for $\mathrm{d}=0.4 \mathrm{~nm}$ ) [26]. Though this effect is not restricted to small diameter zig-zag nanotubes, the bandgap de- 
creases quickly with larger diameter $\left(\sim 1 / d^{2}\right.$ dependence) [26].

While armchair nanotubes are not greatly affected by finite curvature, they are also susceptible to electronic perturbation. Inter-tube interactions break their intrinsic symmetry and introduce a pseudogap around $200 \mathrm{meV}$ [27] at the Fermi level. The existence of such pseudogaps was theoretically predicted by Delaney et al. [28] and subsequently verified by scanning tunnelling microscopy (STM) $[27]$.

The opening of a gap at the Fermi level is a characteristic of metallic tubes due to the non-zero DOS. But the two effects discussed above also affect chiral nanotubes. The observed electronic characteristics stray from predicted zone folding calculations due to finite curvature and require other techniques such as the tight binding approximation (TBA) to match theory with experiment. Similarly, inter-tube interactions cause broadening and downshifting of the $\mathrm{E}_{i i}$ electronic transitions. [29, 30]. These effects will be covered in greater detail in Section 2.2.3.

The electronic nature of the constituent nanotubes within a bulk sample can greatly alter the macroscopic electrical properties where enriched samples of a particular electronic type will dominate conduction. For example, films where the conduction paths incorporate many semiconducting tubes show non-linear behaviour as a function of applied field [12] and other characteristics that correspond to macroscopic semiconductors. On the other hand, in thick mats where the conduction paths are predominantly metallic, the conductivity shows metallic characteristics [31]. The pressing point is that such an array of electronic properties are only possible due to the unique structure of SWNTs where simple variations in the $(n, m)$ index can dramatically alter the electronic characteristics. 


\section{$2.2 \quad$ Raman spectroscopy}

The Raman effect was first isolated and studied by the Indian physicist C.V. Raman $[32,33]$. The benefit of this technique lies in the sheer wealth of molecular information that can be extracted by using monochromatic light. Today, the Raman effect is a widely-used tool used for classifying and analysing chemical compounds.

When photons from a monochromatic beam of light hit a sample, the majority undergo elastic scattering, also known as Rayleigh scattering. A small fraction of these photons experience inelastic collisions with phonons. Phonons are quanta of vibrations whose vibrational frequency is intricately dependent on the symmetry and constituent atoms of the molecule.

The Raman effect is the result of an inelastic interaction between a photon and a phonon. When the photon loses energy via an inelastic collision to a phonon mode, the resultant photon leaves with $E_{\text {final }}=E_{\text {photon }}-E_{\text {phonon }}$. This precise loss of energy results in sharp spectral features compared to spectroscopic techniques like UV-Vis where photon interactions are generally broad and featureless. The Raman shift is usually measured in wavenumbers $\left(\mathrm{cm}^{-1}\right)$ and is taken as the difference between the incident and scattered photon frequencies $\left(1 / \lambda_{\text {inc }}\right.$ $1 / \lambda_{\text {scatt }}$ ) where $\lambda$ is the photon wavelength in $\mathrm{cm}$. The position and intensity of the peaks displayed on the spectrum can be used as a molecular signature.

The selection criterion for Raman scattering is a non-zero change in polarisability with vibrational motion. The polarisation $(\mathbf{P})$ of a molecule as a function of electric field ( $\mathbf{E})$ is given as: $\mathbf{P}=\alpha \mathbf{E}$ where $\alpha$ is the polarisability [34]. Certain molecular vibrations such as the one shown in Figure 2.5 change the polarisability so that $\mathbf{P}$ is now given as: 


$$
\mathbf{P}=\alpha \mathbf{E}+\left(\frac{\delta \alpha}{\delta Q} Q\right) \mathbf{E}
$$

where $\mathrm{Q}$ is the co-ordinate of a molecular vibration. If we simplify Equation 2.5 to one dimension using $\mathrm{E}_{x}=E_{x}^{0} \cos \left(2 \pi \nu_{0}\right) t$ and $\mathrm{Q}_{x}=Q_{x}^{0} \cos \left(2 \pi \nu_{i}\right) t$ where $\nu_{0}$ and $\nu_{i}$ are the photon and phonon frequencies respectively, we can rewrite Equation 2.5 as:

$$
\mathbf{P}=\alpha E_{x}=E_{x}^{0} \cos \left(2 \pi \nu_{0}\right) t+\left(\frac{\delta \alpha}{\delta Q} Q\right) E_{x}^{0} \cos \left(2 \pi \nu_{0}\right) t Q_{x}^{0} \cos \left(2 \pi \nu_{i}\right) t
$$

Equation 2.6 can be further simplified using the trigonometric identity: $\cos \theta_{1} \cos \theta_{2}=$ $\frac{1}{2}\left(\cos \left(\theta_{1}-\theta_{2}\right)+\cos \left(\theta_{1}+\theta_{2}\right)\right)$.

$$
\begin{aligned}
\mathbf{P}= & E_{x}^{0} \cos \left(2 \pi \nu_{0}\right) t \\
& +\frac{1}{2}\left(\frac{\delta \alpha}{\delta Q} Q\right) E_{x}^{0} Q_{x}^{0} \cos \left(2 \pi\left(\nu_{0}-\nu_{i}\right) t\right) \\
& +\frac{1}{2}\left(\frac{\delta \alpha}{\delta Q} Q\right) E_{x}^{0} Q_{x}^{0} \cos \left(2 \pi\left(\nu_{0}+\nu_{i}\right) t\right)
\end{aligned}
$$

Equation 2.7 describes elastic scattering where the photon survives a collision with a molecule without any change in energy. The remaining two terms depict the two photon-phonon interactions of the Raman effect. The first is the common Stokes effect where the photon loses energy to a phonon (Equation 2.8). The second is the inverse effect where the photon gains energy from a phonon and is known as the Anti-Stokes effect (Equation 2.9). Most molecular signatures only rely on the Stokes effect however, more sophisticated molecular analyses use both these effects.

Inelastic collisions are rare and the Raman signal is very weak - one of the 


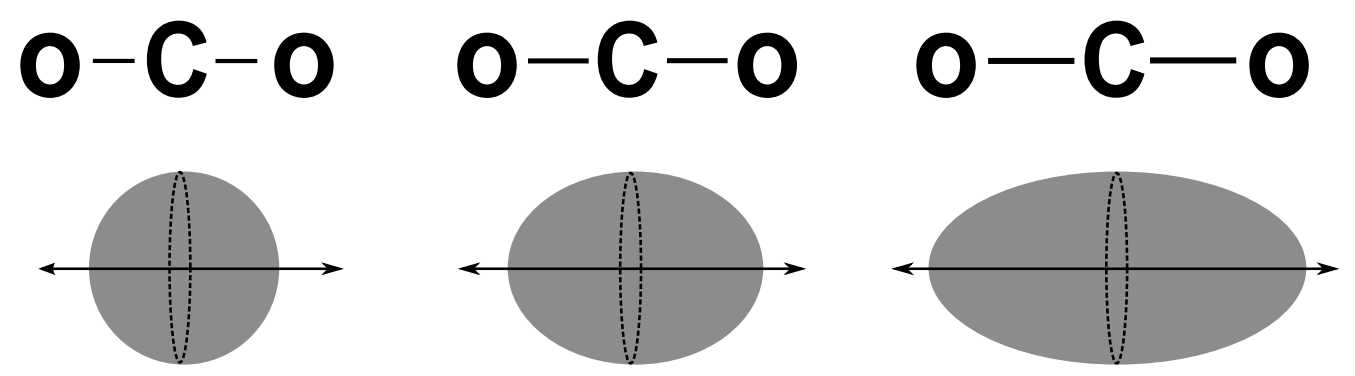

Figure 2.5: The symmetrical stretching mode of $\mathrm{C}_{2}$ is Raman active because the polarisability (depicted in grey) changes with the vibration

reasons it lay undiscovered until the 1930s. The most widely used method for signal amplification is the tuning of the laser energy to match an electronic transition. This results in a signal several orders of magnitude greater $\left(10^{2}-10^{4}\right)$ which can be reliably isolated by simple optics to give clean spectra with high signal-to-noise ratios.

Molecules with delocalised $\pi$ orbitals that can be easily distorted usually display strong Raman signals. $\mathrm{Sp}^{2}$ hybridised $\mathrm{C}-\mathrm{C}$ bonds of SWNTs have a delocalised $\pi$-electron cloud that can be perturbed by molecular vibrations. The strong Raman signature of SWNTs is further amplified by the resonant Raman effect. As discussed in the previous section, the 1D DOS of SWNTs contains Van Hove singularities with energy spacing proportional to the nanotube diameter. The spikes in the electronic density of states are clearly-defined electronic transitions $\left(\mathrm{E}_{i i}\right)$ that can be matched with lasers in the UV-NIR range.

The spectral features of a SWNT Raman spectrum are distinct and each of the discrete modes paint a clear picture of a statistically significant portion of the SWNTs under the laser spot. Figure 2.6 shows the four characteristic spectral features for SWNTs: radial breathing modes, G-mode, D-mode and G'-mode. Each of these modes will be discussed in detail below. 


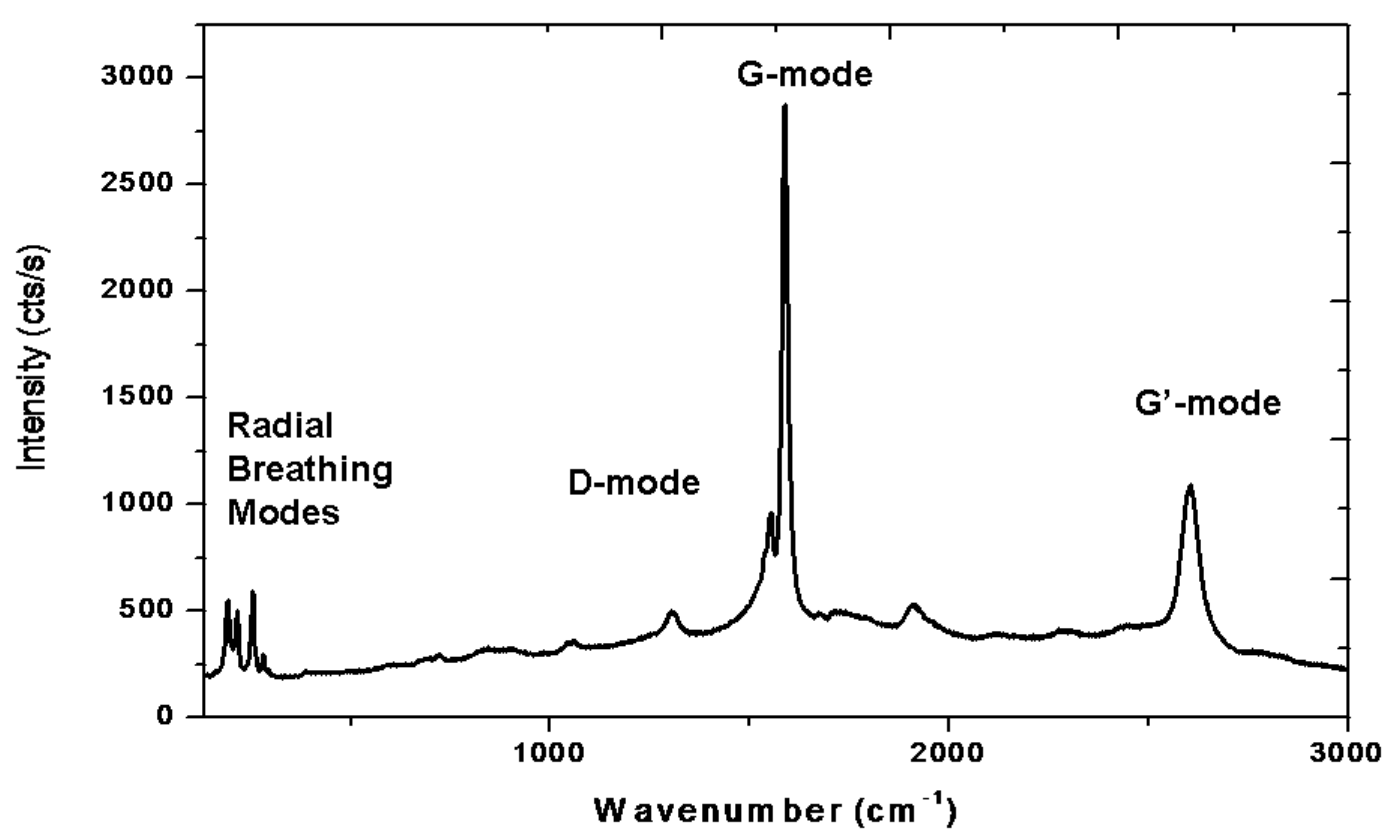

Figure 2.6: The Raman spectrum for an ensemble of SWNTs prepared in this study.

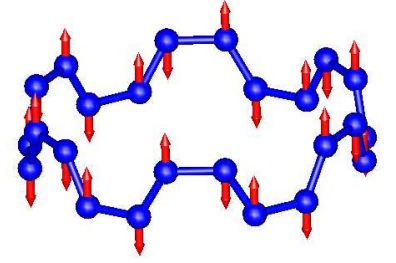

LO

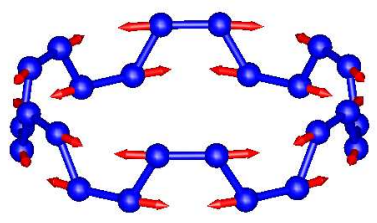

TO

Figure 2.7: The LO and TO vibrational modes of the G-band [35].

\subsubsection{The G-mode}

The graphite-mode or G-mode in graphite is a doubly degenerate vibrational mode of $\mathrm{E}_{2 g}$ symmetry at the $\Gamma$ point (where the wavenumber is zero) of the phonon dispersion. The G-mode is the legacy of C-C in-plane vibrational modes of graphite. Zone folding of the graphite phonon dispersion breaks the $\mathrm{E}_{2 g}$ symmetry into a combination of vibrational modes that contribute the most to the Raman signal. Though two main peaks $\left(\mathrm{G}^{+}\right.$and $\left.\mathrm{G}^{-}\right)$can be resolved by the naked eye, group theory predicts that the G-mode can be deconvoluted with six 


\begin{tabular}{|c|c|}
\hline Wavenumber $\left.\mathbf{( c m}^{-1}\right)$ & Symmetry \\
\hline 1552 & $\mathrm{E}_{2 g}$ \\
\hline 1569 & $\mathrm{~A}_{1 g}+\mathrm{E}_{1 g}$ \\
\hline 1592 & $\mathrm{~A}_{1 g}+\mathrm{E}_{1 g}$ \\
\hline 1607 & $\mathrm{E}_{2 g}$ \\
\hline
\end{tabular}

Table 2.1: Experimentally observed values of the four G-band modes for semiconducting SWNTs in a bundled sample [36].

Lorentzian oscillators for chiral tubes that are a mixed set of $\mathrm{A}_{1 g}, \mathrm{E}_{1 g}$ and $\mathrm{E}_{2 g}$ symmetries [36].

The longitudinal optical (LO) and tranverse optical (TO) branches of optical phonons (Figure 2.7) are mixed symmetry modes that give rise to the $\mathrm{G}^{+}$and $\mathrm{G}^{-}$peaks respectively for semiconducting tubes (see Table 2.1). Experimentally, the G-mode of an isolated semiconducting tube can be fit with two Lorentzian oscillators [37]. The frequency of the $\mathrm{G}^{-}$peak is dependent on the chirality of the nanotube. In a bundled sample containing mainly semiconducting tubes of mixed chirality, the G-mode can be fit with four (five in some cases) Lorentzian oscillators. The peak positions are given in Table 2.1. Figure 2.9 shows that the $\mathrm{G}^{-}$peak has a weak diameter dependence. The vibrational frequency is softened in narrow tubes due to enhanced phonon coupling with the electron continuum (i.e. $\pi$ electrons) [37].

In metallic tubes, the effect of curvature is superseded by phonon coupling to the electronic continuum [39]. Only the $\mathrm{G}^{-}$and $\mathrm{G}^{+}$peaks are visible in the G-mode. The lineshape of the $\mathrm{G}^{-}$peak is significantly broadened to a BreitWigner-Fano (BWF) lineshape (Figure 2.8): an intrinsic feature of individual metallic tubes [40]. A similar though more dramatic decrease in wavenumber with decreasing diameter is observed for metallic tubes due to an enhanced coupling with the narrower SWNTs (Figure 2.9) [37]. 


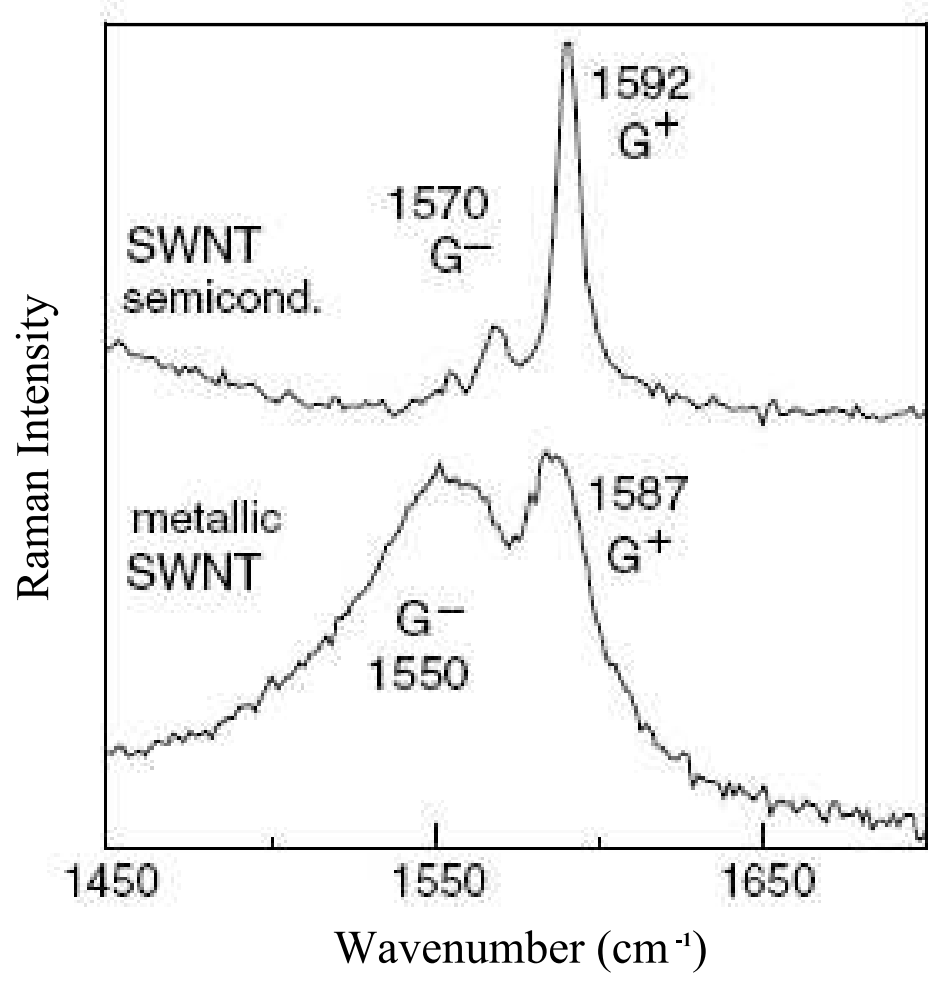

Figure 2.8: G-mode lineshape for metallic and semiconducting tubes. Modified from [38].

\subsubsection{Double resonance peaks: The D-mode and G'-mode}

The strongest peaks in the Raman spectrum like the G-mode are due to first order phonon scattering. However, there are many weaker peaks in the Raman spectrum that arise due to second order processes. Of these, the D-mode and the G'-mode are the most prominent.

Electronic resonance for the D-mode peak (at $\sim 1350 \mathrm{~cm}^{-1}$ with $\mathrm{E}_{\text {laser }}=2.41$ $\mathrm{eV}$ ) occurs near the K point (where the phonon wavenumber is $2 \pi / \mathrm{a}$ ) [41]. The excited electron does not immediately decay as a first order process. Instead, the electron emits a phonon with wavevector $\mathbf{q}$, causing it to traverse across the electron dispersion from $\mathrm{E}(\mathbf{k})$ to $\mathrm{E}(\mathbf{k}+\mathbf{q})$. The electron then decays back to the original K point by elastic scattering from defect sites [42]. Most defect sites in SWNTs have $\mathrm{sp}^{3}$ character. This change in hybridisation occurs at points of 


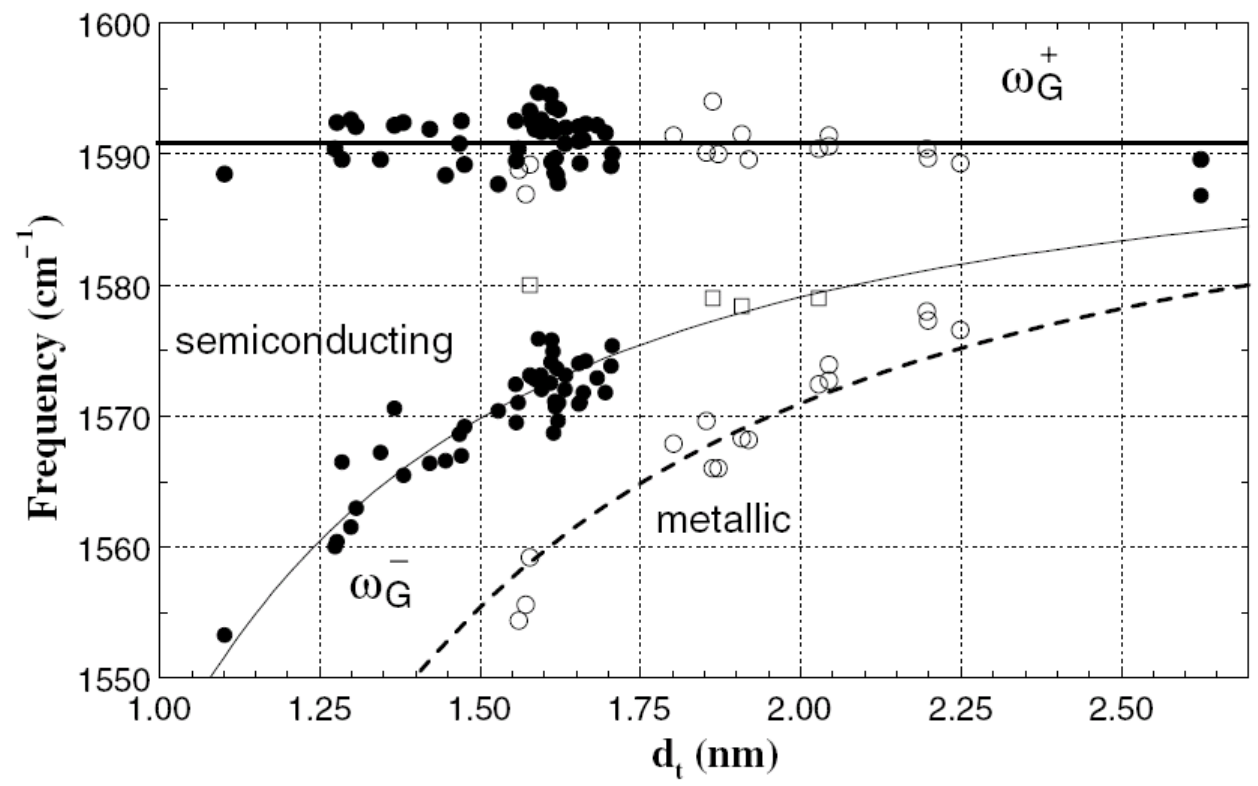

Figure 2.9: Graph showing the dependence of the $\mathrm{G}^{-}$peak on the diameter and electronic type. The filled black circles represent semiconducting tubes whilst the open circles represent metallic tubes [37].

physical stress (e.g. twists or bends in C-C network) or functionalisation. Hence, a greater fraction of these defects will correspondingly increase the D-mode intensity and can be used to monitor sample quality or the extent of chemical functionalisation.

The G'-mode at $\sim 2700 \mathrm{~cm}^{-1}$ is the second harmonic of the D-mode. In this case, the second order process involves two phonons of equal and opposite momenta. The first phonon is emitted when the electron moves from $\mathrm{E}(\mathbf{k})$ to $\mathrm{E}(\mathbf{k}$ $+\mathbf{q})$ and the second when the electron decays from $\mathrm{E}(\mathbf{k}+\mathbf{q})$ to $\mathrm{E}(\mathbf{k})$. Since the G'-mode is an intrinsic feature of the Raman spectra for graphitic materials, it is used as the reference for evaluating the relative intensity of the D-mode. 


\subsubsection{Radial breathing modes}

The radial breathing mode or RBM is the out-of-plane circumferential vibration of SWNTs. These modes have a vibrational frequency that is inversely proportional to nanotube diameter. Isolated SWNTs follow a simple empirical relation where $\omega_{R B M}=c_{1} / d_{T}$. Using this relation, a Kataura Plot [43] of laser energy and diameter can be created from tight-binding calculations or experimental studies of individual tubes. The proportionality constant, $c_{1}$, was theoretically evaluated to be $227 \mathrm{~cm}^{-1}$ though the experimental value was found to be $248 \mathrm{~cm}^{-1}$ [44].
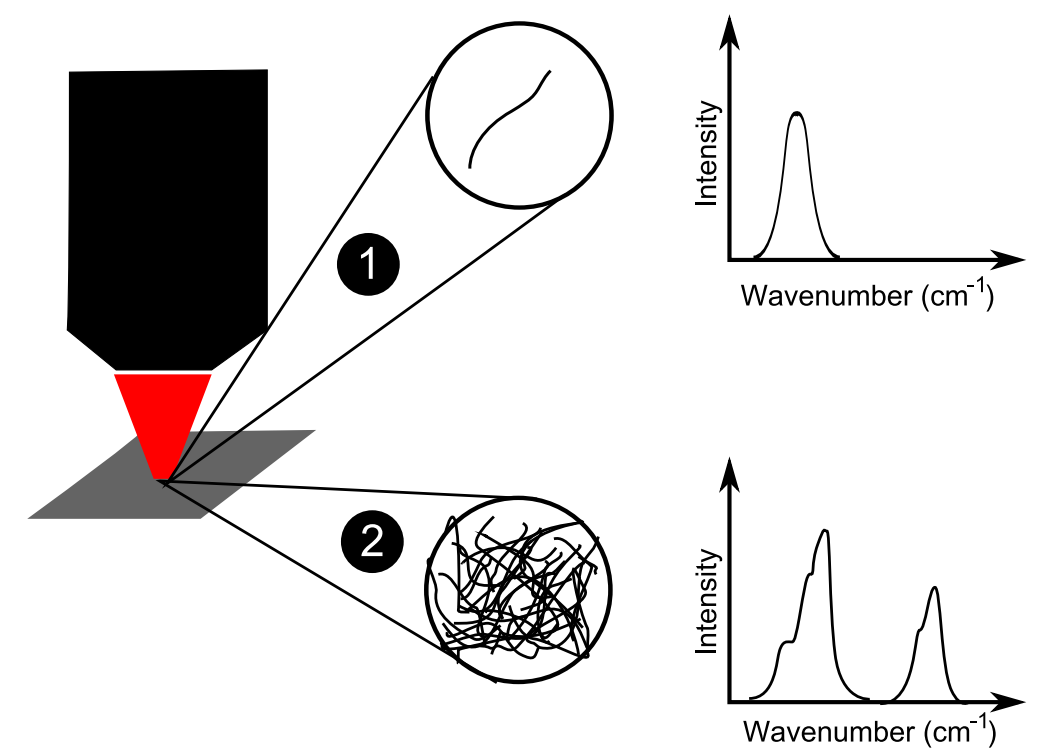

Figure 2.10: RBM spectra for isolated (1) and bundled (2) samples.

The study of individual tubes is essential for chiral index assignments and comparisons with theory. In the case of networks where SWNTs usually exist as ropes, an additional constant, $\mathrm{c}_{2}$ is required to account for inter-tube interactions giving $\omega_{R B M}=c_{1} / d_{T}+c_{2}$. The values for $c_{1}$ and $c_{2}$ change depending on the size and orientation of the bundles. A review of both theoretical and experimental values from literature can be found in [26].

Experimental Raman studies on isolated nanotubes confirm that only one 
RBM is visible for any given nanotube (Figure 2.10 (1)). In dense samples, more RBMs are observed (Figure $2.10(2)$ ) since a greater number of nanotubes are present under the laser spot. The intensity and frequency of the RBMs observed in the spectrum represent a statistically significant portion of the resonant nanotubes in the samples. Hence, RBM parameters such as relative peak position and intensity are used to monitor the effects of chemical modification, separation procedures and purification. In the following sections, the physical insights possible from these parameters are discussed.

\section{RBM frequency}

The frequency of the Raman modes is dependent on the C-C bond. Hence, any change to the bond strength would result in a frequency shift for all the phonon vibrations. The covalent carbon bonds are affected by various physicochemical phenomena such as electron transfer, hydrostatic pressure and the nanotube environment.

Theoretical calculations by Henrard et al. predicted upshifts to $~ 10 \%$ in the RBM frequency of bundled nanotubes compared to isolated nanotubes [45] due to strong inter-tube interactions in the former. These predictions were confirmed by Milnera et al. who found that the calculated RBM frequencies were downshifted compared to experimental values [46]. They too concluded that the upshift in the experimental sample was due to van der Waals interactions between nanotubes.

Large downshifts in the RBM frequency (up to $10 \mathrm{~cm}^{-1}$ ) were observed by Rao et al. [47] for bundled samples. However, these shifts were termed as 'apparent shifts'. The authors reasoned that the shifted tubes were a different subset of nanotubes bought into resonance due to bundling (see next subsection of Sec- 


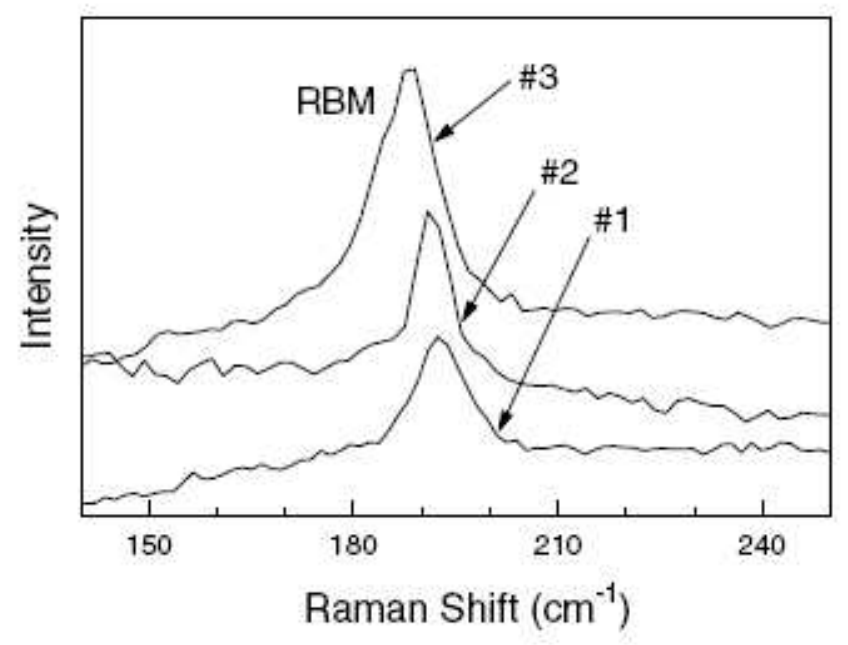

Figure 2.11: The change in RBM frequency as a function of bundle size. \#1 is the RBM spectrum of an individual tube. \#2 and \#3 are the spectra obtained from bundles $1.5 \mathrm{~nm}$ and $5 \mathrm{~nm}$ thick respectively. [48].

tion 2.2.3).

Figure 2.11 shows RBM spectra obtained from an isolated nanotube and two bundles of different sizes by Duesberg et al. [48]. According to Figure 2.11, the RBM frequency decreases from $\sim 195 \mathrm{~cm}^{-1}$ to $\sim 185 \mathrm{~cm}^{-1}$ as bundle size decreases. However, this spectrum only presents an 'apparent' shift similar to that of Rao et al. [47] rather than a real shift in the RBM frequency. To explain this effect, we refer to the experimental Kataura plot prepared by Mautlzsch et al. [49]. The Kataura plot shown in Figure 2.12 is not the traditional plot where wavenumbers are converted to diameter. Instead, it relates the wavenumber to the $\mathrm{E}_{i i}$ transitions which is convenient for comparisons to experimental $\mathrm{RBM}$ spectra. The red line in Figure 2.12 shows the He-Ne laser excitation at 632.8 nm: the same excitation used by Duesberg et al. to obtain the spectra shown in Figure 2.11. We can see that this laser excites many nanotubes between 180 - $200 \mathrm{~cm}^{-1}$ (indicated by the black box in Figure 2.12). Without another means of investigating the different chiralities present in the sample, it is not possible to unambiguously identify the RBMs. Hence, any shifts seen are 'apparent' only 


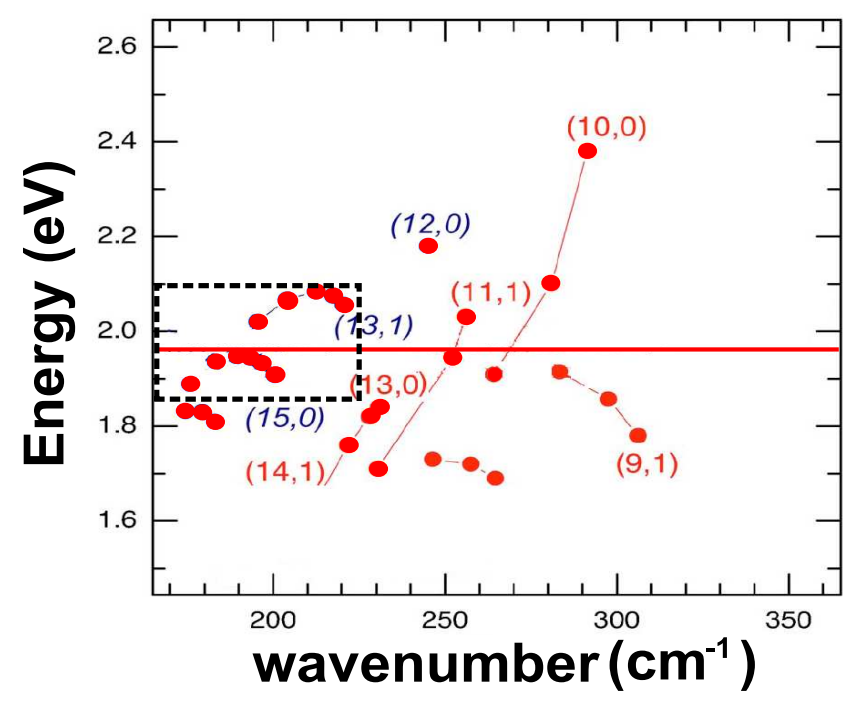

Figure 2.12: A Kataura plot of $\mathrm{E}_{i i}$ transition vs. wavenumber. Modified from [49]

since it is very likely that a different nanotube comes into resonance.

A study by O'Connell et al. also found no shifts in the RBM frequency between dispersed and bundled nanotubes though the null result was a consequence of poor spectrometer resolution $\left(4 \mathrm{~cm}^{-1}\right)$ [30]. The only positive finding of bundling-induced RBM shifts was reported by Murakami et al. [50]. Upshifts of $\sim 3 \mathrm{~cm}^{-1}$ in the RBM frequency were observed in centrifuged dispersions compared to bundled samples. The reasons for the shift were postulated to be due to the narrow $\mathrm{E}_{i i}$ transitions in dispersed nanotubes (see next subsection of Section 2.2.3). However, shifted and narrowed $\mathrm{E}_{i i}$ transitions would only cause a change in the population of resonant nanotubes not the frequency of the vibrational modes. Phonon vibrations are dependent on the vibrational force constant which in turn depends on the strength of the C-C bond. Only a change in the bond strength would result in a frequency shift. These changes can be induced physically by pressure $[51,52,53]$ or chemically via charge transfer $[49,54,55]$.

While investigating the RBM shifts due to unbundling, Izard et al. [51] found 
that the mere presence of surfactant was sufficient to cause a frequency shift. They claimed that the RBM upshifts in dispersions were due to the pressure exerted by the surfactant on the nanotube sidewall. By equating the cohesive energy density (CED) - the energy quantifying the van der Waals interaction in a liquid - to surfactant pressure [56], they found their results to be in agreement with shifts induced by a hydrostatic pressure of several hundred MPa [52, 53]. Izard et al. summarised the findings from Venkateswaran et al. [53] and Sandler et al. [52] on the effect of hydrostatic pressure on the Raman shift. The linear relation with pressure was summarised as: $7-10 \mathrm{~cm}^{-1} \mathrm{GPa}^{-1}$.

On the other hand, Maultzsch et al. proposed that a charge transfer mechanism was responsible for any observed shifts in the RBMs [49]. Nanotubes donating charge to the surfactant showed an upshift in RBM frequency due to stiffening of the C-C bonds. Downshifts in frequency were observed upon charge donation from the surfactant. The charge transfer theory is supported by evidence of Raman shifts in doped nanotubes $[54,55]$.

RBM frequency shifts arise due to changes in the bundle size, pressure from surfactant or charge transfer. Of these, the charge transfer mechanism is the most widely studied. The remaining mechanisms are relatively unstudied outside the literature mentioned above. Section 5.1.3 considers these mechanisms as a possible explanation for RBM shifts observed between the nanotube dispersion and raw powder.

\section{RBM intensity}

The RBMs represent SWNTs in the sample with electronic transitions that are in resonance with the laser energy. Assuming that there is no bias in the electronphonon coupling, the intensity could be considered a measure of the relative 
population of resonant nanotubes. This information could in turn lead to an estimation of enrichment by electronic type, chirality or diameter. However, this method fails on two accounts. The first is the assumption of constant phonon coupling though this effect can be accounted for if comparisons are made between two spectra. The second failing is the change in intensity of a given RBM depending on the nanotube environment. The electronic transitions of a nanotube in a bundle change considerably depending on the size of the bundle.

The resonance window for individual tubes in solution is $\sim 40 \mathrm{meV}$ [57]. Thus, for a $632.8 \mathrm{~nm}(1.96 \mathrm{eV})$ laser, SWNTs with $\mathrm{E}_{i i}$ between $1.92 \mathrm{eV}$ and $2.0 \mathrm{eV}$ are resonantly excited and visible in the Raman spectrum. Inter-tube coupling broadens the resonance window [29, 30] as much as $200 \mathrm{meV}$ [58] and downshifts the $\mathrm{E}_{i i}$ transition energies $[29,30]$. These changes to the $\mathrm{E}_{i i}$ transitions are depicted in Figure 2.13.

The introduction of an orthogonal electronic dispersion is implicated as the cause for broadening $\mathrm{E}_{i i}$ [59]. The electronic dispersion for $1 \mathrm{D}$ nanomaterials such as SWNTs is along the nanotube axis. However, this dispersion develops an additional dimensionality in bundles where many tubes are aligned next to each other. Due to broadening, the resonance condition is significantly altered to include tubes with $\mathrm{E}_{i i}$ from $1.72 \mathrm{eV}-2.2 \mathrm{eV}$ leading to more tubes visible in the RBM spectrum. The intensity of RBM peaks that arise due to bundling (sometimes referred to as 'bundling' peaks) are often used as a qualitative measure of aggregation [60].

The downshifting of $\mathrm{E}_{i i}$ alters the relative intensities of the resonant nanotubes. Since RBM signal intensity depends on the match between the relevant $\mathrm{E}_{i i}$ and the laser energy; changes to the $\mathrm{E}_{i i}$ move the nanotube concerned from 


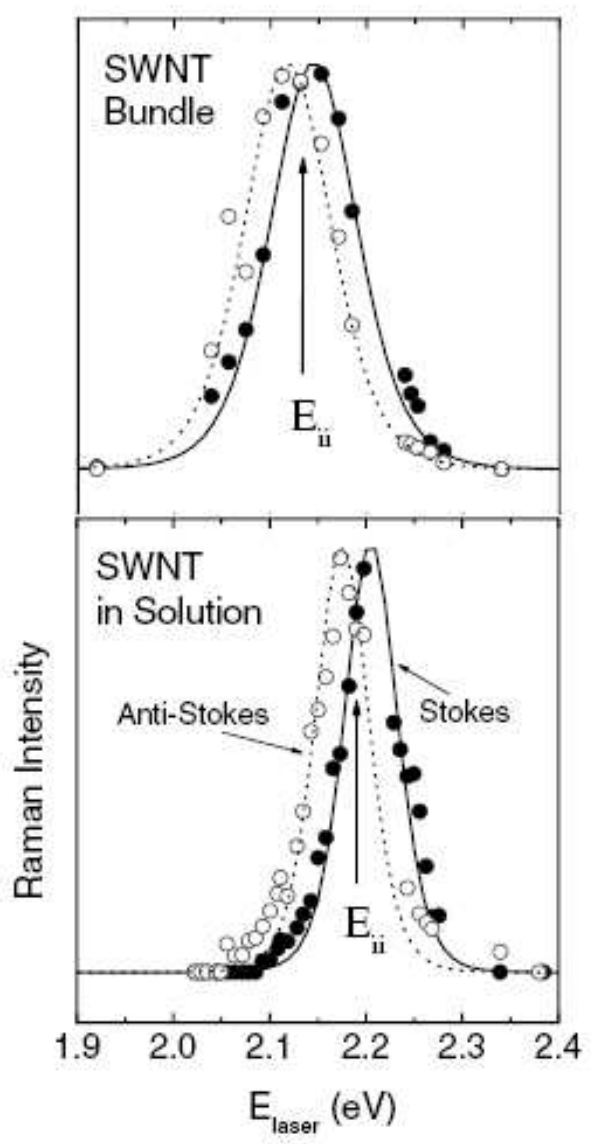

Figure 2.13: The downshifting and broadening of the $\mathrm{E}_{11}$ electronic transition for a tube within a bundle due to inter-tube coupling [29].

the excitation energy and result in a different relative intensity. The schematic in Figure 2.14 clearly shows the introduction of new peaks upon bundling (b) and the changes in relative intensity as bundling becomes pronounced (c) - (d).

Laser-induced oxidation and annealing can also change the relative intensities of the RBM and D-mode respectively [60]. Small diameter nanotubes combust even under a relatively low power density of $\sim 1 \mathrm{~W} / \mu \mathrm{m}^{2}$, thus skewing the distribution of the nanotube ensemble.

In conclusion, the effects due to bundling and laser oxidation need to be carefully considered before embarking on Raman studies to quantify the effects of external factors. While the consequences of bundling can complicate RBM 

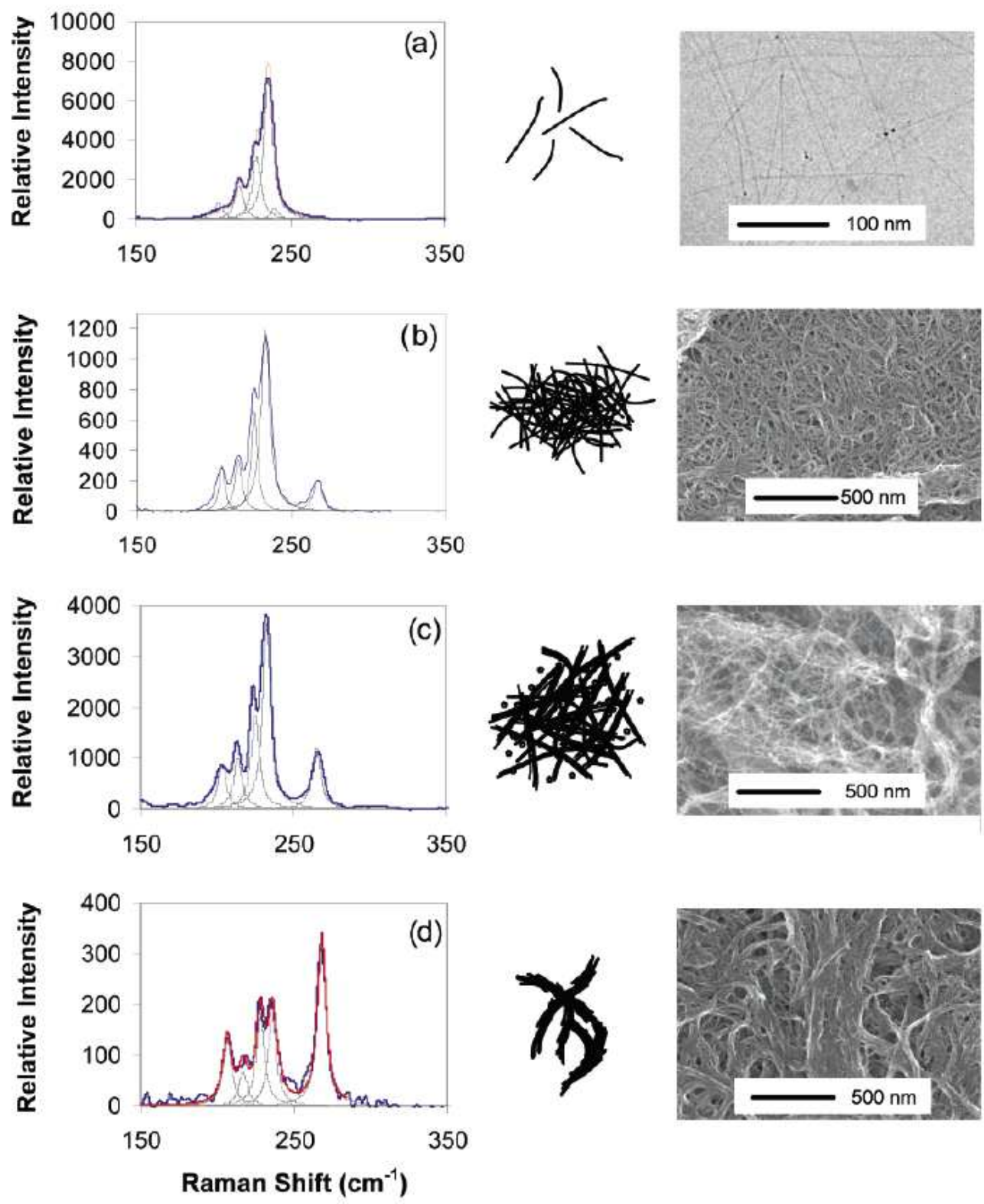

Figure 2.14: The effects of morphology on the observed RBM frequencies and relative intensities [57].

analysis, it can also be used as a tool to qualitatively understand the extent of aggregation within the sample. Section 5.1.2 uses the concept of the resonance window to probe the extent of bundling within an aggregated sample and an exfoliated nanotube dispersion. 


\subsection{Conduction in SWNT networks}

Connections between nanotubes in the network can be studied by percolation theory $[61,62]$. A simple analogy of pick up sticks can be employed to understand the basis of this theory. This stick model is sufficient to describe conduction in SWNT networks regardless of chirality since more than $80 \%$ of the network conductance is due to connected metallic nanotubes [63]. A small pile of scattered sticks in a unit square form a sparsely connected network with few connected pathways. Inter-connectivity can be increased by adding more sticks to the pile: Figure 2.15 (c) compared to Figure 2.15 (a). The change in conductance $(\mathrm{G})$ of the network as a function of concentration is:

$$
G=\left(N-N_{c}\right)^{\alpha}
$$

The percolation threshold described by $\mathrm{N}_{c}$ is the weight fraction at which the thin film begins to conduct. Once this threshold is attained, the conductance rises superlinearly with the critical exponent $(\alpha)$. Equation 2.15 is only valid near $\mathrm{N}_{C}$ since the conductance reaches saturation at some point away from the percolation threshold. The value of the critical exponent calculated using the simplistic model above changes with the dimensionality. Percolation for nanowire networks is mainly two or three dimensional ( $2 \mathrm{D}$ or $3 \mathrm{D})$. $2 \mathrm{D}$ percolation occurs in a percolating network that has a thickness of one monolayer whilst 3D percolation involves percolating pathways that traverse the network in depth. The value of

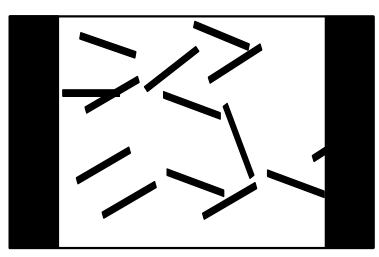

(a)

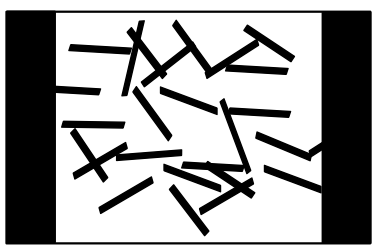

(b)

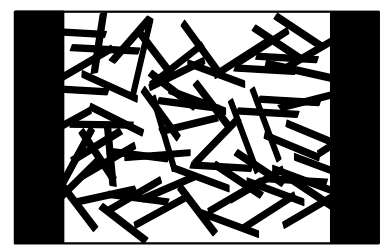

(c)

Figure 2.15: The pickup stick model for nanotubes. 


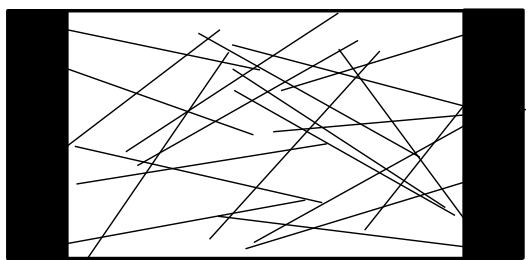

(a)

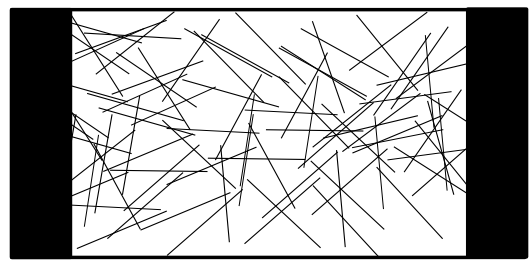

(b)

Figure 2.16: Longer tubes reduce overall junction resistance as inter-tube connections are fewer.

the critical exponent is 1.33 and 1.94 for $2 \mathrm{D}$ and 3D percolation respectively [64]. Ideally, the variation in $\alpha$ between 1.33 and 1.94 could be used as a gauge for film thickness but the lack of a consistent standard in current literature limits any insight.

Improvements to sheet conductance have been attempted by altering geometric parameters such as tube length and diameter [65, 66] since longer tubes reduce the number of inter-tube connections (Figure 2.16) required for percolation. However, the quality of the SWNTs is crucial for long tubes to have superior conducting properties. Structural defects introduced via sonication [67] and chemical treatment [68] cause electrons to scatter from defects which in turn interrupts ballistic conduction within the nanotube.

Percolation theory gives a good analytical description of the dependence of conductance on the weight fraction of SWNTs and geometrical parameters like length. However, it does not provide a detailed explanation of electron transport in the network. The fundamental assumption that electrons hop effortlessly from one tube to the next is invalid in the case of SWNT networks because conductance is impeded at inter-tube junctions by an energy barrier [69]. 


\subsubsection{Conduction mechanisms}

The rough picture of conduction in nanotube networks has been described as conducting regions separated by insulating barriers [69]. Junction barriers vary from one connection to the next depending on the nature of the coupling between the SWNTs which range from metallic-semiconducting tube connects to residual surfactant. Models of percolation conduction in networks where an inter-tube coupling constant is included do not capture the random nature of these barriers since an intimately entangled network has a broad spatial and energetic distribution of energy barriers.

In the following sections, a qualitative description of the prominent theories for conduction in these systems such as fluctuation induced tunnelling (FIT) [70] and variable range hopping (VRH) [71] will be provided.

\subsubsection{Fluctuation induced tunnelling (FIT)}

The theory for FIT was developed by P. Sheng to describe electron transport in disordered systems where metallic islands are separated by small non-conducting barriers (see Figure 2.17 (a)) [70]. In this model, the barrier size is considered to be insignificant compared to the size of the conducting segments. Electrons tunnel through the barrier with assistance from voltage fluctuations (see Figure 2.17 (b)). These voltage fluctuations are caused by the build up and deficit of charge and effectively reduce the size of the barrier 'seen' by the electrons leading to an increase in the tunnelling probability.

This model incorporates three temperature-dependent electron tunnelling mechanisms. At very low temperatures (as $\mathrm{T} \rightarrow 0$ ) there are few phonons to thermally excite or assist electrons through barriers and electron transport occurs by quan- 


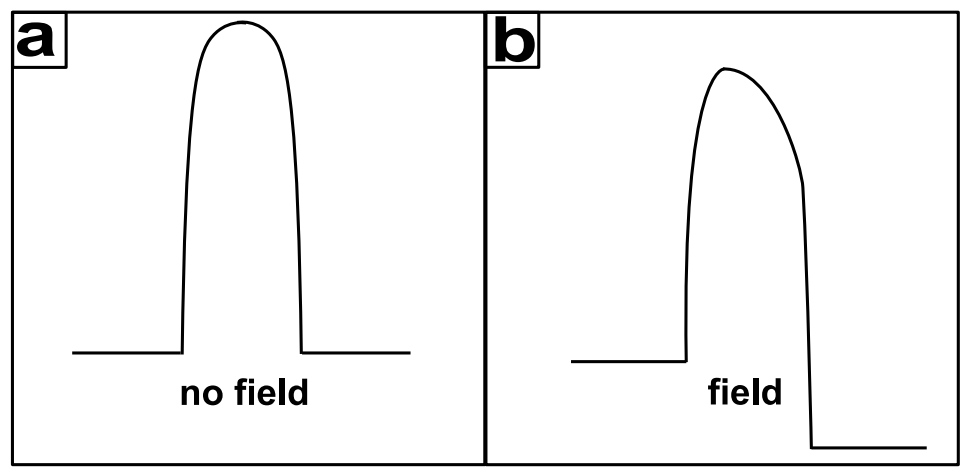

Figure 2.17: (a)The energy barrier between two conducting segments. (b) An electric field due to the voltage fluctuations increases electron tunnelling probability by reducing the barrier height and lowering the Fermi level on the other side. Modified from [70].

tum tunnelling. At high temperatures, thermal activation is mainly responsible for conduction whilst a combination of thermal activation and fluctuation induced tunnelling is predominant at intermediate temperatures. A closed form for FIT with a parabolic barrier (such as the one illustrated in Figure 2.17) is given as:

$$
G=G_{0} \exp \left(\frac{-T_{1}}{T+T_{0}}\right)
$$

where $T_{1}$ and $T_{0}$ are constants. $T_{0}$ is the temperature above which voltage fluctuations become important [70].

Equation (2.11) is widely used to fit temperature-dependent conductivity data in materials where the barriers between the conducting segments are thin. This model aptly describes conduction in thick mats and networks of SWNTs $[72,31]$. In these cases, the fraction of metallic nanotubes is large enough for the formation of highly conducting pathways with small barriers. As the network decreases in thickness, the number of connected metallic tubes also decreases correspondingly. Hence, barriers become larger and tunnelling probabilities dwindle to zero within the barrier. Inter-tube barriers also increase with impurity and defect concentration. In such cases, the FIT model becomes redundant and these datasets are 
treated with a mechanism known as variable range hopping.

\subsubsection{Variable range hopping}

VRH [71] is built on the foundation theory of Anderson localisation [73]. A rudimentary introduction to localisation is detailed below before proceeding to the intricacies of the hopping process.

\section{Localised states}

Band theory treats solids with a uniform lattice assuming a fundamental approximation that atoms are stationary ionic cores with delocalised electrons. The electrons can belong to a subset of stationary electronic states which they occupy according to Fermi-Dirac statistics. A periodic potential is used to simulate the ionic cores and the Schrodinger equation is solved for the Hamiltonian. The resultant wavefunctions take the form of a plane wave modulated by periodic Bloch functions.

Delocalised electrons are not bound in any way in a pure crystalline lattice. However, this delocalisation is interrupted when impurities are present. Impurities or lattice defects create bound states at the site. These bound or localised states have an exponentially decaying wavefunction away from the origin (Figure 2.18) where the decay factor $\alpha$ is the inverse of the localisation length $\left(\xi_{l o c}\right)$.

In semiconductors, localised states appear at band edges and within the electronic band gap [74]. A simple idea based on polychromatic percolation theory can be used to illustrate this [75]. A semiconductor material with some bandwidth (B) and a generic DOS distribution is shown in Figure 2.19. The DOS is 


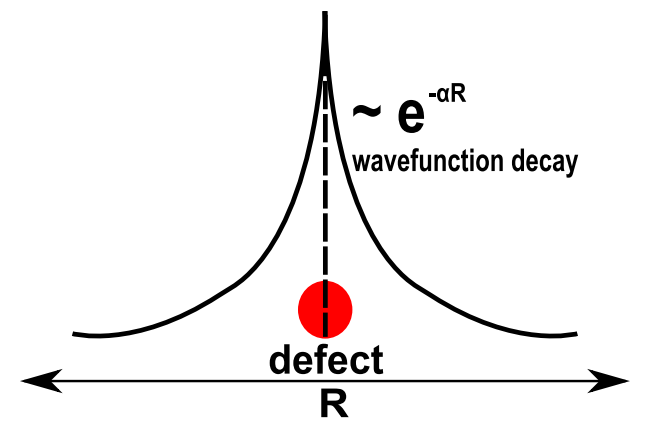

Figure 2.18: A lattice defect has a localised wavefunction that decays exponentially away from the defect site.

arbitrarily divided into $\mathrm{N}$ slices and each of the slices is assigned a colour. The constraint is that an electron state within a coloured slice can only move to a state within that slice. Near the DOS maximum, there are many available states for the electrons. Hence, they are delocalised.

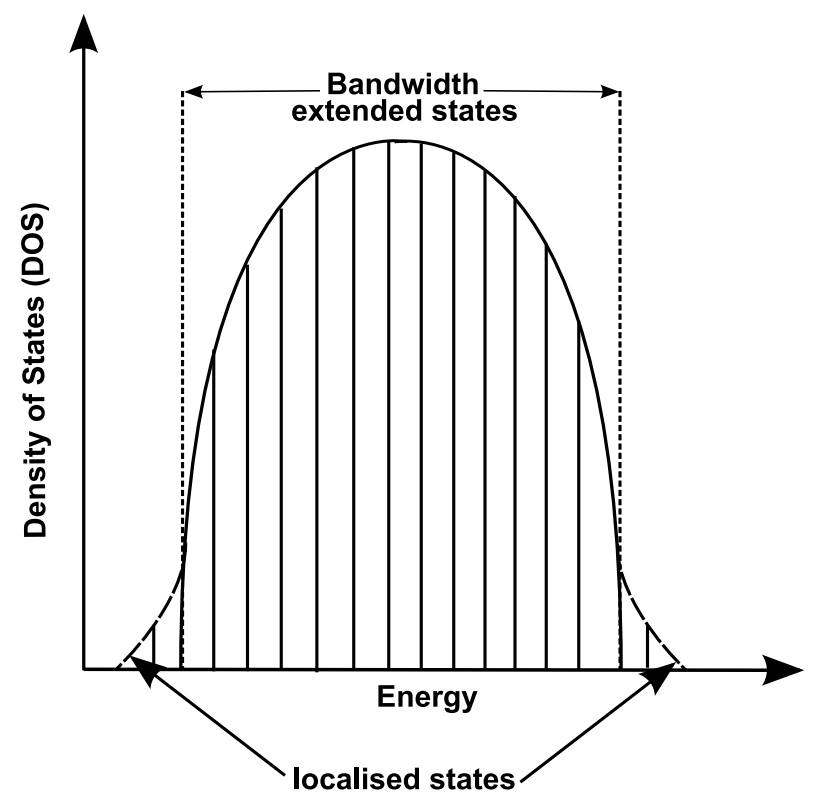

Figure 2.19: Density of States for a pure semiconductor (solid lines) and a partially disordered semiconductor (dashed lines).

Impurities on the other hand, are distributed randomly throughout the crystal. Their atomic potentials vary about some mean with a distribution $\mathrm{W}$ (see Figure 2.20). For such states, corresponding states of similar energy are few. As a result, localised states appear discretely within the bandgap. 

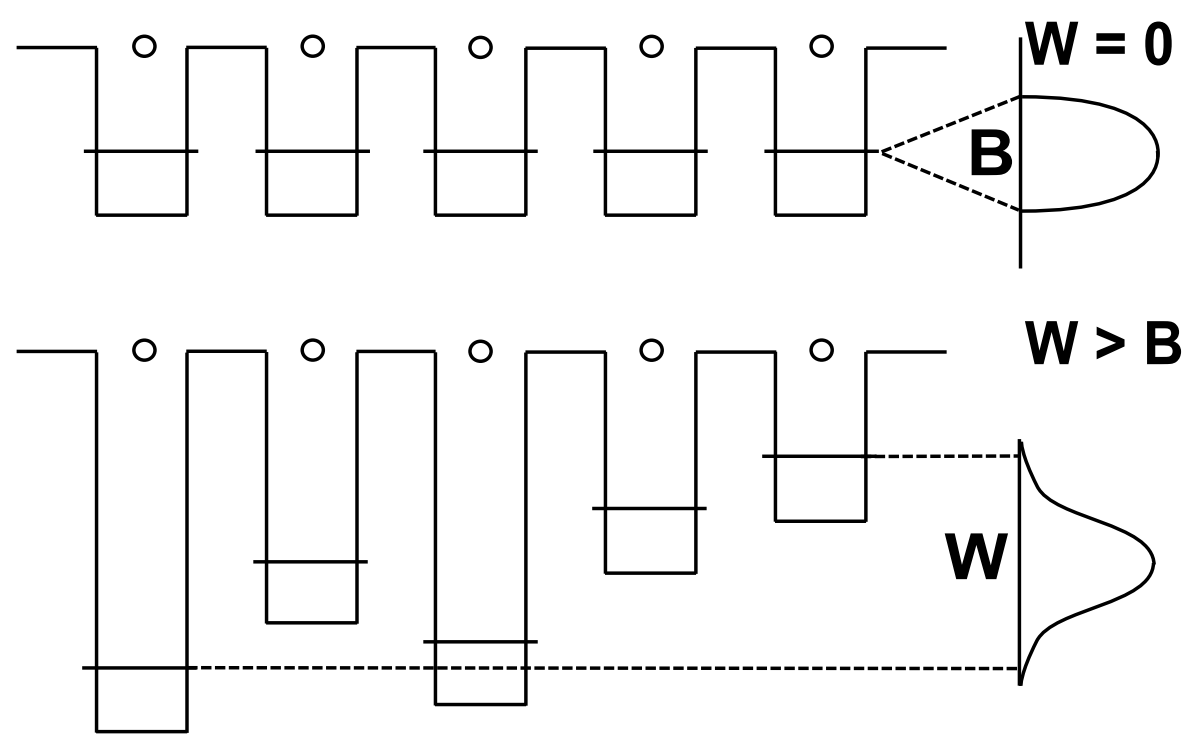

Figure 2.20: Crystalline lattice with $\mathrm{W}=0$, compared to an amorphous material where $\mathrm{W}>\mathrm{B}$. Adapted from Zallen [75].

The two limiting situations for a material are delocalised, extended states and completely localised states. If all ionic cores of a 1D lattice of atoms are the same i.e. a crystal, then the distribution of the fluctuating potential (W) is zero (Figure 2.20). The crystal bandwidth decreases considerably with increasing disorder while the fluctuating potential spreads the overall material bandwidth (Figure 2.20). And, at some critical fraction of disorder there is a transition from extended states to bound states. We can use perturbation theory to pinpoint when this transition occurs as a function of the parameters $\mathrm{W}$ and $\mathrm{B}$. The result of such a calculation has established the critical threshold when W exceeds B.

The pedagogical model of lakes and oceans [75] is a clear way to conceptualise the consequences of disorder. The situation corresponding to a fully disordered material with only localised states can be pictured as a collection of individual lakes. As disorder decreases, the transition from localisation to delocalisation takes place: some of the lakes form connections with each other creating an extended lake or ocean. Individual lakes continue to coexist with the greater 
ocean until extended states are restored and there are no more lakes left.

\section{Hopping Conductivity}

The theory of electron transport between localised states was derived by N.F Mott [71]. He proposed that electrons can hop between two localised states if their wavefunctions overlap. However, electrons are not only constrained in space but also in energy. From the picture of polychromatic transport discussed earlier, electrons can only hop to sites that have the same energy. In reality, the electrons can borrow thermal energy and marginally expand their list of final hopping sites.

Mott visualised an extended approach to polychromatic percolation where the travelling electron can also 'see' the spatial variations in energy of the different localised states within its Fermi window $\left(\mathrm{k}_{B} \mathrm{~T}\right)$. At high temperatures, the electron has sufficient thermal energy that a large number of sites are close enough in energy to accept it, and therefore there is a good chance that a suitable site is very close. At lower temperatures, the narrower Fermi window implies that the electron has to hop further to a site within its thermal energy.

Mathematically, the probability for hopping between any two sites can be written as a combination of spatial and energetic differences:

$$
P=\exp \left(-\Delta E_{i j} / k_{B} T\right) \exp \left(-\Delta R_{i j} / \xi_{l o c}\right)
$$

where $\Delta E_{i j}$ and $\Delta R_{i j}$ describe the energy and spatial differences respectively. In a 3D material, the number of states $(\mathrm{N})$ separated by the desired energy and distance can be written as a function of the density of states, $N(E)$ :

$$
N=\frac{4}{3} \pi \Delta R_{i j}^{3} N(E) \Delta E_{i j}
$$


For electron transport between any two sites, the number of states is simply equal to 1. Thus, Equation (2.12) can be re-written as a function of $\Delta R_{i j}$ with the condition from Equation (2.13):

$$
P=\exp \left(-\alpha / \Delta R_{i j}^{3} k_{B} T\right) \exp \left(-\Delta R_{i j} / \xi_{l o c}\right)
$$

where the constant $\alpha=3 /[4 \pi N(E)]$. Equation (2.14) can be maximised to give an optimum value for $\Delta R_{I j}$. This value can then be used as a constant and the conductance $(\propto \mathrm{P})$ is:

$$
G=G_{0} \exp \left(-T_{0} / T\right)^{\frac{1}{4}}
$$

Equation (2.15) is only in the 3D case but a generalised expression for some dimensionality $\mathrm{d}$, is be given as:

$$
G=G_{0} \exp \left(-T_{0} / T\right)^{\frac{1}{1+d}}
$$

where $\mathrm{G}_{0}$ is a geometrical prefactor and $\mathrm{T}_{0}$ can be related back to the localisation length using the relation:

$$
T_{0}=\frac{\lambda}{\xi_{l o c}^{d} N(E) k_{B}}
$$

where the constant $\lambda$ has been evaluated to be 16 by Ambegaokar et al. [76].

This approach for the deriving the Anderson criterion for localisation and the subsequent hopping mechanism is based on materials with constrained disorders. This class of materials is considerably different to nanowire networks of varying dimensionality. In SWNT networks, there is no inherent uniformity and the premise for localisation is based on the amount of local disorder where the $\mathrm{sp}^{2}$ nature of the carbon bonds are perturbed to $\mathrm{sp}^{3}$. This change to the VRH picture also changes the interpretation of the $\mathrm{G}_{0}$ parameter. In SWNT networks, it represents the number of conducting branches [77]. 
Conduction studies of SWNT networks have shown remarkably good fits with VRH $[31,72,77,78,79,80]$. Localised states arise due to remnant surfactant, defects at inter-tube junctions, irradiation sites or carbonaceous impurities [81]. Two main types of networks show dominant VRH conduction: networks with a high degree of impurities and very thin networks.

Networks with a large impurity concentration have a small localisation length. Transport via the abundant localised states dominates over FIT because the impurities plump up the barrier thickness. In thin networks, our earlier assumption of metallic conduction paths becomes invalid. Due to the dependence of the percolation threshold on the dimensionality, paths formed from metallic tubes may no longer dominate the conductivity. Instead, the paths are of mixed electronic type where a considerable number of junctions are metal-semiconductor junctions (also known as Schottky barriers). Given that these barrier energies exceed 200 meV [65, 82], hopping transport once again dominates over thermal activation and FIT in the intermediate to low temperature regimes. 


\section{Chapter 3}

\section{Characterisation techniques}

The schematic depicted in Figure 3.1 shows the sample preparation process. This chapter describes the various physical techniques used to characterise the samples at every step of the thin film fabrication process.

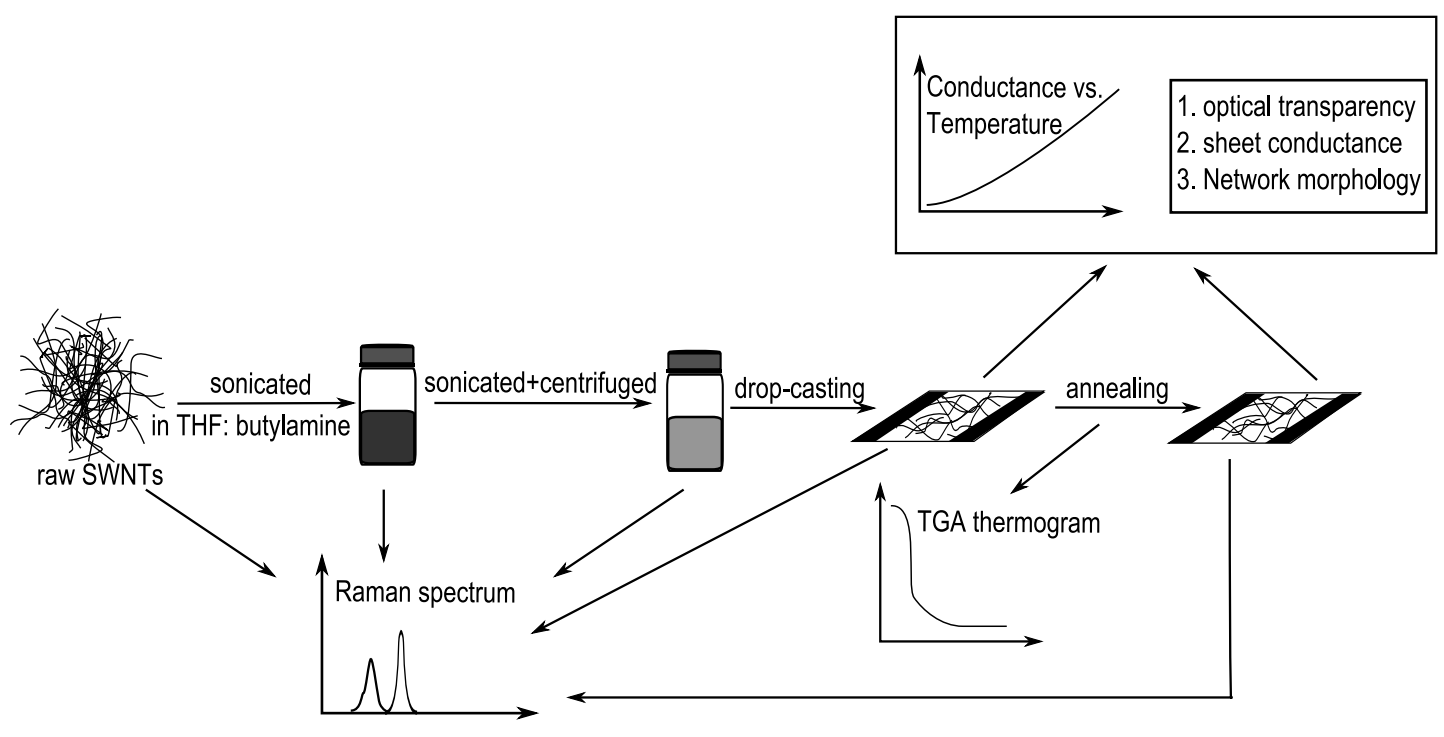

Figure 3.1: Schematic of the physical characterisation performed on SWNT dispersions and thin films. 


\subsection{Basic physical characterisation}

\subsubsection{Optical transparency}

An important aspect of SWNT thin film characterisation is the determination of film transparency. Optical transmission measurements were taken using a single beam HP 8452A diode array spectrometer for wavelengths from $200 \mathrm{~nm}$ to $820 \mathrm{~nm}$. The background - a blank coverslip - was manually subtracted before measuring film transmittance. Transmittance was measured across an area of $15 \mathrm{~mm}^{2}$ to average over local inhomogeneity. The relative transparency between different films was compared using the value at $550 \mathrm{~nm}$.

\subsubsection{Measuring conductance}

\section{Evaporating metal contacts}

Metal contacts for measuring sheet resistances were deposited by evaporating Nichrome $(\mathrm{Ni} / \mathrm{Cr})$ and gold contacts directly onto the substrates prior to film fabrication. Contacts with an electrode gap of $4 \mathrm{~mm}$ were evaporated using an Edwards E306A thermal evaporator as shown in Figure 3.2.

The thermal evaporator was renovated to include a 4-boat insert for bottomup evaporation (see Figure 3.3). Once the substrates were loaded, the chamber was pumped down to $6-8 \times 10^{-2}$ mbar first with a rotary vacuum pump and then to $6-8 \times 10^{-5}$ mbar with a water-cooled diffusion pump. The evaporation mask deposited $2 \mathrm{~mm}$ wide metal strips set $4 \mathrm{~mm}$ apart. Nichrome was evaporated from a boron nitride crucible using a maximum current of 52 A supplied to the basket. Once the Nichrome evaporation was complete, a thin film of gold was evaporated from a tungsten boat using a maximum current of $68 \mathrm{~A}$. 


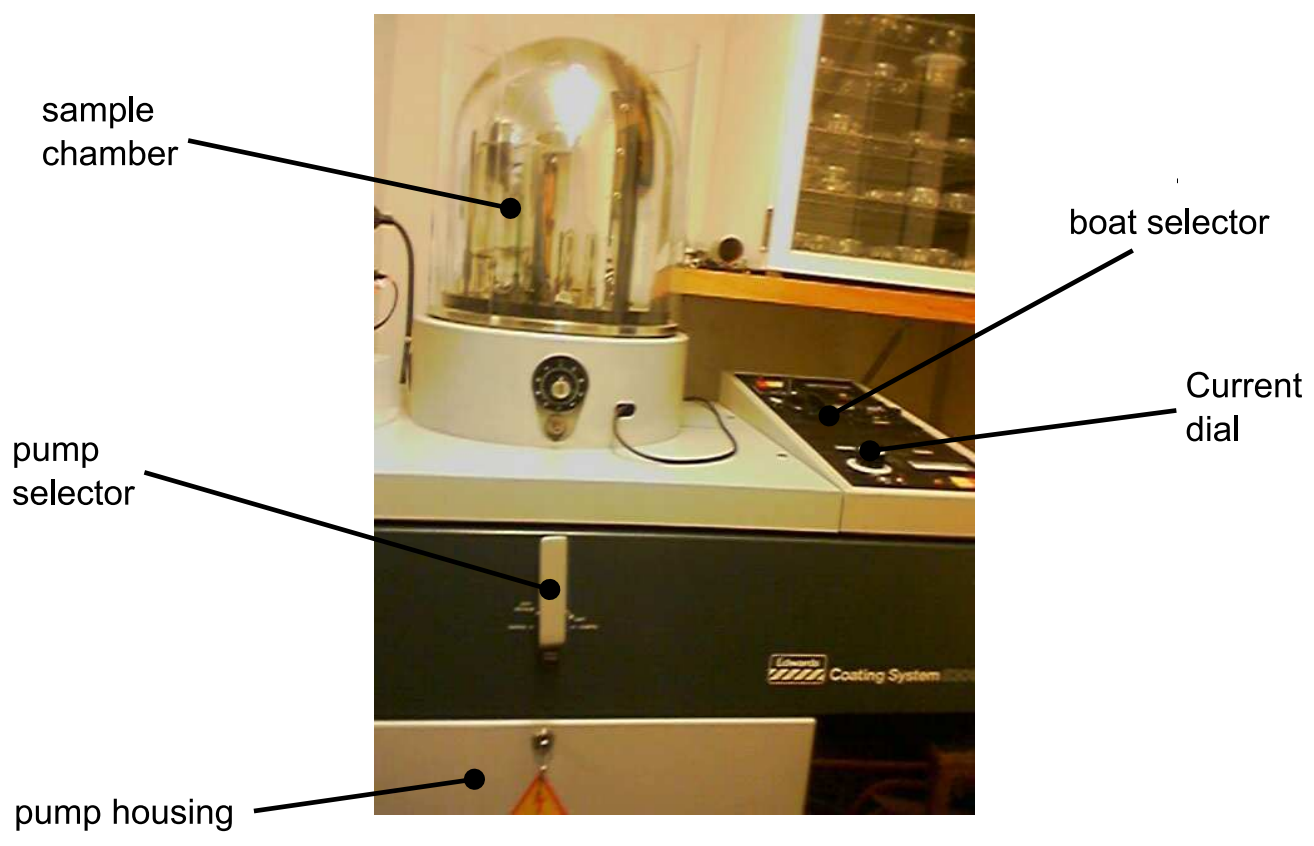

Figure 3.2: Edwards E306A thermal evaporator

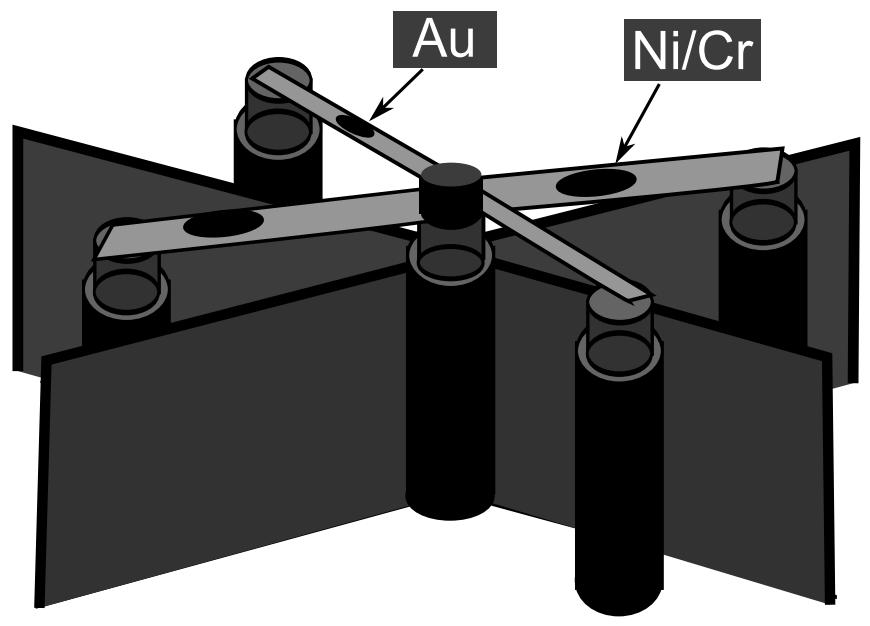

Figure 3.3: 4-boat insert for multiple metal evaporation.

\section{Measuring conductance}

All the values of sheet resistance and conductance reported in Chapters 4 were measured using a hand-held digital multimeter. To convert from the measured resistance to the conventional $\Omega / \square$, the measured value was multiplied by $2 / 3$. 


\subsubsection{Surface morphology}

\section{Scanning electron microscopy}

The scanning electron microscope (SEM) was used to image the surface morphology of SWNT thin films. In this technique, a high energy electron beam is rastered across the specimen and the resultant electrons from electron-sample collisions are used to construct a topographical image of the sample surface.

Electrons emitted from a hot tungsten tip are accelerated towards the specimen by a voltage bias of several thousand electron volts. En route to the sample, electromagnetic objectives and condensers are used to focus the electron beam to a tight spot. Magnification is controlled by rastering across smaller x,y dimensions on the sample. The incident beam interacts with the sample via inelastic and elastic collisions. Inelastic collisions result in the production of secondary electrons as well as X-rays. The strength of the secondary electron signal is intimately connected to the sample morphology. For example, a larger number of electrons escape from exposed edges giving an enhanced signal for these spots. These electrons are collected by a scintillation detector and subsequently amplified with a photomultiplier tube and an amplifier before being built into a digital image.

\section{Imaging conditions}

In this study, a JEOL JSM 6500F scanning electron microscope was used for imaging SWNT thin films. David Flynn (electron microscopy technician at VUW) performed all the instrumental calibrations and loading of the samples. Images were obtained with the secondary electron detector and an accelerating voltage of $6-8 \mathrm{keV}$. Magnifications ranging from $150 \times$ to $60,000 \times$ were possible without coating due to the high conductivity of the nanotubes. 


\subsection{Post-deposition treatment}

\subsubsection{Thermogravimetric analysis}

\section{Technical details}

Thermogravimetric analysis (TGA) is a technique that monitors the mass change of a sample as a function of temperature. The SDTQ600 DSC/TGA model manufactured by TA Instruments was used to obtain thermograms of butylaminecoated SWNTs.

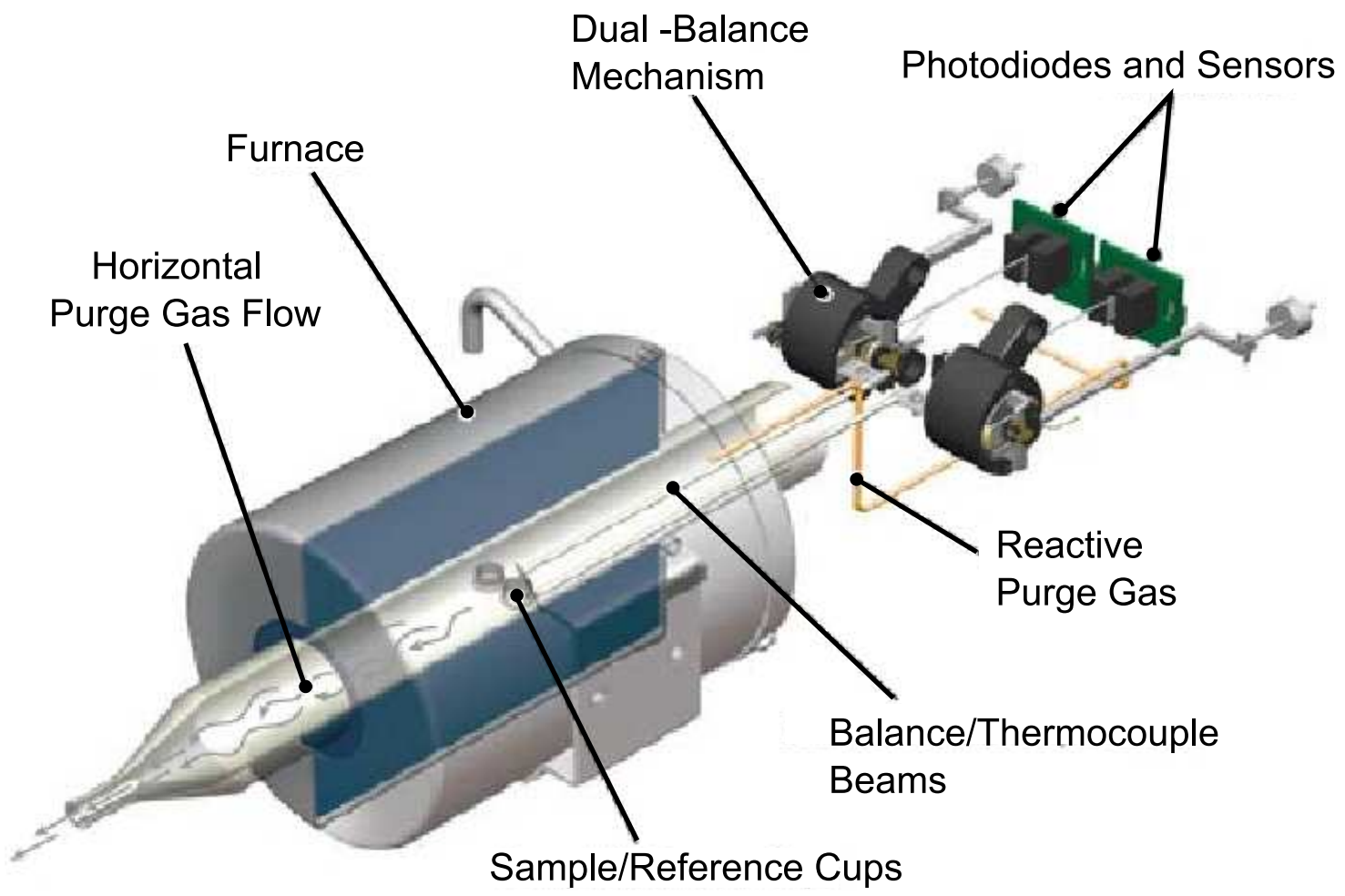

Figure 3.4: The SDTQ600 DSC/TGA instrument. Image taken from the specifications manual available on the TA Instruments website.

This instrument measures the differential weight between the sample pan and a control pan (see Figure 3.4). Hence, both the pans are carefully normalised to 
each other before the sample is placed into one. The advantage of this method is that very small weight changes up to $0.1 \mu \mathrm{g}$ can be resolved. Another useful advantage of the horizontal balance is that buoyancy effects - usually a problem with vertical balances - are eliminated. Temperature is measured with thermocouples attached to the bottom of the sample pans.

\section{Experimental details}

A/Prof Andy Edgar set up the instrument before each run including the calibration of the sample and reference pans. A concentrated pellet of nanotubes was collected in the sample pan from the centrifuged precipitate of a neat butylamine solution. The sample was allowed to dry in air for an hour to evaporate the liquid butylamine. Thermograms of the dried pellet were obtained under a horizontal nitrogen purge while the temperature was ramped from ambient to $500{ }^{\circ} \mathrm{C}$ at a rate of $10^{\circ} \mathrm{C} /$ minute.

\subsubsection{Annealing}

The SWNT films were annealed in a radiative oven in order to remove any traces of excess surfactant. The oven setup can be seen in Figure 3.5. The thin film was placed inside the glass tube and the tube was slid inside the furnace. The annealing temperature was set by pressing both the ' $\mathrm{T}$ ' button and one of the ' $\uparrow$ ' or ' $\downarrow$ ' buttons. The films were usually annealed to a set temperature of 320 ${ }^{\circ} \mathrm{C}$. The annealing temperatures were set $20-30{ }^{\circ} \mathrm{C}$ higher than required because the thermocouple feedback to the oven was separated from the sample space by the glass tube and indicated a lower temperature. Once the set temperature was attained, it was decreased manually to $20{ }^{\circ} \mathrm{C}$. The film removed from the oven when the temperature dropped below $200{ }^{\circ} \mathrm{C}$. A similar process was carried for the $500{ }^{\circ} \mathrm{C}$ and $100{ }^{\circ} \mathrm{C}$ anneals using set temperatures of $520{ }^{\circ} \mathrm{C}$ and $140{ }^{\circ} \mathrm{C}$ respectively. 


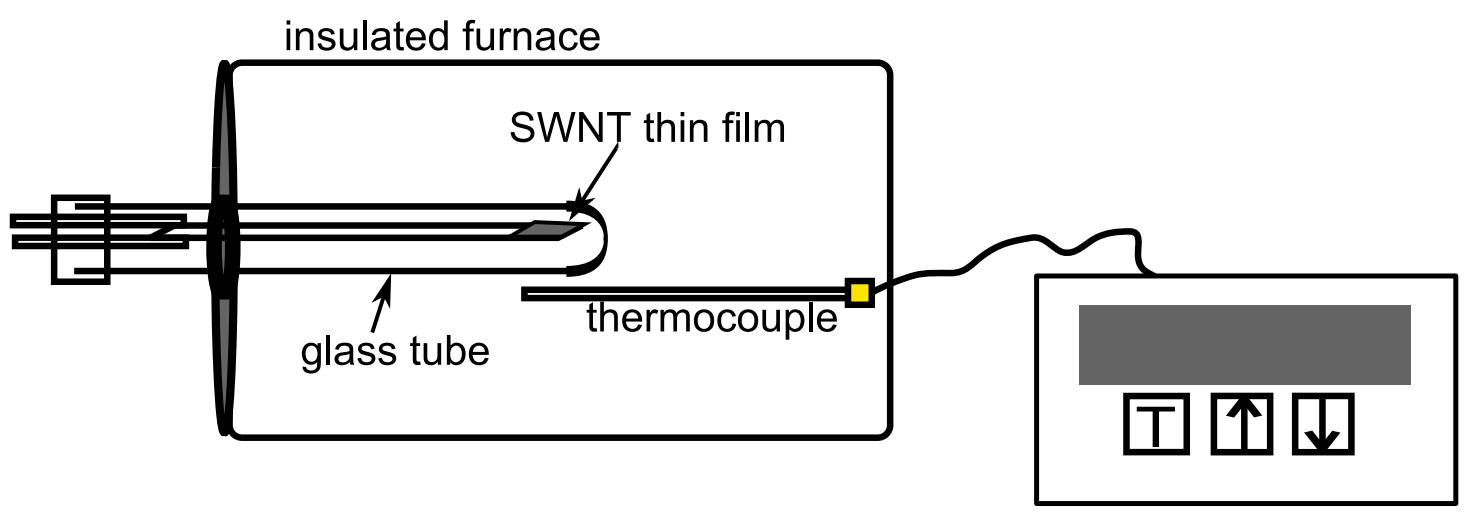

Temperature controller

Figure 3.5: Radiative annealing oven

\subsection{Raman spectroscopy}

In this study, a Jobin Yvon LabRam Raman spectrometer was used to obtain Raman spectra of SWNT thin films and solutions under the $632.8 \mathrm{~nm}$ (He-Ne laser) and $514.5 \mathrm{~nm}\left(\mathrm{Ar}^{+}\right.$laser $)$laser lines. An overview of the experimental apparatus and the conditions used to obtain spectra are outlined below.

\subsubsection{The Raman spectrometer}

Laser light from the sample is a combination of Rayleigh and Raman scattered light. A notch filter is used to reject the Rayleigh scattering. The holographic notch filter is a thin film of dichromated gelatin (DCG) that has a sinusoidal variation in refractive index with depth. The filter reflects the laser frequencies back through the objective while transmitting Raman-shifted light greater than 100 $\mathrm{cm}^{-1}$ from the laser line with high efficiency. Changing the angle of the incoming light incident on the notch between $0^{\circ}-10^{\circ}$ can transmit light from as low as 40 $\mathrm{cm}^{-1}$ from the laser line. This is done using spacers. For all the spectra from the $632.8 \mathrm{~nm}$ laser, a No. 7 spacer was used to extend signal collection to $100 \mathrm{~cm}^{-1}$.

The notch filter is used in single grating spectrometers for laser wavelengths 


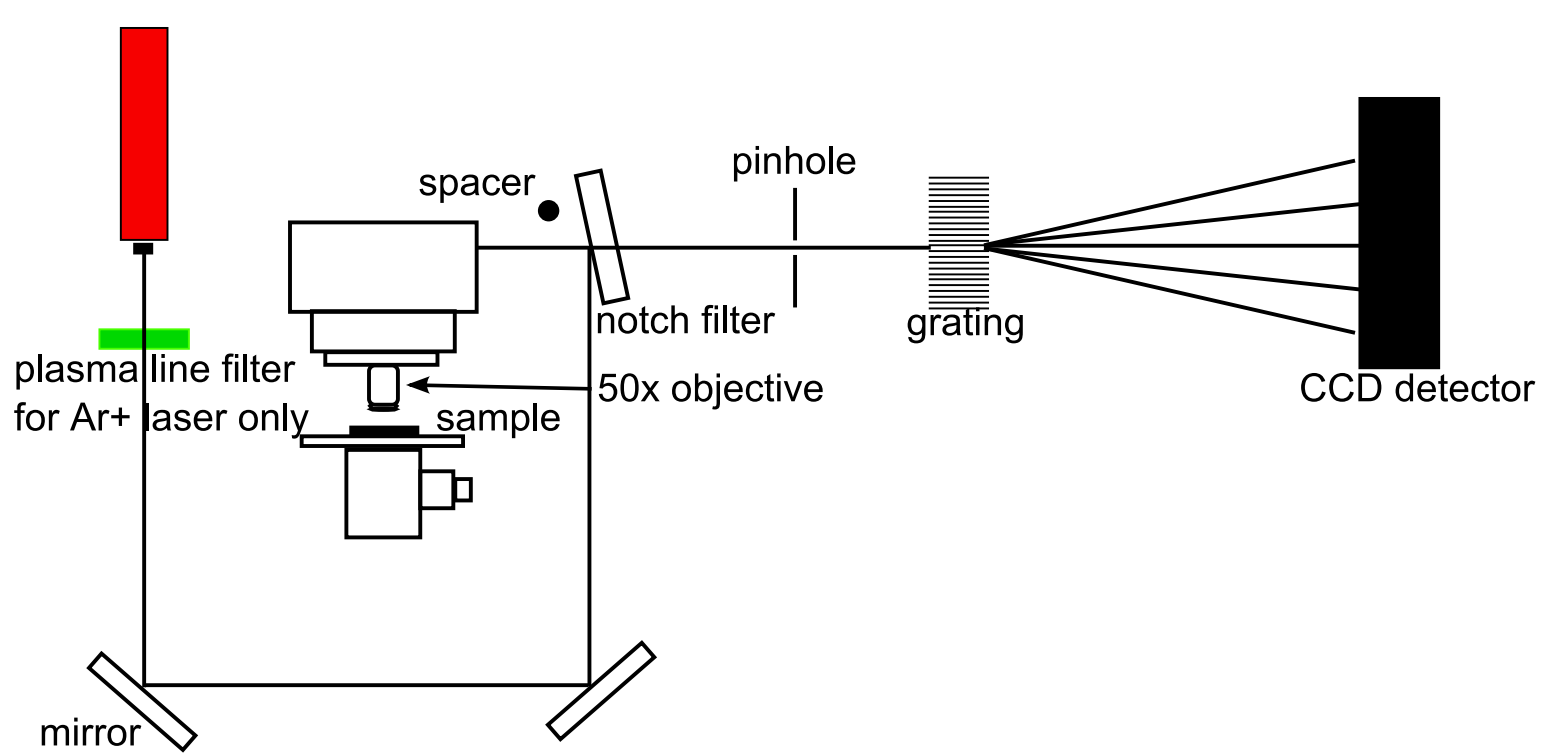

Figure 3.6: A schematic of the Jobin Yvon LabRam confocal Raman spectrometer used to obtain Raman spectra.

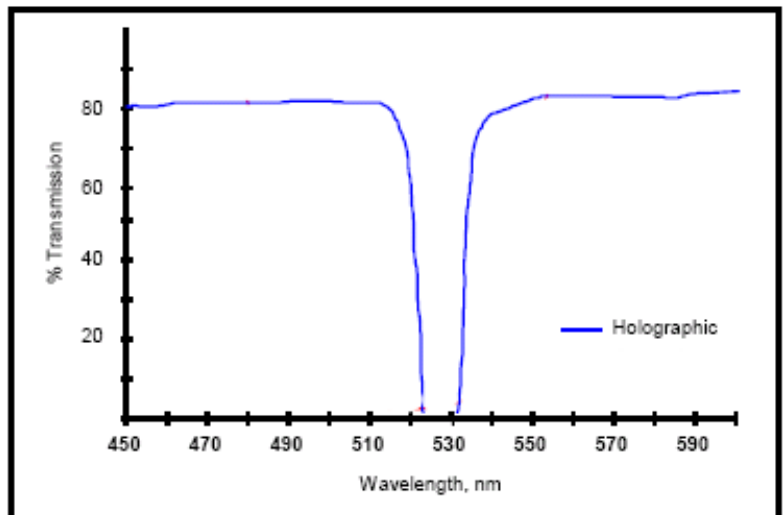

Figure 3.7: The holographic notch filter efficiently transmits all the Ramanscattered light while reflecting the Rayleigh-scattered light [83].

between 350 - $1350 \mathrm{~nm}$ to reflect only the laser line (Figure 3.7) and each laser line has a corresponding notch filter. Once isolated by the notch filter, the Raman scattered light is split into component wavenumbers by a diffraction grating. The gratings used with the LabRam range from 300 lines/mm to 2400 lines/mm. For the purposes of resolving the radial breathing modes within $0.3 \mathrm{~cm}^{-1}$ - features of the SWNT Raman spectrum between 150 and $400 \mathrm{~cm}^{-1}$ - the 1800 lines $/ \mathrm{mm}$ 
and 2400 lines/mm gratings were used for the $632.8 \mathrm{~nm}$ and $514.5 \mathrm{~nm}$ excitations respectively.

The light dispersed by the grating falls on the pixels of a charge-coupled device array (CCD). The incident photons generate electron-hole pairs in the silicon pixels of the CCD where the number of generated carriers are proportional to intensity. A computer software connected to the spectrometer builds a spectrum from the information. In order to reduce the dark current noise during long accumulation times - a feature of most silicon photodetectors - the CCD is cooled to cryogenic temperatures with liquid nitrogen. The spectrometer was calibrated against the $520 \mathrm{~cm}^{-1}$ Raman shift of crystalline silicon before every session.

\subsubsection{Confocal microscopy}

The confocal Raman microscope is a useful tool for spatial tuning of the collected signal. One of the main uses is to limit background fluorescence. To do this, light from the sample is passed through an adjustable pinhole aperture en route to the grating. The pinhole allows spatial control of signal collection. A small pinhole tightens the depth of signal collection closer to the focal plane (Figure 3.8).

The choice of microscope objective is very important for optimal signal collection. The numerical aperture of the objective determines the amount of light collected. This property is mainly related to the half-angle $(\mu)$ of the light collection cone. Objectives with wider light cones have larger numerical apertures and thus collect more light (see Figure 3.9). Another consequence of high numerical aperture is the increased resolution. Light from the sample can be viewed as an Airy disk (Figure 3.10) For larger numerical apertures, the collected light also contains many of the higher diffraction orders, thereby increasing the amount of sample information. Lower numerical apertures grab few higher orders leading to 


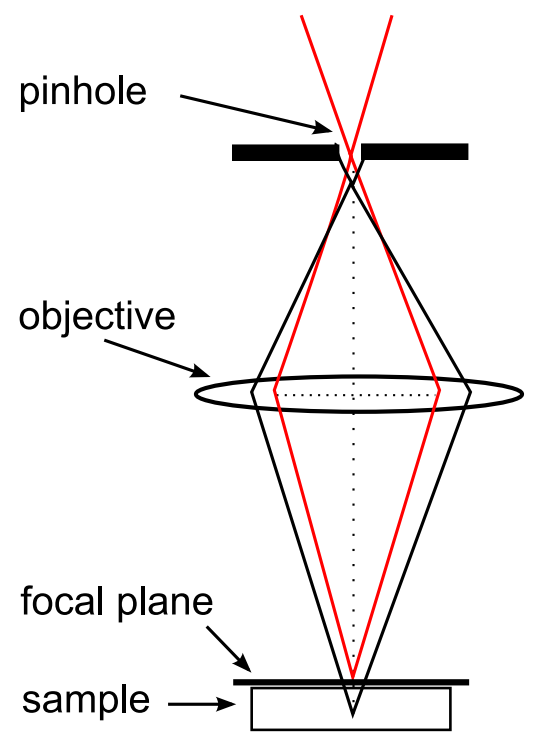

Figure 3.8: Spatial selection with confocal microscopy.
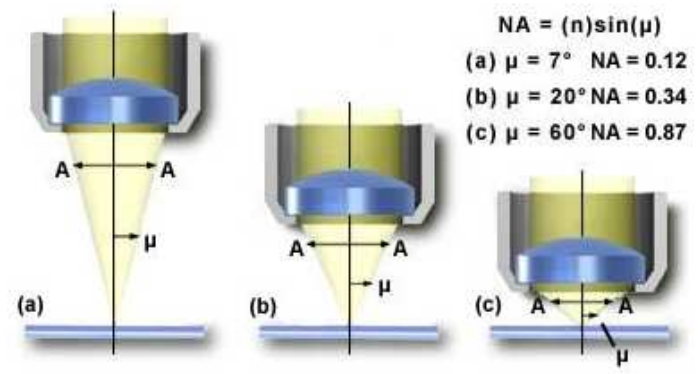

Figure 3.9: Variation in numerical aperture with half-angle, $\mu$. Image taken from the Nikon Microscopy U site [84].

poorer images. However in this study, the choice of objective was limited by the two different types of sample. While the $100 \times$ objective would have been ideal, the focal length was not compatible with the setup for the liquid samples. So, a $50 \times$ objective was chosen to obtain spectra from both solution and thin films. 


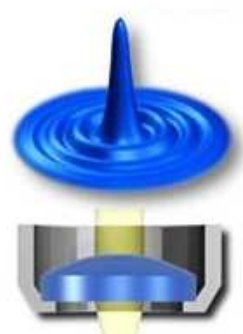

(a)

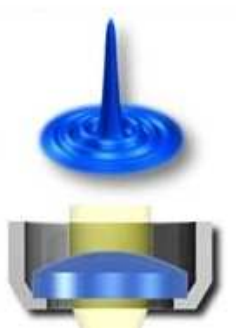

(b)

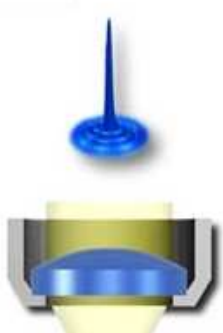

(c)

Figure 3.10: Airy disks for different numerical apertures. Image taken from the Nikon Microscopy U site [84].

\subsubsection{Solution samples}

The submersible lens was not used to obtain spectra from solution since it was likely that butylamine would dissolve the Teflon coating. Instead, $100 \mu \mathrm{L}$ of solution was pipetted into the cap of an epindorf and covered with a small glass coverslip to prevent evaporation. A schematic of this setup is shown in Figure 3.11. Solution spectra were obtained with a large pinhole of $400 \mu \mathrm{m}$ for sufficient spatial averaging. All spectra were accumulated over long integration times ( 5 - 7 minutes) for a high signal-to-noise ratio and averaged at least twice to minimise external artefacts like cosmic rays etc.

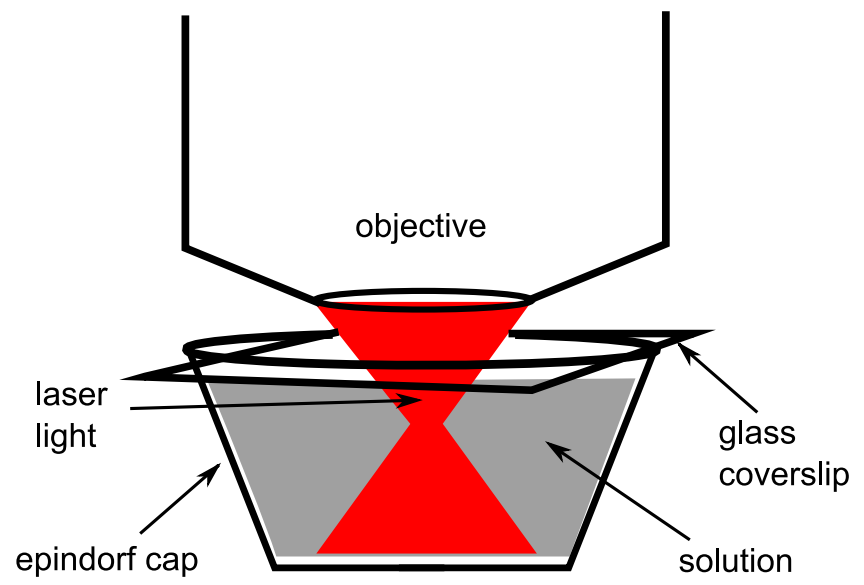

Figure 3.11: The experimental setup for collecting spectra from solution. 


\subsubsection{Solid samples}

The integration times for the solid samples were dependent on the density. For example, spectra from thick, SWNT powder were obtained in $30 \mathrm{~s}$ to one minute. On the other hand, spectra from thin networks were accumulated over several minutes (usually five). For the thin film samples, the pinhole was decreased to $100 \mu \mathrm{m}$ to tighten signal collection around the sample focal plane and cut down the fluorescence from the glass substrate.

\subsection{Low temperature conductivity}

Low temperature conductance measurements of SWNT thin films were obtained using a closed-cycle helium cryostat as well as a glass cryostat. The closed-cycle cryostat was used to obtain preliminary conduction data to $30 \mathrm{~K}$. The glass cryostat was used to obtain conductance data down to $1.3 \mathrm{~K}$.

\subsubsection{Closed-cycle cryostat}

The cold finger of the closed-cycle cryostat is connected to four pins which allowed 4-terminal conductivity measurements. In this study, only two pins were connected for 2-terminal measurements as shown in Figure 3.12. Two thermocouples: one sitting under the base of the sample mount and the other on the sample allowed for a qualitative monitoring of the thermal lag between the sample and the cold finger (Figure 3.12).

The sample was mounted on the cold finger using GE varnish and high vacuum cryogenic grease as shown in Figure 3.12. Wires from the contacts were soldered to the appropriate pins which lead to the Keithley 236 source measure unit (SMU) via guarded coaxial and triaxial cables. Once the sample was put in place, the sample chamber was isolated with a radiation shield and a second 


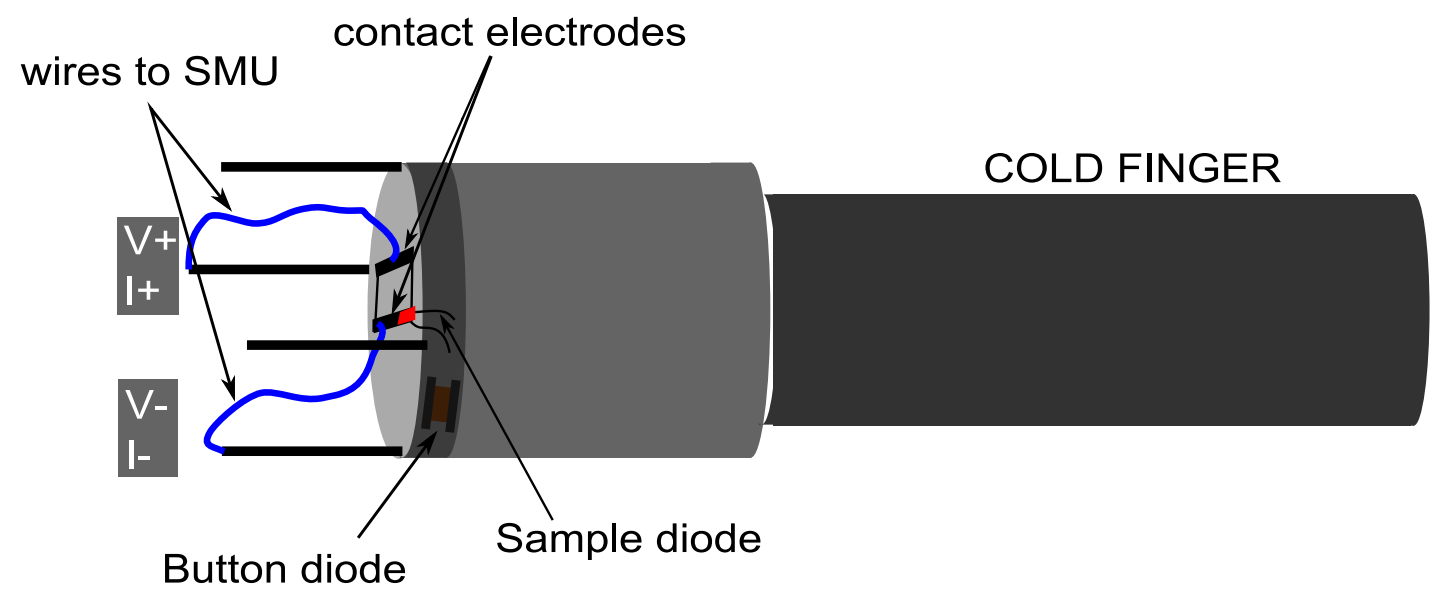

Figure 3.12: A schematic of the cold finger of the closed-cycle cryostat

cover. The chamber was then evacuated with a rotary pump and a diffusion pump.

The sample was cooled by cryogenic refrigeration utilising pressurised helium gas. Temperature control was achieved by modulating the cooling rate of the sample with a heater controlled by a Lakeshore 331 temperature controller. The temperature controller was in turn controlled by a LabView program (shown in Appendix A). The program allowed the user to set the various temperatures at which current-voltage (I-V) sweeps were taken by the SMU. For the convenience of the user, both sweep parameters and target temperatures could be set on the front panel of the program. The target temperatures were relayed to the temperature controller and the temperature ramping rates set automatically by the instrument. Proportional-integral-derivative (PID) values for the ramping rate were occasionally set on the temperature controller if the automatic setting was inadequate.

With this setup, I-V curves were reliably obtained from room temperature to $30 \mathrm{~K}$ and from $30 \mathrm{~K}$ back to room temperature. Plots of conductance as a function of temperature were generated from the the value at $10 \mathrm{~V}$. 


\subsubsection{Glass cryostat}

The glass cryostat is a completely different system to the closed-cycle cryostat. In this simple apparatus, the sample chamber sits in a liquid helium bath. The bath is thermally sealed from the atmosphere so the warm up is slow. A full run from $1.3 \mathrm{~K}$ to $300 \mathrm{~K}$ took around two days. The basic design is shown in Figure 3.13 .

The geometry of the sample mount allowed three samples to be measured per run (Figure 3.14). The samples were tacked on their mounts with GE varnish and electrical contacts to the sample were made with indium solder. Insulated constantan wires were used to connect the sample to the appropriate pins on the verro board. The wiring was set up according to the equivalent circuit shown

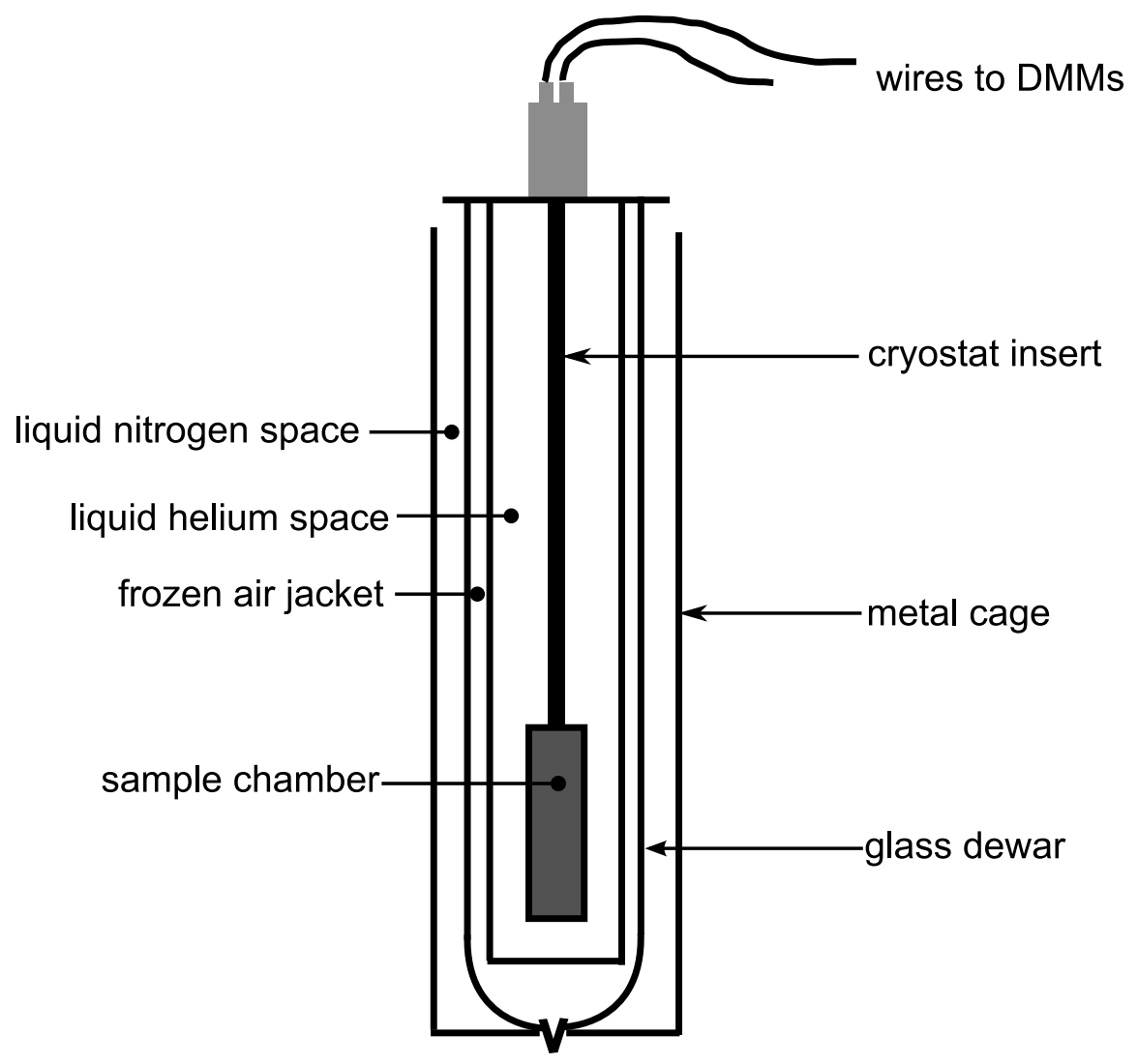

Figure 3.13: A schematic of the glass cryostat used for low temperature conductivity measurements. 


\section{Verro board}

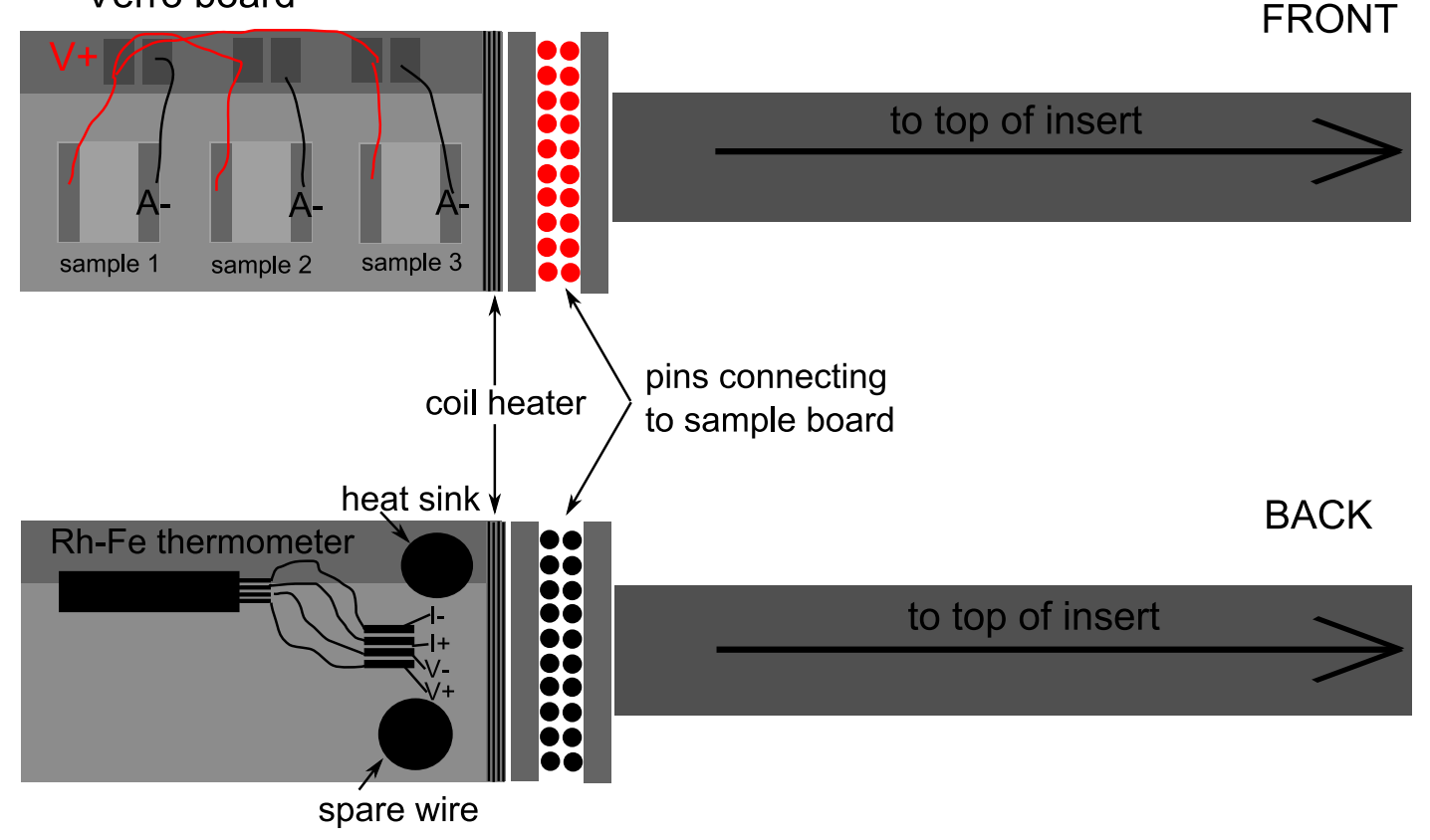

Figure 3.14: Diagram showing the front and back sides of the sample mount within the sealed chamber.

in Figure 3.15. This circuit was chosen so that the conductance data could be collected at a constant bias. A constant voltage bias was chosen over a constant current to avoid complications from voltage-dependent effects at low temperatures.

Wires from the sample were mechanically secured at various points with GE varnish. Once the samples were mounted, a radiation shield was screwed in place. A second cover which went over the shield was sealed with indium to isolate the sample chamber from the liquid helium bath and to prevent superfluid helium from entering the sample below $2.17 \mathrm{~K}$.

The insert (including the sample chamber) was placed into the glass dewar. The glass jacket between the liquid nitrogen space and helium space was then pumped out with a roughing pump and left with a 'finger-full' of air. This was done so that the air would freeze after the addition of liquid helium and become 


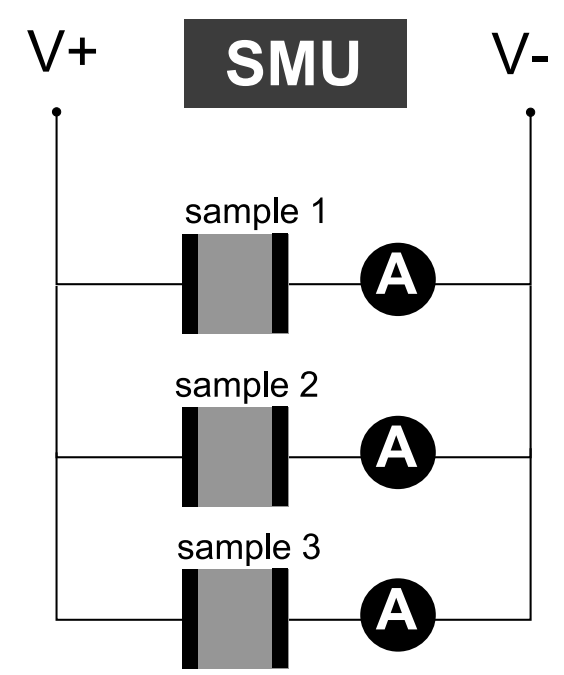

Figure 3.15: The equivalent circuit diagram for the three mounted samples.

an insulating jacket. The helium space was purged twice with helium gas to displace moisture. Data collection was started a few minutes before the liquid nitrogen was poured into the outer dewar. Cooling to $\sim 80 \mathrm{~K}$ took a few hours with sufficient levels of nitrogen.

The process of liquid helium transfer was begun once the rhodium-iron (RhFe) thermometer read below $90 \mathrm{~K}$. The specifics of this transfer are not detailed here. The transfer was carried out with the help of Dr. Chris Bumby and Dr. Ben Ruck. During the filling of the helium space, the temperature dropped rapidly to $4.2 \mathrm{~K}$ at which point the transfer was stopped. The helium space was then pumped on with a low throughput pump to reduce the temperature to $1.3 \mathrm{~K}$. To ensure that the sample temperature rose slowly, the sample chamber was evacuated with a rotary and a diffusion pump.

Conductance data was collected using the LabView program described in Appendix B. During runs with sufficient time and low resistance samples, the applied voltage was alternated in order between $50 \mathrm{~V}, 1 \mathrm{~V},-50 \mathrm{~V}$ and $-1 \mathrm{~V}$. 


\section{Chapter 4}

\section{Sample preparation}

\subsection{Dispersion}

SWNTs aggregate quickly due to strong van der Waals interactions $(\approx 500 \mathrm{eV}$ per $\mu \mathrm{m})$ between tubes [85]. Since dry flocculate cannot be manipulated, dispersions are essential for any studies or applications of SWNTs. However, researchers have struggled to create dispersions of SWNTs due to their unreactive sidewall: a consequence of low pyramidalisation angle $\left(\theta_{P}\right)$ [86]. The pyramidalisation angle is defined as the residual angle between the $\sigma$ and $\pi$ orbitals i.e. $\left(\theta_{\sigma \pi}-90^{\circ}\right)$. While $\theta_{P}$ is zero for $\mathrm{sp}^{2}$ carbon such as in graphene, curvature effects cause changes in the orbital hybridisation. For example, the spherical curvature of $\mathrm{C}_{60}$ results in a high $\theta_{P}$ of $11.6^{\circ}$. When compared to $\mathrm{sp}^{3}$ carbon with $\theta_{P}$ of $19.5^{\circ}$, we can see that $\mathrm{sp}^{3}$ hybridisation is energetically favoured. Hence, the $\mathrm{C}_{60}$ molecule is highly reactive. The $(5,5) \mathrm{SWNT}$ with a comparable radius to $\mathrm{C}_{60}$ has only half the angle of pyramidalisation and thus, a comparatively unreactive nature.

Initial preparations of SWNT dispersions could not overcome this inherent unreactive behaviour until surface functionalisation gained momentum as a method to boost reactivity. Functionalisation involves chemical modification of the side- 

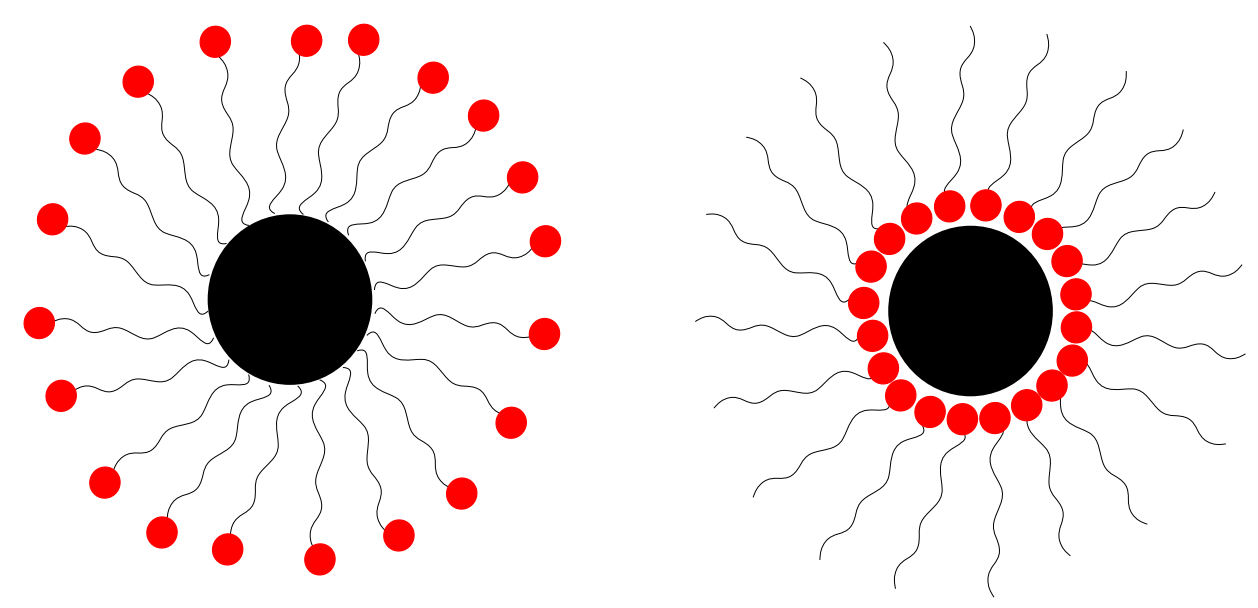

Figure 4.1: The micelle (left) and the reverse micelle (right) for ionic surfactants in water where the polar head is denoted by the red circle.

wall to create dangling moieties with high chemical reactivity (e.g. carboxyl groups). Such modified tubes are easily dispersed in solvents since the dangling bonds interact with the surrounding solvent to keep the nanotube floating in solution. Though this method is feasible, the harsh chemical treatment creates structurally defective tubes [68] while the covalent surface bonds destroy the unique electronic structure $[87,88]$.

An alternative method for preparing dispersions was borrowed from colloidal chemistry where surfactants kept the colloids afloat in solution. SWNTs encased within a protective layer of adsorbate disperse easily if the adsorbate interacts with both the tubes and the solvent.

Surfactants or surface active agents facilitate mixing between two otherwise immiscible fluids. Surfactant molecules are amphiphilic i.e. they have a hydrophobic (a hydrocarbon chain) and hydrophilic part (ionic or polar functionality). They form self-assembled structures in solution above a critical concentration since interaction of the part of the molecule with the opposite polarity to the solvent is thermodynamically disfavoured. A two dimensional cross-section of the common spherical micelle is shown in Figure 4.1. Surfactant interactions with 
the solvent and the SWNTs do not occur immediately but need to be encouraged using physical agitation like sonication.

\subsubsection{The dispersion process}

SWNT aggregates can be separated by shear mixing or sonication. The unbundling of nanotubes can be visualised as an 'unzipping' mechanism [89] where the bundles are physically unzipped from the ends during exfoliation (Figure 4.2 (a)). When the ends are frayed, there is sufficient room for the surfactant to squeeze in between and adsorb on the nanotube surface (Figure 4.2 (b)). The surfactant penetrates further down the tube with prolonged sonication (Figure 4.2 (c)) until the two parts separate completely (Figure $4.2(\mathrm{~d})$ ). The tubes are kept isolated by the surfactant which coats the sidewalls and prevents re-aggregation.

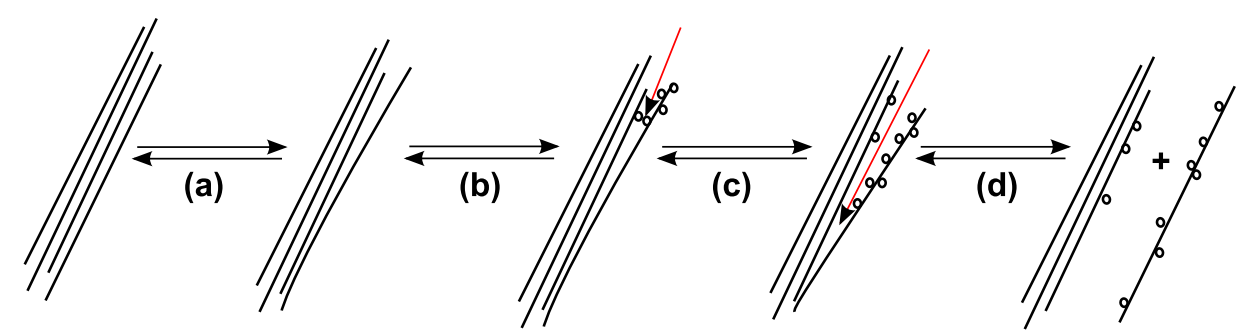

Figure 4.2: (a) The start of the unzipping process. (b) Introduction of surfactant into the gap created by sonication. (c) Continued penetration of the surfactant into the increasing gap. (d) Unzipping complete. Adapted from [89].

Undispersed material such as aggregates, carbonaceous impurities and remnant catalyst can be pelleted out by centrifugation leaving only the dispersed tubes in solution.

The unbundling process (Figure 4.2) has been monitored by both fluorescence [90] and Raman ([91, 51] spectroscopy. A marked increase in the signal from individual SWNTs and a sharpening of spectral features confirmed that the Van Hove transitions of individual SWNTs become clearer with increasing sonication time. 
That is, the unbundling of aggregates improves with longer sonication. However, prolonged sonication also causes mechanical deformation such as buckling and reduced lengths [86].

The efficacy of the dispersion process - proportional to the fraction of individual SWNTs in solution - is dependant on the surfactant as well as the duration and power of sonication and centrifugation. Dispersion quality is often limited by instrumental capabilities and repeated sonication and centrifugation are necessary to increase the fraction of individual nanotubes. A short sonication time in a powerful sonicator and long centrifugation times at high speed were shown to significantly decrease the number of bundles within the dispersion [60]. However, the parameters for sonication and centrifugation differ for each system since the dynamics of nanotubes in dispersions is particularly dependent on the chemistry between the SWNTs and the surfactant as well as the solvent-surfactant interaction.

\subsubsection{Surfactants in SWNT dispersions}

\section{Aqueous dispersions}

Ionic surfactants are effective in dispersing nanotubes in an aqueous environment. The most prevalent in nanotube dispersions are sodium dodecyl sulphate (SDS) [90] followed by similar detergents like sodium dodecylbenzene sulphonate (NaDDBS) [92] and, various bile salts such as sodium cholate (SC), sodium deoxycholate (SDOC) and sodium taurodeoxycholate (STDOC) [93, 94]. Though all these surfactants are ampiphilic, their interactions with SWNTs are not identical.

Detergent molecules have a generic structure: a long hydrocarbon chain (either an alkyl group such as SDS or more complex carbon rings) capped with an 
ionic functionality. SWNT-SDS dispersions prepared with a surfactant concentration above the critical micellar concentration were assumed to form micelles [90] or hemimicelles [92] around the nanotube. However, these possibilities have been refuted by results from small angle neutron scattering [95] that point to random orientation of surfactant molecules on the nanotube surface.

Physical adsorbates do not perturb the nanotube electronic structure. For example, SDS is a benign adsorbate that interacts weakly without altering any of the intrinsic properties of SWNTs. However, adsorption strengths vary depending on the relative chemistry of the adsorbant and adsorbate. In the case of NaDDBS, the hydrophobic part includes a benzene ring that initiates $\pi$-stacking interactions with the delocalised $\pi$ electrons of the SWNT [92].

\section{Organic dispersions}

The low volatility of aqueous dispersions is a distinct disadvantage for fabricating thin films. Organic solvents with high volatility and reactivity can also be engineered for chiral or electronic type selectivity and volatile dispersions. The literature reviewed in the following section is relevant to the butylamine/THF system used in this study.

Early studies utilised polar, organic solvents such as dichlorobenzene (DCB), dichloroethane (DCE) [96], dimethyl formamide (DMF) [15] and n-methyl pyrrolidone (NMP) [97] as the sole component in dispersions. Among these, solvents with a high electron donation ability such as DMF and NMP, showed superior dispersions. DMF was shown to disperse both carboxyl-functionalised and purified SWNTs. While these solutions tended to be short-lived, the shelf life of these poor dispersions were found to improve drastically upon the addition of aliphatic amines [15]. 
Alkylamines of varied chain lengths show similar amphiphilic characteristics to ionic surfactants. Mimicking the two-component formula of aqueous dispersions i.e. a surfactant and solvent; organic analogues with a polar head group can effectively disperse both functionalised and untreated SWNTs. Initially, the high dispersion efficiency of this formula was believed to be due to strong Coulombic interactions between the amino group and the carboxyl moieties that lead to the formation of zwitterionic dangling bonds [98, 15]. However, this model fails to describe the mode of dispersion for non-functionalised nanotubes in alkylamine and THF as shown by Maeda et al. [99, 17, 18, 19].

The physisorption of amines on the SWNT sidewall accounts for the dispersion of both untreated and treated nanotubes. The room temperature adsorption of amines indicates the absence of an activation barrier for adsorption [100]. If present, such a barrier would support chemisorption. Theoretical calculations place the adsorption energy of methylamine around $0.1 \mathrm{eV}$ [18].

The properties of amine-assisted dispersions are ideal for film fabrication. As discussed earlier, film fabrication is encumbered by the low volatility of solvents and extensive post-deposition treatment to remove residual surfactant or surface functionalities. Since thin films are the main focus of this study, ease of fabrication is a desirable quality.

\subsubsection{The dispersion procedure}

The generic dispersion procedure involved sonicating $\sim 15 \times 10^{-5}$ g of raw HiPCO SWNTs (purchased from Carbon Nanotechnologies) in a 7:1 ratio of tetrahydrofuran (THF) and distilled n-butylamine. The total amount of solvent was kept 
constant at $12 \mathrm{~mL}$ for experimenting with different solvent ratios. The mixture was sonicated in a $15 \mathrm{~mL}$ glass bottle with a solvent-proof bottle cap and an external covering of Parafilm. Typically, sonication time lasted between 3 - 6 hours in a gentle bath sonicator. The duration and power of sonication was kept low since long nanotubes were required for an entangled network. Also, gentle sonication prevented the disintegration of nanotubes into amorphous carbon.

Following sonication, the solution was centrifuged at 14,000 r.p.m. until the undispersed material precipitated in a compact pellet at the bottom of the epindorf. This process usually took between 30 - 60 minutes. The resultant supernatant was used for fabricating SWNT films.

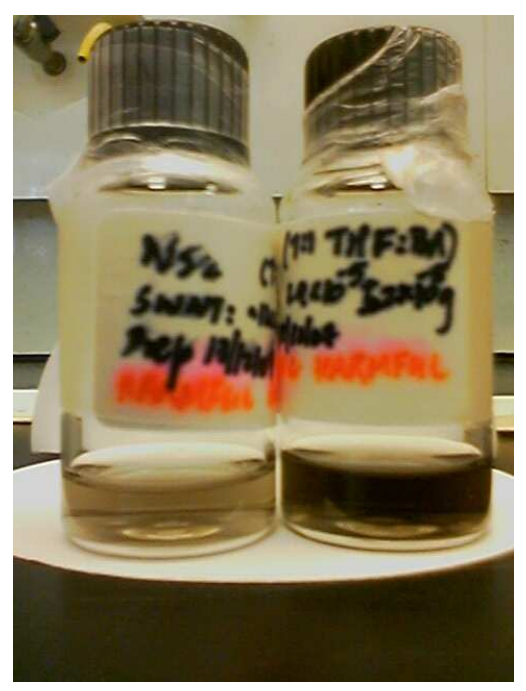

Figure 4.3: Two dispersions of different SWNT concentrations prepared with a $7: 1$ solvent to surfactant ratio. The solution on the left was prepared according the amounts described in Section 4.1.3 while the one on the left was made with twice the initial amount of SWNTs.

SWNT dispersions using the above process were stable for months. We speculate that this stability is due to the compatibility of butylamine with both the SWNTs and the THF. Butylamine and THF are highly miscible solvents since their polarity, density and CED are all of similar magnitude (see Table 4.1). The 


\begin{tabular}{|l|l|l|}
\hline Parameter & Butylamine & THF \\
\hline \hline Density $\left(\mathrm{g} / \mathrm{cm}^{3}\right)$ & $0.74[101]$ & $0.889[102]$ \\
\hline Dipole moment $(\mathrm{D})$ & $1.4[103]$ & $1.63[104]$ \\
\hline Cohesive energy density $(\mathrm{MPa})[105]$ & 338.56 & 345.96 \\
\hline \hline
\end{tabular}

Table 4.1: Values of the physical parameters that determine miscibility for butylamine and THF.

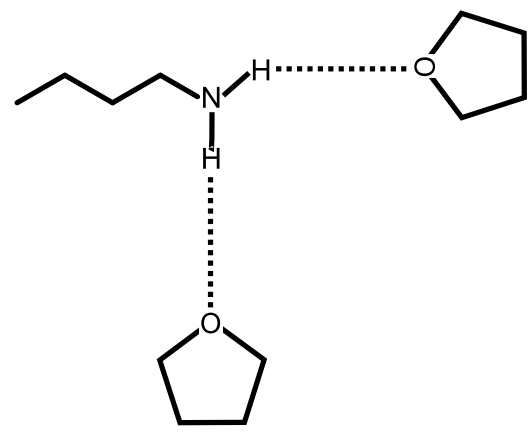

Figure 4.4: Schematic of the hydrogen bonding between butylamine and THF.

solvents interact via hydrogen bonding. The hydrogens on the amine functionality form weak covalent bonds with the oxygen on the THF as shown in Figure 4.4.

\subsection{Thin film fabrication}

\subsubsection{Film deposition}

Thin films of SWNTs were fabricated using a drop-drying method. The standard procedure involved drying a $100 \mu \mathrm{L}$ droplet on a clean $12 \mathrm{~mm}$ x $12 \mathrm{~mm}$ glass coverslip or quartz substrate. The films were dried in a variety of environments including the open air, purged fumehood and under a beaker. The majority of the films studied in this project were dried on a cork bung covered with a clean beaker.

Drop-cast films fabricated in the manner still contained varying amounts of residual surfactant and solvent. Films that appeared clean to the naked eye had $\sim 65 \%$ transparency while films with semi-opaque coating had a transparency of $\sim 30 \%$. The detrimental effects of remnant surfactant on the optoelectronic 


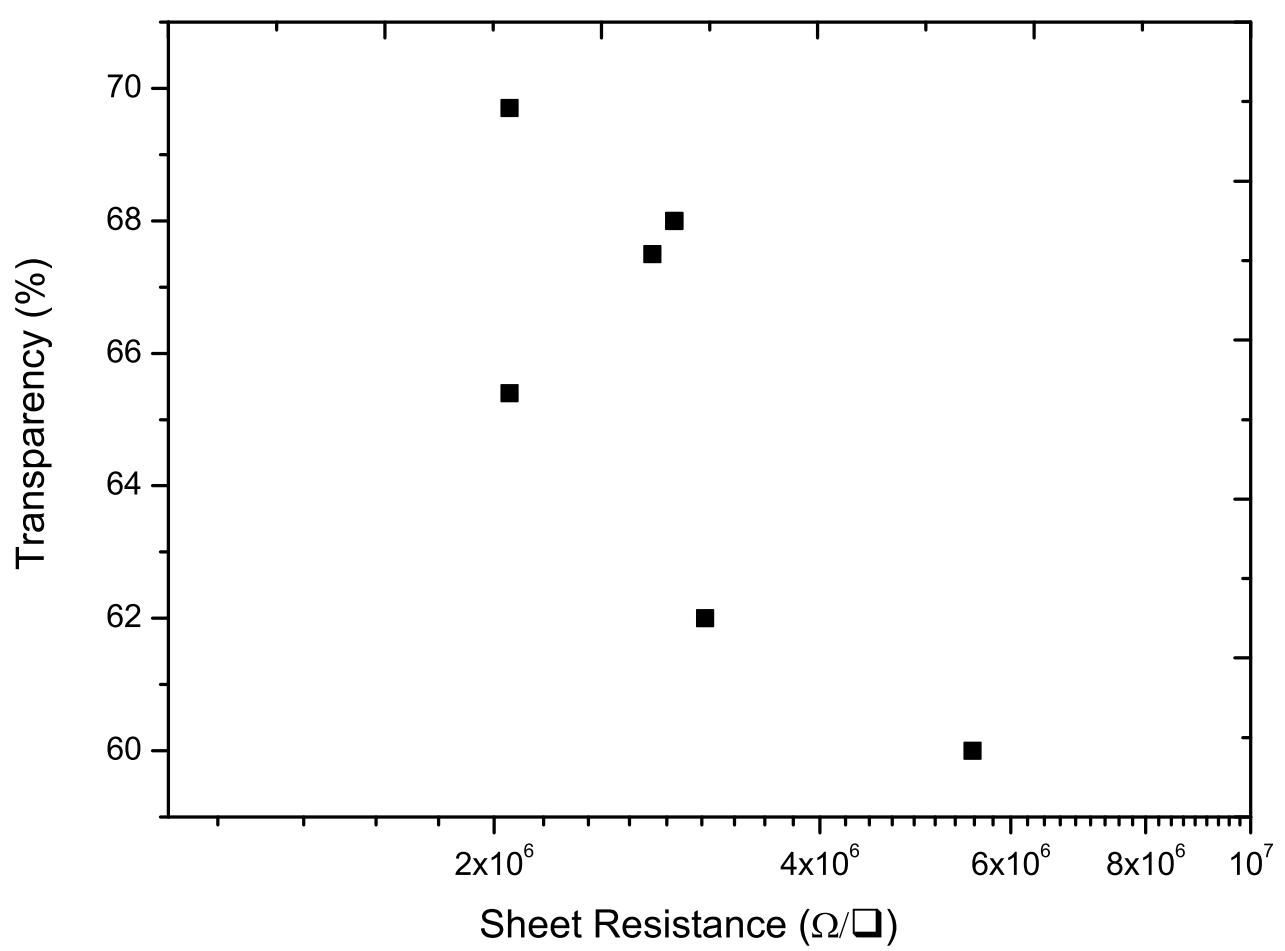

Figure 4.5: Optoelectronic properties of unannealed SWNT networks prepared from the same batch of solution.

properties of SWNT films have been reported previously as the cause of reduced transparency and conductivity [106].

Inconsistent sheet resistance for films fabricated from the same batch of solution are shown in Figure 4.5. The pattern in the figure shows a decreasing trend in the optoelectronic properties. Figure 4.6 shows the SEM image of an unannealed film with a network that resembles an entangled mesh. The dark patch is solvent residue pyrolysed by the electron beam. Hence, our results confirm the degrading effect of surfactant and solvent residue on optoelectronic properties.

The deposition patterns were found to be strongly dependent on the state of the SWNT dispersion. Older solutions produced films of inferior quality. One disadvantage of volatile solvents is that slow evaporation due to long term usage significantly alters the ratio of solution to SWNTs. The quality of the disper- 


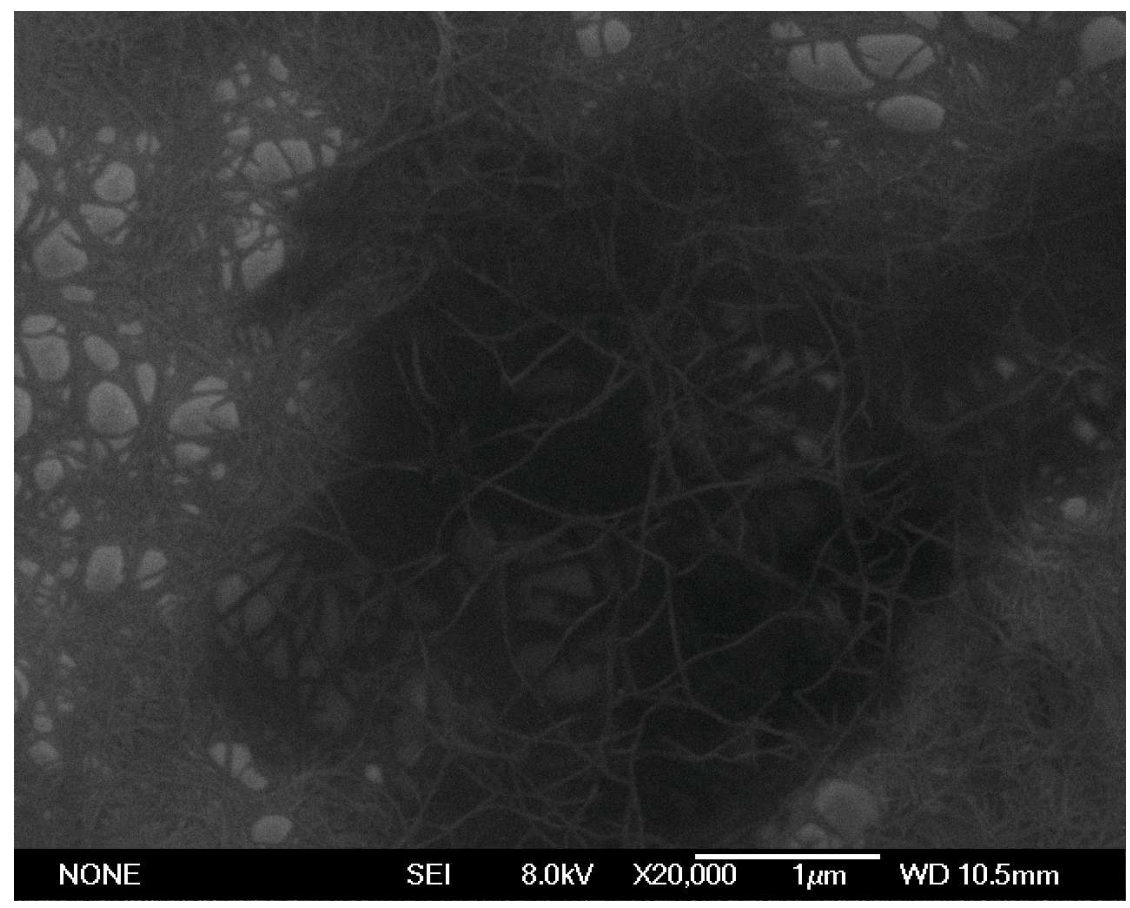

Figure 4.6: SEM image of an unannealed film. The dark patch separating the SWNT layers is pyrolysed solvent.

sion is compromised and the resulting films form cracks and fat ropes due to sensitivity of the drop-drying method. During the drying process, the SWNTs in solution assemble at the air-droplet interface. Since older dispersions contain larger bundles, the customary drying process is disrupted by the formation of even larger aggregates as the solvent evaporates. Instead of settling into a neat, woven morphology; the reduced flexibility and mobility of the clumps cause rips in the film or thick rope-like structures. However, despite the problems associated with the drop-drying method, thin films of SWNTs could be produced with little effort within 30 minutes - 1 hour.

\subsubsection{Improvements to optoelectronic properties}

In the earlier section, some of the shortcomings of as-prepared films were discussed. We now address improvements to optoelectronic properties by postdeposition treatment. 


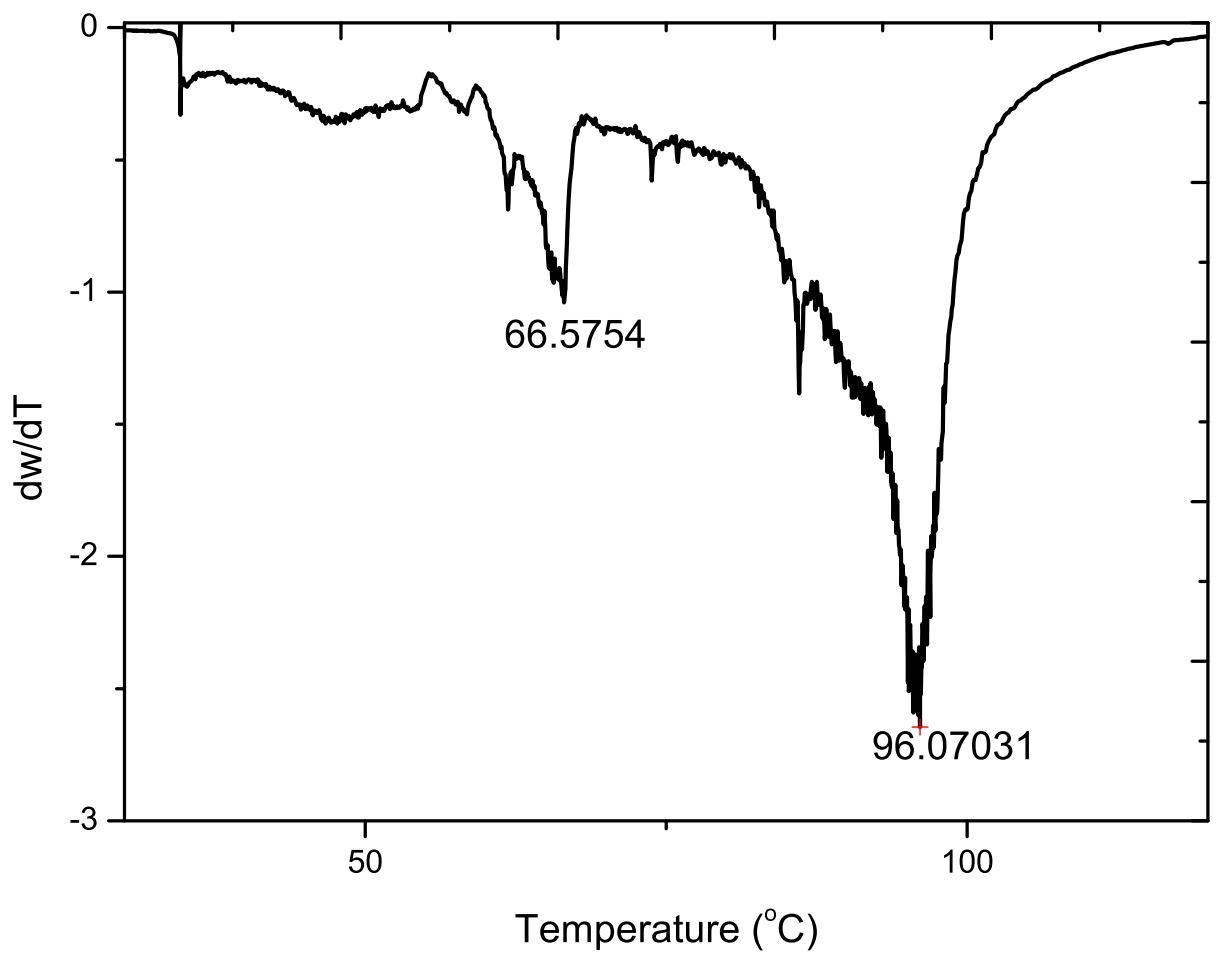

Figure 4.7: Differential thermogram showing the peak associated with evaporation of water. The two smaller peaks at $66^{\circ} \mathrm{C}$ and $80{ }^{\circ} \mathrm{C}$ are due to evaporation of THF and butylamine.

Films deposited from aqueous dispersions using detergent as surfactants (e.g. SDS or SC) need to undergo several cycles of meticulous washing to remove residual surfactant. The advantage of small, organic molecules is that they can be efficiently removed by annealing leading to vast improvements in optoelectronic properties.

Since solvent and surfactant residue was the cause of poor optoelectronic properties, a TGA study of butylamine-coated SWNTs was performed to determine the temperature of desorption. The derivative of the thermogram (Figures 4.7 and 4.8) shows three main peaks at $96{ }^{\circ} \mathrm{C}, 267^{\circ} \mathrm{C}$ and $439{ }^{\circ} \mathrm{C}$. The small peaks at $66{ }^{\circ} \mathrm{C}$ and $\sim 80{ }^{\circ} \mathrm{C}$ are due to the evaporation of THF and butylamine respectively. The first major peak at $96{ }^{\circ} \mathrm{C}$ is attributed to water. 


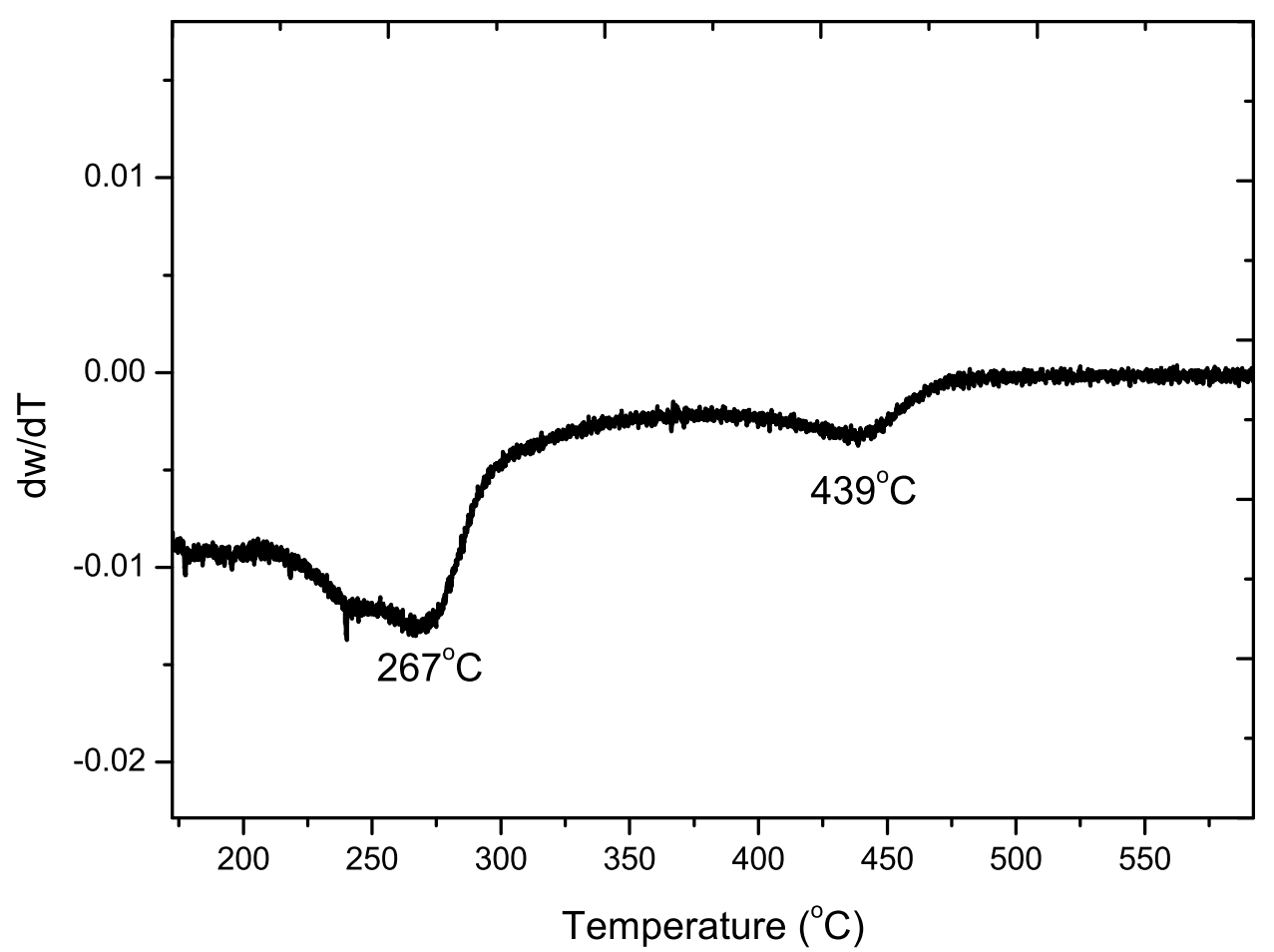

Figure 4.8: Differential thermogram showing the two smaller peaks at $267^{\circ} \mathrm{C}$ and $439{ }^{\circ} \mathrm{C}$

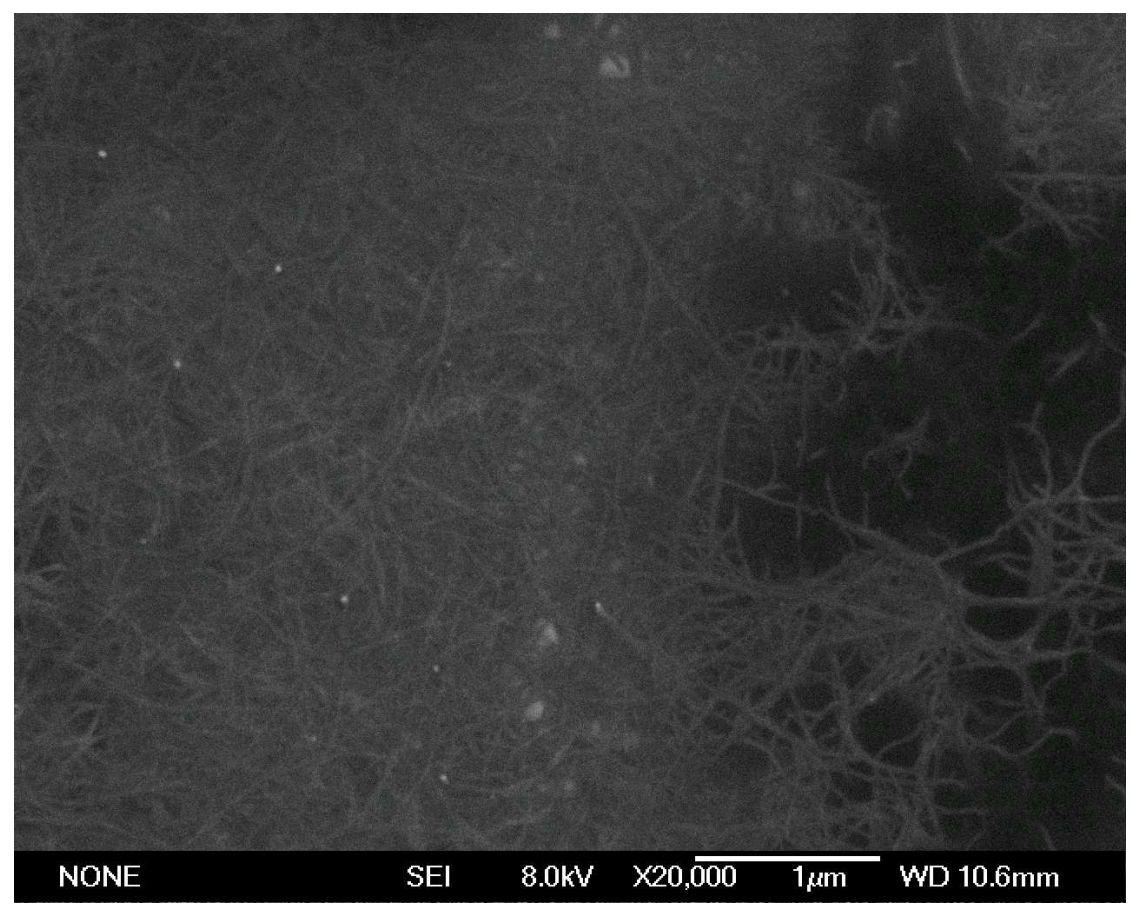

Figure 4.9: SEM image of a film annealed at $140{ }^{\circ} \mathrm{C}$. The dark patch separating the SWNT layers is pyrolysed solvent. 


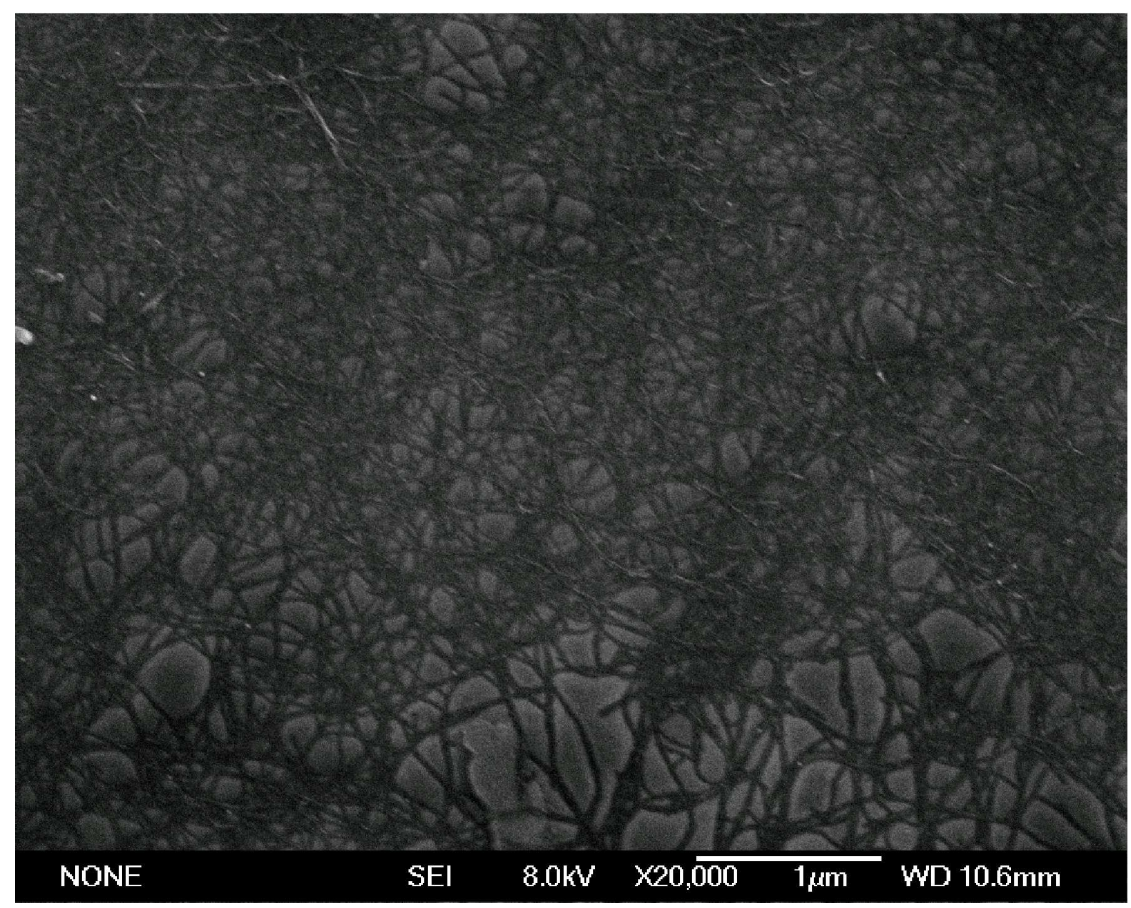

Figure 4.10: SEM image of a film annealed at $300{ }^{\circ} \mathrm{C}$.

From the low temperature solvent loss, it seems that the solvent residues can be removed by annealing to above $100{ }^{\circ} \mathrm{C}$. Figure 4.9 shows a SWNT network annealed at $140{ }^{\circ} \mathrm{C}$ at a magnification of $20,000 \times$. The image is very similar to the unannealed network shown in Figure 4.6. If the residues were removed during the anneal, the dark patches would have disappeared. The existence of these patches indicates the butylamine is bound in some way to the nanotubes.

The second peak is almost three times the boiling point of butylamine $\left(78^{\circ} \mathrm{C}\right)$. Figure 4.10 shows the SEM image of a SWNT network annealed at $300{ }^{\circ} \mathrm{C}$. There are no visible markers of solvent residue indicating that they have been removed by the annealing, leaving behind a network of pristine SWNTs. The removal of surfactant also dramatically improves transparency from $60 \%$ to over $80 \%$ and causes a reduction in the sheet resistance by at least two orders of magnitude.

Further proof that it is the surfactant butylamine that remains bound to the 


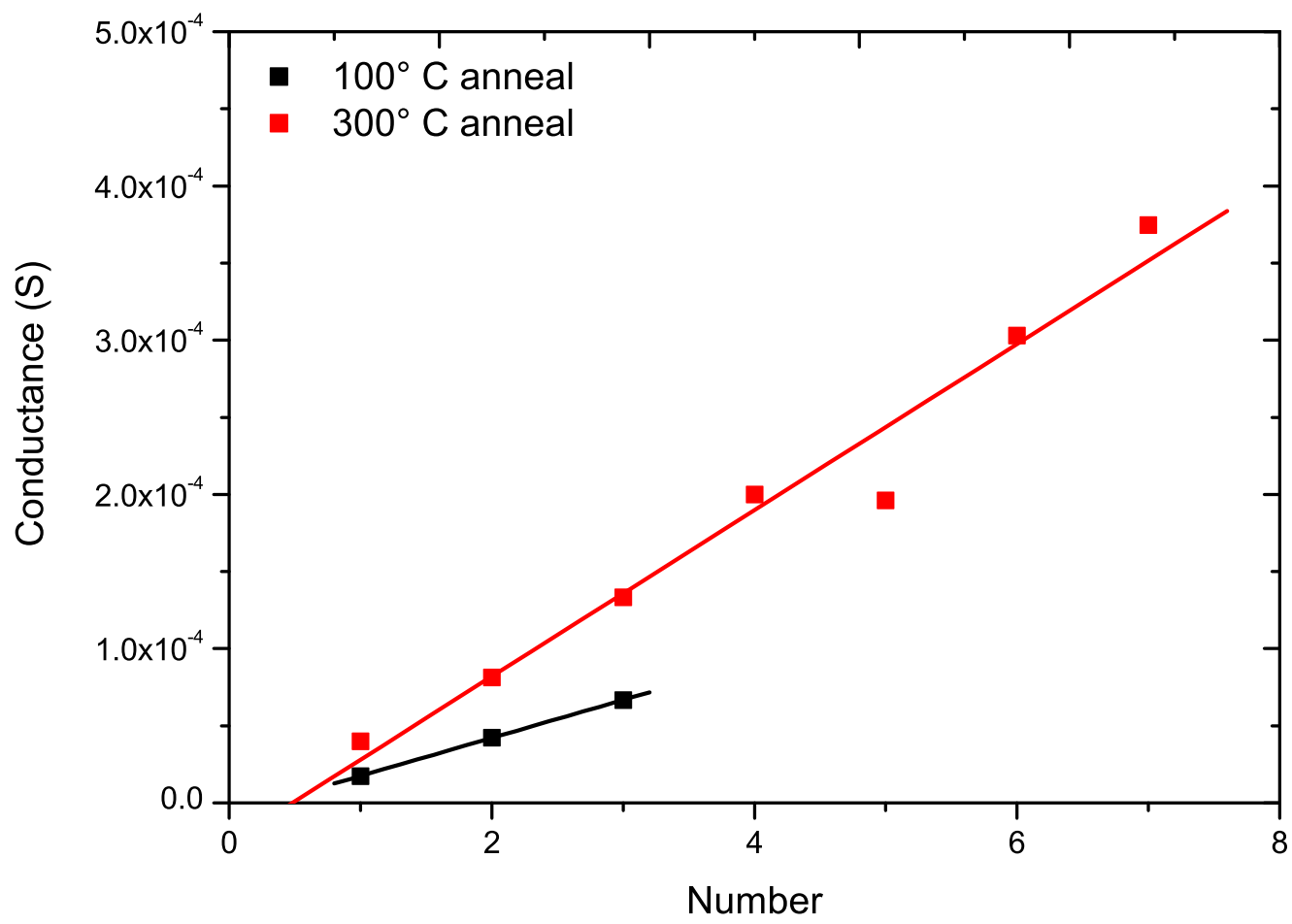

Figure 4.11: Improvement in the conductance after successive drop-casting and annealing.

nanotube sidewall can be seen in Figure 4.11. Figure 4.11 shows the changes in the conductance after successive drop-casting and annealing though only the conductance after annealing is plotted. The improvement in conductance after annealing at $300{ }^{\circ} \mathrm{C}$ is greater than that after the $100{ }^{\circ} \mathrm{C}$. Hence, the peak at $267{ }^{\circ} \mathrm{C}$ can be associated with the non-zero adsorption energy of the amine. The temperature equates to an adsorption energy of $45 \mathrm{meV}$. This value qualitatively matches the value of $\sim 0.1 \mathrm{eV}$ for methylamine adsorption on SWNTs [18].

Though optoelectronic properties are improved by annealing, the inconsistency continues even in annealed films. Figure 4.12 shows a plot of optical transparency against sheet resistance for annealed networks. Without the large intertube barriers due to residual surfactant, bigger variations in conductivity occur in annealed networks. Parameters like film thickness, fraction of metallic nanotubes, 


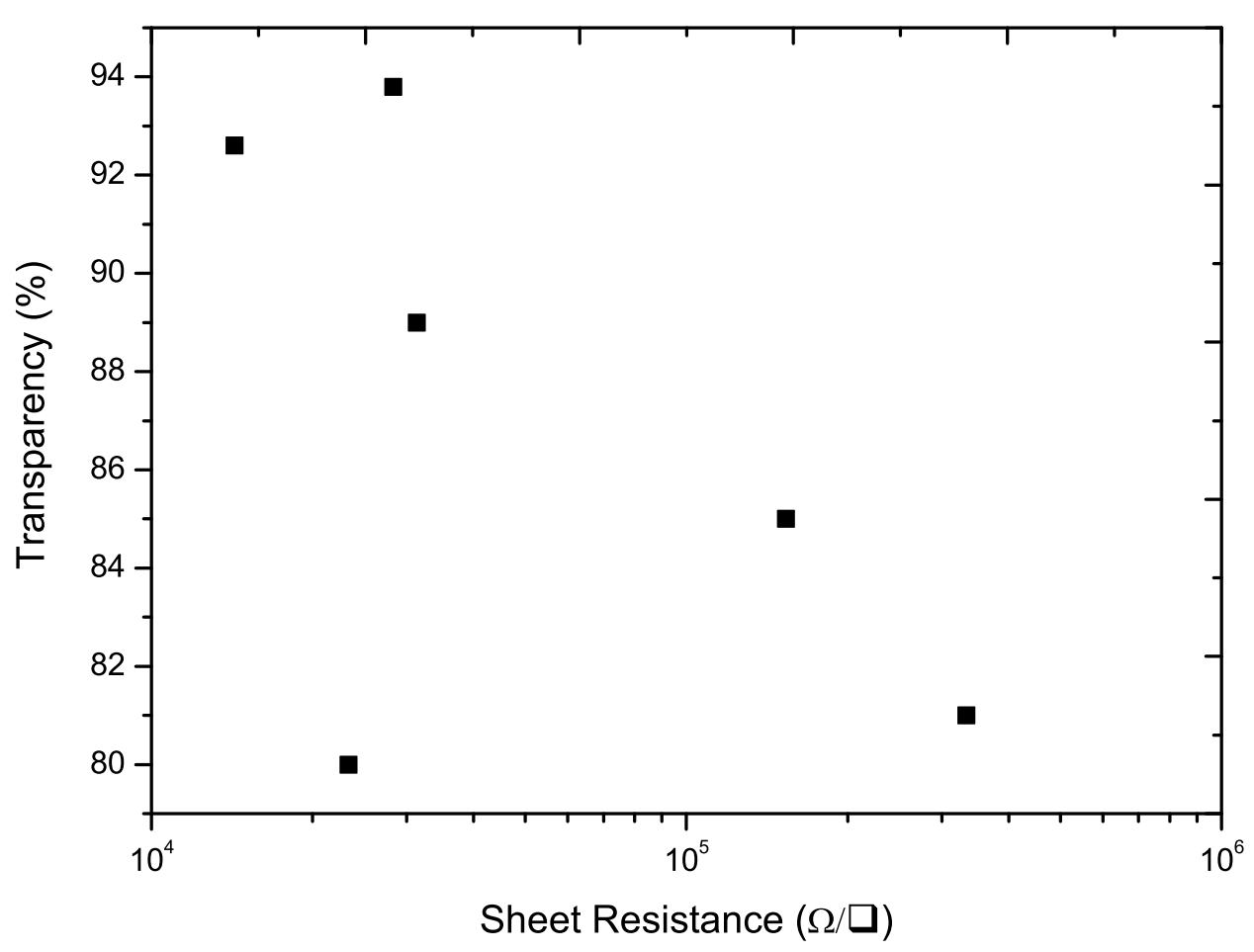

Figure 4.12: Optoelectronic properties of annealed SWNT networks.

variable morphology and oxygen doping [107, 108] all influence conductivity in these networks. However, these factors are difficult to control leading to a large variability in the optoelectronic properties.

Figure 4.13 shows that the sheet conductance can be improved by repetitive drop-casting and annealing. However, this improvement is at the cost of film transparency. Conductance increases due to the larger number of percolation networks in the successively thicker films. By the same regard, transparency is reduced after each round due to increasing film thickness.

The peak at $439{ }^{\circ} \mathrm{C}$ corresponds to combustion of SWNTs. The TGA study performed by Li et al. showed a similar peak at $\sim 420{ }^{\circ} \mathrm{C}$ which they attributed to nanotube combustion [109]. The removal of these tubes breaks the interconnectivity of the network as seen in Figure 4.14 and the network is essentially 


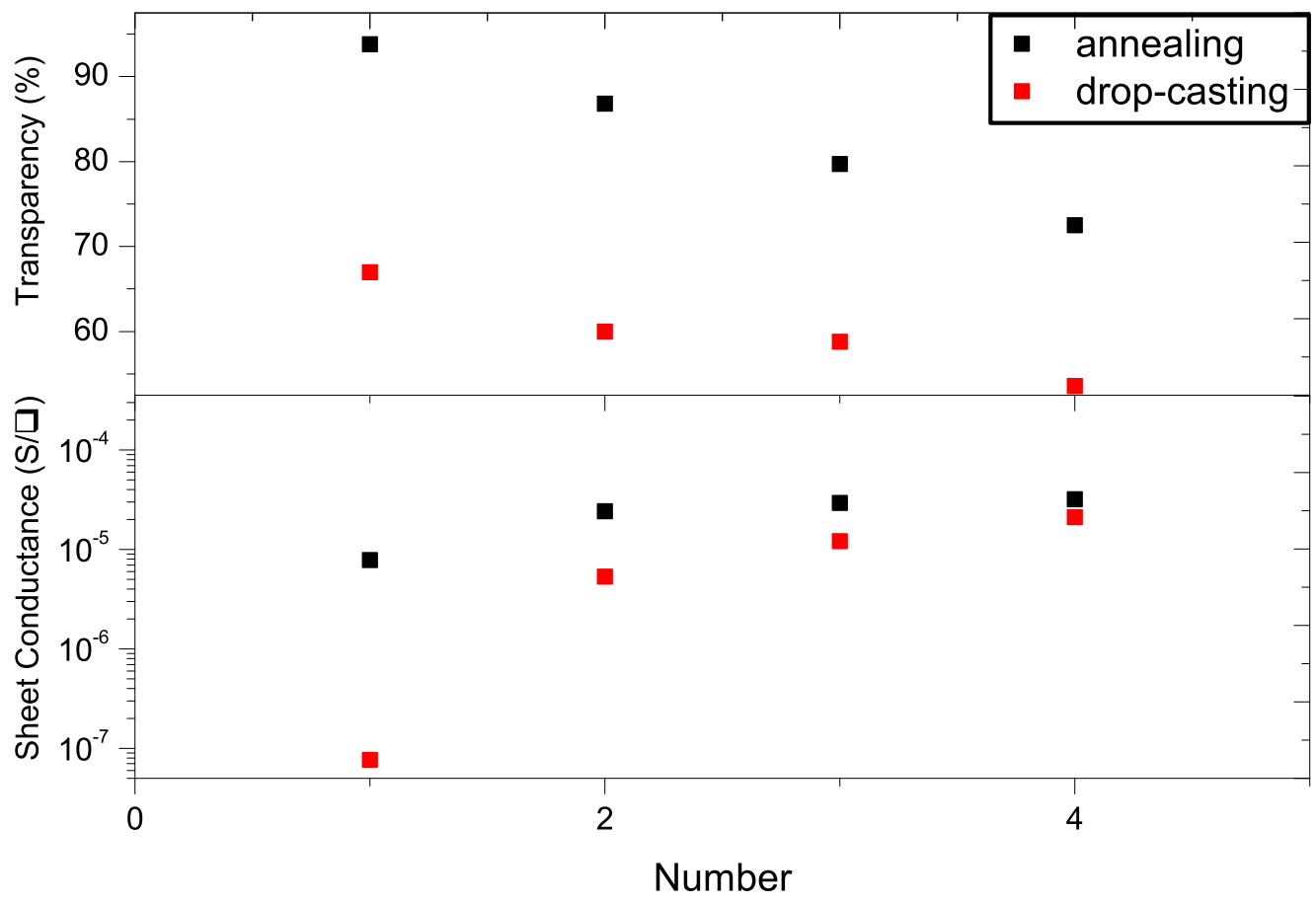

Figure 4.13: Optoelectronic properties after successive drop-casting and annealing.

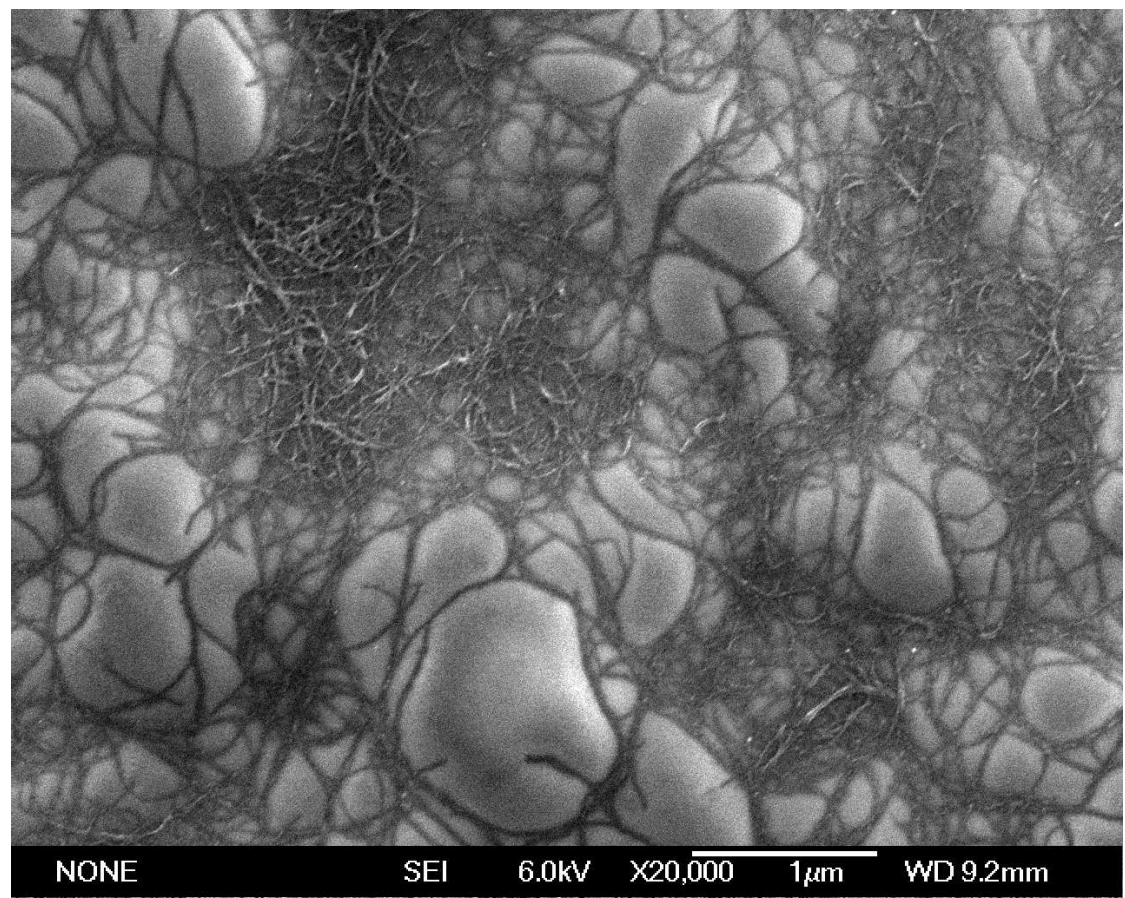

Figure 4.14: SEM image of a thin film annealed at $500{ }^{\circ} \mathrm{C}$. 
non-conducting. The raised bumps seen in the SEM image is the rough surface of the glass substrate.

\subsection{Conclusion}

The optoelectronic properties of unannealed thin films depend greatly on the amount of residual solvent and surfactant. Hence, there is great variability in the optical transparency and sheet conductance. Annealing SWNT films to 300 ${ }^{\circ} \mathrm{C}$ removes all solvent residue but oxygen is thought to dope the network. The variability in the level of doping, morphology and fraction of metallic tubes all contribute to the inconsistency in the optoelectronic properties of annealed films. 


\section{Chapter 5}

\section{Raman characterisation}

The transformation of nanotubes from raw powder to transparent thin films was presented in Chapter 4. The consequences of the physical treatments were only characterised using macroscopic properties such as the network morphology, sheet conductivity and transparency. But, even small changes in macroscopic features reflect more significant changes at a microscopic level. Raman spectroscopy probes nanotube systems in the micron dimensions since the laser spot can be focused to $\sim 1 \mu \mathrm{m}^{2}$. The four main spectral features of the nanotube Raman spectrum can be used to infer changes in specific physical properties.

The region below $350 \mathrm{~cm}^{-1}$ of the SWNT Raman spectrum contains the radial breathing modes or RBMs. As described in Section 2.2.3, each individual RBM peak has a one-to-one relationship with an individual nanotube. The diameter, chiral angle, and chiral index can all be assigned from the peak frequency using experimental Kataura plots. Significant changes in the physical properties of the nanotube sample are reflected in the observed RBMs as well as the intensity and frequency of each peak. Hence, physical and chemical treatments which alter the electronic properties of nanotubes or the distribution of nanotubes in the sample can be tracked by monitoring the RBMs. In the following sections, we monitor the 
changes in the RBMs after every step of the fabrication process. The results are presented in the order of thin film preparation outlined in Sections 4.1.3 and 4.2.2 i.e. commencing with the effects of dispersion and ending with the consequences of the annealing treatment.

\subsection{SWNT dispersions}

As per the dispersion procedure outlined in Section 4.1.3, raw HiPCO powder is sonicated with a 7:1 mixture of butylamine and THF. The large aggregates in the powder are exfoliated into smaller bundles by a combination of mechanical separation induced by sonication and the capping layer formed by the butylamine. Any remaining large bundles in the sonicated solution are removed by centrifugation. In the final supernatant, the nanotubes exist in a different environment and aggregation state from the raw powder. To investigate these changes, RBM spectra of raw HiPCO powder and a centrifuged dispersion were obtained using the method described in Section 3.3.

Figures 5.1 and 5.2 show the RBM spectra of raw powder and a centrifuged butylamine/THF dispersion taken with the $514.5 \mathrm{~nm}$ and $632.8 \mathrm{~nm}$ lasers respectively. Within each figure, the solution and powder spectra not only exhibit differences in intensity but also in the observed RBMs (highlighted with a red circle). These changes could reflect preferential adsorption of the surfactant on specific nanotubes. The other possibility is that a different subset of nanotubes are bought into resonance due to changes in bundle size between the powder and solution. We examine both these possibilities in detail in the following sections. 


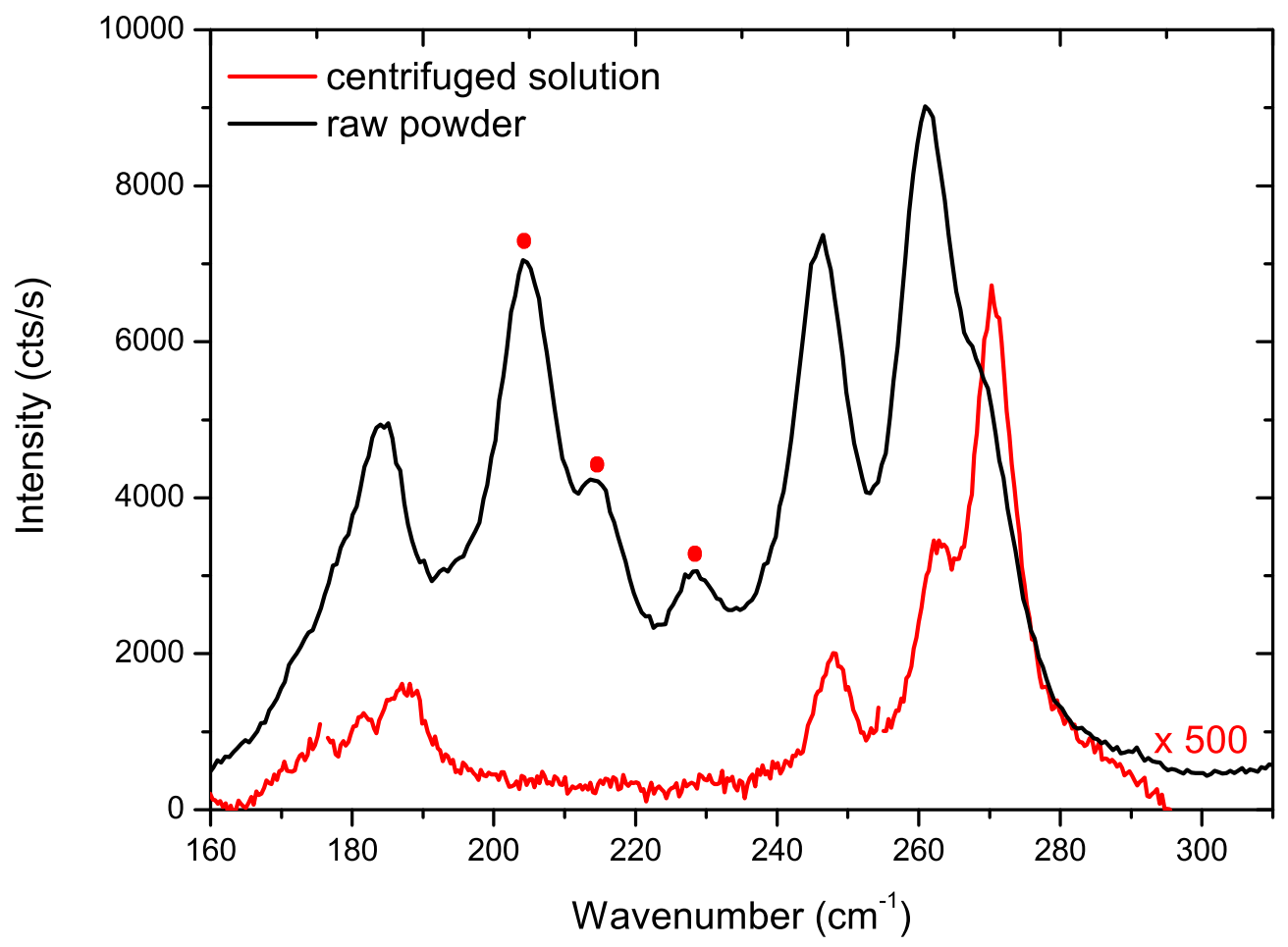

Figure 5.1: Spectra of raw HiPCO powder and a SWNT dispersion prepared with a 7:1 ratio of THF:butylamine taken under the $514.5 \mathrm{~nm}$ laser. The peaks marked with a red circle are only observed in the powder.

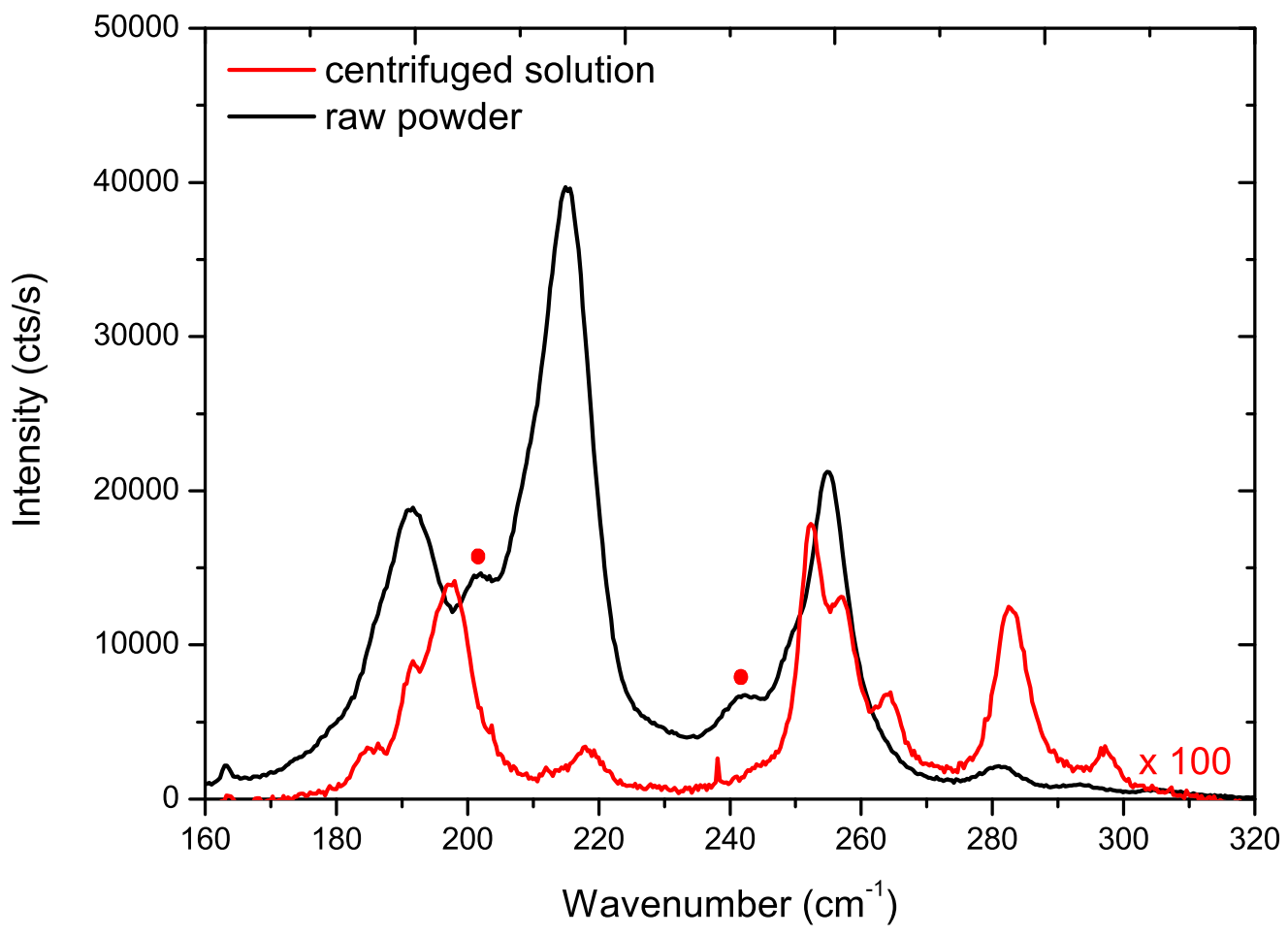

Figure 5.2: Spectra of raw HiPCO powder and a SWNT dispersion prepared with a 7:1 ratio of THF:butylamine taken under the $632.8 \mathrm{~nm}$ laser. The peaks marked with a red circle are only observed in the powder. 


\subsubsection{Surfactant selectivity}

Surfactant molecules act as a spacer preventing the nanotubes from agglomerating in solution. As discussed in Section 4.1.1, the surfactant-nanotube interaction is crucial for creating a stable dispersion. Much of the early work in this field assumed a constant interaction energy between the two interacting species. Recently, several studies found that the interaction actually varied depending on the electronic nature of the nanotube. Studies of this nature made a high impact (and still do) since they presented an achievable solution for separating nanotubes. Most synthetic routes for producing SWNTs cannot be tuned for electronic type. Hence, post-synthesis sorting is the only viable route for catering to the growing number of SWNT applications requiring specifically metallic or semiconducting characteristics.

The nature of the nanotube-surfactant interaction can be used to predict the preference of a given surfactant. For example, surfactants with aromatic groups (like naphthalene) that interact via $\pi$ stacking preferentially adsorb on metallic tubes [110]. Similarly, diazonium reagents covalently bond with metallic nanotubes since they can only form the intermediate complex with tubes that have a non-zero DOS at the Fermi level [88]. On the other hand, both metallic [18, 19] and semiconducting [98] selectivity for amines were reported in the literature. This seeming inconsistency arises from changes in the nanotube-amine interaction as a function of electronic doping of the semiconducting nanotubes.

Alkylamines act as charge transfer agents: donating electrons via the lone pair on the nitrogen atom. Though they are less powerful than strong electron donors like potassium which can n-dope already p-doped tubes [111], studies of aminated semiconducting tubes have shown an almost complete repression of hole conduction [112]. Charge transfer to the electron-deficient (p-doped) nanotubes 
led to weakened conductivity which was found to be reversible when the amines were desorbed [112].

For a while, electron transfer to doped nanotubes was thought to be the root mechanism behind amine selectivity. Chattopadhyay et al. reported selective adsorption by a long chain primary amine (octadecylamine) for carboxyfunctionalised semiconducting tubes prepared by the HiPCO method [98]. Semiconducting tubes are p-doped during the functionalisation process. Raman studies performed by Samsonidze et al. confirmed this selectivity as well as a previously unobserved preference for smaller diameter semiconducting SWNTs [16]. Kim et al. conjectured a theoretical model based on the relative redox reactivity of p-doped and metallic SWNTs to explain the electronic selectivity [113]. Their mechanism for selective solubilisation of semiconducting tubes was proposed on the basis that electron deficient tubes are reduced (i.e. they gain electrons) by the adsorbed amine. Since metallic tubes show more resistance to reduction, they are subsequently precipitated out.

The theory of Kim et al. became redundant when the empirical findings it was based on were contradicted by reports of consistent metallic selectivity for as-prepared SWNTs [18]. In view of this new result, a revised theoretical model was put forth to explain the change in amine selectivity. The interaction difference (ID) model [18] explains the conflicting results as being due to changes in the amine-SWNT interaction energy with the level of p-doping. The model predicts metallic selectivity for undoped SWNTs and a shift in this selectivity towards semiconducting tubes above some critical value of p-doping.

The ID theory is not based on quantifiable parameters and it only offers a possible explanation for the observed selectivity. So, in order to address the issue 


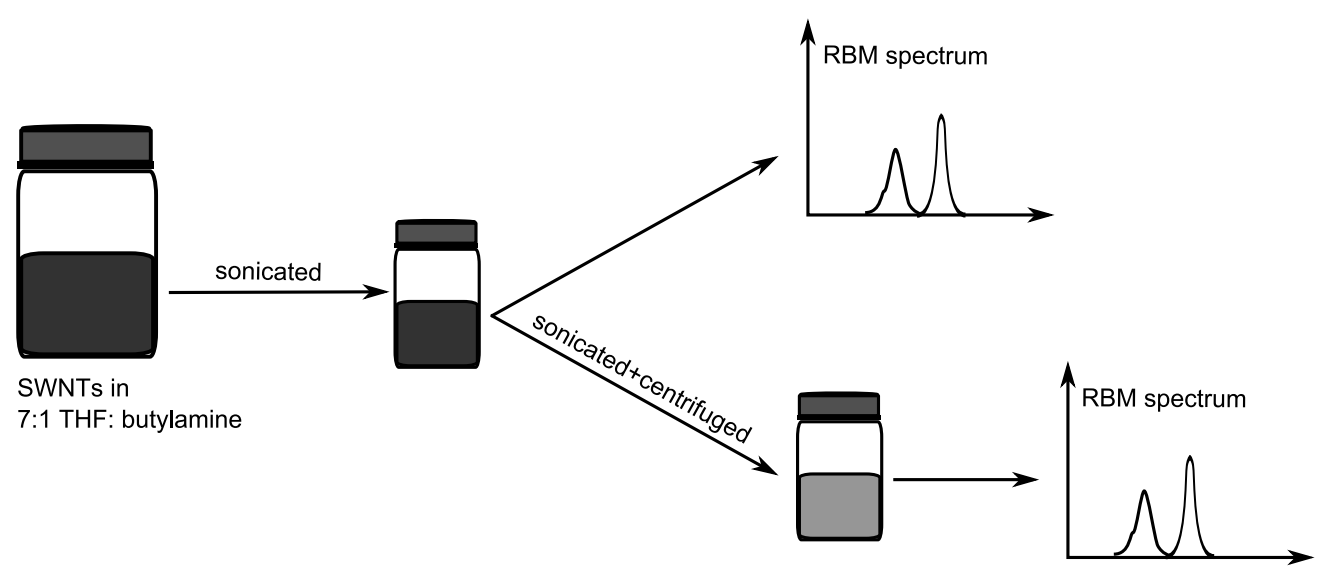

Figure 5.3: The process for studying selectivity in SWNT-butylamine dispersions. The sonicated solution is separated into two fractions: one fraction is centrifuged and the other remains uncentrifuged. RBM spectra are obtained from both these solutions.

of selective amine adsorption in our system, we conducted a brief study using Raman spectroscopy. Two batches of sonicated 7:1 butylamine:THF solutions were compared with the resultant supernatant after centrifugation (process is depicted in Figure 5.3). This comparison is based on the rationale that the sonicated solution - though well dispersed - still contains the same tubes as the raw sample. However, in the centrifuged solution only the SWNTs selected by the amine would remain.

Figures 5.4 and 5.5 depict RBM spectra from two batches of sonicated and centrifuged dispersions collected under the $632.8 \mathrm{~nm}$ and $514.5 \mathrm{~nm}$ lasers. The RBM intensities are normalised to the $282 \mathrm{~cm}^{-1}$ peak and $270 \mathrm{~cm}^{-1}$ for the 632.8 $\mathrm{nm}$ and $514.5 \mathrm{~nm}$ laser lines respectively. Peak intensities and positions are consistent between both solution types. The jagged edges are the only observed discrepancy in the solution spectra. These arise due to poor signal-to-noise ratio stemming from a relatively weak SWNT signal that cannot overcome excitations from the large volume of solvent. The spectra taken with the $514.5 \mathrm{~nm}$ laser show additional, broad peaks at $280 \mathrm{~cm}^{-1}$ and $220 \mathrm{~cm}^{-1}$ from butylamine. The higher energy $514.5 \mathrm{~nm}$ laser induces more extraneous excitations compared to the lower 


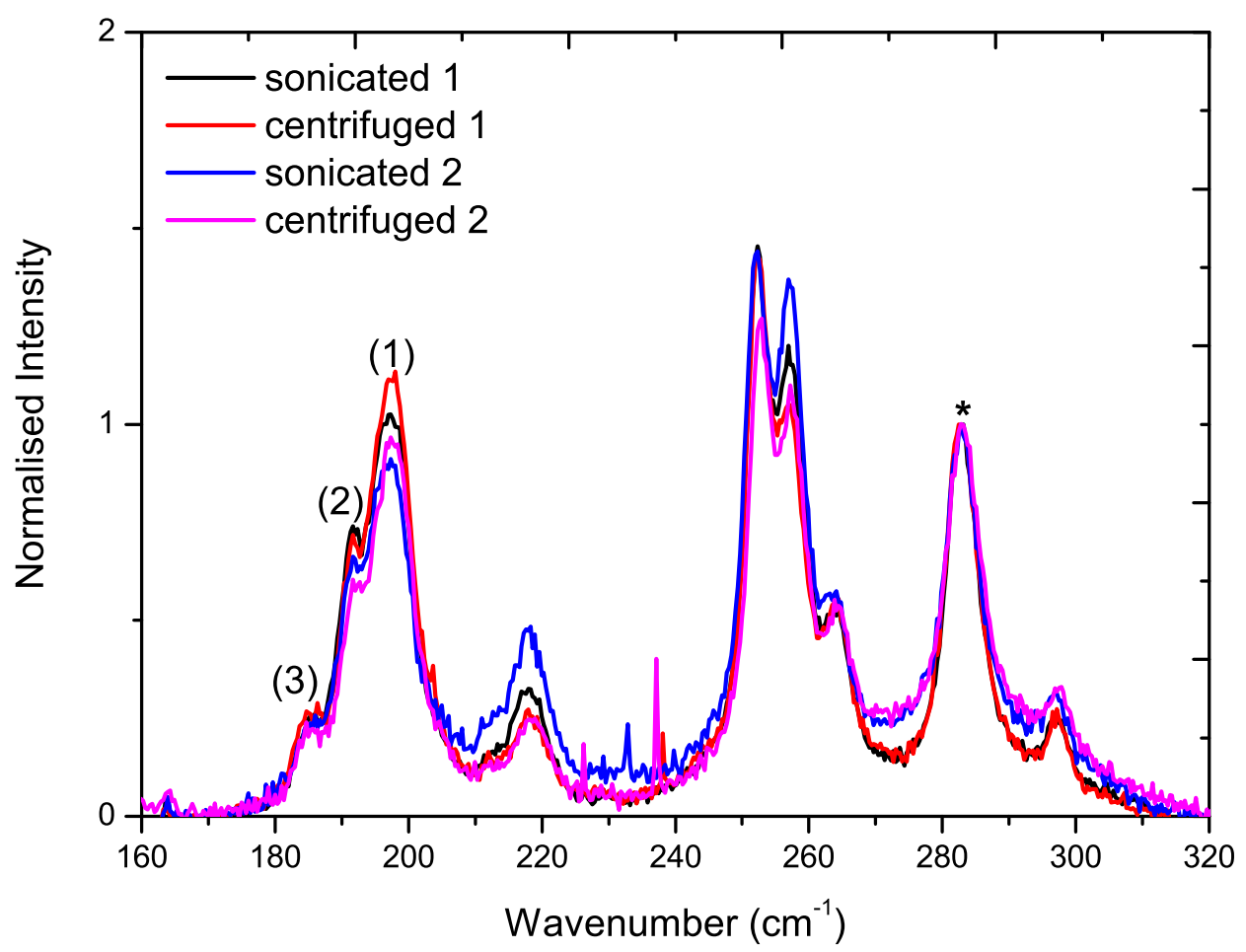

Figure 5.4: Raman spectra obtained with the $632.8 \mathrm{~nm}$ laser line of sonicated and centrifuged solutions from two different batches.

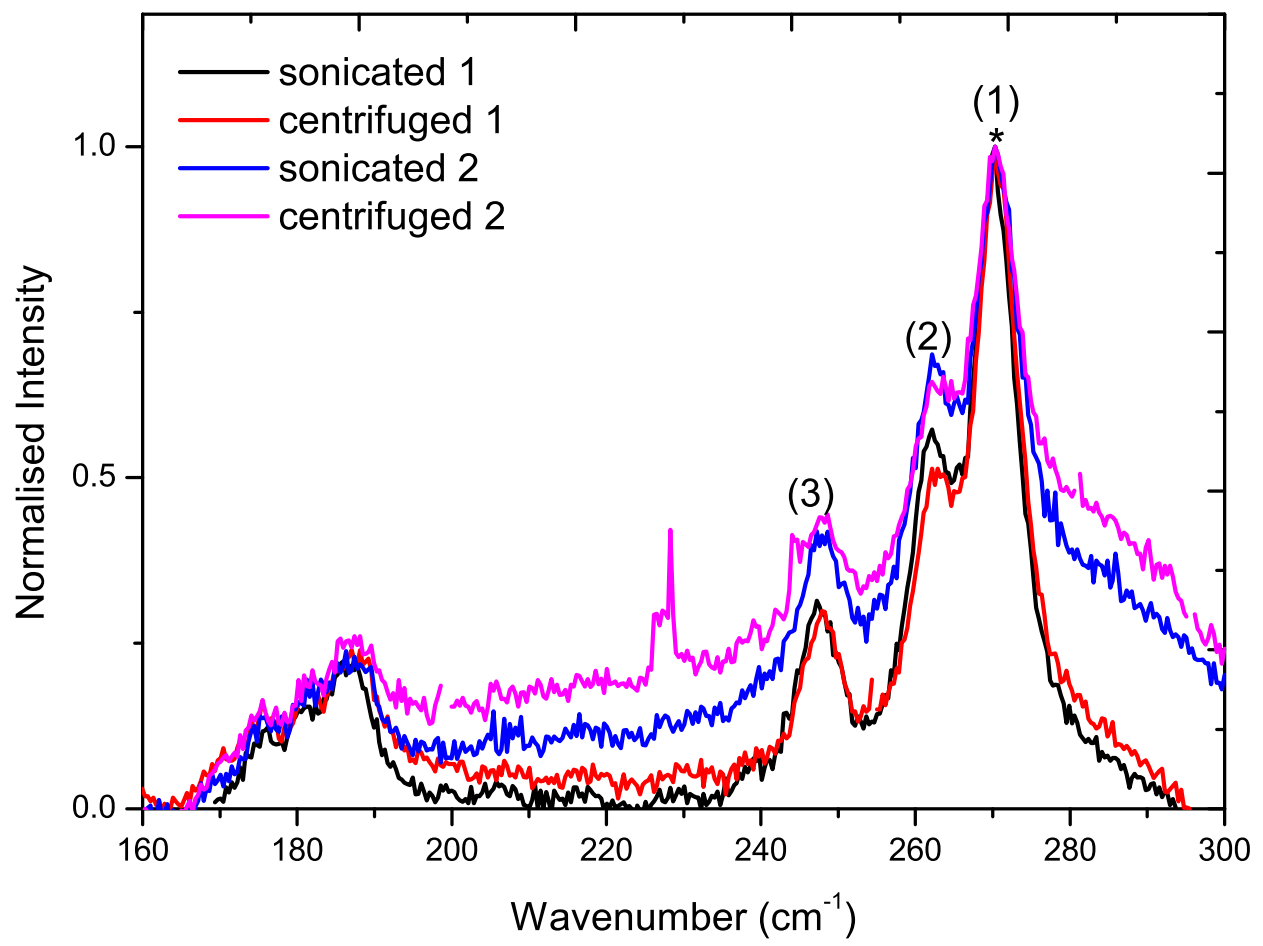

Figure 5.5: Raman spectra obtained with the $514.5 \mathrm{~nm}$ laser line of sonicated and centrifuged solutions from two different batches. The broad peak at $\sim 280$ $\mathrm{cm}^{-1}$ is from the butylamine. 

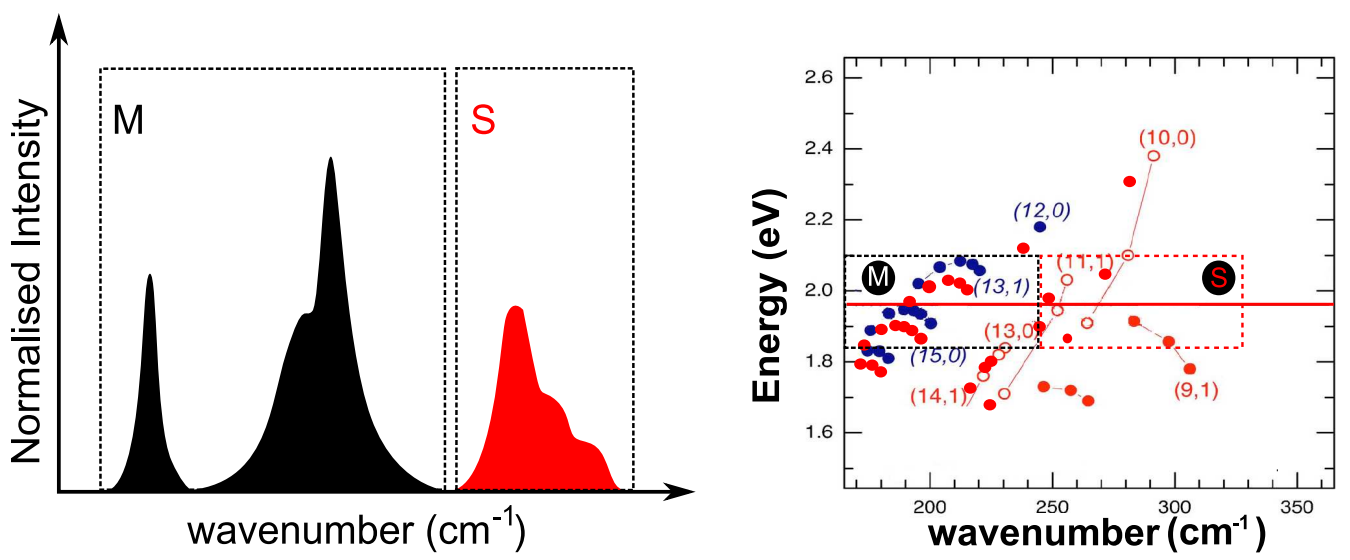

Figure 5.6: Division by electronic type of a possible RBM spectrum under the $632.8 \mathrm{~nm}$ laser. $\mathrm{M}$ and $\mathrm{S}$ represent the RBM contribution from metallic and semiconducting tubes respectively.

energy $632.8 \mathrm{~nm}$ laser.

The relative RBM signal can be used as a qualitative comparison of selectivity between treated and untreated samples [114, 98]. However, calculating the enrichment factor from this parameter is not trivial. The ratio of metallic to semiconducting tubes can be obtained by comparing the integrated area under each peak $[16,18,115]$. Figure 5.6 shows the RBM spectrum divided by the contribution of each electronic type. The signal fraction due to a given electronic type is calculated by dividing the integrated area by the integrated area of all the RBMs. For example, the metallic signal fraction in Figure 5.6 would be found using the simple formula: Area(black)/ Area(black+red).

The convoluted RBM peaks observed in the spectra are a combination of peaks corresponding to an individual nanotube. The individual RBMs were deconvoluted from the observed composite peaks using QRAP (Quick Raman Analysis Package) written by Eric Le Ru at Victoria University (see Figure 5.7). QRAP allows the user to control or vary the peak frequency, intensity, linewidth and linear background. It fits a linear background tailored to the extreme points of 


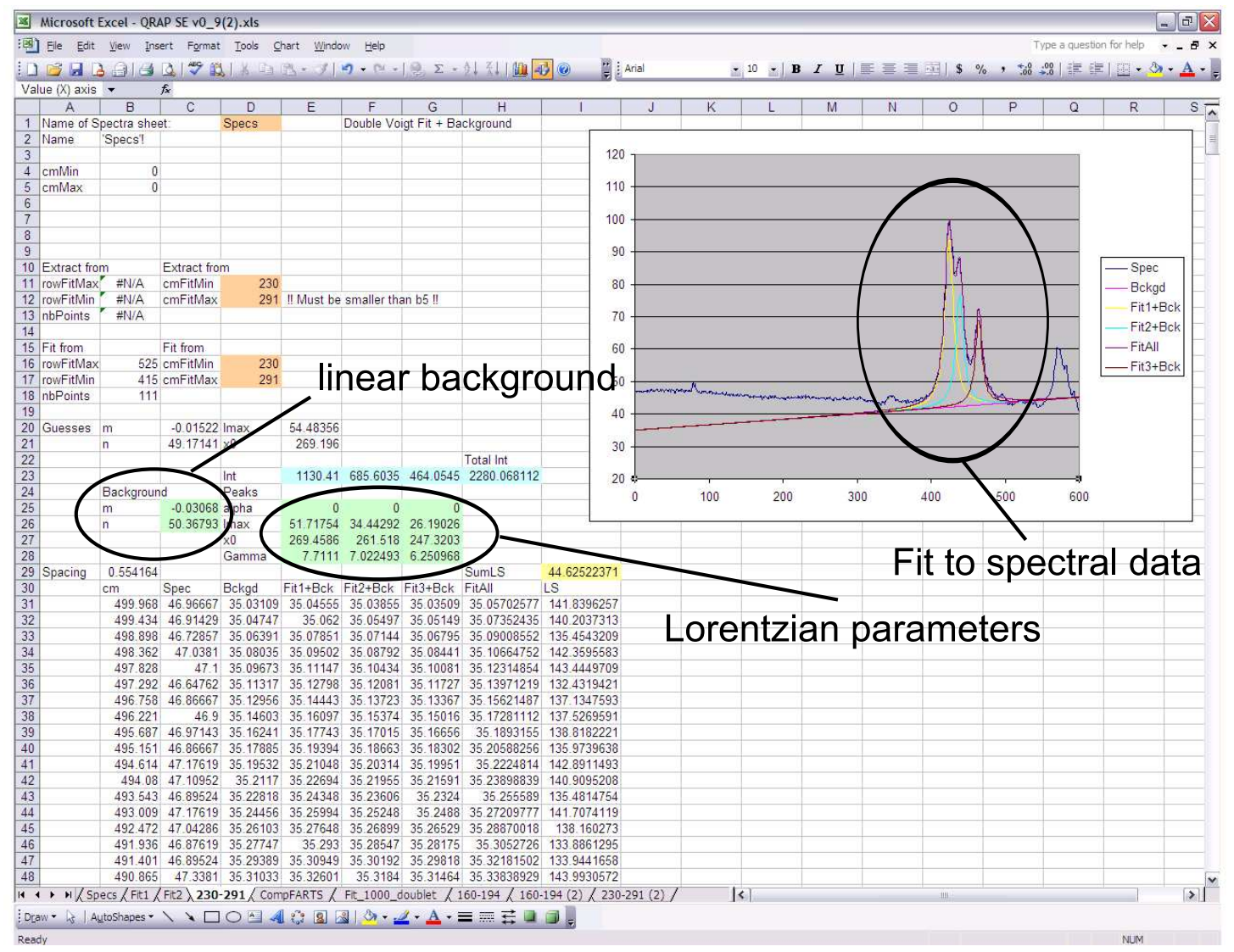

Figure 5.7: Labelled screenshot of a QRAP fitting session.

the chosen composite peak which can then be deconvoluted using Lorentzians as singlet, doublet or triplet. Initial values for the individual RBM frequencies were selected after consulting the assignments of Maultzsch et al. [49]. Once initial values for the peaks were chosen, a least squares fitting session was carried out to establish the unconstrained parameters. The output from the least squares fit also gave the integrated area under each deconvoluted peak. These peak areas were then summed to give both the integrated area for all the observed RBMs and the contribution from each electronic type. The evaluation was only carried out for the spectra from the $632.8 \mathrm{~nm}$ excitation where peaks observed below 245 $\mathrm{cm}^{-1}$ were assigned to metallic tubes and peaks above this value were assigned to semiconducting tubes [49]. Data from the $514.5 \mathrm{~nm}$ laser line was not used due to non-removable contributions from the solvent and surfactant. 


\begin{tabular}{|l|l|l|}
\hline Solution & Metallic & Semiconducting \\
\hline \hline Sonicated 1 & 0.40 & 0.60 \\
\hline Sonicated 2 & 0.37 & 0.63 \\
\hline Centrifuged 1 & 0.41 & 0.59 \\
\hline Centrifuged 2 & 0.40 & 0.60 \\
\hline Raw powder & 0.33 & 0.67 \\
\hline \hline
\end{tabular}

Table 5.1: The relative fractions of metallic and semiconducting tubes in solution and powder under the $632.8 \mathrm{~nm}$ laser.

Table 5.1 shows the fraction of metallic and semiconducting tubes probed by the red laser in both the sonicated and centrifuged dispersions as well as the raw powder. The average fraction of metallic to semiconducting tubes was calculated to be $0.4 / 0.6$ or a ratio of $2: 3$ for both the sonicated and centrifuged solutions. This result contradicts previously reported metallic enrichment of 87 $\%$ in amine/THF dispersions $[18,20]$.

On the other hand, the relative ratio for the powder is the predicted ratio of 1:2 which agrees with experimental studies performed on as-produced HiPCO SWNTs [116]. The minor disparity in the ratio between the solid and solution samples can be explained by the change in intensity of the metallic peaks in dispersed solutions due to phonon-coupling effects. While a comparison between dispersed solutions accounts for this enhancement, the difference between the raw powder and solution does not. We refer to Samsonidze et al. who advised caution when comparing two different samples [16]. Hence, our conclusions are based solely on the results obtained from the solutions which indicate a non-selective process.

If we assume that the ID model discussed earlier is accurate then the semiconducting tubes in the sample would have to be mildly p-doped such that the amine can no longer discriminate by electronic type. Given that the SWNTs are 
exposed to air while preparing the dispersion, oxygen adsorption can lead to mild p-doping $[108,117]$ making the case for a non-selective process quite probable.

\subsubsection{Unbundling effects}

The observed differences in the RBMs between the raw powder and amine solution cannot be resolved by considering surfactant selectivity. The extra peaks visible in the Raman spectra taken from the powder and sample solution are still unaccounted for. So, we now consider the possibility of bundling-induced differences in the RBMs.

As described in Section 2.2, the $\mathrm{E}_{i i}$ transitions are downshifted and broadened in bundled samples. The latter effect implies that nanotubes that were far from the resonance conditions in a less bundled sample now drift into resonance. Hence, peaks that only show up in aggregated samples are expected to have a larger energy difference between their $\mathrm{E}_{i i}$ transition and the laser energy. The minimum and maximum values of this energy difference can be used to estimate a resonance window for each sample.

To study the effects of unbundling, the Raman spectrum of a solid sample of raw HiPCO powder was compared with a solution sample of 7:1 THF:butylamine dispersion. RBM spectra were obtained from five different spots on the powder sample and two batches of centrifuged solutions. Quantitative analyses were performed solely on the spectra taken using the $632.8 \mathrm{~nm}$ laser. The spectra collected using the $514.5 \mathrm{~nm}$ excitation are presented for completeness.

QRAP fits were performed on spectra calibrated using a measured silicon

peak at $520 \mathrm{~cm}^{-1}$. This consistent point of reference ensured that RBMs from 
different samples could be compared with each other. Linewidths of 4 or $5 \mathrm{~cm}^{-1}$ were found to yield good fits in the majority of cases. This value is close to the natural linewidth of an individual nanotube which was independently found to be $\sim 3 \mathrm{~cm}^{-1}$ by both Iliev et al. [118] and Jorio et al. [119]. We required a slightly larger value since $3 \mathrm{~cm}^{-1}$ was too narrow to fit our spectra. This is not unexpected since linewidths up to $8 \mathrm{~cm}^{-1}$ can occur due to instrumental broadening, defects, interactions with other tubes etc. [119].

Laser lines (e.g. $514.5 \mathrm{~nm}$ line) that resonantly excite a small number of tubes often show 'bundling' peaks in the spectra of aggregate samples. When the exact assignments are not known, these peaks can also be used as visual markers to qualitatively assign the extent of bundling. The red circles shown in Figure 5.1 indicate the 'bundling' peaks in the $200-240 \mathrm{~cm}^{-1}$ region for the powder under the $514.5 \mathrm{~nm}$ laser. The resonance shift is not given for these peaks - corresponding to the $\mathrm{E}_{33}^{S}$ transitions of large diameter SWNTs - since there are no experimentally established literature values available. However, we can assign the highlighted peaks to resonance broadening due to aggregation since bundled samples from both Murakami et al. [50] and Heller et al. [57] show these same peaks. On the other hand, well-exfoliated SDS-SWNT dispersions show either the metallic peaks [58] or both the metallic peaks and the semiconducting peaks under $200 \mathrm{~cm}^{-1}[50,57]$. Our centrifuged butylamine-SWNT dispersions only show the metallic triplet above $240 \mathrm{~cm}^{-1}$ and the semiconducting triplet below $200 \mathrm{~cm}^{-1}$ which indicate that these dispersions are unbundled to some extent.

In Figure 5.2 the two 'bundling' peaks observed only in the powder are marked with red circles. Since the $\mathrm{E}_{i i}$ transitions are well established for the tubes resonant with the $632.8 \mathrm{~nm}$ laser, we can quantify the resonance window. The energy shift from the laser for the resonant nanotubes in both the powder and the solu- 


\begin{tabular}{|l|l|l|}
\hline \multicolumn{2}{|c|}{$\mathbf{6 3 2 . 8} \mathbf{~ n m ~ l a s e r ~}$} \\
\hline \hline Powder $\left(\mathbf{c m}^{-1}\right)$ & Solution $\left(\mathbf{c m}^{-1}\right)$ & $\mathrm{E}_{\text {laser }}-\mathrm{E}_{\text {ii }}[\mathbf{4 9}](\mathbf{m e V})$ \\
\hline- & 184.8 & 24 \\
\hline $\mathbf{2 1 0 . 5}$ & - & -124 \\
\hline- & 197.2 & 26 \\
\hline 214.2 & 217.4 & -115 \\
\hline 217.2 & 219.3 & -97 \\
\hline $\mathbf{2 4 1 . 3}$ & - & -220 \\
\hline 248.6 & 252.6 & 15 \\
\hline 254.7 & 257.5 & -71 \\
\hline 280.6 & 283.1 & 45 \\
\hline- & 297.1 & 103 \\
\hline \hline
\end{tabular}

Table 5.2: This table shows shifts in the $\mathrm{E}_{i i}$ transitions between our powder and butylamine-SWNT dispersions, for RBMs assigned to an unambiguous chiral index assignment using data from Maultzsch et al. [49]. Only the tubes visible under the $632.8 \mathrm{~nm}$ laser excitation are given here.
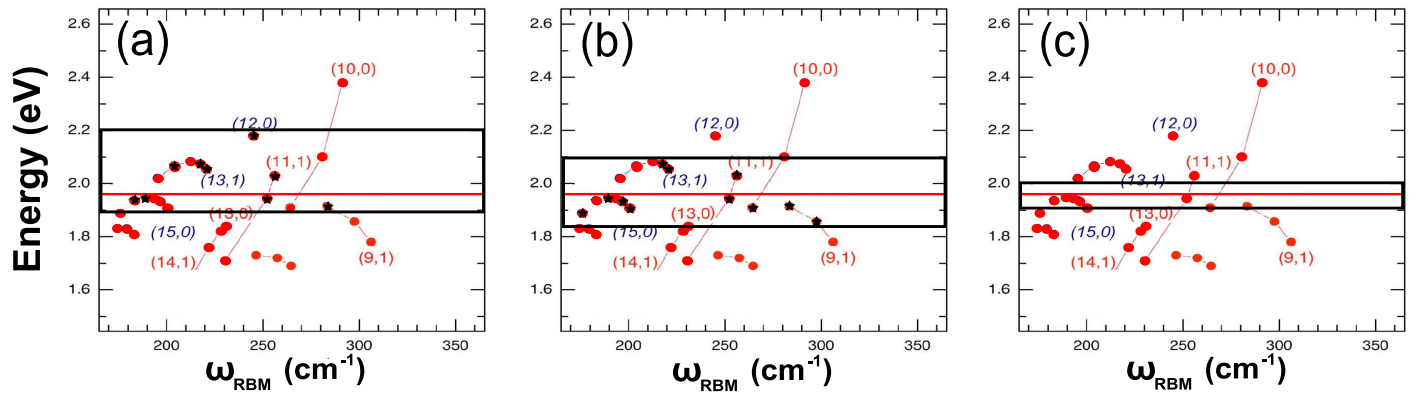

Figure 5.8: Three Kataura plots that show the resonance window in samples with different levels of aggregation. (a) Resonance window for the raw HiPCO powder. (b) Resonance window for centrifuged 7:1 butylamine dispersions. (c) Resonance window for a dispersion comprised of individual nanotubes wrapped in SDS. The RBMs observed in the powder (a) and solution (b) are marked with a black star. The Kataura plots were modified from [49].

tion are calculated from experimentally determined $\mathrm{E}_{i i}$ transitions of Maultzsch et al. [49]. Assignments of Maultzsch et al. were obtained from a solution of HiPCO SWNTs dispersed using SDS in $\mathrm{D}_{2} \mathrm{O}$.

The results are tabulated in Table 5.2 with the bundling peaks depicted in boldface. The apparent resonance windows for the powder and solution and are presented visually in Figure 5.8. 
Figure 5.8 shows how resonance broadening leads to an estimate of the resonance window. The resonance windows are either symmetrical (Figure 5.8 (c)) or asymmetrical (Figure $5.8(\mathrm{a}, \mathrm{b})$ ) about the laser line. The asymmetry is not unusual as it was also observed in a similar Raman study of aggregated samples conducted by Ericson et al. [58]. Heller et al. [57] experimentally deduced that resonant nanotubes in fully-exfoliated dispersions fall within $40 \mathrm{meV}$ of the laser excitation (Figure $5.8(\mathrm{c})$ ). The window broadens if the sample probed by the laser exhibits some degree of bundling like our butylamine dispersions whose window is depicted in Figure 5.8 (b). For a heavily aggregated sample such as the raw powder, the resonance window expands even further (Figure 5.8 (a)).

The tubes picked out by the $632.8 \mathrm{~nm}$ laser fall within $115 \mathrm{meV}$ of the laser line for butylamine dispersions and $220 \mathrm{meV}$ for the raw powder. Resonance windows up to $200 \mathrm{meV}$ were measured by Ericson et al. in bundled samples [58] which agrees with the maximum energy shift for the raw powder. The narrower window of $115 \mathrm{meV}$ indicates that the butylamine dispersions are comprised of smaller bundles than the powder.

Figures 5.4 and 5.5 show certain metallic peaks with a consistent pattern in the intensity under both lasers (labelled 1 - 3 in brackets). Assigning these peaks reveals a pattern of increasing intensity with decreasing diameter and chiral angle (refer to Table 5.3). This same trend was also observed by Maultzsch et al. who attributed it to an enhanced phonon coupling with decreasing diameter and chiral angle [49]. This effect is only observed in metallic tubes since they are evenly clustered about the laser excitation. From Table 5.3, we see that the metallic tubes lie within $60 \mathrm{meV}$ and $52 \mathrm{meV}$ for the $514.5 \mathrm{~nm}$ and $632.8 \mathrm{~nm}$ lasers respectively. In Figure 5.5, we see that this pattern is broken since the broadening 


\begin{tabular}{|c|c|c|c|c|}
\hline \multicolumn{5}{|l|}{$632.8 \mathrm{~nm}$ Laser } \\
\hline Frequency $\left(\mathrm{cm}^{-1}\right)$ & Type & $\mathrm{d}(\AA)$ & $\theta_{C}\left(^{\circ}\right)$ & 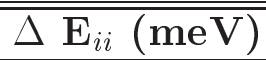 \\
\hline $183.2^{\dagger}(3)$ & $\mathrm{M}$ & 12.94 & 24.8 & 24 \\
\hline $189.4^{\dagger}(2)$ & M & 12.44 & 19.1 & 12 \\
\hline $196.3^{\dagger}(1)$ & $\mathrm{M}$ & 11.83 & 6.6 & 26 \\
\hline $200.4^{\dagger}(1)$ & $\mathrm{M}$ & 11.75 & 0 & 52 \\
\hline $252.1^{\dagger}$ & $\mathrm{S}$ & 9.24 & 12.7 & 15 \\
\hline $256.0^{\dagger}$ & $\mathrm{S}$ & 9.03 & 4.3 & -71 \\
\hline $264.2^{\dagger}$ & $\mathrm{S}$ & 8.83 & 27.5 & 51 \\
\hline $283.3^{\dagger}$ & $\mathrm{S}$ & 8.18 & 24.5 & 45 \\
\hline $297.5^{\dagger}$ & $\mathrm{S}$ & 7.72 & 15.3 & 103 \\
\hline \multicolumn{5}{|l|}{$514.5 \mathrm{~nm}$ Laser } \\
\hline Frequency $\left(\mathrm{cm}^{-1}\right)$ & Type & $\mathrm{d}(\AA)$ & 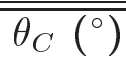 & $\Delta \mathbf{E}_{i i}(\mathbf{m e V})$ \\
\hline $175.8^{\ddagger}$ & $\mathrm{S}$ & 13.84 & 23.41 & $\mathrm{NA}$ \\
\hline $181.7^{\ddagger}$ & $\mathrm{S}$ & 13.36 & 17.99 & $\mathrm{NA}$ \\
\hline $186.3^{\ddagger}$ & $\overline{\mathrm{S}}$ & 13.0 & 12.2 & $\mathrm{NA}$ \\
\hline $247.8^{\dagger}(3)$ & $\mathrm{M}$ & 9.50 & 30 & 40 \\
\hline $262.7^{\dagger}(2)$ & $\mathrm{M}$ & 8.90 & 22.4 & 60 \\
\hline $272.7^{\dagger}(1)$ & $\mathrm{M}$ & 8.47 & 13.9 & 20 \\
\hline
\end{tabular}

Table 5.3: Details of the predominant RBMs observed under the $632.8 \mathrm{~nm}$ and $514.5 \mathrm{~nm}$ laser lines. The frequencies shown in green and red have intensities inversely proportional to diameter and chiral angle. The numbers in brackets represent the ranking by relative intensity of the given peak within the triplet. Frequencies marked with ${ }^{\dagger}$ were sourced from Maultzsch et al. [49]. $\mathrm{E}_{33}^{S}$ semiconducting transitions marked with ${ }^{\ddagger}$ were obtained from Wiltshire et al. [120].

and shifting of the $\mathrm{E}_{i i}$ transitions moves the metallic tubes away from their close proximity to the laser energy. This behaviour indicates that most of the solvated tubes exist in bundles that are too small to affect the electronic transition energies.

Dispersions prepared with different THF:butylamine ratios (see Table 5.4 for details) are alike regardless of the concentration of dispersed nanotubes. Figures 5.9 and 5.10 shows the RBM spectra for four dispersions of varying THF:amine ratios collected in solution under the $632.8 \mathrm{~nm}$ and $514.5 \mathrm{~nm}$ lasers respectively.

Normalised solution spectra shown in Figures 5.9 and 5.10 are consistent in 


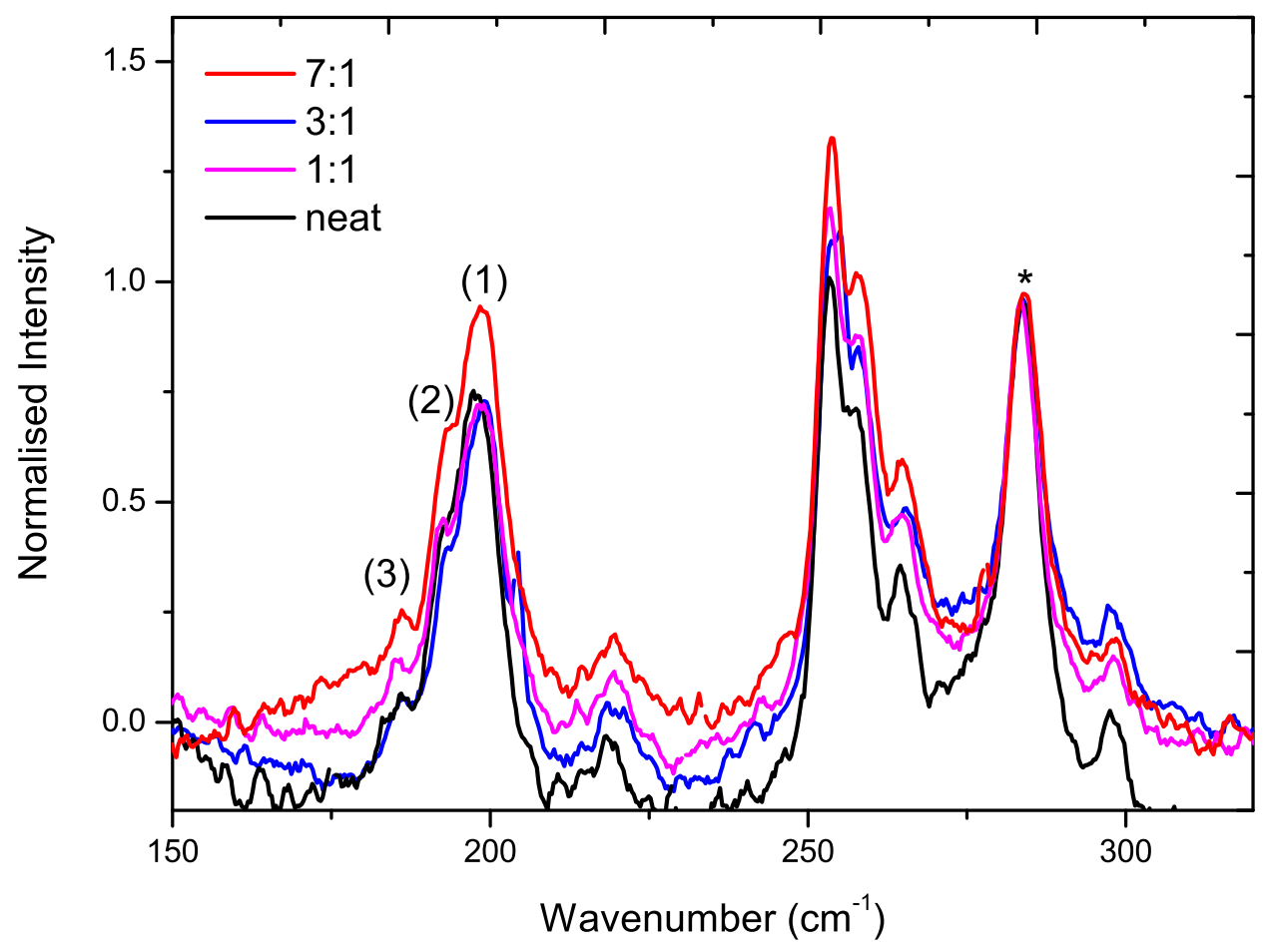

Figure 5.9: Raman spectra obtained with the $632.8 \mathrm{~nm}$ laser line for four solutions prepared with different THF:butylamine ratio.

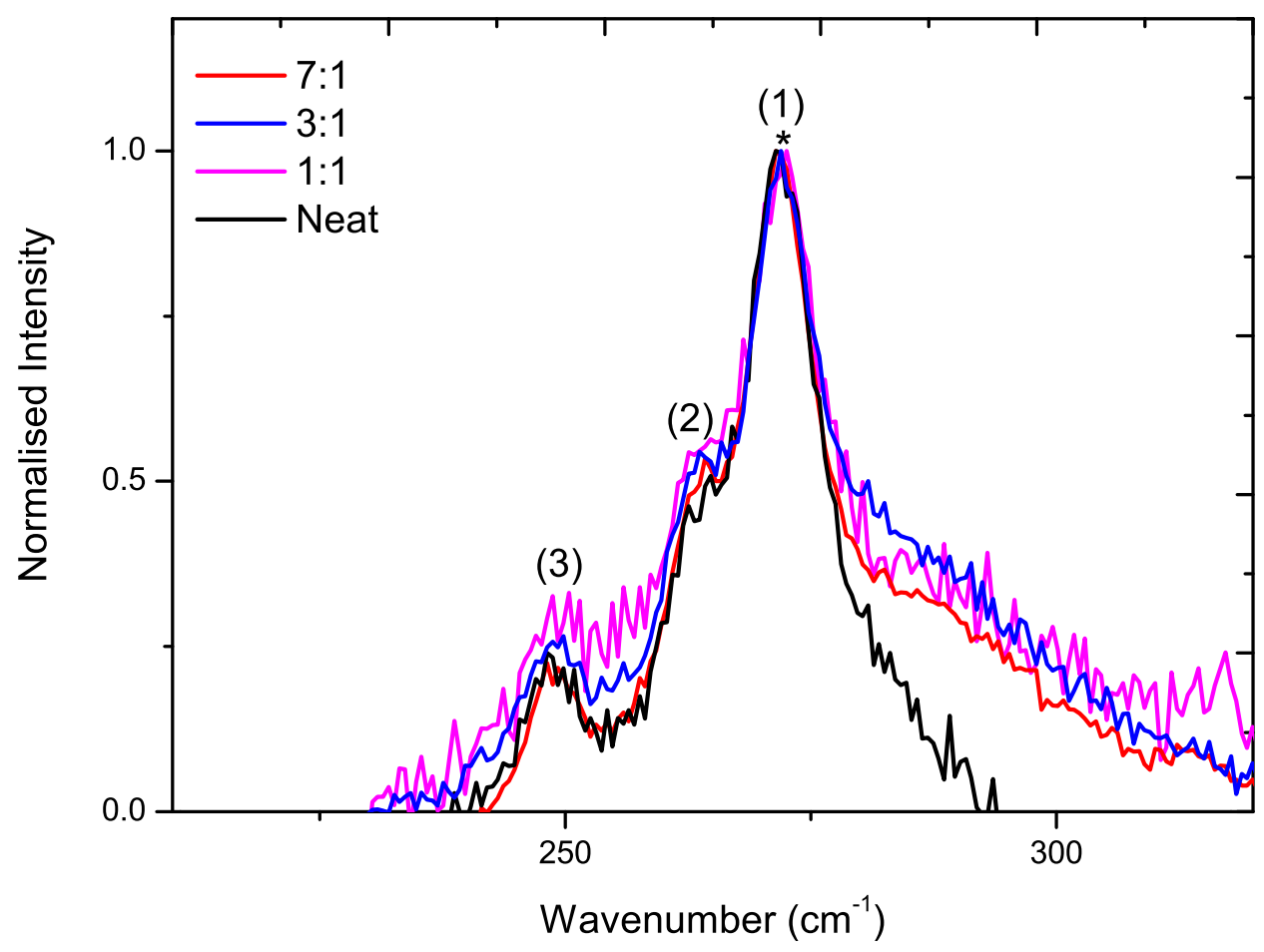

Figure 5.10: Raman spectra obtained with the $514.5 \mathrm{~nm}$ laser line for four solutions prepared with different THF:butylamine ratio. 


\begin{tabular}{|l|l|}
\hline Abbreviation & Description \\
\hline \hline $7: 1$ & $7: 1$ ratio of THF:butylamine \\
\hline $3: 1$ & $3: 1$ ratio of THF:butylamine \\
\hline $1: 1$ & $1: 1$ ratio of THF:butylamine \\
\hline Neat & dispersion prepared in butylamine \\
\hline \hline
\end{tabular}

Table 5.4: Description of the dispersions used to understand the efficacy of butylamine.

relative intensity and the peak positions. These features indicate that only a small amount of butylamine is required to effectively disperse SWNTs. This characteristic is quite useful since a reduction in the amount of residual surfactant also minimises post-deposition treatments. The SEM images in Section 4.2 show that a fair amount of butylamine is left over on the SWNT network even when a 7:1 dispersion is used. While the annealing treatment is not cumbersome, further development of our film fabrication process would require greater time efficiency. For these reasons, a smaller amount of butylamine is ideal.

\subsubsection{Raman mode shifts}

In the previous sections, the absolute value of the RBM frequencies was used to match the peaks observed by Maultzsch et al. [49] in SDS-SWNT dispersions for the purpose of chiral assignment. The values themselves were ignored. Yet, Table 5.2 shows that the RBM frequency in the centrifuged solution is upshifted from the powder. As discussed in Section 2.2.3, shifts in the RBM frequency occur due to a number of physicochemical interactions such as charge transfer, surfactant pressure and aggregation. In this section, the unbundling of nanotubes by the butylamine is shown to be responsible for the observed frequency upshift in the centrifuged solution compared to the raw powder. 


\section{RBM shifts}

The main differences between the centrifuged dispersion and the raw powder include the nanotube environment, surfactant-nanotube interactions and change in bundle size. Each of these changes have been shown to lead to an upshift in RBM frequency.

Izard et al. showed that frequency upshifts in SWNT dispersions were due to pressure from the surfactant and solvent on the SWNT sidewall [51]. The upshifts in the RBMs were found to be similar to those induced by hydrostatic pressure $[52,53,56]$. However, Maultzsch et al. attributed shifts in frequency to charge transfer between the nanotube and surfactant [49]. Within this framework, upshifts in the RBM frequency were attributed to a shortening of the $\mathrm{C}-\mathrm{C}$ bond due to charge donation from the nanotube to the surfactant. This mechanism is supported by Raman studies of doped nanotubes where upshifts were shown to occur in p-doped nanotubes [54, 55]. Lastly, Murakami et al. reported an upshift of $\sim 3 \mathrm{~cm}^{-1}$ in the RBM frequency of centrifuged dispersions compared to the raw powder [50].

In order to elucidate the mechanism or combination of mechanisms responsible for shifts in the RBM frequency, a series of comparisons were made between solid and solution samples (given in Table 5.5). The results presented in this chapter were obtained by using a fine spectrometer grating (1800 lines/mm) which enabled a spectrometer resolution of $0.3 \mathrm{~cm}^{-1}$. As mentioned in Section 5.1.2, all the Raman spectra were corrected to the silicon peak at $520 \mathrm{~cm}^{-1}$. Only those RBMs that could be assigned a unique chiral index from Maultzsch et al. [49] without any ambiguity were used to calculate the frequency shift.

RBM peaks for all the samples listed in Table 5.5 were deconvoluted using 


\begin{tabular}{|l|l|}
\hline Sample & Spectra \\
\hline \hline raw powder & 5 \\
\hline centrifuged solution & 2 \\
\hline precipitate & 2 \\
\hline unannealed film & 5 \\
\hline \hline
\end{tabular}

Table 5.5: Sample description and the number of spectra used to obtain an average for each RBM peak.

QRAP. The measured frequency was then averaged over the given number of spectra. For example, each RBM peak from the powder sample was an average of five different values and the uncertainty of this average was the calculated standard error. The frequency shift between the compared samples was calculated from peaks of unambiguous assignment. The propagated errors for each RBM are shown by the error bars in Figures $5.12-5.14$ and 5.22. The average frequency shift between two samples was calculated from the absolute value of all the RBM shifts. For example, the average shift between the powder and centrifuged butylamine dispersion was calculated from five values. The standard error of the average shift was taken as the uncertainty. The average shift and uncertainty are depicted in Figures 5.11, 5.12, 5.13, 5.14 and 5.22 as the solid and dotted lines respectively.

First, the RBM frequencies of dispersed nanotubes were compared to that of raw powder. An upshift of $3.0 \pm 0.2 \mathrm{~cm}^{-1}$ was observed between the centrifuged butylamine dispersion and raw powder (Figure 5.11). A similar shift $(3.3 \pm 0.1$ $\mathrm{cm}^{-1}$ ) is also seen between the SDS dispersion of Maultzsch et al. [49] and our raw powder (Figure 5.12). The frequency shift between the SDS and butylamine dispersions was calculated to be $0.4 \pm 0.2 \mathrm{~cm}^{-1}$. Thus, the observed upshift between the dispersions and the powder could be due to unbundling, charge transfer or surfactant pressure. 


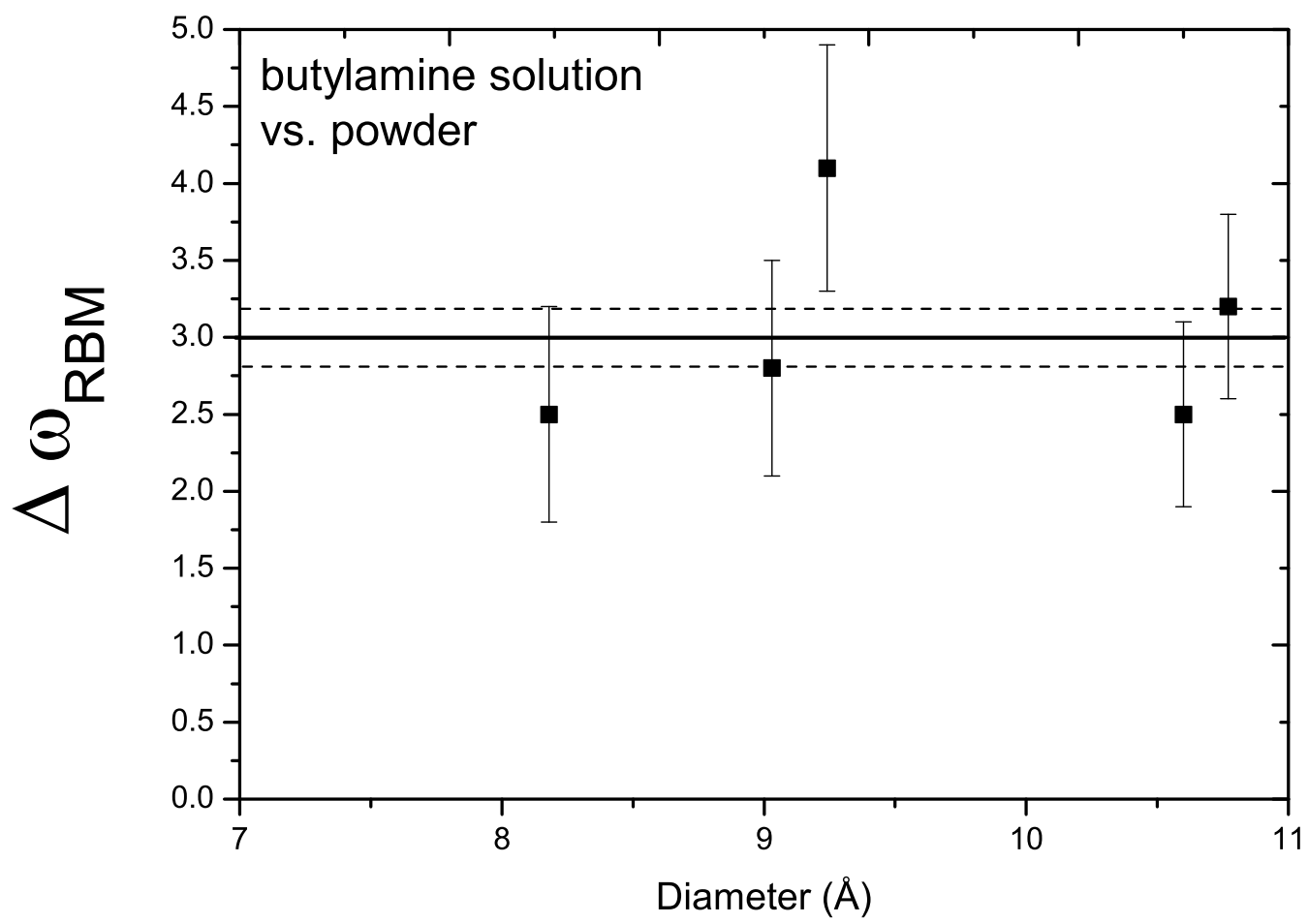

Figure 5.11: RBM shifts between the centrifuged amine dispersion and the raw powder. The solid black line indicates the average shift while the dotted line indicates the error margin.

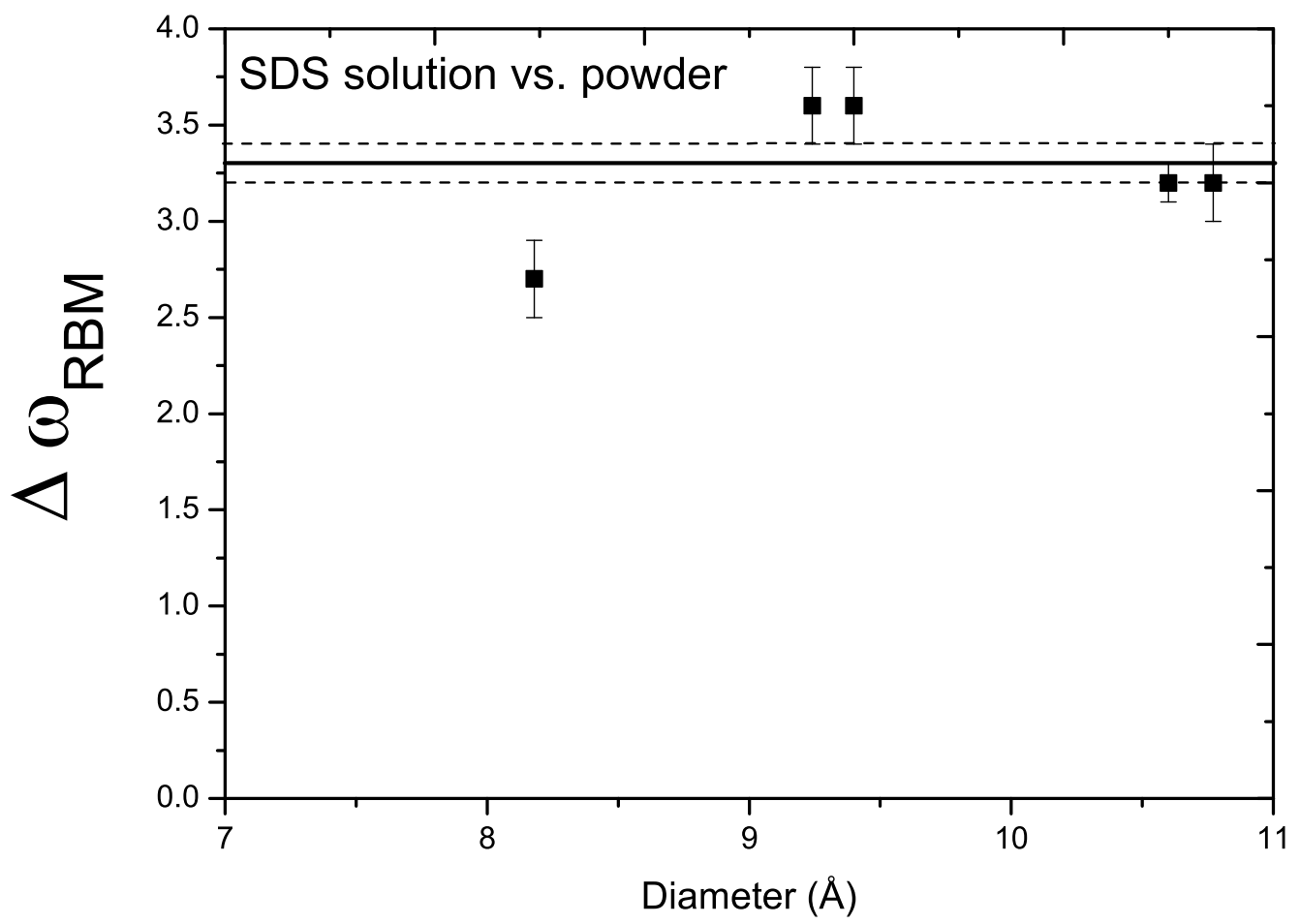

Figure 5.12: RBM shifts between the SDS dispersion of Maultzsch et al. [49] and the raw powder. The solid black line indicates the average shift while the dotted line indicates the error margin. 


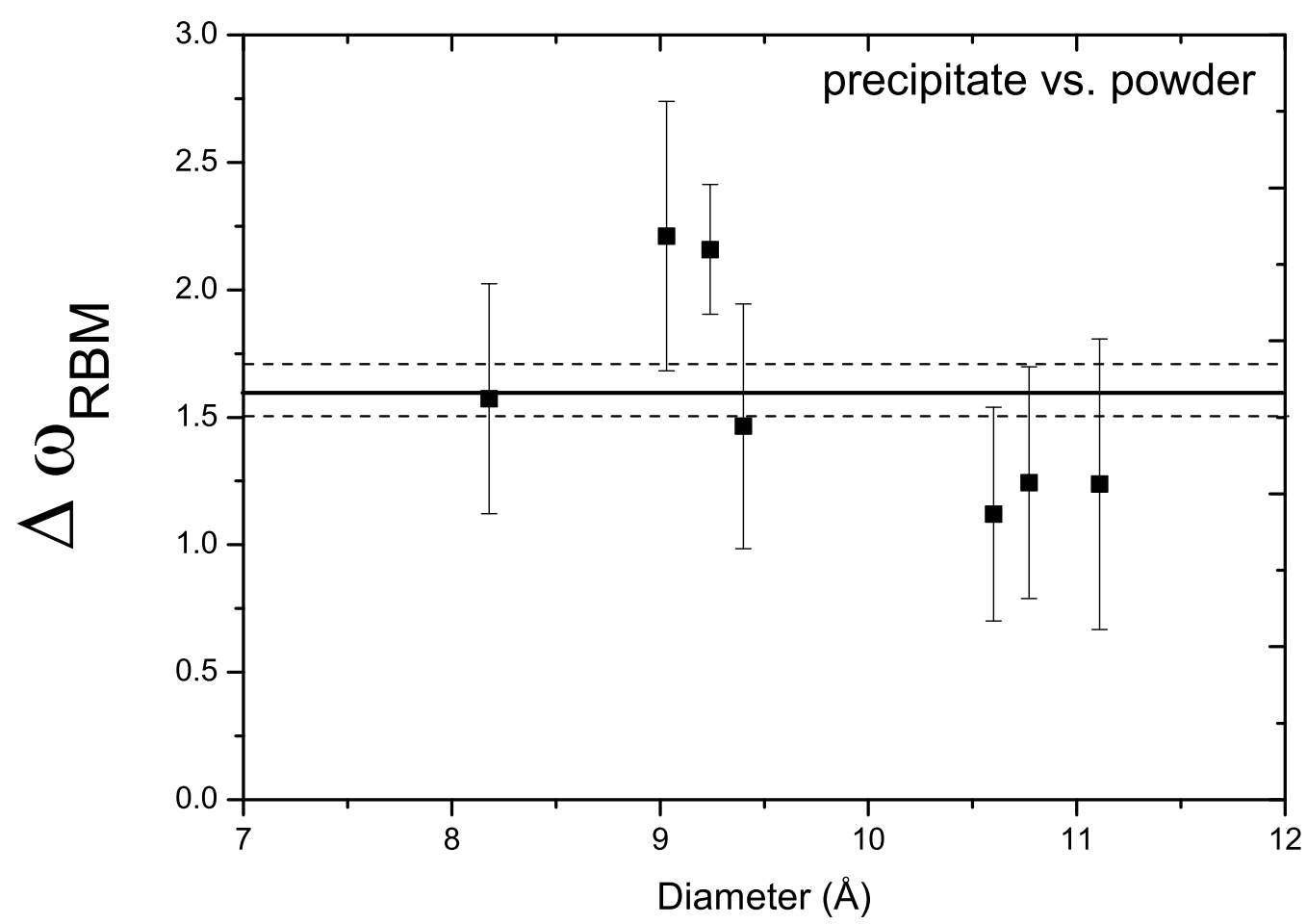

Figure 5.13: RBM shifts between the precipitate collected after centrifugation and the raw powder. The solid black line indicates the average shift while the dotted line indicates the error margin.

Izard et al. calculated the surfactant pressure from the CED of the surfactant [51]. A similar calculation was performed for our butylamine dispersions. The CED was obtained from the Hildebrand parameter [105] - the square root of the CED - giving 338.56 MPa and 345.96 MPa for butylamine and THF respectively. A RBM shift of $2.9 \pm 0.5 \mathrm{~cm}^{-1}$ was calculated for both solvents from the relation summarised by Izard et al. relating RBM shift to the surfactant pressure [51] (see Section 2.2.3). The calculated shift matches our experimental shifts in solution.

Another set of comparisons is helpful at this point. This time, the raw powder was compared to the precipitate from the centrifuged dispersions. The point of comparison being that the precipitate still contains some amine though it is a solid sample. Thus, a non-negligible RBM shift implies a cause other than the surfactant pressure. The RBM shift between the precipitate and raw powder is 


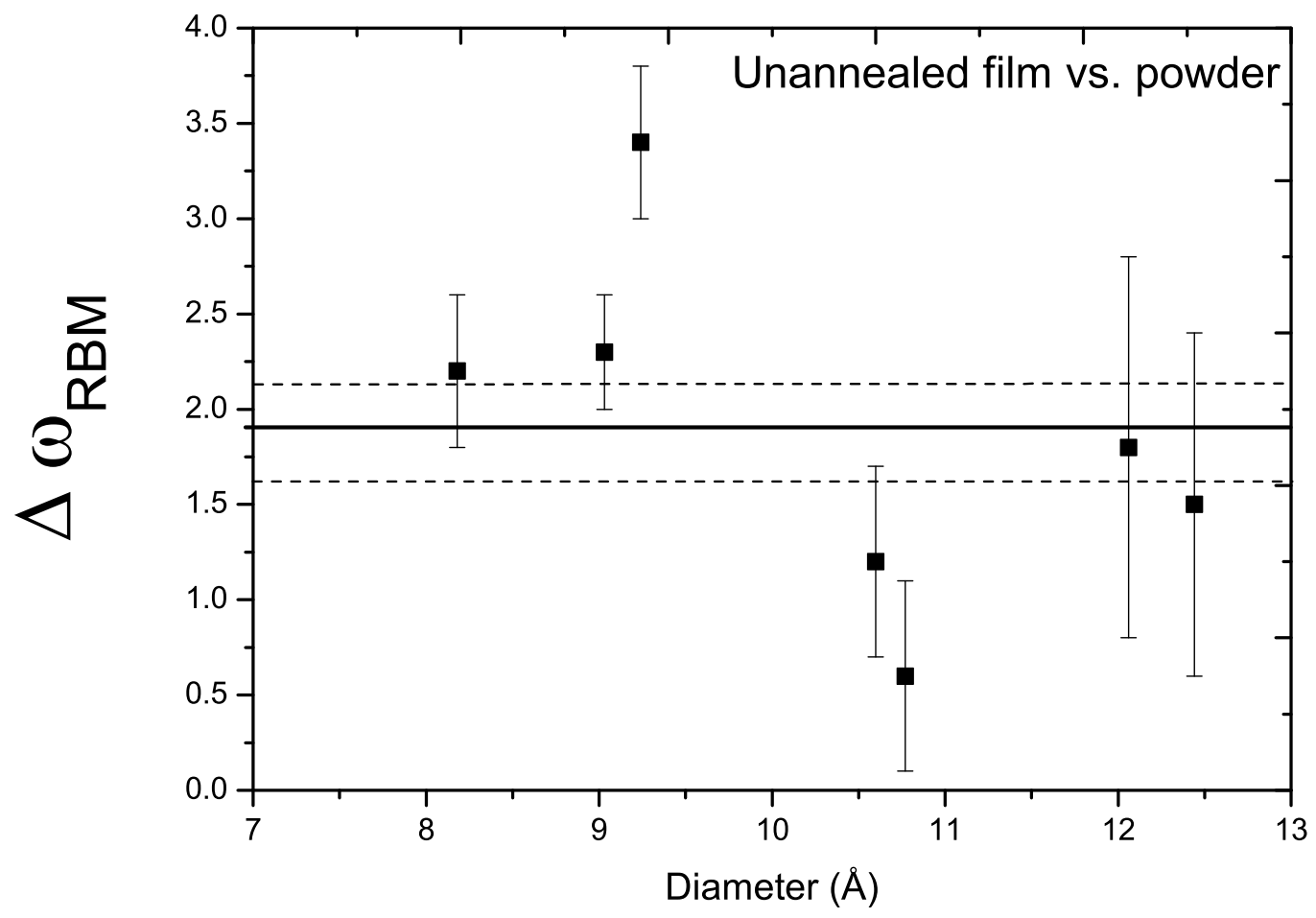

Figure 5.14: RBM shifts between an unannealed film and raw powder. The solid black line indicates the average shift while the dotted line indicates the error margin.

shown in Figure 5.13. The average shift was calculated to be $1.6 \pm 0.1 \mathrm{~cm}^{-1}$. A similar comparison between the raw powder and an unannealed SWNT thin film (Figure 5.14) yielded an upshift of $1.9 \pm 0.3 \mathrm{~cm}^{-1}$. There is little difference between the precipitate and the unannealed network (the average shift is $0.1 \pm$ $\left.0.1 \mathrm{~cm}^{-1}\right)$. Thus, we can conclude that surfactant pressure does not cause the observed RBM shift between the powder and solution.

The charge transfer theory can also be dismissed at this point. An upshift upon amination would indicate that butylamine acts as an acceptor, which is an unlikely scenario since it is an electron donor. Bundles in aminated solid samples are smaller than the raw powder since the butylamine remains bound to nanotube wall. These bundles are larger than those in solution due to evaporation of butylamine during deposition of the solid sample. Together, these observations 
imply unbundling by the butylamine rather than charge transfer as the cause for the RBM shift.

While we can conclude that unbundling causes an upshift in RBM frequency in our samples, the reasons for the observed upshift are not known. Theoretical calculations by Henrard et al. predicted a significant downshift upon bundling [45] which disagrees with our results. Murakami et al. [50] are the only other group who report a similar upshift $\left(2-3 \mathrm{~cm}^{-1}\right)$ between raw powder and a SWNT-SDS dispersion. The narrowing of the $\mathrm{E}_{i i}$ transitions upon unbundling was implicated as the cause of the observed upshift by Murakami et al. [50]. This cause was refuted in Section 2.2.3. Instead, we speculate that C-C bonds are softened in bundled tubes since the $\pi$-electrons can delocalise over several tubes resulting in a redshifted phonon vibration.

\section{G-mode shifts}

If the $\mathrm{C}-\mathrm{C}$ bond softens in bundles the frequency of other Raman modes would also be redshifted. In this section, changes in the frequency of the G-mode are studied as a function of bundling.

By comparing the shifts in the G-mode between areas of different bundle sizes, we might quantify a shift due to aggregation. Raman spectra were obtained from fifteen different spots on a SWNT film annealed at $300{ }^{\circ} \mathrm{C}$. The sparse coverage of the annealed network (shown in Figure 4.10) enabled sampling of both higly aggregated areas and regions with small bundles.

The frequency of the modes and their linewidths were found by deconvoluting the G-band with two Lorentzians using QRAP. No shoulders or any indications of a composite peak were visible in the G-band and all attempts to fit more than 


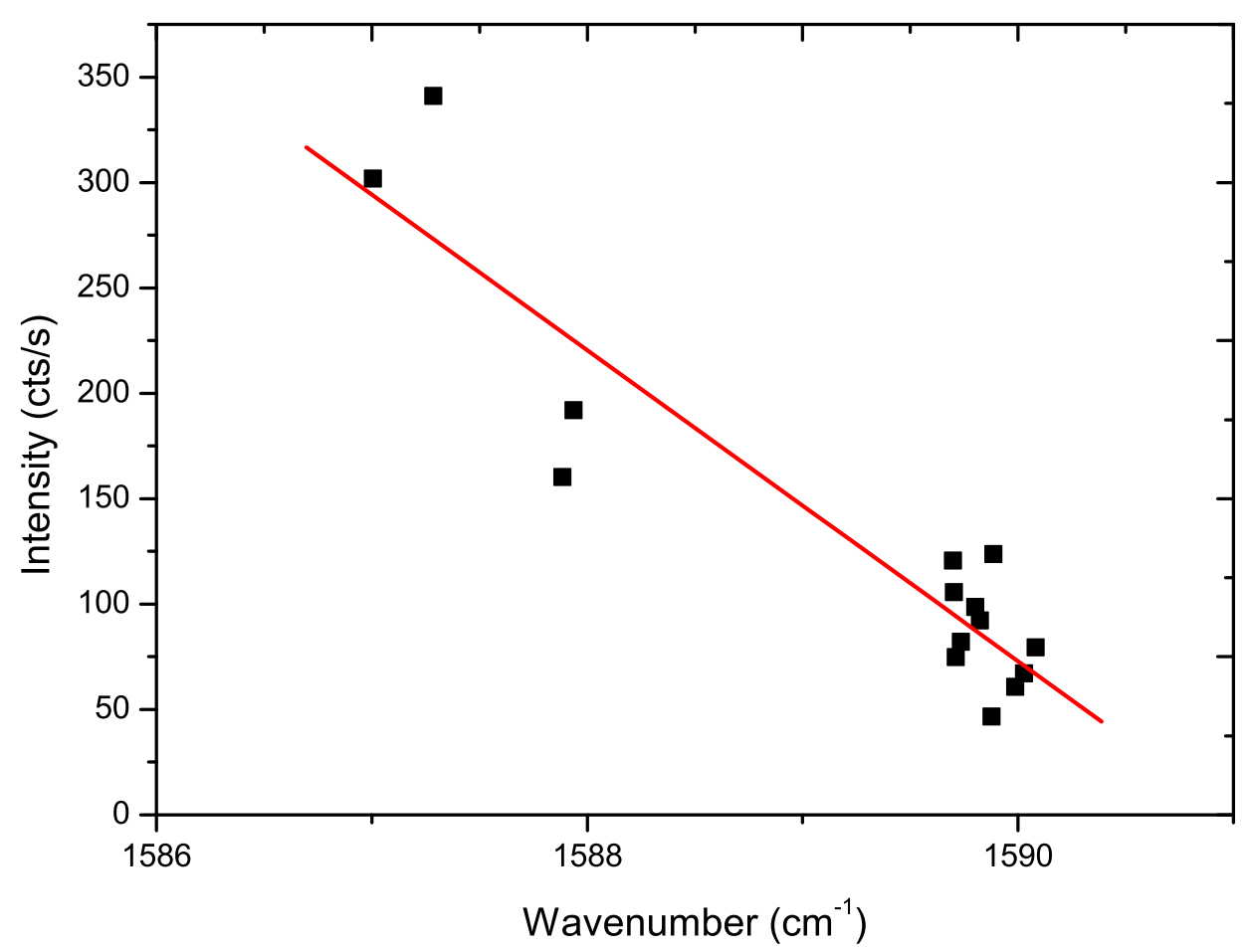

Figure 5.15: Intensity vs. frequency for the $\mathrm{G}^{+}$peak. As intensity increases, the frequency downhshifts.

one Lorentzian to each of the peaks resulted in poor fits. Our result does not agree with previous studies which found that the G-band was comprised of four to five Lorentzians in bundled samples. Only the $\mathrm{G}^{-}$mode at $1555 \mathrm{~cm}^{-1}$ of $\mathrm{E}_{2 g}$ symmetry and the mixed symmetry, $\mathrm{G}^{+}$mode at $1590 \mathrm{~cm}^{-1}$ were observed in the annealed sample. Both these modes correspond to low chiral angle tubes while modes that are absent correspond to high chiral angle tubes [39]. We speculate that annealing SWNT films to $300{ }^{\circ} \mathrm{C}$ combusts high chiral angle tubes (Section 5.2.1) which would lead to a disappearance of these high chiral angle modes.

Figure 5.15 depicts the frequency of the $\mathrm{G}^{+}$mode plotted against signal intensity. This plot shows that areas with larger clumps of nanotubes not only have a strong signal but also a redshifted $\mathrm{G}^{+}$mode. In these thin films, intensity can be related to the number of tubes sampled by the laser. This implies that aggregation induces a downshift in the dominant $\mathrm{G}^{+}$peak. Further proof of the 


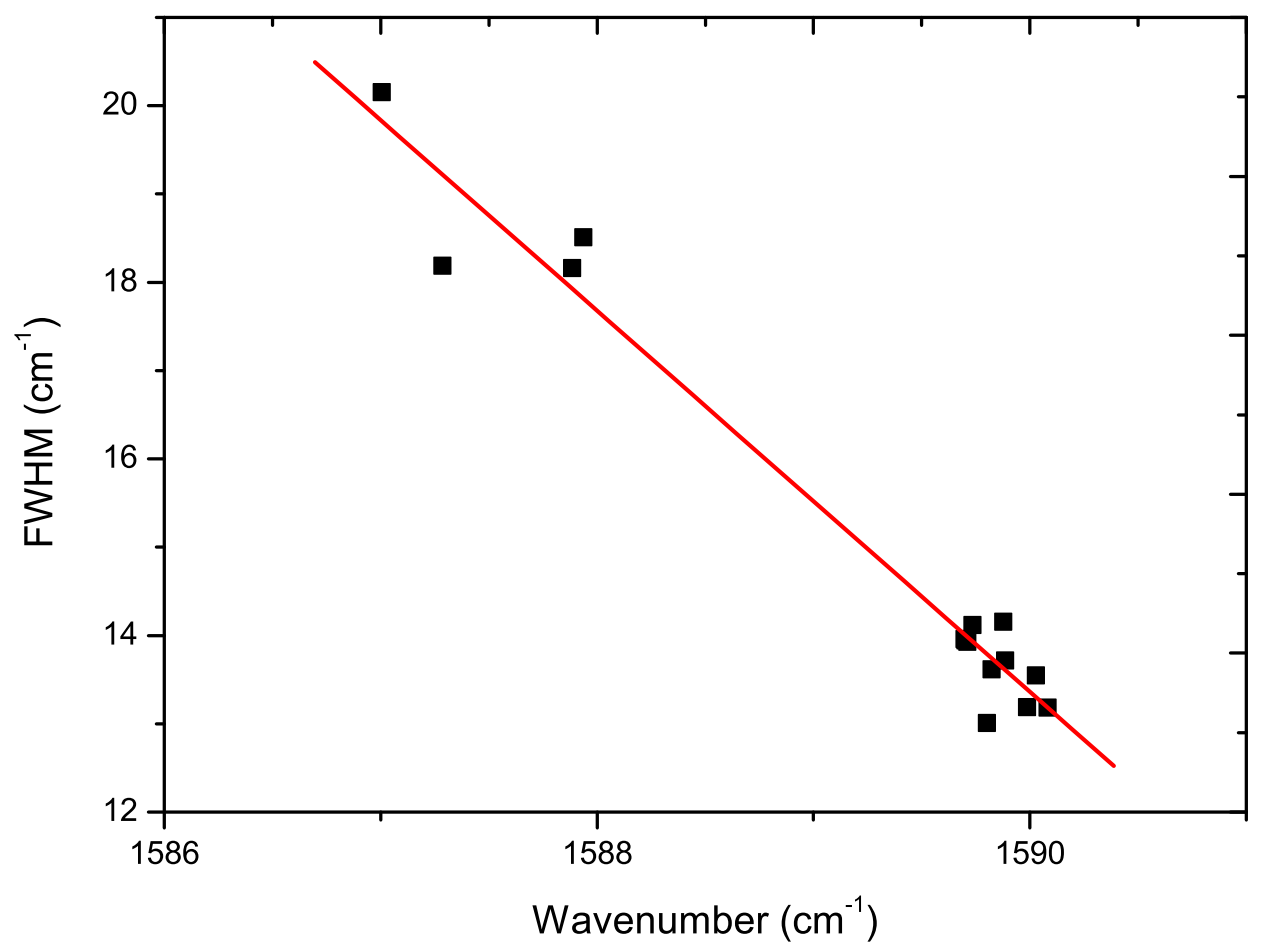

Figure 5.16: Linewidth or FWHM vs. frequency for the $\mathrm{G}^{+}$peak. The linear trend indicates that as frequency decreases the linewidth increases.

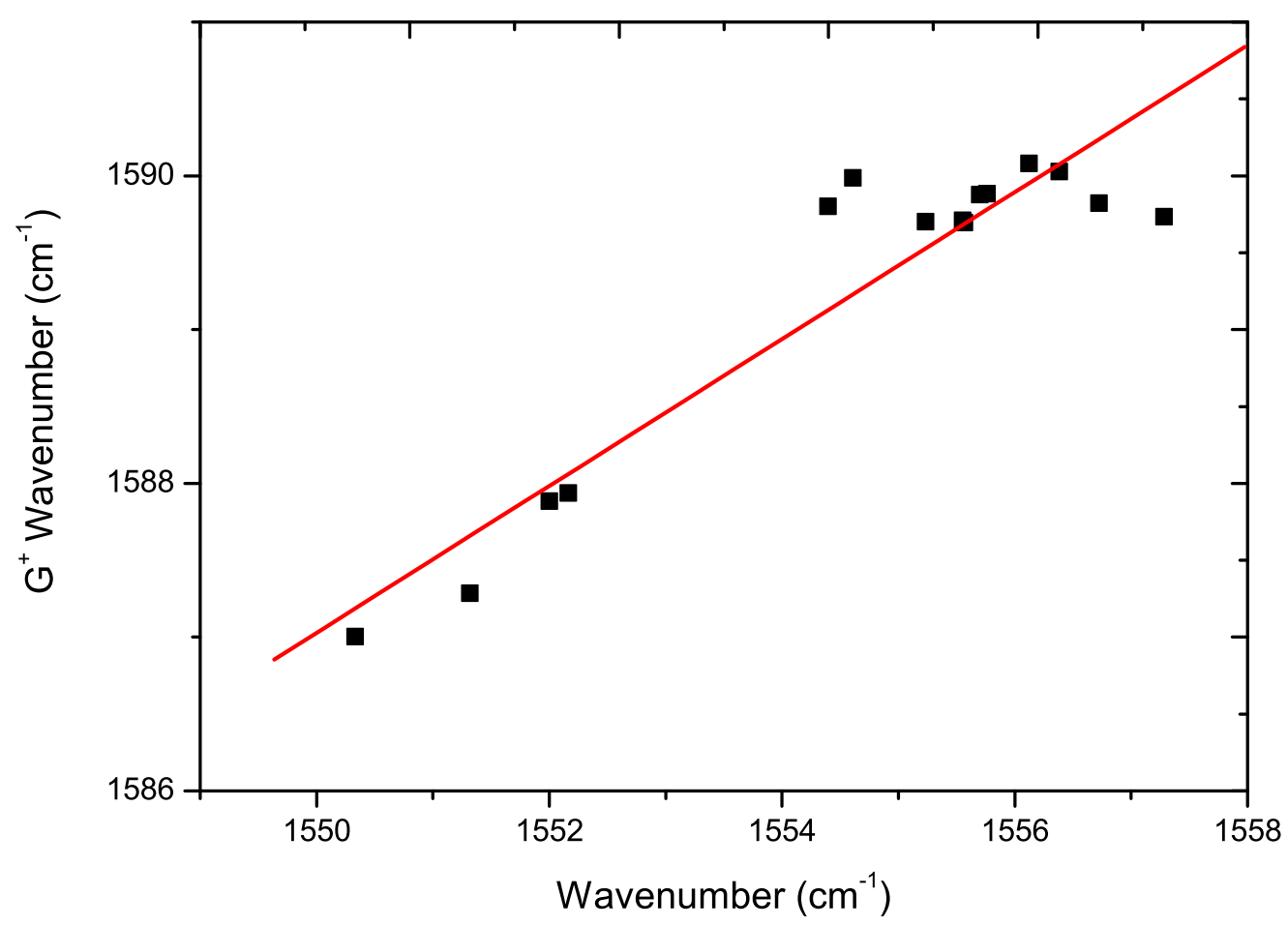

Figure 5.17: Frequency of the $\mathrm{G}^{+}$mode compared to the $\mathrm{G}^{-}$mode. 


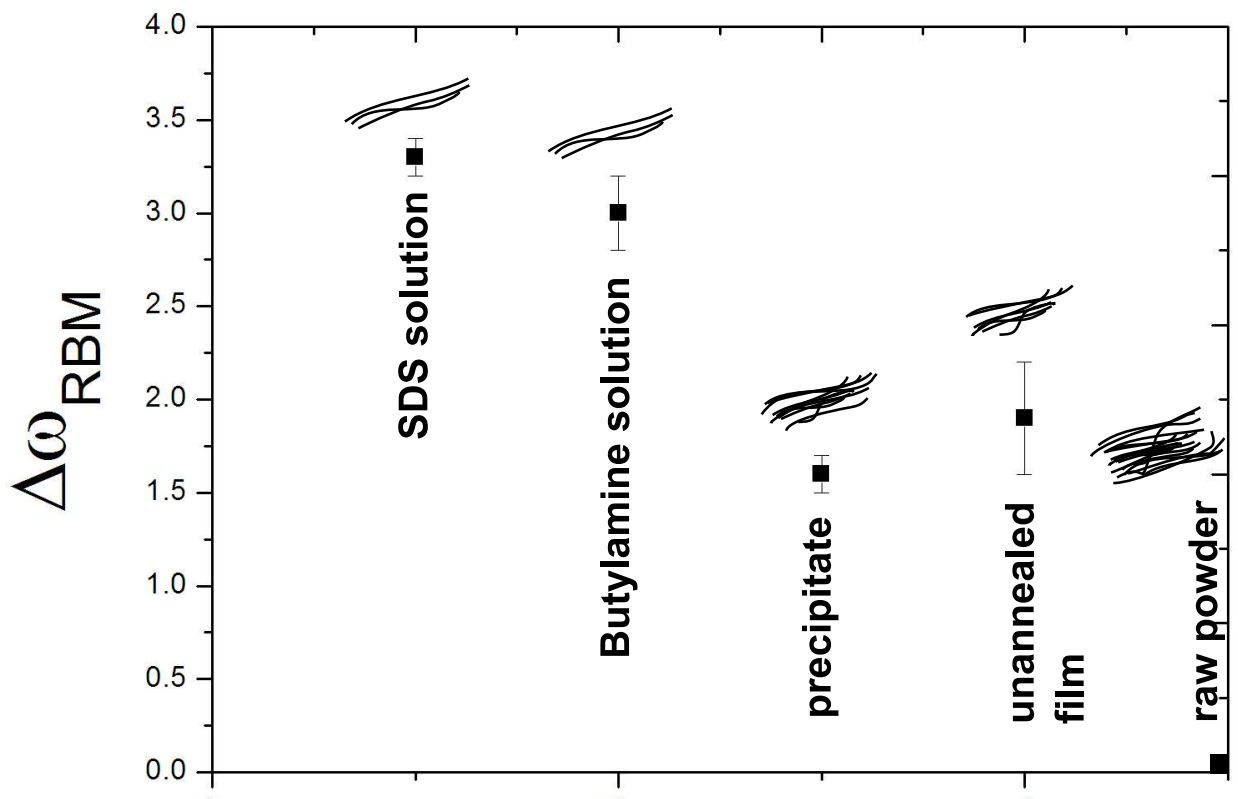

Figure 5.18: Summary of the Raman shifts in various samples compared to the raw HiPCO powder.

bundling-induced frequency downshift can be seen when the frequency is compared to the peak linewidth. The latter has been shown to broaden considerably for bundled nanotubes [119]. Figure 5.16 shows a plot of the $\mathrm{G}^{+}$linewidth against the peak frequency. Again, a linear trend is observed indicating that the peak frequency is dependent upon the extent of bundling. Figure 5.17 shows that the downshift in frequency occurs for both $\mathrm{G}^{+}$and $\mathrm{G}^{-}$modes.

In conclusion, we have established that unbundling induces an upshift in both the RBM and G-mode frequencies. Figure 5.18 shows a visual summary of the RBM shifts for all the samples studied in this section. With further analysis utilising multiple laser lines, it could be possible to use the absolute value of the shift as an alternative route to quantify the extent of bundling. 


\subsection{Thin films of SWNTs}

In Section 5.1.1, the integrated intensity was used to determine the relative population of a given electronic type. However, this information no longer offers any insight once the solvent and surfactant evaporate causing the SWNTs to re-assemble into inhomogeneous bundles. The films are sufficiently thin that variations in bundle size and composition across the sample are observed (see Figure 5.19). However, the absence or appearance of specific peaks can be used to monitor effects of external treatments in bundled samples. In this study, we examine the effect of annealing on the population of nanotubes. The TGA data presented earlier (Section 4.2.2) showed two peaks at $267{ }^{\circ} \mathrm{C}$ and $439{ }^{\circ} \mathrm{C}$ were qualitatively attributed to surfactant removal and nanotube combustion respectively. A more quantitative analysis can be undertaken by tracking the visible RBM peaks of films annealed to $100{ }^{\circ} \mathrm{C}, 300{ }^{\circ} \mathrm{C}$ and $500{ }^{\circ} \mathrm{C}$ compared to an unannealed film.

\subsubsection{Annealing effects}

RBM spectra were obtained from three separate spots for each of four samples: an unannealed film and films annealed to $100{ }^{\circ} \mathrm{C}, 300{ }^{\circ} \mathrm{C}$ and $500{ }^{\circ} \mathrm{C}$. The composite peaks were deconvoluted using QRAP and the disappearance of the RBM frequencies was monitored as a function of annealing. Peaks that were consistent across all four samples were assigned from the experimental assignments of Maultzsch et al. [49] and Wiltshire et al. [120].

Figures 5.20 and 5.21 show Raman spectra of the annealed films obtained with the $632.8 \mathrm{~nm}$ and $514.5 \mathrm{~nm}$ laser lines. Both the unannealed and $100{ }^{\circ} \mathrm{C}$ films show the same number of RBMs though the relative intensities are variable 


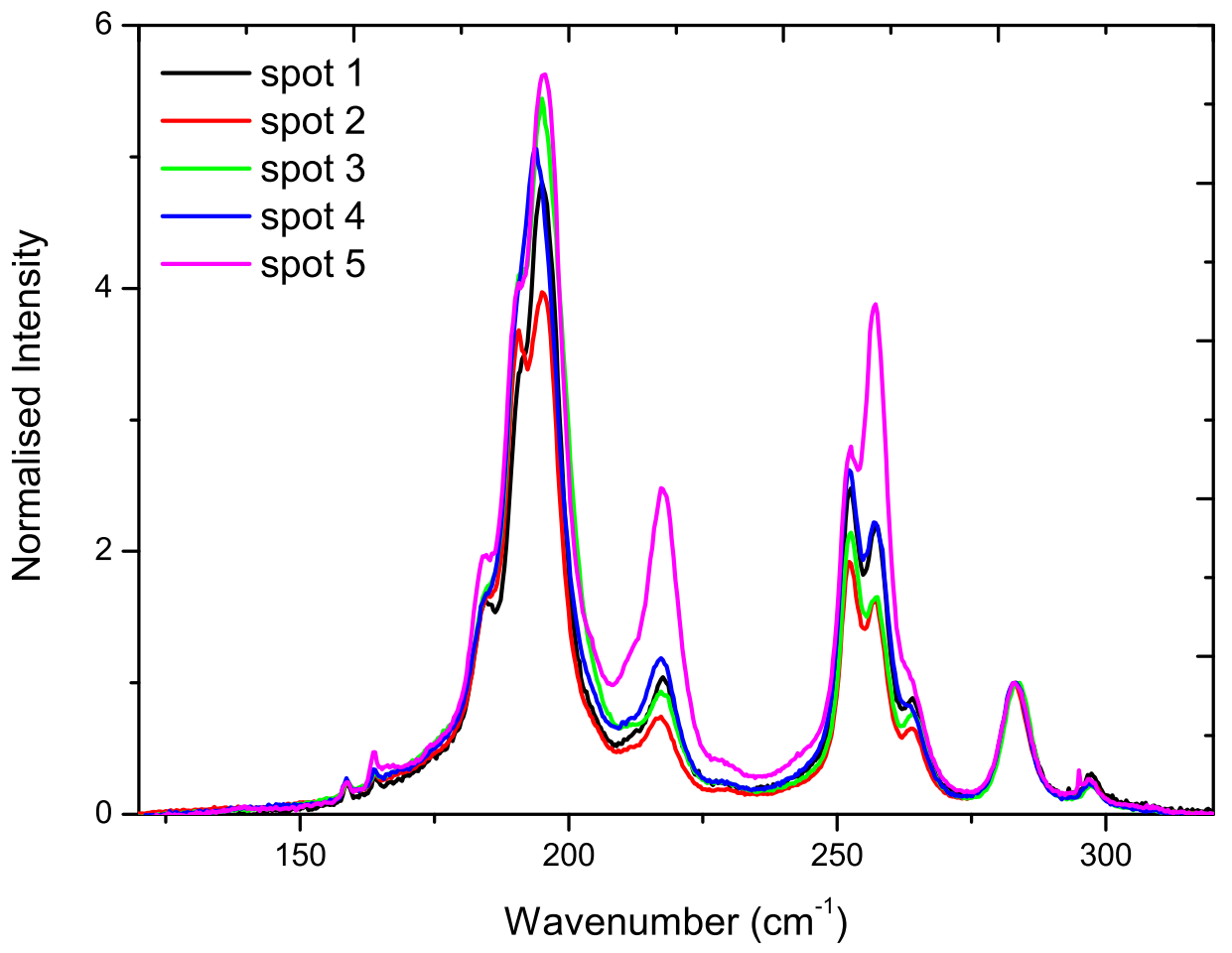

Figure 5.19: RBM spectra from five spots on an unannealed film taken under the $632.8 \mathrm{~nm}$ laser. The intensities are inconsistent due to the spatial inhomogeneity in bundle size.

due to spatial inhomogeneity. Higher frequency modes start to disappear even at an annealing temperature of $300{ }^{\circ} \mathrm{C}$ indicating that SWNTs corresponding to these modes have combusted upon annealing. Figure 5.21 shows a clear upshift in the RBM peaks of the film annealed at $500{ }^{\circ} \mathrm{C}$ whilst in Figure 5.20 the upshift is observed for films annealed to $300{ }^{\circ} \mathrm{C}$ and $500{ }^{\circ} \mathrm{C}$.

RBM spectra were collected from five spots on an unannealed and a film annealed to $300{ }^{\circ} \mathrm{C}$. The average frequency shift and uncertainty between the two films were calculated according to the methodology outline in Section 5.1.3. Our calculations yielded an average upshift of $3.3 \pm 0.3 \mathrm{~cm}^{-1}$ (see Figure 5.22). This can be attributed to a combination of two phenomena. The first is a reduction in bundle size due to nanotube combustion. Consequences of this phenomenon were presented in Section 5.2.1. Another possibility is a stiffening of the C-C bond dis- 


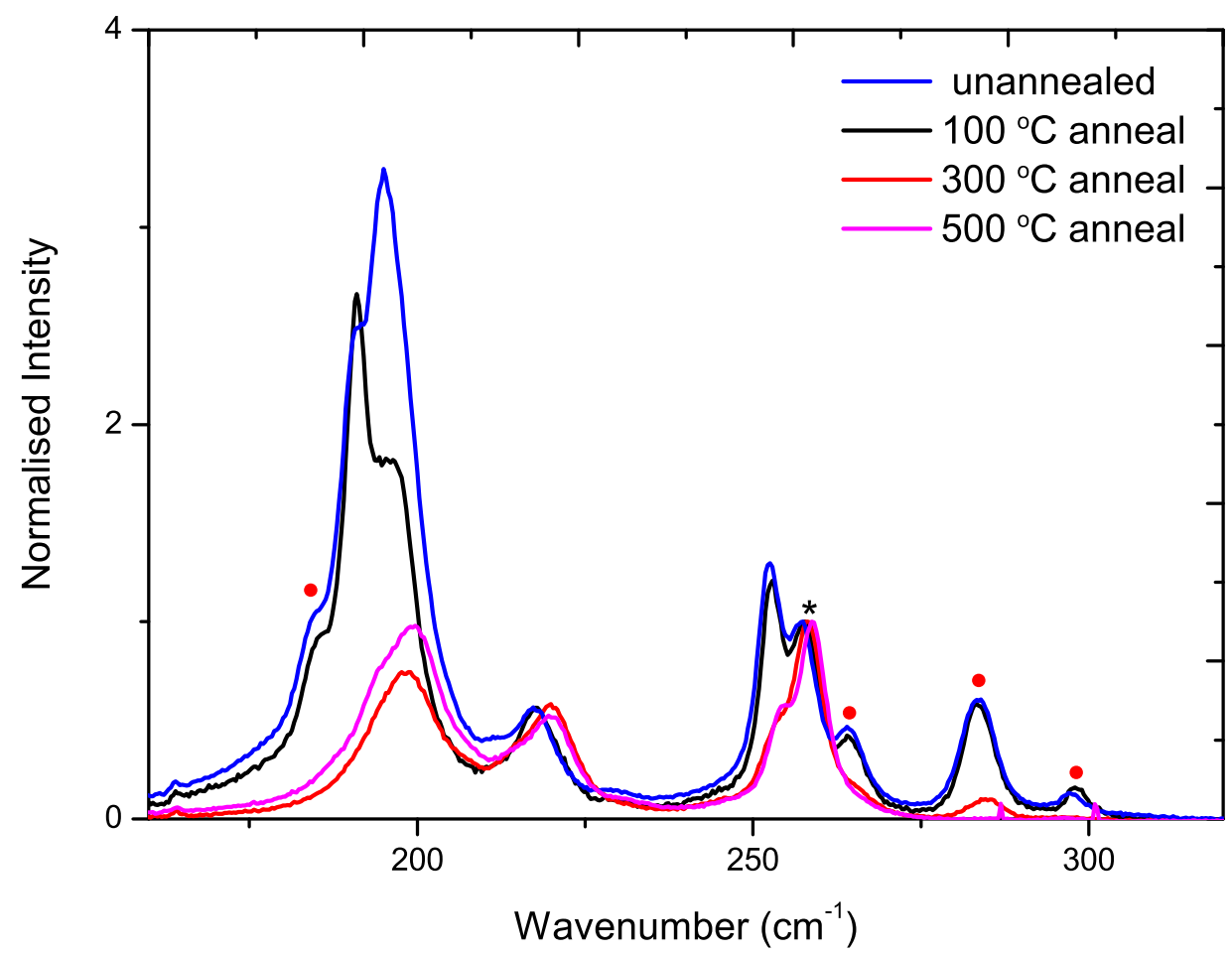

Figure 5.20: Raman spectra of annealed films under the $632.8 \mathrm{~nm}$ laser. The red dot indicates the peaks that disappear upon annealing.

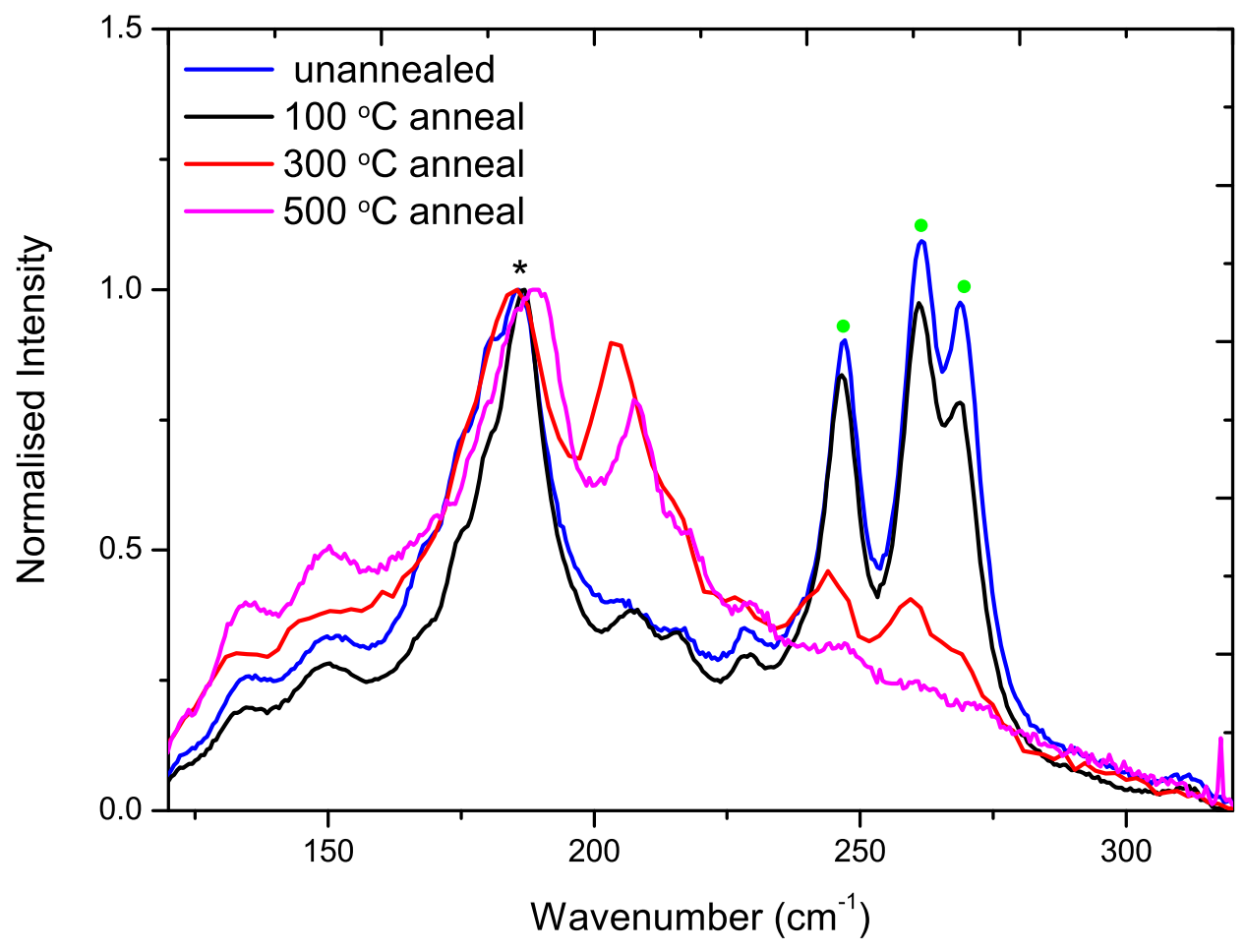

Figure 5.21: Raman spectra of the annealed films under the $514.5 \mathrm{~nm}$ laser. The green dot indicates the peaks that disappear upon annealing. 


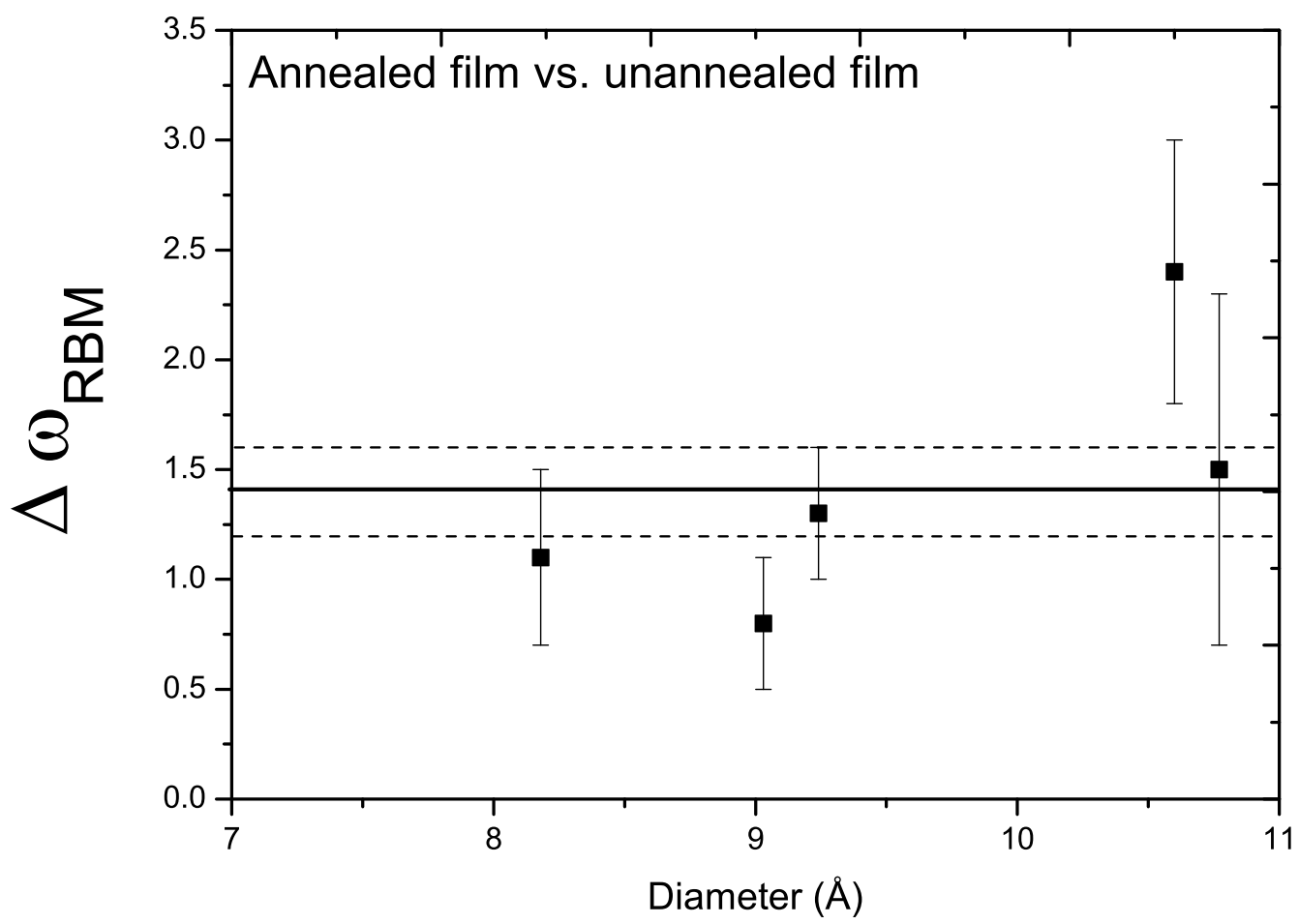

Figure 5.22: RBM shifts between unannealed and annealed films. The solid black line indicates the average shift while the dotted line indicates the uncertainty (standard error).

tance due to oxygen doping $[108,117]$ since the SWNT films were annealed in air.

Figure 5.23 shows the dependence of the combusted SWNTs as a function of diameter and chiral angle. Data from both lasers indicate that tubes with high chiral angle and small diameter are the first to combust. These tubes are expected to be more reactive due to their large pyramidalisation angle (Section 4.1). Our results match recent reports by Miyata et al. who showed that SWNTs of high chiral angle and small diameter were preferentially oxidised at $450{ }^{\circ} \mathrm{C}$ in air [121]. A similar study of oxidative combustion performed by Wiltshire et al. only showed a dependence on nanotube diameter [122].

While the general trend is clear from Figure 5.23, the reason behind the early combustion of the three tubes circled in black is not apparent. The same report 


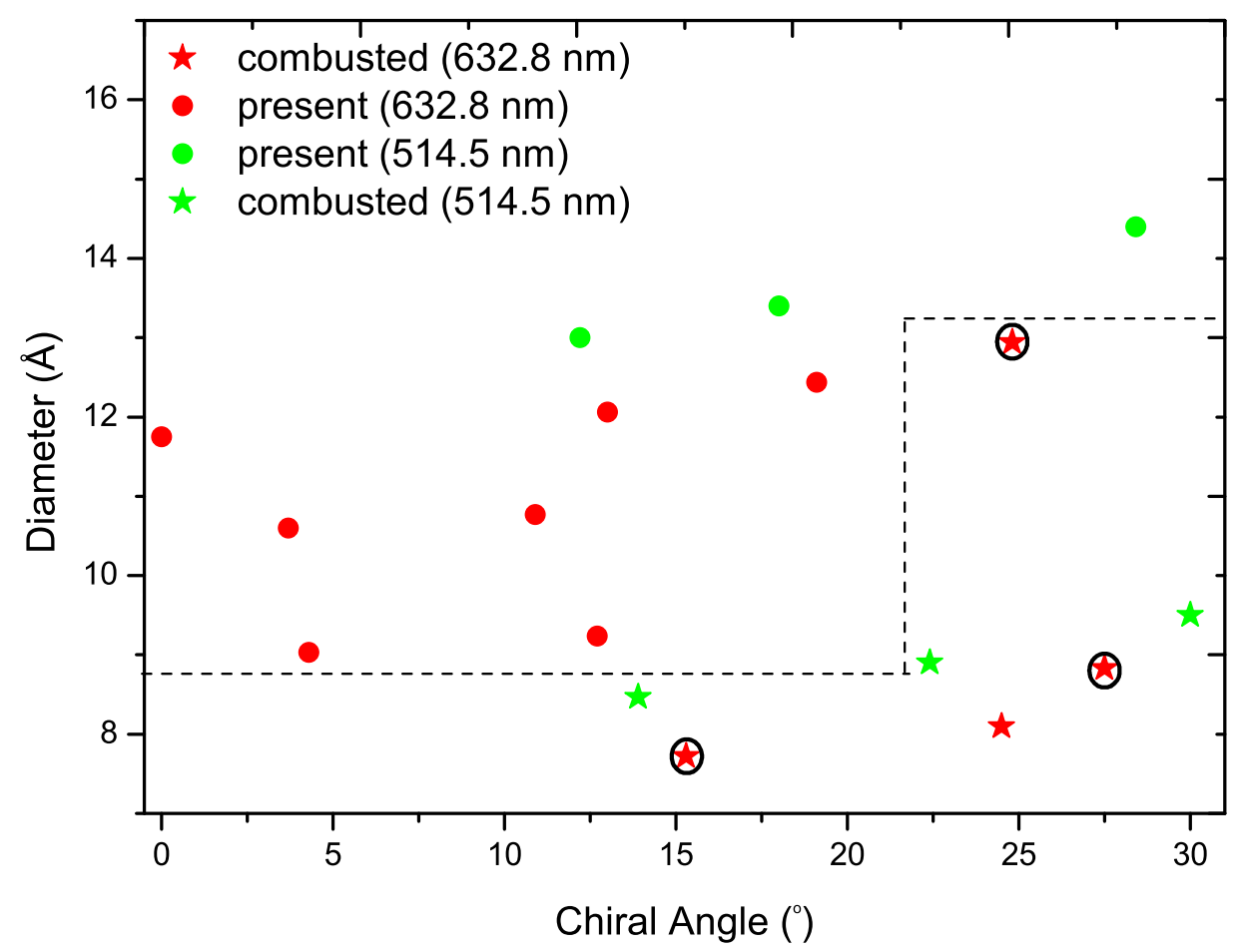

Figure 5.23: Diameter vs. chiral angle plot for films annealed at $300{ }^{\circ} \mathrm{C}$ and 500 ${ }^{\circ} \mathrm{C}$. The black circles represent SWNT peaks that disappeared after the $300{ }^{\circ} \mathrm{C}$ anneal.

by Miyata et al. contained results of SWNT films fabricated from a dispersion in $\mathrm{H}_{2} \mathrm{O}_{2}$ which combusted at a lower temperature [121]. Both $\mathrm{H}_{2} \mathrm{O}_{2}$ and butylamine are n-doping surfactants. Perhaps, a similar premature oxidation of SWNTs arises due to the presence of butylamine? Combustion in $\mathrm{H}_{2} \mathrm{O}_{2}$ was shown to be dependent on diameter while no systematic pattern was observed in the prematurely combusted tubes from our annealed networks. Using the RBM data only, the three black circles show no dependence on diameter, chiral angle or electronic type. However, the visible modes in the G-band in a sample annealed to $300{ }^{\circ} \mathrm{C}$ could arise from tubes of low chiral angle (see Section 5.1.3). This result hints at selective combustion of high chiral angle tubes during the $300{ }^{\circ} \mathrm{C}$ anneal. No other claims can be made beyond this due to a lack of conclusive evidence.

At this stage, the similarities between the work of Miyata et al. [121] and 
our system are qualitative but they match. Further study is required to fully explore the unresolved effect of butylamine on premature SWNT combustion. At present, it suffices to say that this method is effective in paring down the population of SWNTs in a systematic manner. With a little more work, this selective combustion could be applied as a technique to create enriched fractions of SWNTs with a narrow range of both chiral angle and diameter.

\subsection{Conclusion}

We were able to correlate small upshifts $\left(\sim 3 \mathrm{~cm}^{-1}\right)$ in $\mathrm{RBM}$ frequency between the powder and dispersed solution to unbundling. Centrifuged butylamine dispersions exhibited $\mathrm{a} \sim 3 \mathrm{~cm}^{-1}$ upshift in RBM frequency compared to the raw powder. RBM frequencies obtained from the well-dispersed SDS solutions of Maultzsch et al. [49] were also upshifted by the same amount from the powder. The G-band modes also showed a frequency shift of similar magnitude between heavily bundled and lightly bundled areas in our thin films. We speculate that these shifts occur due to a softening of the C-C bond upon aggregation.

Sonication in a butylamine/THF solution was found to exfoliate large aggregates down to small bundles. The extent of exfoliation was found to be independent of surfactant concentration. We also found our alkylamine/THF system to be non-selective by electronic type. This null result contradicts previous findings that reported enrichment of metallic [18, 19] and semiconducting SWNTs. [98]. However, the reason for the observed non-selectivity is not yet understood.

Nanotube combustion was implicated as the cause of the observed weight loss at $439{ }^{\circ} \mathrm{C}$ in the thermograms shown in Section 4.2.2. Our results show that nanotubes of high chiral angle and small diameter are selectively oxidised. This 
trend agrees with the expected increase in reactivity as a function of chiral angle and inverse diameter as well as the combustion results of Miyata et al. [121]. However, we are unable to explain the premature combustion at $300{ }^{\circ} \mathrm{C}$. 


\section{Chapter 6}

\section{Conduction Mechanisms in}

\section{SWNT networks}

Models such as FIT and VRH are used to describe conduction in nanotube networks. FIT dominates in thick networks since electrons can pick highly conducting paths interrupted only by thin barriers. As the film thickness decreases, the percolating network is no longer comprised of purely metallic pathways and the transport mechanism changes from FIT to VRH. A similar change-over is observed with an increasing concentration of disorder i.e. defects or impurities. These changes lead to a different mechanism due to an increase in barrier energy that can no longer be reduced by fluctuating charge. Instead, electrons conduct by hopping between localised states.

The SEM images of annealed and unannealed networks fabricated in this study are depicted in Figure 6.1. The amount of residual surfactant in the unannealed samples can be qualitatively gauged from the global morphology. Layers of loosely woven SWNTs in as-prepared networks are spatially separated by excess surfactant in the unannealed sample shown in Figure 6.1 (c). The dark patch is surfactant residue pyrolysed by the electron beam of the SEM. When butylamine 


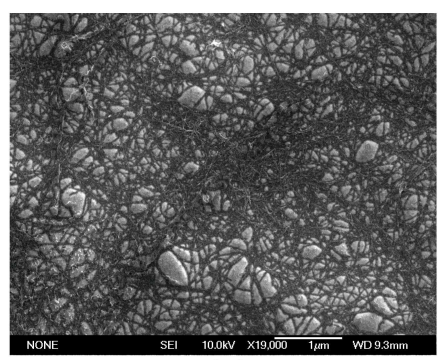

(a)

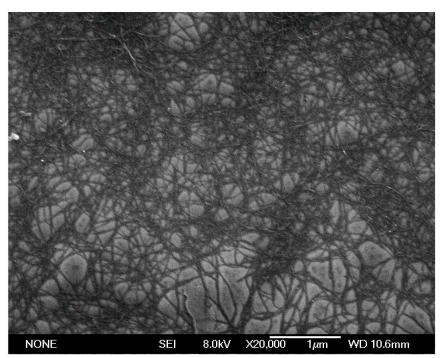

(b)

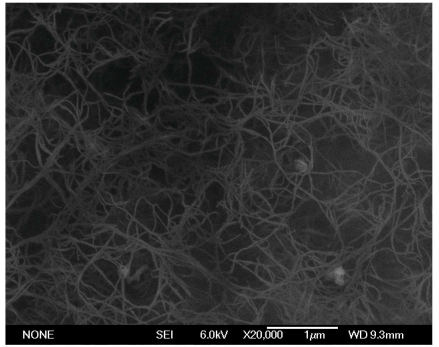

(c)

Figure 6.1: SEM images of SWNT networks at a magnification of 20,000 x. (a) Unannealed network prepared with a different batch of butylamine surfactant. (b) Annealed network. (c) Unannealed network.

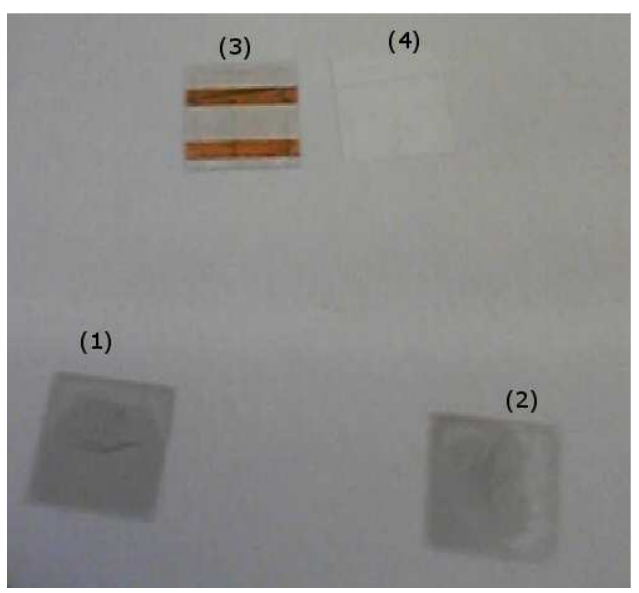

Figure 6.2: Photo of unannealed films both clean (3) and and cracked $(1,2)$ compared to a plain glass coverslip (4).

is desorbed from the nanotube sidewall after annealing to $300{ }^{\circ} \mathrm{C}$ (Figure 6.1 (b)), the SWNT layers contract and sit tightly against the substrate. Figure 6.1 (a) shows an unannealed sample fabricated from a different batch of butylamine. In this sample, we can see that there is little remnant surfactant since the network morphology is similar to the annealed sample in Figure 6.1 (b).

In a recent experimental study, de Andrade et al. [106] reported that residual surfactant not only deteriorates optical transparency but also sheet conductivity. The adsorbed surfactant creates a spatial barrier which hampers inter-tube electron transport [81]. Although we selected a volatile surfactant such as butylamine to reduce insulating spacers between SWNTs, some adsorbed surfactant still re- 
mains bound to the nanotube sidewall. Hence, we speculate that the amount of residual amine contributes to disorder in SWNT networks.

Another contribution to disorder could arise from inhomogeneity in the global morphology, e.g. homogeneous annealed (Figure $6.1(\mathrm{~b})$ ) and unannealed networks (Figure 6.1 (c) and Figure $6.2(3)$ ) compared with highly aggregated, cracked films (Figure 6.2 (1-2)). The latter have a mixed morphology comprised of fat ropes of SWNTs - visible to the naked eye - that lie within a thicker network. The thickness of these cracked networks is apparent from the high opacity.

This chapter will examine the underlying conduction mechanisms in annealed and unannealed networks. The conduction mechanism in unannealed networks (Section 6.1) is presented first before considering the effects of annealing on electron transport (Section 6.2).

\subsection{Unannealed samples}

In Section 2.3.3, we saw that the extent of localisation depends on the amount of disorder. In unannealed networks, we speculate that disorder is dependent on the amount of residual amine and mixed morphology. Hence, we compare two kinds of disordered networks (see Table 6.1 for names and description). The first type has a variable amount of residual surfactant (U1 and U3). The second comparison is between networks of homogeneous morphology (U1 and U3) and one with a mixed morphology (U2). Samples U1, U2 and U3 were all fabricated from a 7:1 THF: SWNT butylamine dispersion though variations in the amount and batch of the surfactant gave rise to three different morphologies. Sample U1 is typical of films fabricated from recently-prepared butylamine dispersions; U2 was prepared from a dispersion where the concentration of butylamine was insufficient (due to evap- 


\begin{tabular}{|l|l|}
\hline Sample & Description \\
\hline \hline U1 & Clean unannealed sample prepared recently \\
\hline U2 & Roped unannealed sample \\
\hline U3 & Unannealed sample prepared earlier \\
\hline \hline
\end{tabular}

Table 6.1: Abbreviations and descriptions of unannealed samples.

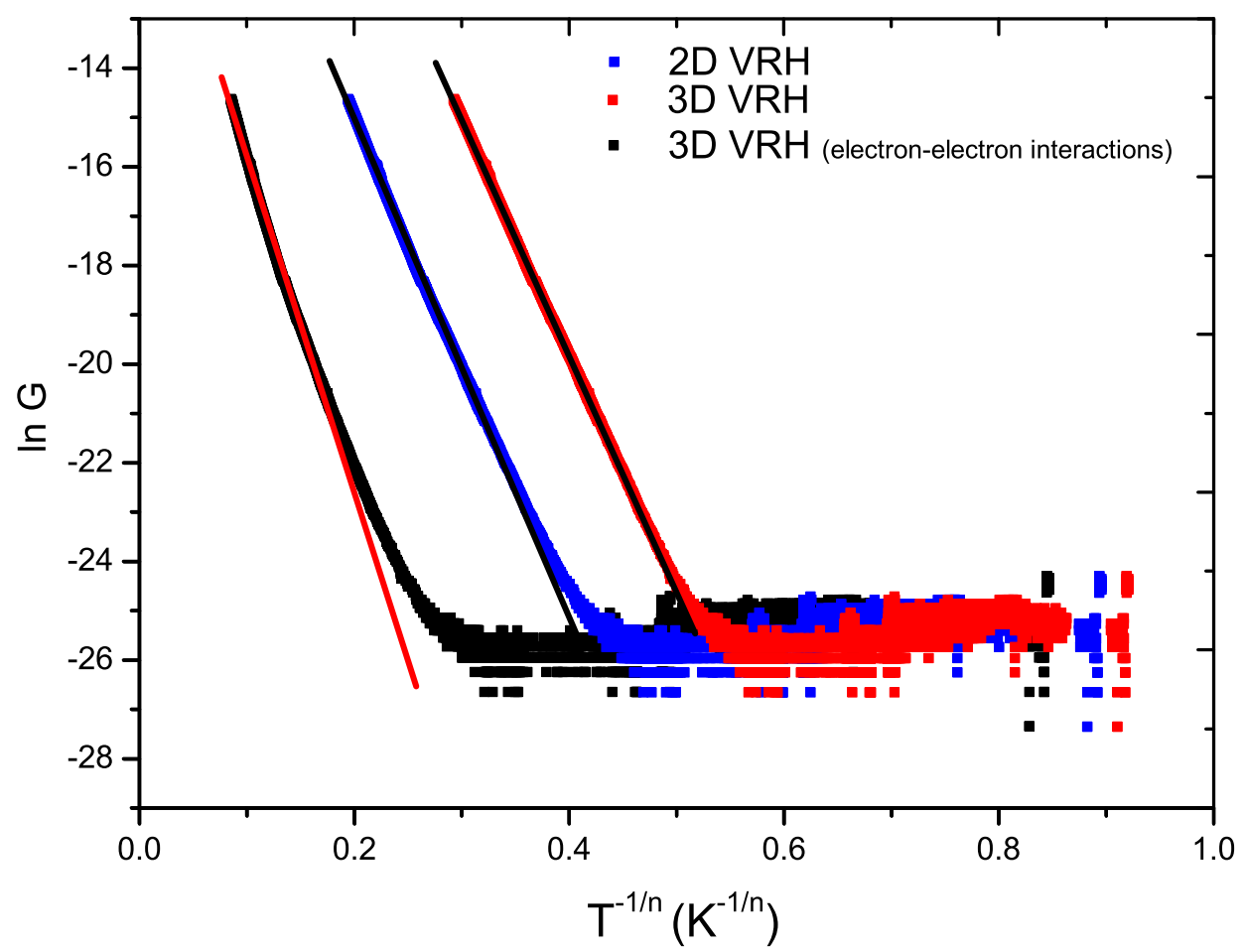

Figure 6.3: A comparison of different VRH mechanisms for U1. The natural log of the conductance is plotted against $\mathrm{T}^{-1 / n}$ where $\mathrm{n}$ is 2,3 and 4 for $3 \mathrm{D} \mathrm{VRH}$ with electron-electron interactions, 2D VRH and 3D VRH respectively.

orative losses from extended use) to prevent large scale agglomeration during the droplet drying process and U3 was prepared from a different batch of butylamine.

Temperature-dependent conductance data were obtained using the closedcycle cryostat for sample U3 and the glass cryostat for samples U1 and U2. The details of data acquisition are given in Sections 3.4.2 and 3.4.1. Due to limited time frame of liquid helium availability, some runs has be stopped before the temperature reached $300 \mathrm{~K}$. For example, data for U1 and U2 were only collected to $136 \mathrm{~K}$. 


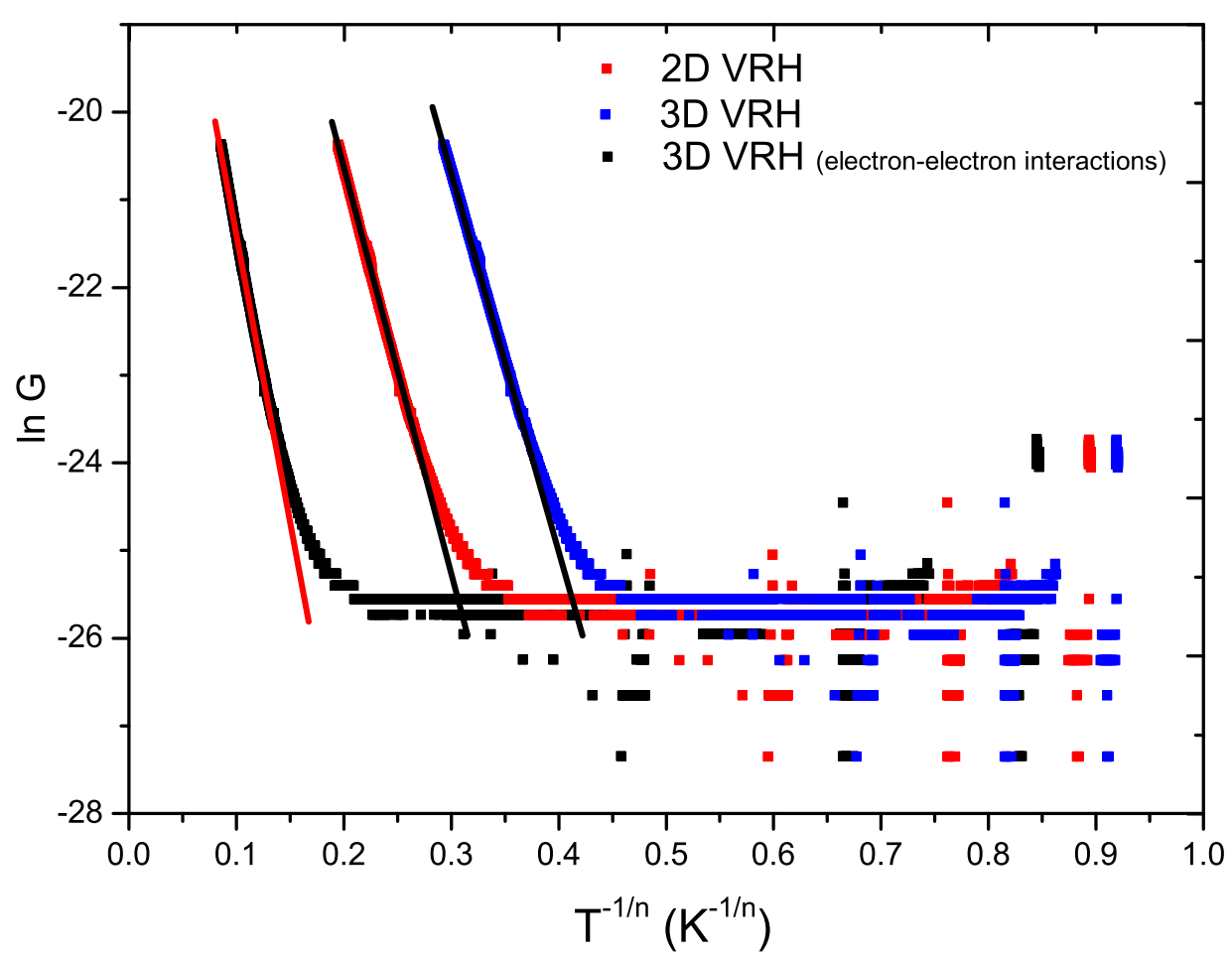

Figure 6.4: A comparison of different VRH mechanisms for U2. The natural log of the conductance is plotted against $\mathrm{T}^{-1 / n}$ where $\mathrm{n}$ is 2,3 and 4 for $3 \mathrm{D}$ VRH with electron-electron interactions, 2D VRH and 3D VRH respectively.

Conductance data were fit with three VRH models by linearising Equation 2.16. Figures 6.4, 6.3 and 6.5 show the log of conductance (ln $\mathrm{G}$ ) plotted against $1 / \mathrm{T}^{1 / 4}$, $1 / \mathrm{T}^{1 / 3}$ and $1 / \mathrm{T}^{1 / 2}$ for $3 \mathrm{D}$ VRH, $2 \mathrm{D}$ VRH and $3 \mathrm{D}$ VRH with electron-electron interactions respectively. For U1, 3D VRH is the only conduction mechanism that fits the $140 \mathrm{~K}-15 \mathrm{~K}$ temperature range. Below this range, the sample current reaches the detection limit of the multimeter and is indistinguishable from noise. The mixed morphology of U2 and the lack of valid data below $30 \mathrm{~K}$ for $\mathrm{U} 3$ are likely reasons for the ambiguous dimensionality (i.e. the dominant conduction mechanism is unclear) observed in Figures 6.4 and 6.5 respectively. We can resolve this ambiguity for $\mathrm{U} 3$ by association with the annealed sample, s4 (see Section 6.2). We suggest that 3D VRH is responsible for conduction since the annealed sample from the same batch of surfactant also follows this mechanism 


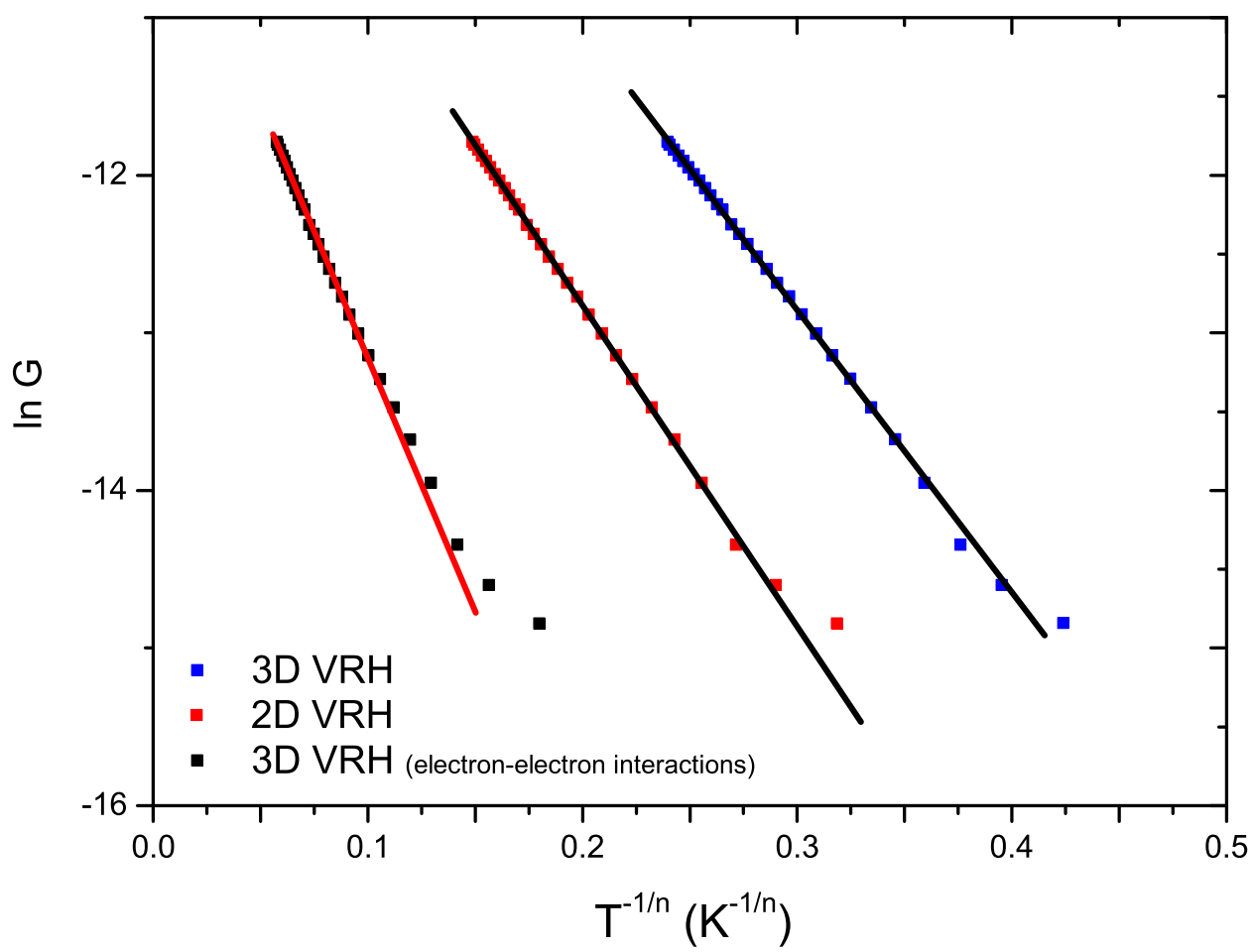

Figure 6.5: A comparison of different VRH mechanisms for U3. The natural log of the conductance is plotted against $\mathrm{T}^{-1 / n}$ where $\mathrm{n}$ is 2,3 and 4 for $3 \mathrm{D}$ VRH with electron-electron interactions, 2D VRH and 3D VRH respectively.

(Section 6.2).

The normalised conductance for samples U1, U2 and U3 is shown to $136 \mathrm{~K}$ in Figure 6.6. Since the unannealed samples follow VRH, the gradient of the conductance curves can be used to gauge the extent of localisation. In previous studies of SWNT networks, the localisation length was found to depend on the disorder concentration and thickness. In films with a small localisation length even a slight decrease in temperature changes the hopping distance since nearby localised states freeze out. This increase in hopping distance is marked by a corresponding decrease in conductivity. So, a steeper curvature of conductance as a function of temperature indicates a shorter localisation length. The steep gradient for U1 means that this sample has a smaller localisation length compared to 


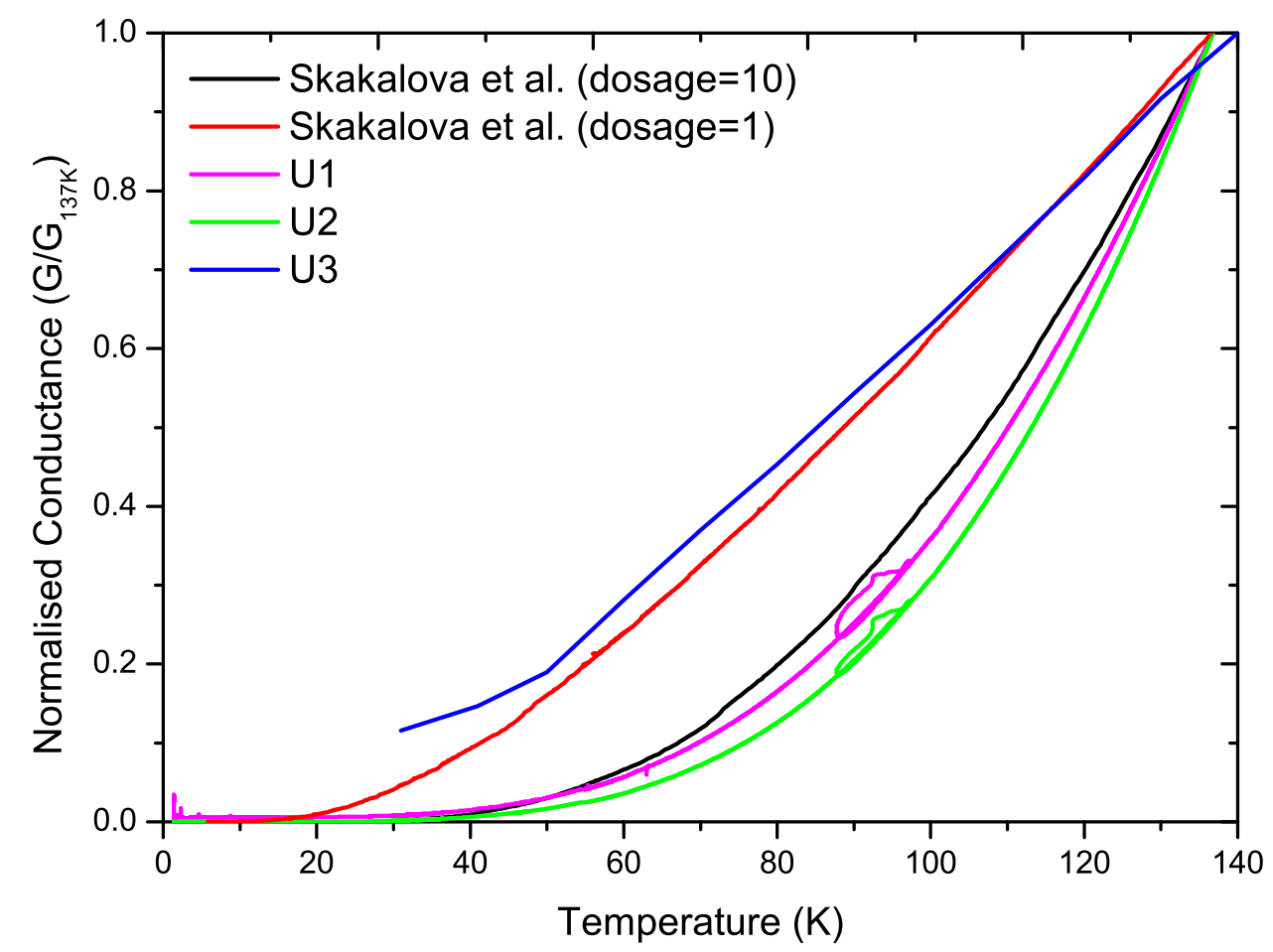

Figure 6.6: Normalised conductance vs. temperature for the unannealed samples compared to irradiated samples of Skakalova et al. [78].

$\mathrm{U} 3$.

Thick networks have a higher number of percolating metallic pathways with reduced inter-tube barriers compared to thinner networks. However, U2 shows the largest change in conductance with temperature in Figure 6.6. This indicates a greater degree of localisation in U2 compared to U3 though both were fabricated from similar solutions. Hence, the combined morphology of fat ropes in a thick mesh of nanotubes is implicated as another source of disorder.

We can infer the relative differences in the localisation length between different samples from the value of the $T_{0}$ parameter. The $T_{0}$ and $G_{0}$ parameters were found for both U1 and U3 by performing a Levenberg-Marquadt fitting algorithm on Origin using Equation 2.16. The values of $\mathrm{T}_{0}$ and $\mathrm{G}_{0}$ are given in Table 6.2.

$\mathrm{T}_{0}$ is proportional to the inverse cube of the localisation length, $\xi_{\text {loc }}$ (Equa- 


\begin{tabular}{|l|l|l|}
\hline Sample & $\mathbf{T}_{0}(\mathbf{K})$ & $\mathbf{G}_{0}$ \\
\hline \hline U1 & $5,368,209$ & $5.6 \times 10^{-1}$ \\
\hline U3 & 97,606 & $5.3 \times 10^{-4}$ \\
\hline \hline
\end{tabular}

Table 6.2: Parameters for U1 and U3 extracted from a 3D VRH fit.

tion 2.17). $\mathrm{T}_{0}$ has a much larger value in $\mathrm{U} 3$ indicating that the localisation length in this sample is much smaller than U1. Several studies have shown that the extent of localisation in SWNT networks can be tuned with ion irradiation $[123,124,78]$. The impact from the high energy ions induces increasing localisation with larger doses. For a qualitative comparison of the extent of localisation in our samples, we compare both U1 and U2 with irradiated networks of Skakalova et al. (Figure 6.6) [78]. Their SWNT networks were irradiated with varying doses of $\mathrm{Ar}^{+}$ions ${ }^{1}$. U1 shows a similar conductance curve to the network that has been irradiated with a high dosage of $\mathrm{Ar}^{+}$ions while $\mathrm{U} 3$ is a close match to the mildly irradiated network.

The only visible difference between U1 and U3 - from the SEM images of similar networks - is the amount of butylamine residue. But, these images are only a guide. The variability between samples in random, entangled networks is due to many factors including small changes in drop-drying conditions and the dynamics of bundling in dispersions [125]. If we assume that the amount of residual amine was the cause of the variation in $\mathrm{T}_{0}$ between $\mathrm{U} 1$ and $\mathrm{U} 3$, the optoelectronic properties would follow the prediction of de Andrade et al. [106]. Close to room temperature $(289 \mathrm{~K})$, U1 has a sheet conductance of $3.3 \mu \mathrm{S}$ at a 50 $\mathrm{V}$ bias. At the same temperature, $\mathrm{U} 3$ has a sheet conductance of $7.2 \mu \mathrm{S}$ at a $10 \mathrm{~V}$ bias. However, both samples are $\sim 70 \%$ transparent at $550 \mathrm{~nm}$. If the amount of residual amine were the cause of increased disorder, then the optical transparency for U1 would have been considerably lower. Thus, the higher conductivity for U3 is due to other causes. Possibilities include an increased fraction of metallic tubes

\footnotetext{
${ }^{1}$ Figures using data from [78] were obtained from the co-author, Prof. A.B. Kaiser
} 
or even a thicker network.

\subsection{Annealed Samples}

In Section 4.2.2, the optoelectronic properties of unannealed networks were found to improve drastically upon annealing to $300{ }^{\circ} \mathrm{C}$. The resistance of the unannealed network dropped by at least a factor of $10^{2}$ with an accompanying $>20 \%$ increase in optical transparency. This removal implies that barriers to electron conduction will be significantly decreased in annealed samples compared to unannealed networks. To verify this prediction, we studied three annealed SWNT networks fabricated from the dispersion as U1. The samples are arbitrarily assigned as s1, s2 and s3.

Conductance data were obtained using the glass cryostat (Section 3.4.2). Figure 6.7 shows the normalised conductance for the annealed samples. A rapid rise in temperature from $4.2 \mathrm{~K}$ to $50 \mathrm{~K}$ prevented low temperature measurements for samples s1 and s2. This limited the range of consistent data from $50 \mathrm{~K}$ to $280 \mathrm{~K}$. Due to limited availability and time constraints associated with the liquid helium, data for sample s3 were only collected from $1.3 \mathrm{~K}$ to $136 \mathrm{~K}$. Data between 30 $50 \mathrm{~K}$ for s3 were removed due to an experimental error where the multimeter was set to the wrong range. Thus, the normalisation was taken to $136 \mathrm{~K}$ for an equal comparison between the three samples.

Though only one complete range of data from $1.3 \mathrm{~K}$ to $280 \mathrm{~K}$ is possible from this series of experiments, the consistent behaviour of the annealed samples (Figure 6.7) ensures that this set will describe the universal behaviour of the annealed samples. Data from s3 provides the temperature range from $1.3 \mathrm{~K}$ to $136 \mathrm{~K}$ while 


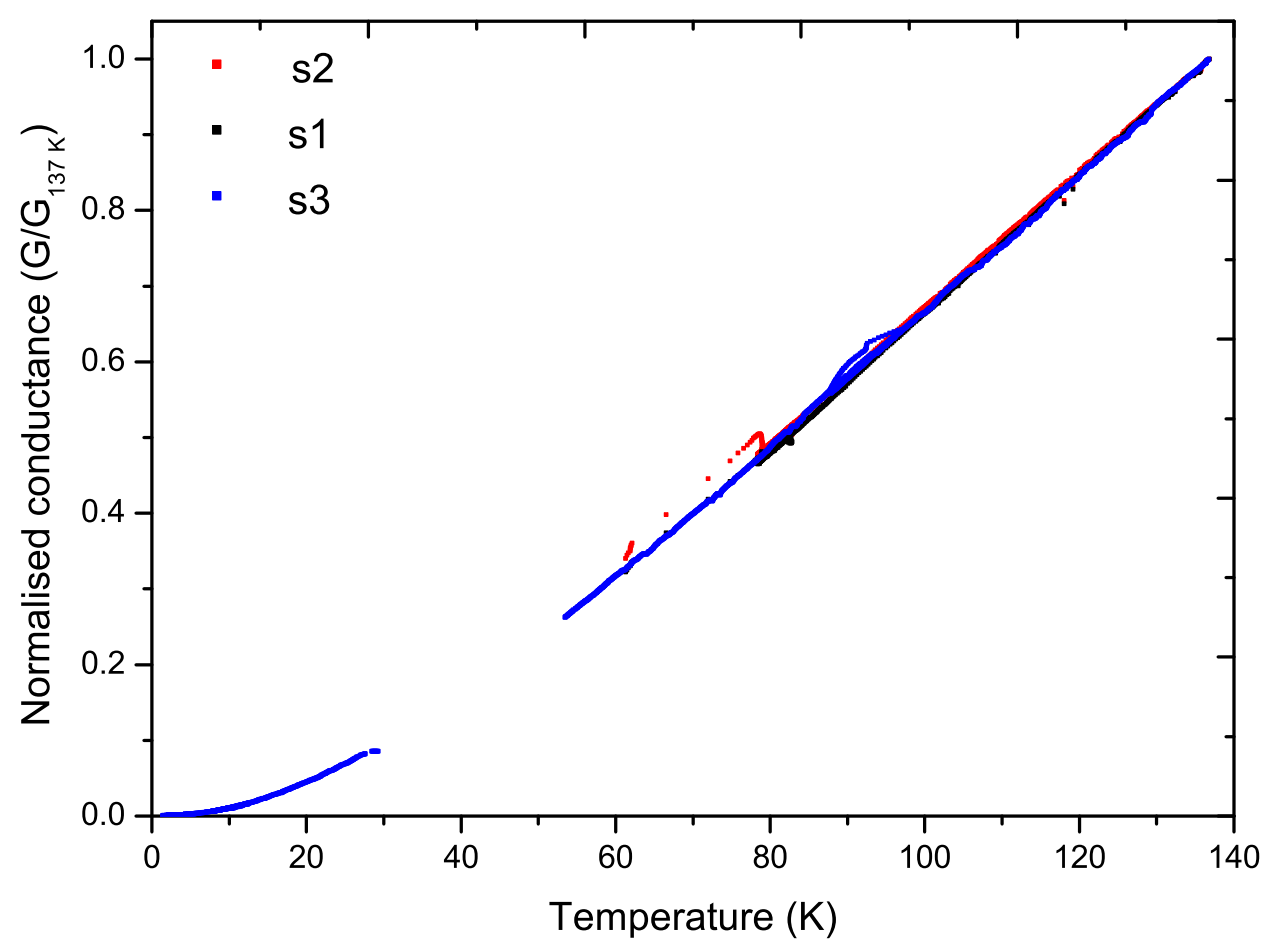

Figure 6.7: Graph of normalised conductance for all annealed samples.

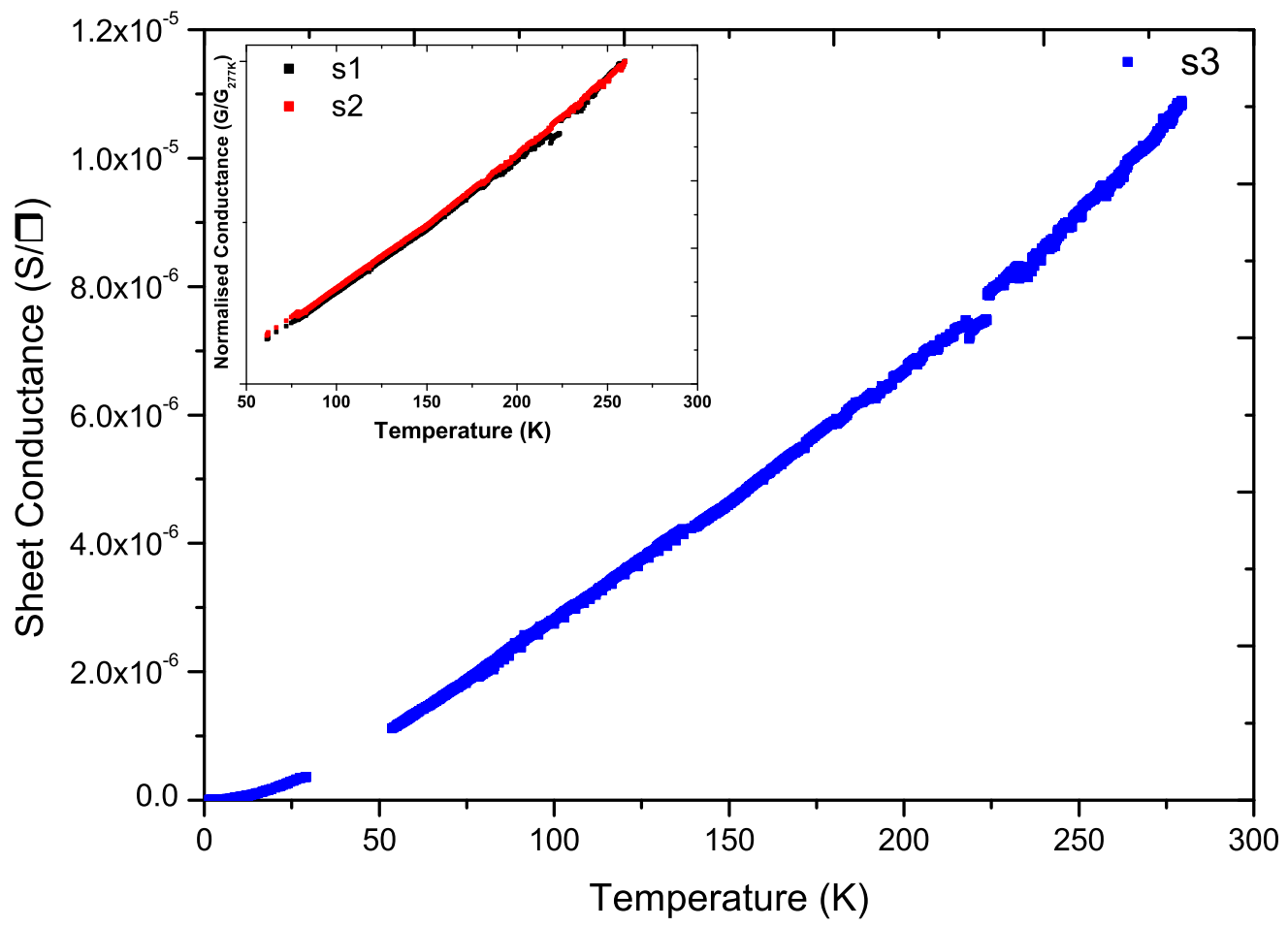

Figure 6.8: The full temperature range for sample s3. The inset shows that s1 and s2 continue to increase in a similar fashion to $280 \mathrm{~K}$. 


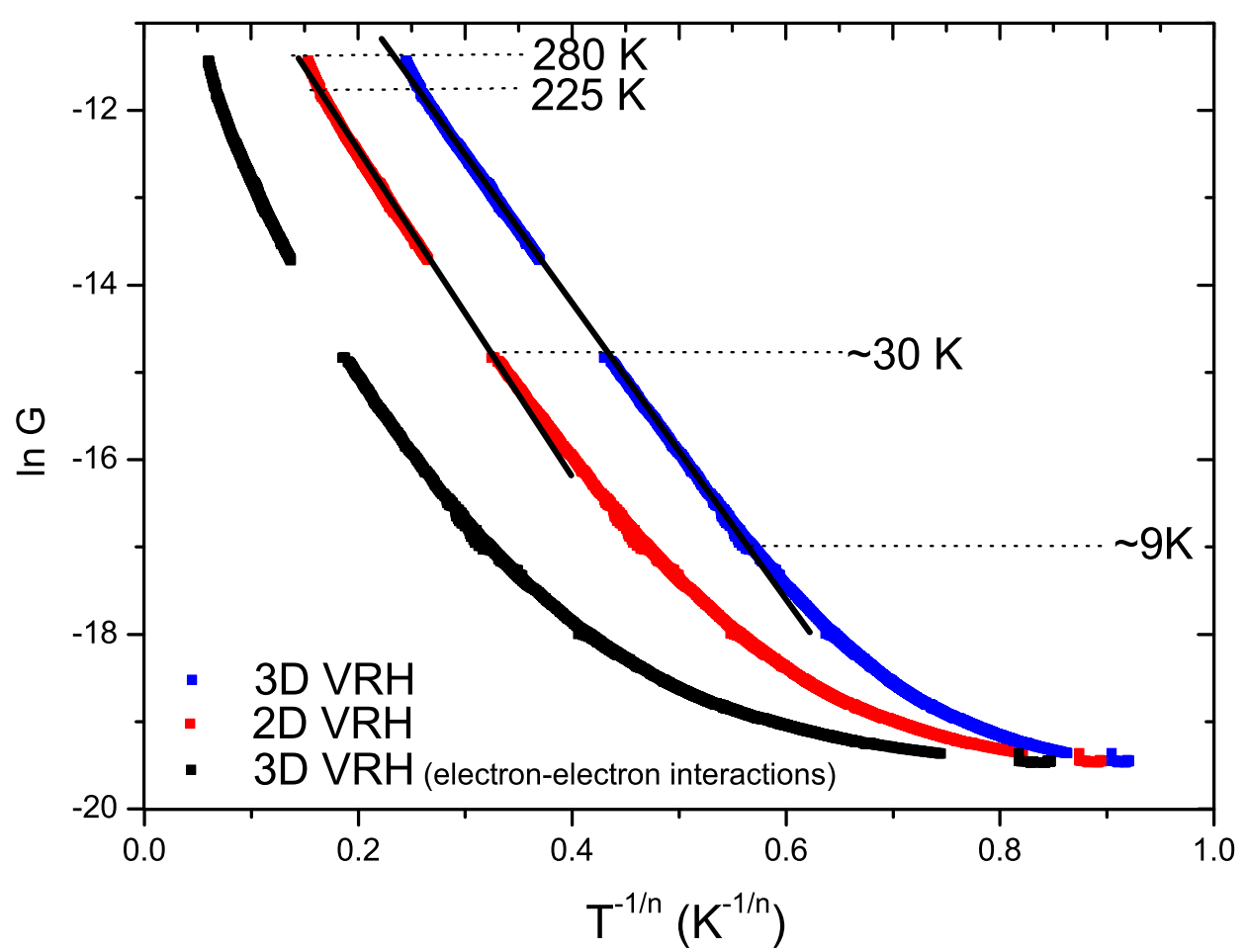

Figure 6.9: The natural log of conductance is plotted against $\mathrm{T}^{-1 / n}$ where $\mathrm{n}$ is 2 , 3 and 4 for 2D VRH with electron-electron interactions, 2D VRH and 3D VRH respectively.

data from s1 for between $137 \mathrm{~K}$ and $280 \mathrm{~K}$ is scaled down by a factor of 0.6 for an even continuation of s3 data. This scaled matching is consistent since both s1 and s2 lie on top each other when normalised to $280 \mathrm{~K}$ (inset to Figure 6.8). The complete range for s3 seen in Figure 6.8 is now sufficient for determining the mechanism(s) responsible for conduction in annealed samples.

S3 data was fitted with both FIT and VRH though the results for FIT are not presented here since the model failed to fit the data. Figure 6.9 shows the data plotted such that a linear trend would implicate that particular mechanism for the range over which the linearity continues. For 2D and 3D VRH, ln G (natural $\log$ of conductance) was plotted against $1 / \mathrm{T}^{1 / 3}$ and $1 / \mathrm{T}^{1 / 4}$ respectively. For 3D VRH with electron-electron interactions, ln $\mathrm{G}$ was plotted against 1/ $\mathrm{T}^{1 / 2}$. 


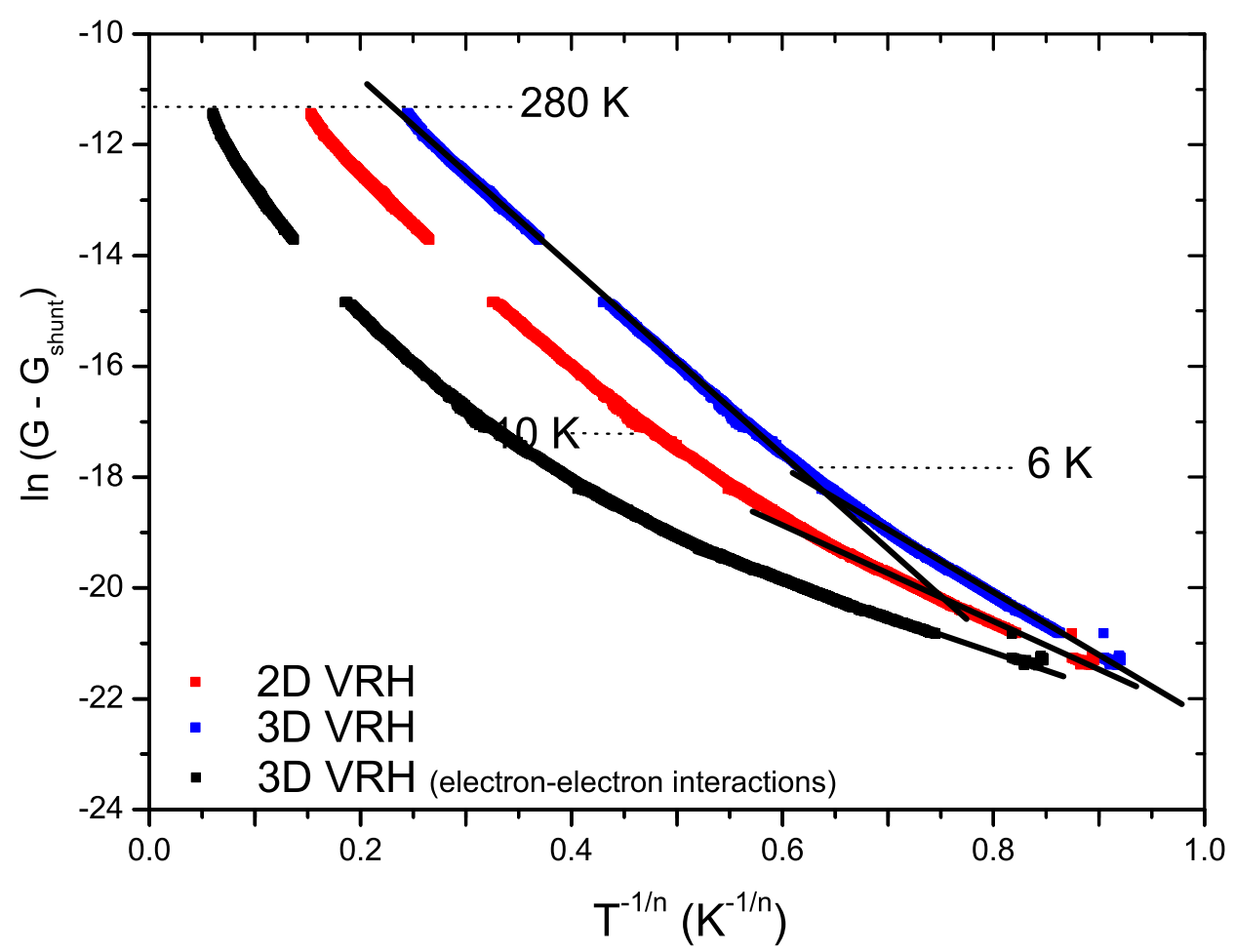

Figure 6.10: The effect of a subtracted shunt conductance on the three models depicted in Figure 6.9. The natural $\log$ of conductance is plotted against $\mathrm{T}^{-1 / n}$ where $\mathrm{n}$ is 2, 3 and 4 for $2 \mathrm{D}$ VRH with electron-electron interactions, 2D VRH and 3D VRH respectively.

From Figure 6.9, a linear trend for 3D VRH can be observed over a wide temperature range $(\sim 225-9 \mathrm{~K})$. The non-linear curvature of 2D VRH and 3D VRH with electron-electron interactions indicates that they are not responsible for conduction in the observed range (Figure 6.9). However, there is a divergence from the dominant 3D VRH mechanism which cannot be explained from this graph alone. The conductance flattens to some non-zero conductance below $9 \mathrm{~K}$ and exhibits a super-linear curve above $225 \mathrm{~K}$.

The conductance for s3 shown in Figure 6.9 saturates to a static value at very low temperatures. This residual or shunt conductance can be subtracted from the raw data to correct the curving divergence from the 3D VRH model. Accounting for such a shunt conductance straightens out the bottom leg of both the 3D VRH and 2D VRH models (Figure 6.10). The value of the shunt is optimised to below 
the extrapolated average of the static region. The optimisation was carried out by visual trial and error until the region below $10 \mathrm{~K}$ a followed an approximate linear trend. In this case, the average was $3.55 \mathrm{nS}$ and the optimised shunt conductance was $3 \mathrm{nS}$. This value is almost three orders of magnitude greater than the average leakage conductance of $4 \mathrm{pS}$ obtained from the unannealed sample (Figure 6.3).

The shunt can be viewed as a temperature-independent mechanism that is only visible at very low temperatures since the dominant VRH mechanism tends to zero as the temperature approaches $0 \mathrm{~K}$. Given the high interconnectivity of the base network, it stands within reason that small tunnelling paths between touching SWNTs exist within the heterogeneous ropes which contribute a small current even at very low temperatures. This intrinsic characteristic will be a static value that seems to 'turn on' at ultra low temperatures when VRH conduction decays to a negligible contribution.

As shown in Figure 6.10, both the 2D and 3D VRH models exhibit a linear trend at low temperature. The dimensionality of conduction within a SWNT network cannot be determined by physical means though spatial dimensionality can be traced by atomic force microscopy (AFM). Due to this open result, both $2 \mathrm{D}$ and $3 \mathrm{D}$ VRH have been fitted to the low temperature data (Figure 6.12).

Unlike many findings in current literature [77, 78, 79, 80], the annealed samples in this study do not follow one mechanism for the entire temperature range. For example, the thin networks of Skakalova et al. [78] that were mildly irradiated with $\mathrm{Ar}^{+}$ions show a conductance curve of comparable shape and curvature to s3. From a direct comparison of the two curves (Figure 6.11) we see that a linear trend is observed in the intermediate temperature range: between $50 \mathrm{~K}-225 \mathrm{~K}$ 


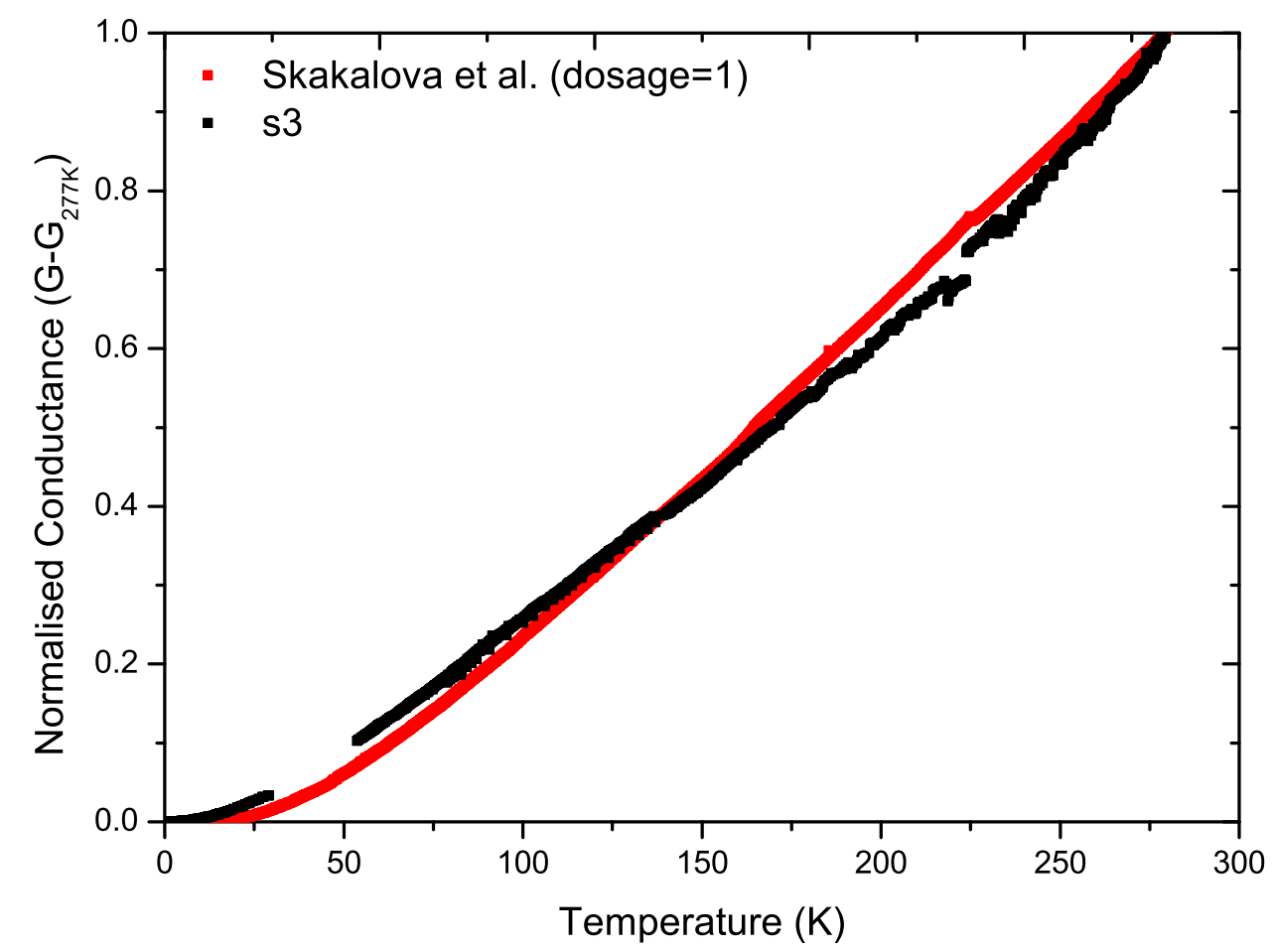

Figure 6.11: Normalised conductance vs. temperature for annealed sample s3 and a mildly irradiated sample [78].

for s3 and above $50 \mathrm{~K}$ for the irradiated network. However, s3 exhibits a steep increase in conductance above $225 \mathrm{~K}$ due to some high temperature mechanism while the irradiated sample continues along the linear trend to $300 \mathrm{~K}$.

Though 3D VRH is the dominant conduction mechanism for the range between 220 - $30 \mathrm{~K}$, we include other mechanisms such as the shunt conductance, the low temperature VRH which is either 2D or $3 \mathrm{D}$ and some high temperature mechanism to explain the entire temperature range. Since there are no sharp elbows in the conductance data, no abrupt changes in mechanisms are possible. This indicates that the mechanisms do not transform at a critical temperature. Instead, the different mechanisms contribute throughout the temperature range. Thus, a relation for the overall conductance can be written as the sum of four contributing parts: 


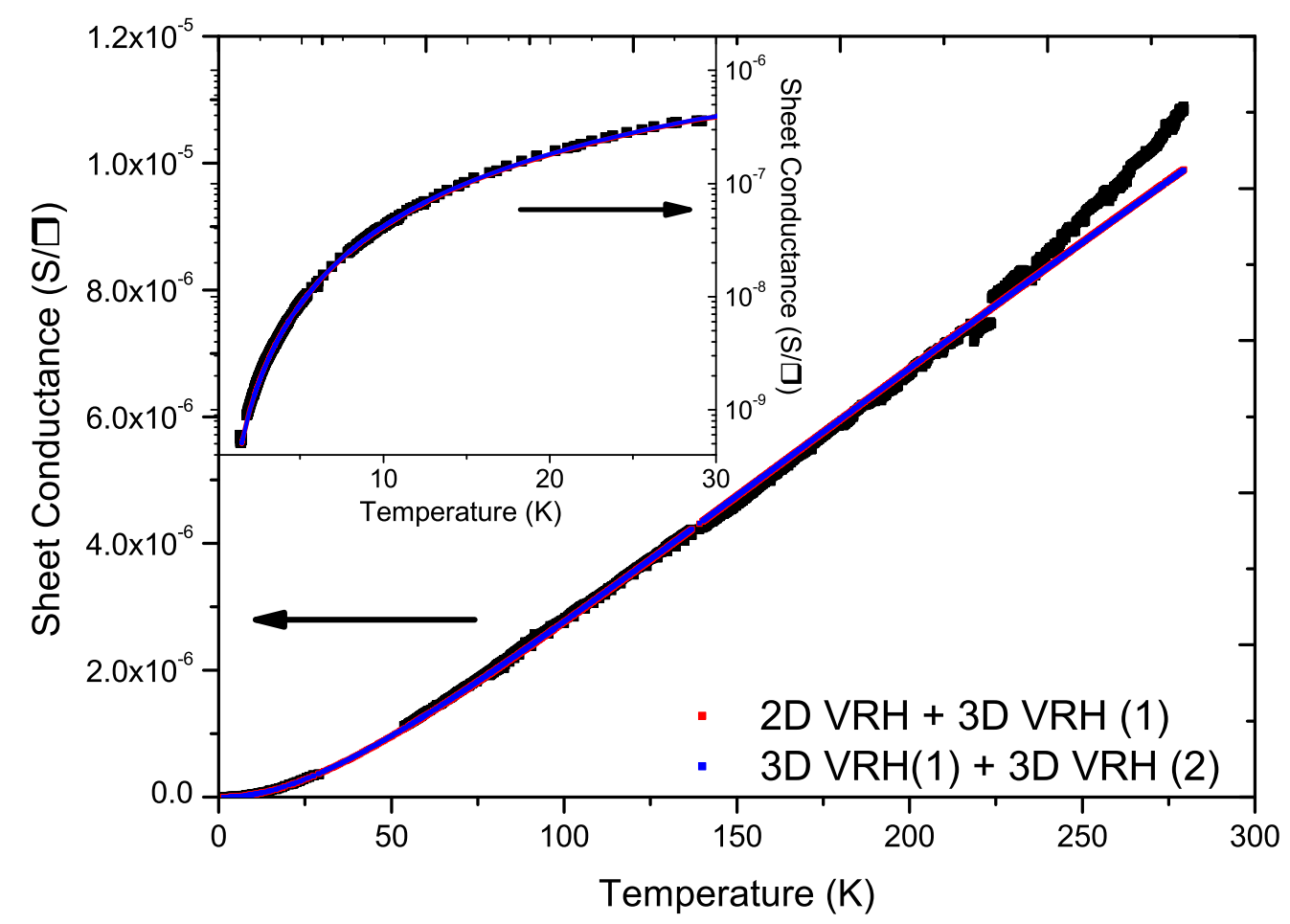

Figure 6.12: Graph showing the fitting results of a dual-regime VRH model. The blue curve depicts Model 1 where dominant conduction is 3D VRH while the low temperature mechanism is a combination of the dominant 3D VRH and a second type of 3D VRH. The red curve depicts Model 2 where 3D VRH dominates conduction at intermediate temperatures. At low temperatures, there is some contribution from 2D VRH conduction.

$$
G=\sum_{i}^{4} G_{i}=G_{\text {shunt }}+G_{\text {low temperature VRH }}+G_{3 D V R H}+G_{\text {high temperature }}
$$

To reduce the complexity of fitting four mechanisms at once, the shunt conductance was subtracted from the s3 dataset before the two VRH models were fitted. Model 1 incorporates a 2D VRH for the low temperature regime and 3D VRH to $225 \mathrm{~K}$ while Model 2 uses 3D VRH with different parameters to describe the low temperature region compared to the intermediate temperature regime. The equations for the two models are given in Table 6.3.

The parameters for the low temperature regime were obtained by fitting a 


\begin{tabular}{|l|l|}
\hline \multicolumn{2}{|l|}{ Model $\mathbf{1 :} \mathrm{G}_{1}=\mathrm{G}_{\text {shunt }} \mathrm{G}_{1} \exp \left(\left(T_{1} / T\right)^{1 / 3}\right)+\mathrm{G}_{2} \exp \left(\left(T_{2} / T\right)^{1 / 4}\right)$} \\
\hline $\mathbf{G}_{1}$ & $2.7 \times 10^{-7}$ \\
\hline $\mathbf{T}_{1}$ & $361 \mathrm{~K}$ \\
\hline $\mathbf{G}_{2}$ & $8.0 \times 10^{-4}$ \\
\hline $\mathbf{T}_{2}$ & $104776 \mathrm{~K}$ \\
\hline \hline & Model $\mathbf{2 :} \mathrm{G}_{2}=\mathrm{G}_{\text {shunt }}+\mathrm{G}_{3} \exp \left(\left(T_{3} / T\right)^{1 / 4}\right)+\mathrm{G}_{4} \exp \left(\left(T_{4} / T\right)^{1 / 4}\right)$ \\
\hline $\mathbf{G}_{3}$ & $1.5 \times 10^{-6}$ \\
\hline $\mathbf{T}_{3}$ & $6053 \mathrm{~K}$ \\
\hline $\mathbf{G}_{4}$ & $8.0 \times 10^{-4}$ \\
\hline $\mathbf{T}_{4}$ & $105309 \mathrm{~K}$ \\
\hline \hline
\end{tabular}

Table 6.3: Parameters for the two dual-regime VRH models depicted in Figure 6.12 .

linear trend to the elbow below $6 \mathrm{~K}$ for both $\ln \mathrm{G}$ vs $1 / \mathrm{T}^{1 / 3}$ and $1 / \mathrm{T}^{1 / 4}$ (see Table 6.3). The low temperature contribution was then subtracted from the conductance data and the natural log of the residual data was plotted against $1 / \mathrm{T}^{1 / 4}$ and fitted with a linear trend between $225 \mathrm{~K}$ and $10 \mathrm{~K}$. The parameters for the two regimes were optimised manually by minimising the percentage error to below $5 \%$ between the fit and the data using an iterative fitting procedure.

Once the parameters for the VRH region were determined, the additive conductance from the two contributing regimes for Models 1 and 2 were overlayed on the data (Figure 6.12). Both Figure 6.12 and the inset show a clean fit up to $\sim 225 \mathrm{~K}$ above which the VRH model proceeds almost linearly while the data exhibits a definite curvature. This divergence could imply the emergence of activated conduction where a rapid rise in conductance is observed above some activation temperature. So, the residual data from the VRH contribution was fitted between $200 \mathrm{~K}$ - $280 \mathrm{~K}$ using the Levenberg-Marquadt fitting algorithm of Origin to a model of the form:

$$
G=G_{0} \exp (-A / T)
$$




\begin{tabular}{|c|c|c|}
\hline \multicolumn{3}{|c|}{ Model 1: $\mathrm{G}_{\text {total }}=\mathrm{G}_{1}+\mathrm{G}_{5} \exp \left(-A_{5} / T\right)$} \\
\hline $\mathbf{G}_{5}$ & $\mathbf{A}_{5}$ & Energy $(\mathbf{m e V})$ \\
\hline $4.2 \times 10^{-3}$ & 2541.8 & 219.0 \\
\hline \hline Model 2: & $\mathrm{G}_{\text {total }}=\mathrm{G}_{2}+\mathrm{G}_{6} \exp \left(-A_{6} / T\right)$ \\
\hline $\mathbf{G}_{6}$ & $\mathbf{A}_{6}$ & Energy $(\mathbf{m e V})$ \\
\hline $8.8 \times 10^{-3}$ & 2532.3 & 218.2 \\
\hline \hline
\end{tabular}

Table 6.4: Activation parameters for fits shown in Figure 6.13.

where the constant A represents the activation energy and $G_{0}$ is a constant prefactor.

The combined fit for the two models including the high temperature activation (parameters given in Table 6.4) covers the entire temperature range for the annealed sample as seen in Figure 6.13. The two models provide an ample description for the four mechanisms governing conduction in annealed samples. Furthermore, the low percentage error (see Figure 6.14) between the fit and data allows the determination of physical parameters from the fit.

In the Section 2.3.3, localisation was stated to occur around defect sites. For the annealed samples, the intermediate temperature range $(30-225 \mathrm{~K})$ is sufficient to access the majority of the localised states within the network. As temperature decreases, the high energy localised states become inaccessible and only the low energy localised states remain active. Even though the VRH model takes into account this decrease in localised states as a function of temperature, the annealed samples follow a different VRH model at low temperatures.

Figure 6.15 shows that the higher temperature VRH mechanism veers away from the conductance data below $\sim 15 \mathrm{~K}$. Only an additive combination of the two VRH mechanisms fits the data. The two regimes have different $T_{0}$ parameters where $T_{0}$ for the high temperature regime is greater than $T_{0}$ for the low tem- 


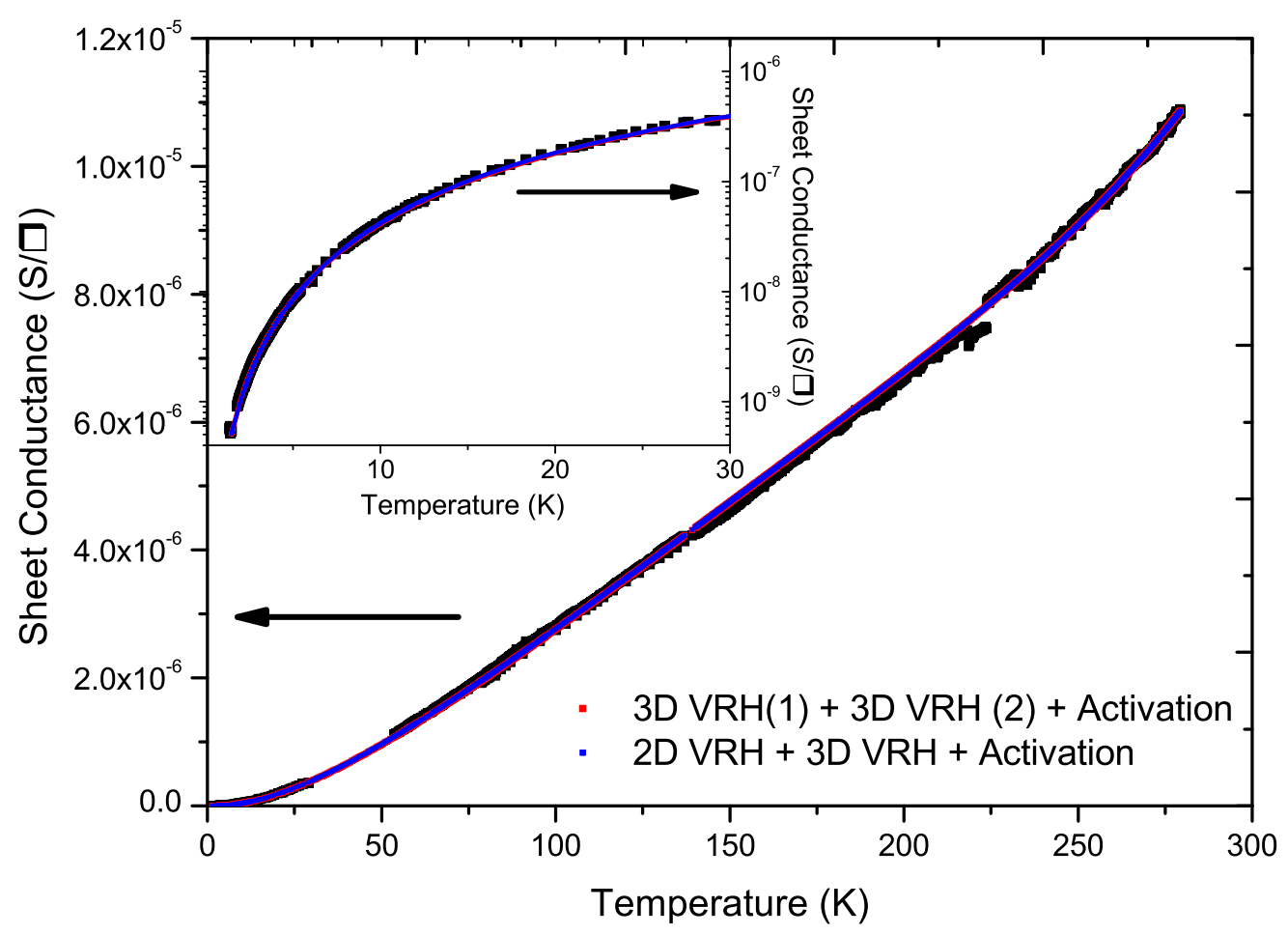

Figure 6.13: The combined fits including activation for the two models. The inset shows the region around the transition $(\sim 12 \mathrm{~K})$ where the additive contributions of VRH fall on top of the data points.

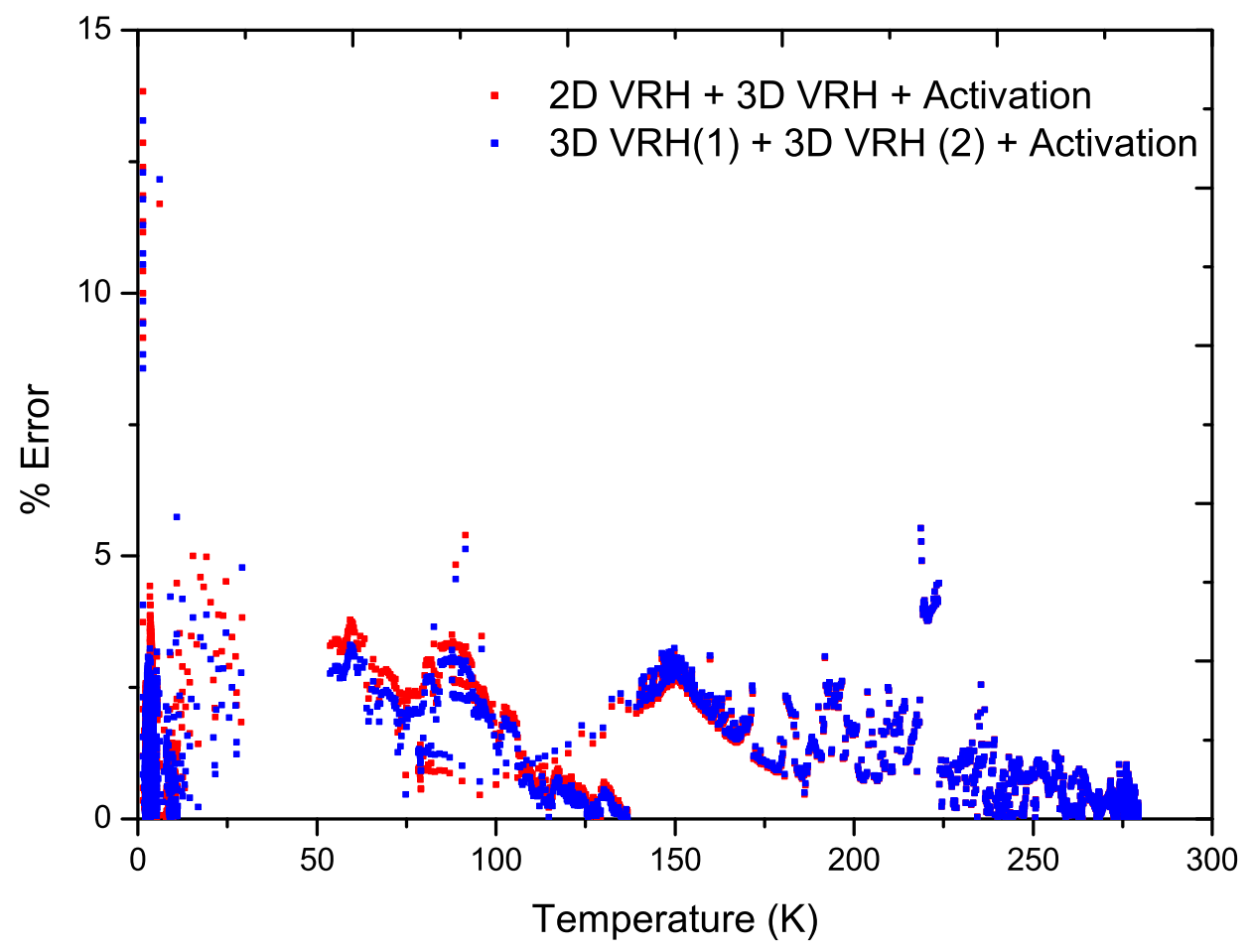

Figure 6.14: Percentage error between the fitted and raw data for the two models. With the exception of a few points at the lowest temperature, the iterative method optimises percentage errors to below $5 \%$. 


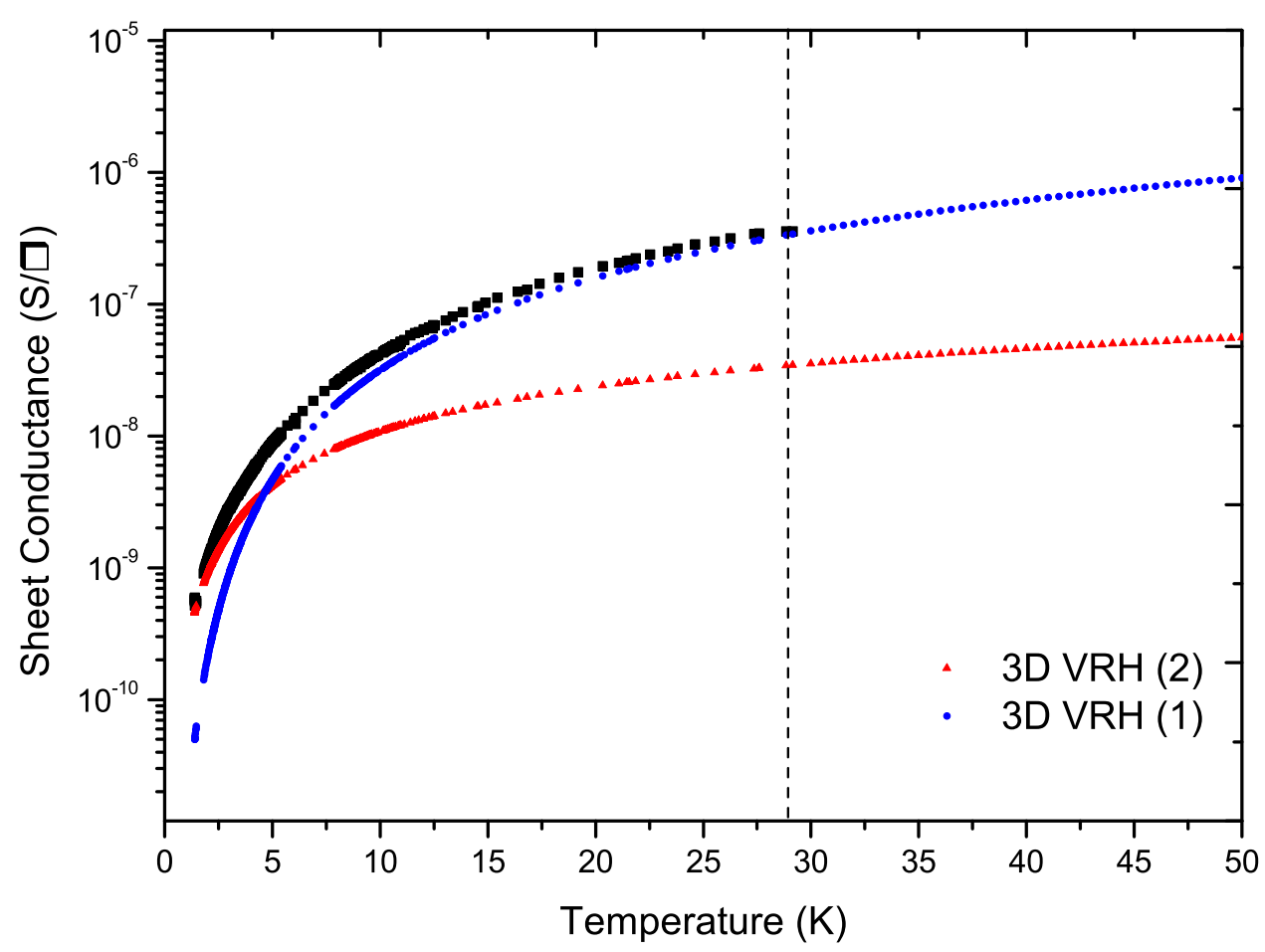

Figure 6.15: Only the additive combination of 3D VRH (2) and 3D VRH (1) can reproduce the curve of the data below $30 \mathrm{~K}$. Activation conduction provides no contribution to the conductance below $200 \mathrm{~K}$.

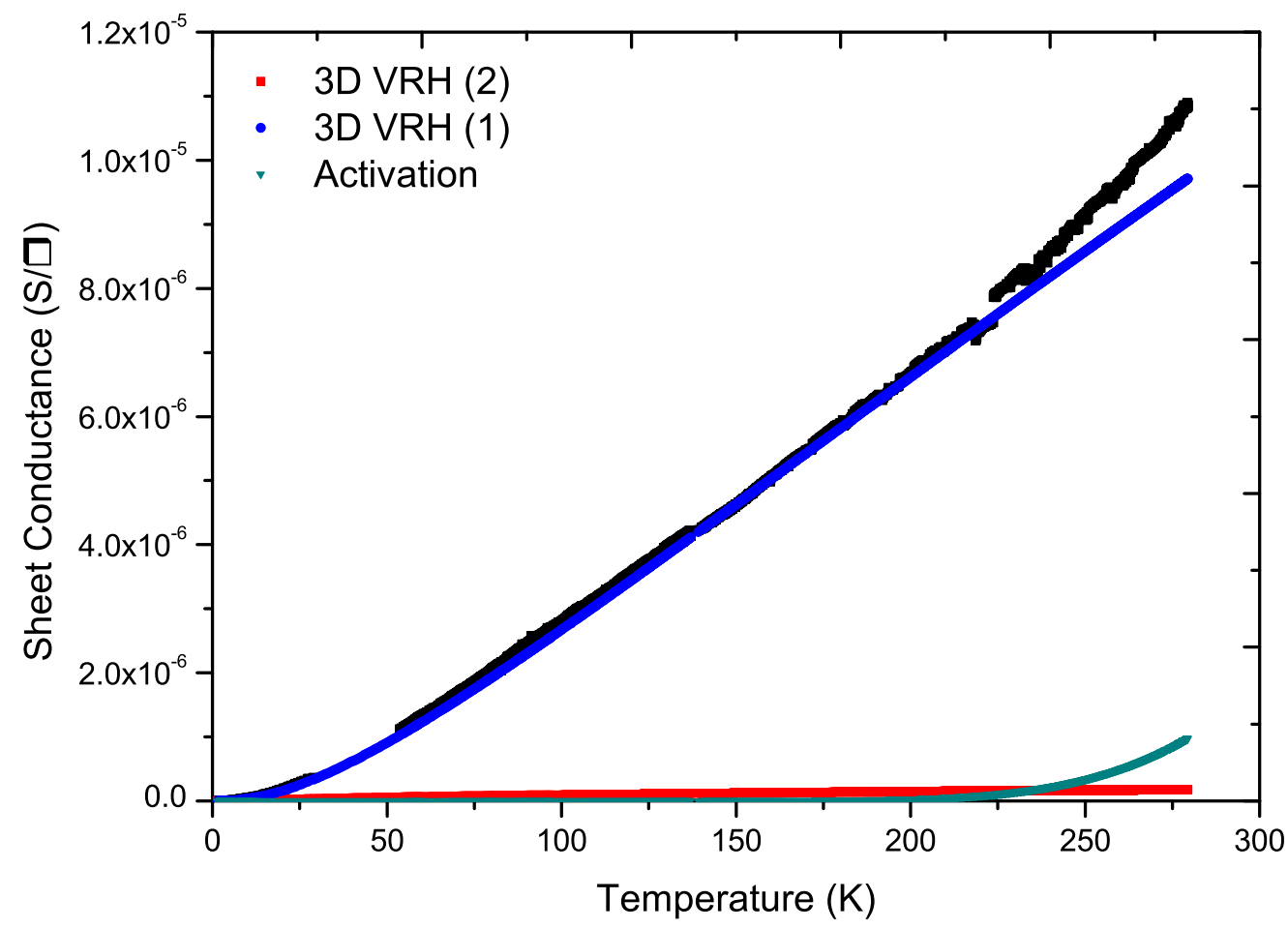

Figure 6.16: Conduction processes described by model 2 within s3. 
perature regime. From the inverse dependence of $T_{0}$ on the localisation length (Equation 2.17), we can see that the low temperature regime has a greater localisation length compared to the high temperature regime in both models. This could be explained by VRH conduction between a different set of localised states with a longer localisation length. While a residual of this mechanism exists to $300 \mathrm{~K}$, we expect localised states that are more closely spaced to dominate as the Fermi window widens. This results in a saturation conductance above 30 $\mathrm{K}$ (the blue curve in Figure 6.16). The exact nature and characteristic of the types of localised states within SWNT networks are still unknown and we cannot expound any further into the reason for this change-over in the localisation length.

Annealed samples follow 3D VRH though the shape of the conductance curve is that of sample with a lesser degree of localisation compared to U1. This is reflected in a $T_{0}$ value that is several orders of magnitude smaller than the unannealed film. The increase in the localisation length can be primarily attributed to the removal of residual amine due to accompanying improvements in the optoelectronic properties. However, other possibilities like the healing of defects after thermal treatment [78] or p-doping due to oxygen adsorption could also lead to the increase in the localisation length.

We have not included the effects of oxygen in the interpretation of our conduction data. It is well known that oxygen p-dopes semiconducting SWNTs $[108,117]$ which leads to an increase in sheet conductivity $[126,107,77]$. Several studies showed that oxygen can only be desorbed by exposure to UV radiation and high vacuum $[127,77]$ while the latter alone is insufficient to completely remove all the oxygen $[107,108]$. Conductance data for all the samples discussed in this chapter were obtained in a chamber evacuated to $\sim 10^{-6}$ mbar. However, we have no means of determining the extent of oxygen doping in our samples. 


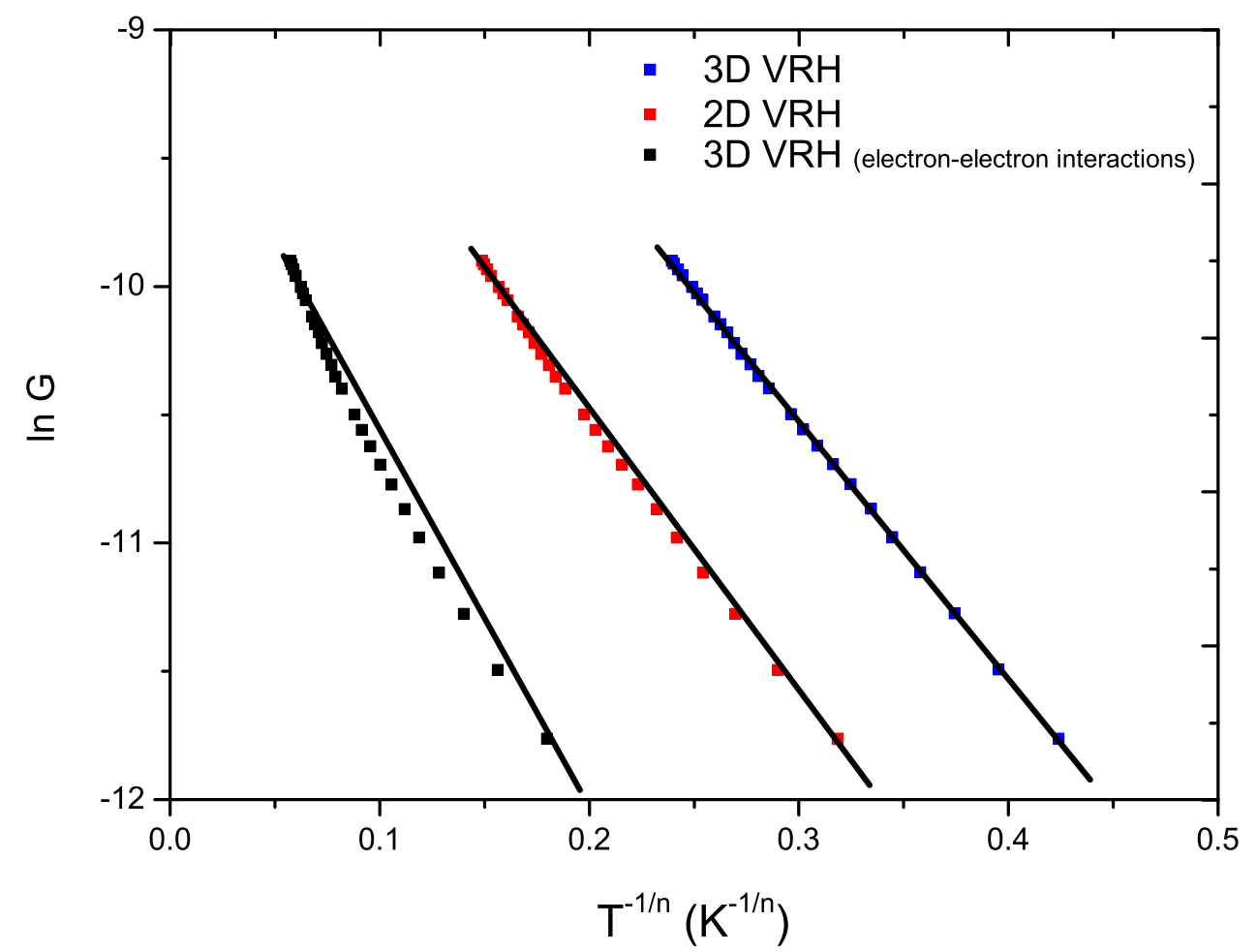

Figure 6.17: Temperature-dependent conductance curve for s4.

Thus, the reduction in $\mathrm{T}_{0}$ from the unannealed sample can also be attributed to p-doping from the adsorbed oxygen [77].

With the exception of $\mathrm{U} 2$, all the annealed and unannealed samples studied so far follow 3D VRH conduction. Figure 6.17 shows that even the annealed sample prepared from the same batch of butylamine as U3 (labelled s4) is dominated by 3D VRH. While s3 diverges from this mechanism at high temperatures, s4 rigorously follows 3D VRH to $300 \mathrm{~K}$ (parameters shown in Table 6.5). The conductance curve for s4 almost matches the unirradiated sample indicating that this sample has a larger localisation length than s3 (Figure 6.18).

While the intermediate to low temperature regions are dominated by VRH, activated transport begins to contribute significantly at high temperatures. Since this mechanism relies on phonon-activated conduction rather than field-driven conduction, no field-dependent behaviour should be observed. We can see this 


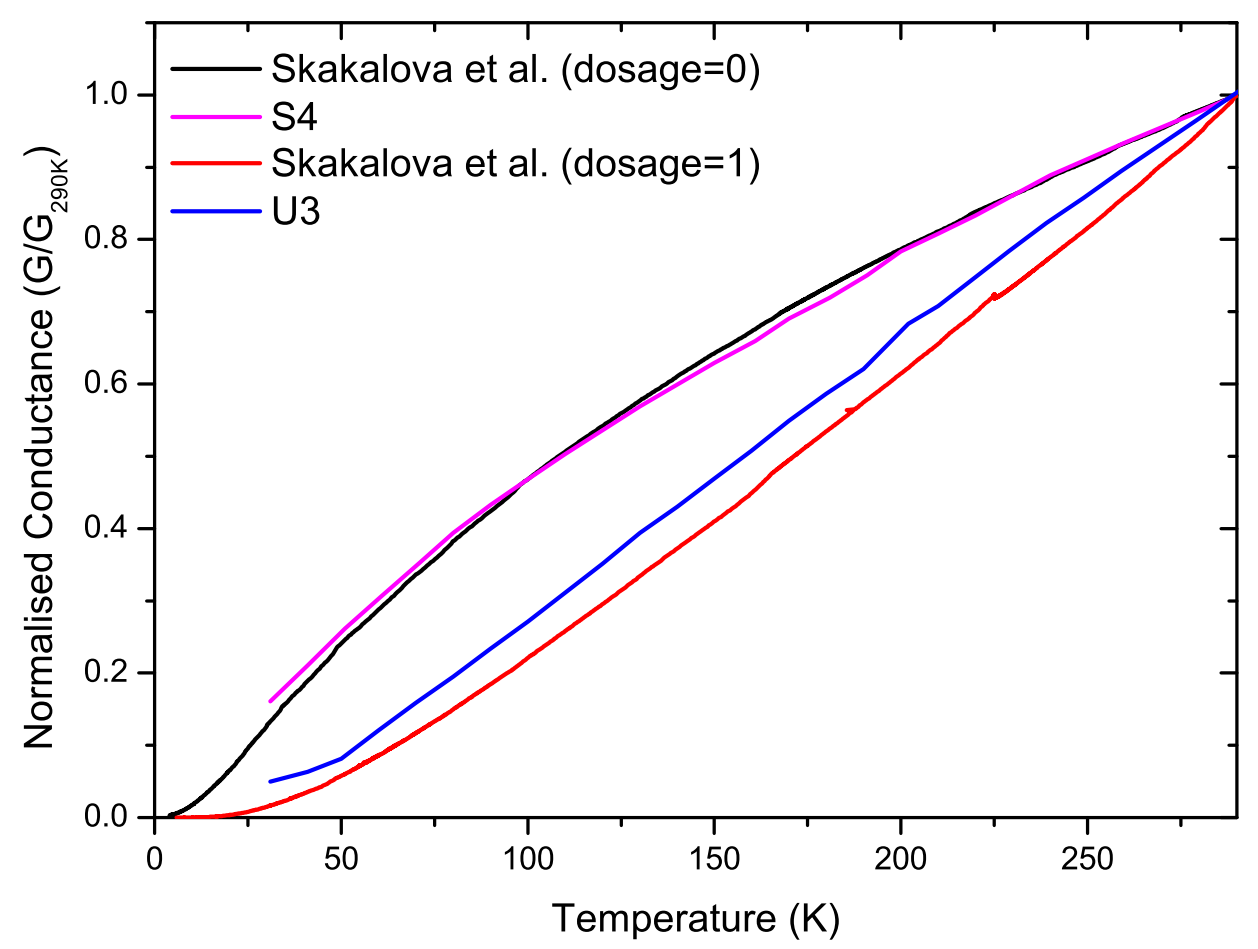

Figure 6.18: Normalised conductance for samples s4 and U3 compared to irradiated networks of Skakalova et al. [78]

\begin{tabular}{|l|l|l|}
\hline \multicolumn{3}{|c|}{ Model: $\mathrm{G}=\mathrm{G}_{0} \exp \left(\left(T_{0} / T\right)^{1 / 4}\right)$} \\
\hline & Annealed & Unannealed \\
\hline $\mathbf{G}_{0}$ & $5.6 \times 10^{-4}$ & $5.3 \times 10^{-4}$ \\
\hline $\mathbf{T}_{0}$ & 10490 & 97607 \\
\hline \hline
\end{tabular}

Table 6.5: Parameters from the 3D VRH fit for annealed and unannealed samples prepared during the initial stages of MSc.

in Figure 6.19 where the temperature-dependent form of the normalised conductance at $1 \mathrm{~V}$ is virtually identical to that at $50 \mathrm{~V}$. The four curves also come closer together above $200 \mathrm{~K}$. These characteristics of the annealed samples jointly support activation conduction at high temperatures.

The activation model gives an energy barrier equivalent to $200 \mathrm{meV}$ (Table 6.4). This value can be interpreted as the thermal excitation of electrons in armchair metallic tubes with pseudogaps. Other possibilities include the intertube barrier energy. Here, electrons are activated over inter-tube barriers when 


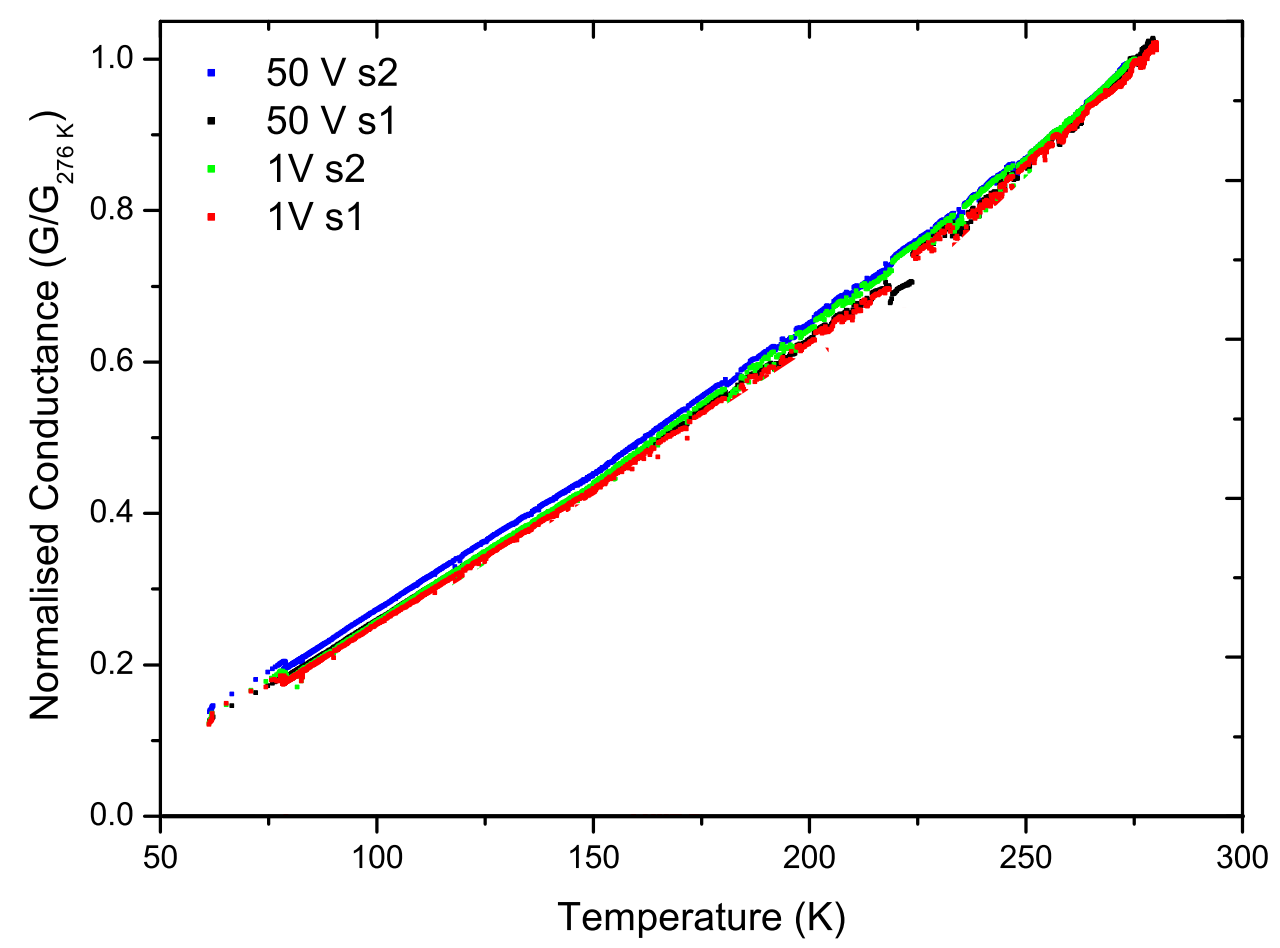

Figure 6.19: Normalised conductance vs. temperature for samples s1 and s2 at different applied voltages.

the thermal energy exceeds the barrier height instead of hopping between localised states within the barrier.

Armchair tubes are the only isolated SWNTs with a non-zero DOS at the Fermi level. However, the opening of a pseudogap within coherent bundles of the same chirality was theoretically predicted by many groups to the order of 100 $[28,128]-200 \mathrm{meV}[47,129]$. While this value closely resembles the barrier energy extracted from the fit, we are confident that there are no crystalline bundles within our SWNT networks. Monochiral bundles of armchair SWNTs are unlikely even in enriched metallic networks and our Raman results in Section 5.1.1 gave no indication of metallic selectivity.

On the other hand, Ouyang et al. recently presented experimental evidence of a pseudogap $(\sim 100 \mathrm{meV})$ in armchair tubes on the surface of multichiral ropes [27]. The proof of thermal excitation over such a gap would involve using a gate 


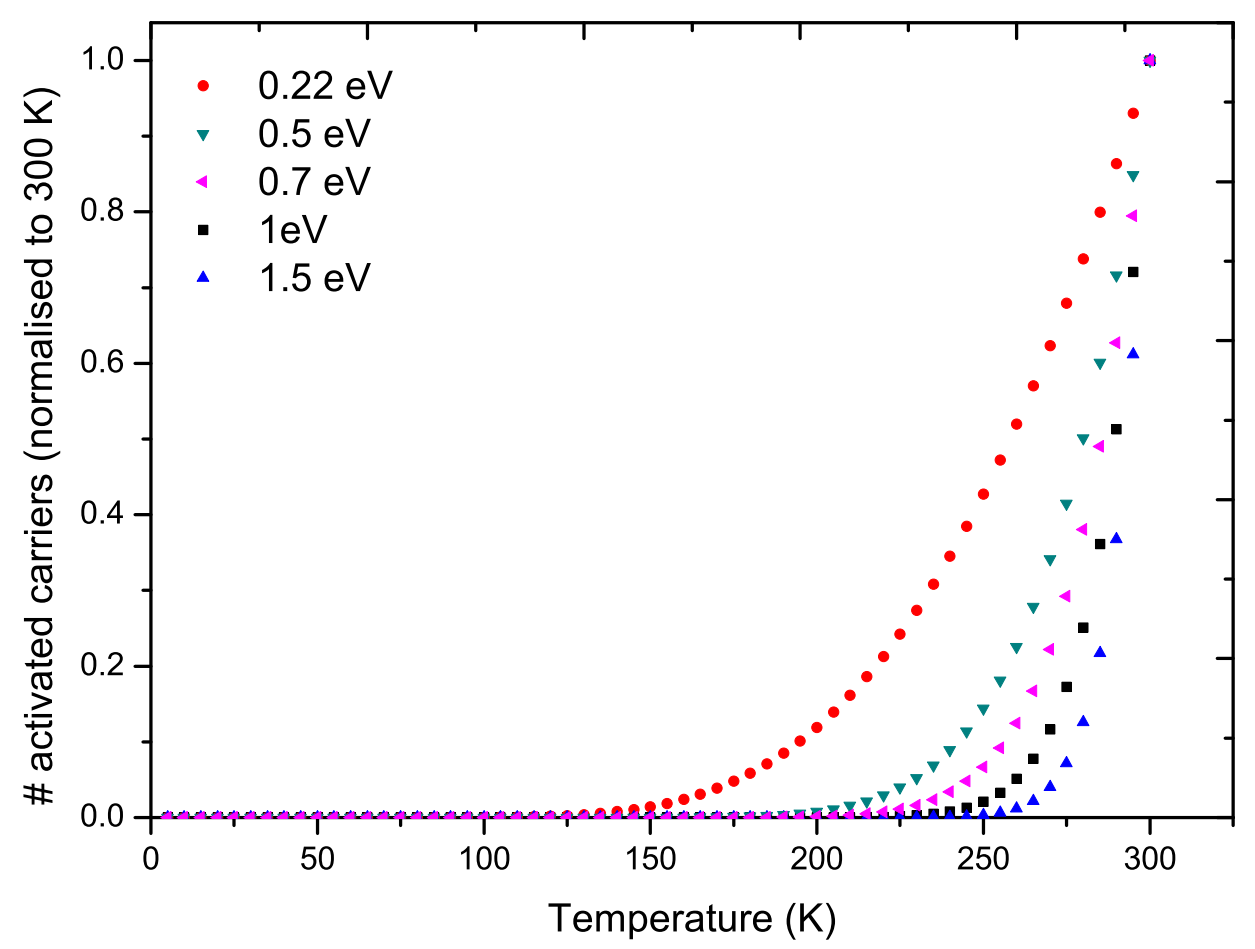

Figure 6.20: Plots of the number of carrier electrons at different bandgap energies from Equation 6.3.

voltage to 'turn off' semiconducting SWNTs [63] and observe the conductance as a function of temperature. A match with the curve for zero gate voltage would provide strong evidence for the activation of electrons over a pseudogap. Since this proof is beyond the scope of this study, we consider the alternative of activation over inter-tube barriers.

Activation over Schottky barriers (metallic-semiconducting tube junctions) with barrier heights between $200-300 \mathrm{meV}$ [65, 82] agrees with the activation energy obtained from our annealed sample. The barrier energy is approximately $\mathrm{E}_{G} / 2$ where $\mathrm{E}_{G}$ is the bandgap of the semiconductor [65].

The number of electrons in the conduction band of a conventional semiconductor as a function of temperature follows Boltzmann statistics and is given as: 


$$
N(\text { electrons in conduction band }) \sim \exp \left(-E_{G} / 2 k_{B} T\right)
$$

Figure 6.20 shows the 'turn-on' temperature for different bandgap energies. A bandgap of $700 \mathrm{meV}$ corresponds to a 'turn-on' temperature of $225 \mathrm{~K}$ which matches the picture of activation shown in Figure 6.16 which turns on at $\sim 220$ K. Nanotubes with diameter $\sim 14 \AA$ have a bandgap around $700 \mathrm{meV}$ [130]. This diameter falls within the range expected from HiPCO tubes: $7 \AA-14 \AA$ [131]. However, bundling-induced downshifts (up to $160 \mathrm{meV}$ [30]) bring the bandgap energies further down to $\sim 900 \mathrm{meV}$ corresponding to a diameter of $\sim 10 \AA$ : the mean diameter expected for HiPCO nanotubes [131].

We suggest that the high temperature conduction regime is due to thermal activation of electrons over metal-semiconductor junctions. This mechanism is supported by the mixed nature of percolating pathways in thin networks (Section 2.3.3) since they contain both metallic and semiconducting tubes. However, we have insufficient evidence to advance this possibility over that of thermal activation in bundled armchair tubes.

\subsection{Conclusion}

3D VRH conduction is the mechanism responsible for conduction in both annealed and unannealed networks. The relative value of the $T_{0}$ parameter was used to gauge the extent of localisation between different samples.

The large amount of surfactant residue in unannealed samples was found to hamper the electron transport in unannealed samples compared to annealed samples. However, the reduction in $\mathrm{T}_{0}$ and improvements to optoelectronic properties 
upon annealing may not be solely due to surfactant removal. Physical effects such as oxygen-induced p-doping and healing of defects upon annealing can also affect the $\mathrm{T}_{0}$ parameter.

Four parallel processes were invoked to fully explain the full set of conduction data obtained from the annealed films: a low temperature tunnelling current, low temperature VRH, 3D VRH conduction between $30 \mathrm{~K}-200 \mathrm{~K}$ and activation conduction above $200 \mathrm{~K}$. The low temperature VRH is due to a subset of localised states with a larger localisation length but the nature of these states is unknown. When these localised states freeze out at very low temperatures, a small tunnelling current is observed to $\sim 1.3 \mathrm{~K}$. The high temperature activation conduction is the result of thermal activation over inter-tube barriers or thermal excitation of electrons over the pseudogap within the semi-metallic armchair tubes. 


\section{Chapter 7}

\section{Conclusion}

An effective SWNT dispersion requires a surfactant that interacts with both the SWNT and the solvent. Butylamine adsorbs on the nanotube sidewall with a binding energy of $45 \mathrm{meV}$ and remains bound until it is desorbed by annealing. Butylamine and THF are miscible solvents since their density, CED and polarity are very similar in magnitude. The amine and THF interact via hydrogen bonding where the hydrogens on the amino group form weak covalent bonds with the oxygen on the THF molecule. Thus, nanotube dispersions prepared with this solvent mixture are stable for months.
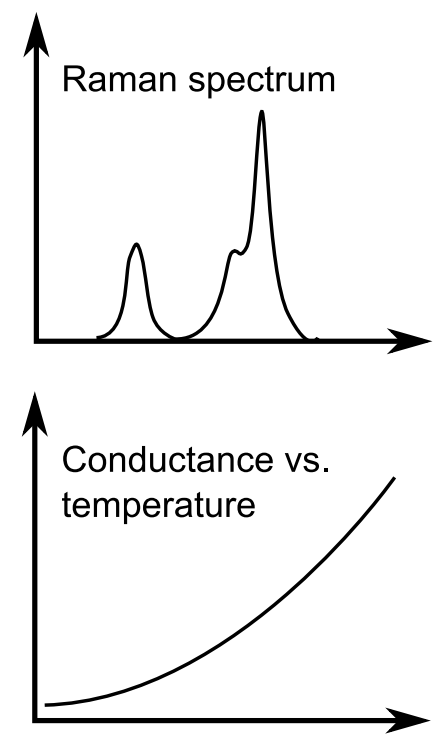

Figure 7.1: General summary of results presented in Chapters 5 and 6.

\section{Frequency shift $\longrightarrow$ extent of bundling \& Resonance window \\ 2. Integrated area $\longrightarrow$ electronic selectivity \\ 3. Observed RBMs $\rightarrow$ effects of combustion}

\section{1. $T_{0}$ parameter $\longrightarrow$ localisation length \\ 2. Shape of $\longrightarrow$ extent of localisation conductance curve}


The transformation of the bundled raw powder to an unbundled SWNT dispersion was also accompanied by a change in the resonance window. RBMs observed in the centrifuged dispersions were found within a resonance window of $115 \mathrm{meV}$. The resonance window for the raw powder was estimated to be 220 meV. This reduction in the resonance window was shown by several groups to support the case for unbundling $[58,57]$. However, this resonance window is only crude estimate. A more precise gauge for the extent of bundling was determined from the frequency shift of the RBMs.

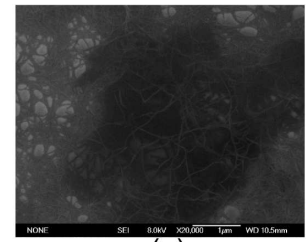

(a)

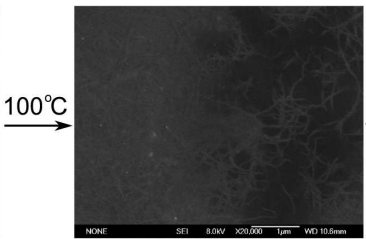

(b)

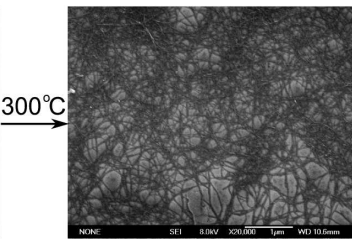

(c)

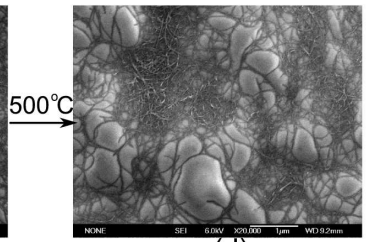

(d)

Figure 7.2: SEM images of SWNT films annealed at various temperatures taken with a magnification of $20,000 \times$. (a) An unannealed network. The dark patches depict pyrolysed butylamine and THF residue. (b) A SWNT network annealed at $100{ }^{\circ} \mathrm{C}$. The dark patch indicates the presence of adsorbed butylamine. (c) SWNT network annealed to $300{ }^{\circ} \mathrm{C}$. The removal of adsorbed butylamine is reflected in the morphology. (d) SWNT network annealed at $500{ }^{\circ} \mathrm{C}$. Many nanotubes have combusted during this anneal leaving behind a sparser network.

An upshift of $\sim 3 \mathrm{~cm}^{-1}$ was found in in the RBM frequencies of well-exfoliated solutions compared to the raw powder. Effects of bundling-induced downshifts in Raman frequency were also observed for the G-band in annealed films. Both the $\mathrm{G}^{+}$and $\mathrm{G}^{-}$modes also showed a decrease in frequency in spots containing large clumps of nanotubes. Hence, we speculate that the frequency redshift arises due to delocalisation of electrons in nanotube bundles. The delocalisation would lengthen the $\mathrm{C}-\mathrm{C}$ bond and soften the vibrational frequency. This characteristic shows promise as a tool for determining bundle sizes in SWNT samples from the magnitude of the frequency shift. 


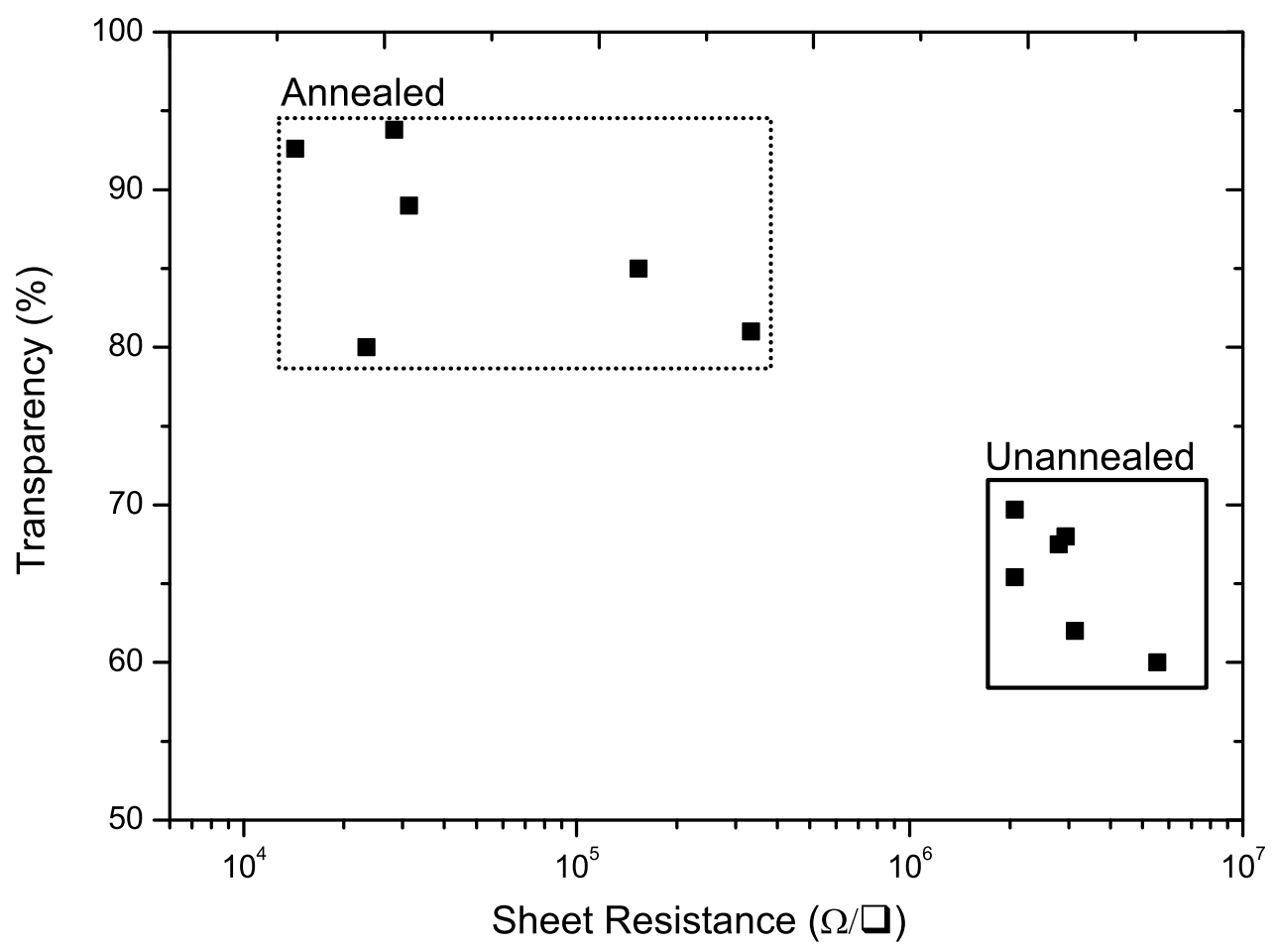

Figure 7.3: Effects of annealing on optoelectronic properties.

As-prepared films were found to contain residual solvent and surfactant. Figure 7.2 (a) shows an unannealed film with a significant amount of solvent residue. Annealing the film at $100{ }^{\circ} \mathrm{C}$ removes the loose solvent though the adsorbed butylamine remains bound to the nanotube sidewall as shown in Figure 7.2 (b). Figure 7.2 (c) shows a change in film morphology after annealing at $300{ }^{\circ} \mathrm{C}$. The annealing removes all the adsorbed butylamine and the SWNT network sits tightly against the substrate.

As shown in Figure 7.3, SWNT films fabricated from butylamine dispersions show considerable improvement in optoelectronic properties upon annealing. Annealing films at $300{ }^{\circ} \mathrm{C}$ was found to decrease sheet resistance by at least two orders of magnitude and improve optical transparency from $\sim 60 \%$ to above 80 $\%$. 
Previous studies of SWNTs dispersed in alkylamine and THF reported enrichment for metallic nanotubes $[18,19]$. However, our results indicate a nonselective process. The ratio of metallic to semiconducting tubes in the centrifuged dispersion was unchanged relative to that of the sonicated dispersion. This nonselectivity means that despite their high optical transparency, our SWNT networks cannot attain low sheet resistances. Thus, the limiting factor preventing significant improvements to conductivity is the low ratio of metallic to semiconducting tubes. The number of highly conducting, metallic paths are few so the electrons traverse paths with lower conductivity thus bringing down the sheet conductivity.

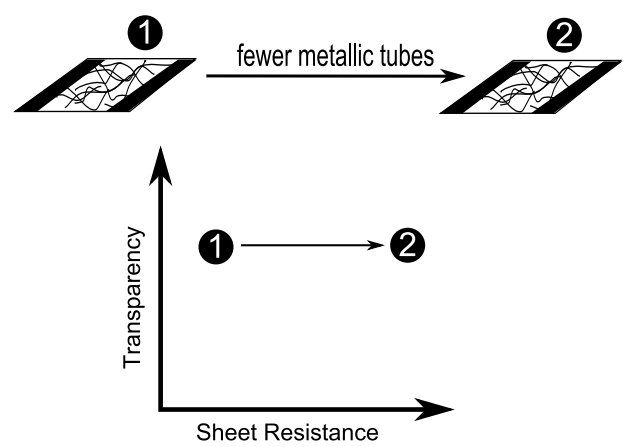

Figure 7.4: Change in sheet resistance for the same optical transparency in films enriched in metallic SWNTs.

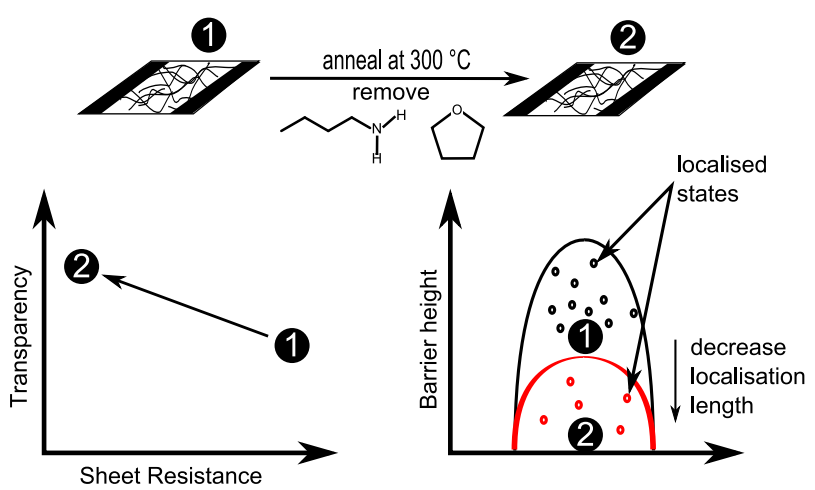

Figure 7.5: Increase in optoelectronic properties and decrease in localisation length upon annealing.

Conduction studies on unannealed and annealed films revealed that both film 
types follow 3D variable range hopping. In these films, electron transport is hindered by large inter-tube barriers. Hence, electrons conduct by hopping between localised states. The localisation length was qualitatively assesed from the $T_{0}$ parameter. The relative value of this parameter was considerably reduced in annealed films. This change was associated with the loss of large inter-tube barriers. A high temperature activation regime was observed in annealed samples. An activation energy of $200 \mathrm{meV}$ could be associated with thermal activation over Schottky barriers or activation of electrons within armchair tubes.

The annealing treatment was also found to oxidise nanotubes at temperatures as low as $300{ }^{\circ} \mathrm{C}$. Annealing to $500{ }^{\circ} \mathrm{C}$ preferentially combusted tubes with high chiral angle and small diameter. Figure 7.2 (d) shows a SWNT film annealed at $500{ }^{\circ} \mathrm{C}$. The coverage is patchy and more of the rough surface of the glass is exposed indicating considerable oxidation. With more study, this technique can be developed as a tool for reducing the chiral population of nanotubes in a given sample. 


\section{Appendix A}

\section{LabView program for closed-cycle cryostat}

The front panel of the virtual instrument (VI) used to control the temperature and acquire conductivity data is shown in Figure A.1. The program was written in LabView by Andrew Preston (PhD student at VUW). The main function of the VI was to call the required subroutines: setting the target temperature, performing the I-V sweep and saving the data. The routines are described in order in the following sections.

\section{Reaching the target temperature}

The various target temperatures were entered into the expandable array on the left hand side of the front panel. The allowed error in the final temperature and the time for stability were specified $\pm 1 \mathrm{~K}$ and $1 \mathrm{~s}$ respectively before each run. These parameters enabled the user to control the accuracy and stability of the temperature at which the measurements were taken. This first subroutine called by the main VI essentially took the target temperature, error and stability time and accordingly set the PID parameters of the temperature controller. The next 
subroutine was triggered once the target temperature was stable for the specified time within the uncertainty as shown in Figure A.2.

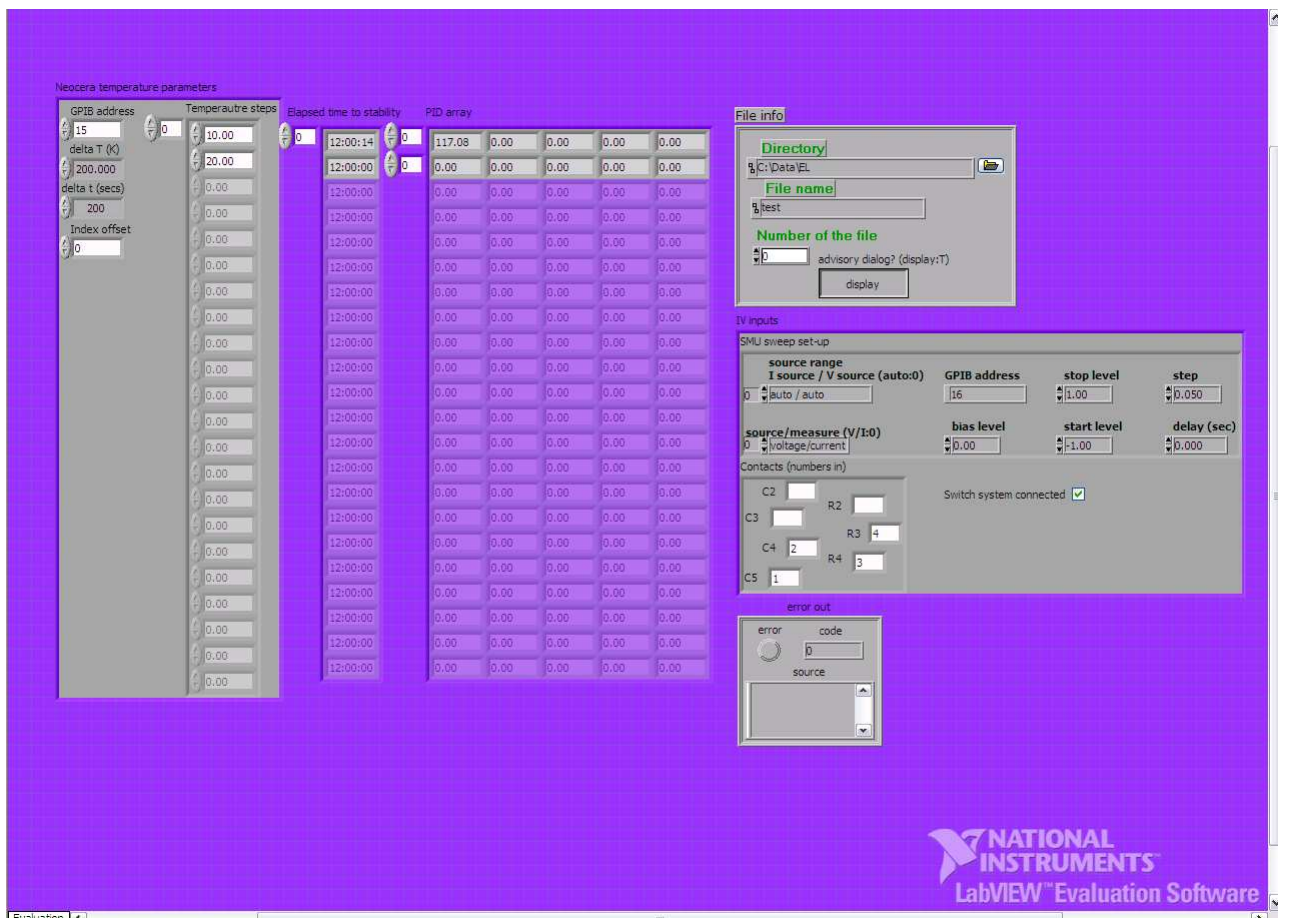

Figure A.1: Front panel screenshot of the LabView program used to set the target temperature.

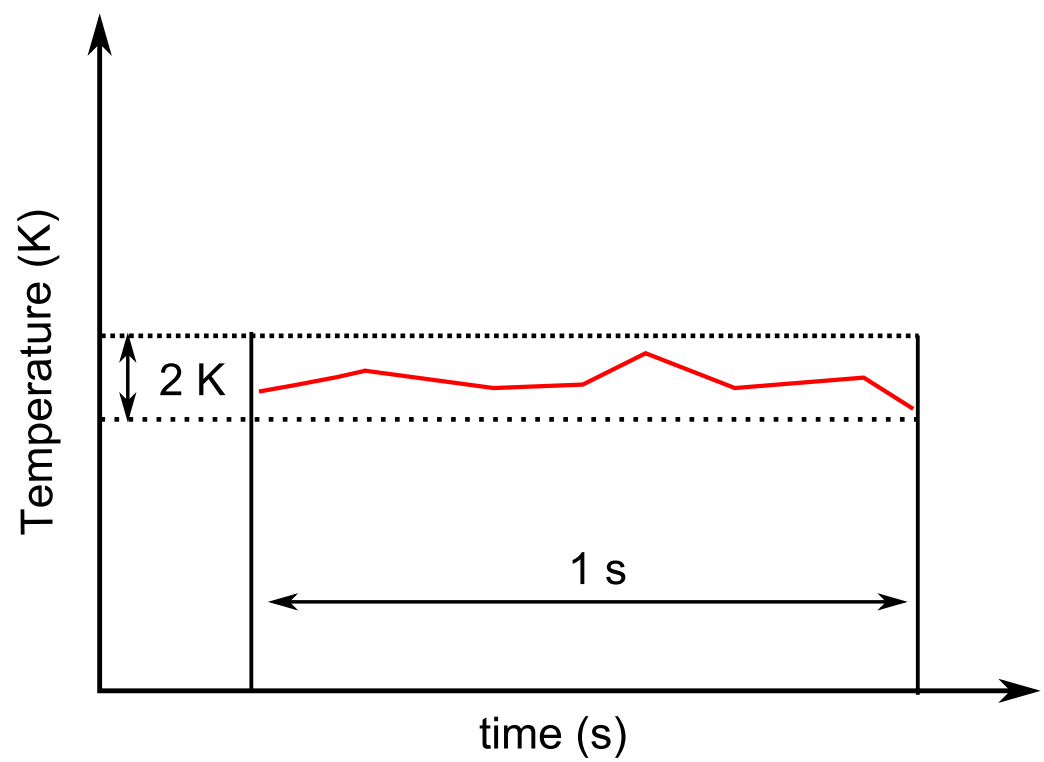

Figure A.2: The plot of temperature vs. time displayed in the 'time to stability' subroutine. 


\section{Performing the I-V sweep}

The parameters of the I-V sweep subroutine were set on the right hand side of the main VI. This routine was specific to the Keithley 236 SMU. The following sweep parameters were set:

1. Source: voltage. Measure: current

2. Voltage sweep from $-100 \mathrm{~V}$ to $100 \mathrm{~V}$

3. Voltage step of $10 \mathrm{~V}$

\section{Saving the data}

Once the I-V sweep was performed, the final subroutine saved the sweep data to a file. The target temperature was appended to the file name.

The three subroutines continued in a loop until the last target temperature. Two runs were performed for each sample. The first was a ramp down in temperature where the values were set from high to low. The second was the ramp up with low values set at the top of the table. 


\section{Appendix B}

\section{LabView program for glass \\ cryostat}

As discussed in Section 3.4.2, the samples were connected in parallel. The Keithley $236 \mathrm{SMU}$ supplied a constant voltage $(50 \mathrm{~V}$ or $\pm 50 \mathrm{~V}$ and $\pm 1 \mathrm{~V})$ to each sample. The current across the sample was measured by a digital multimeter connected in series. The LabView program simply polled the current reading from each multimeter and stored that in a file.

\section{Polling measurements}

As shown in Figure B.1, the measurement from the Rh-Fe resistor and samples were polled serially. For each sample, the current measurement and the voltage reading of the thermometer were recorded as a $2 \mathrm{D}$ array. The first set of measurements was saved under a file and all subsequent measurements were appended to this file. This polling and saving process was repeated for the remaining multimeters. Each multimeter was polled once in the loop which continued until the program was manually stopped (the large green button in Figure B.2). 


\section{Waveform charts}

The polled readings from the multimeters were fed into waveform charts as shown on the far right in Figure B.2. This was done for a visual monitoring of temper-
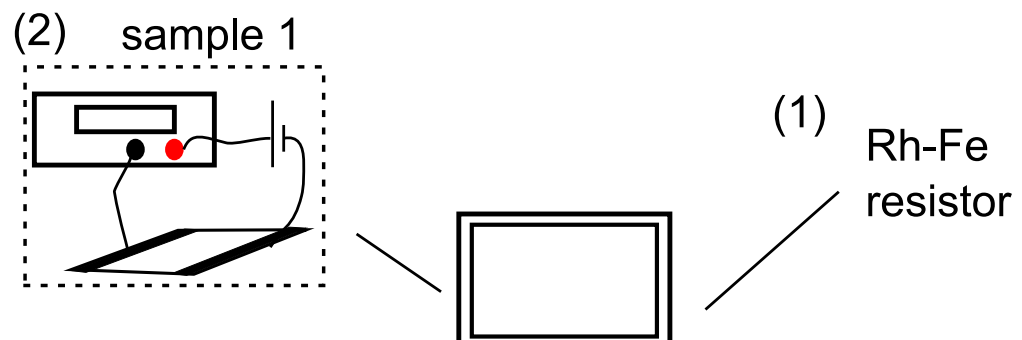

(3) sample 2
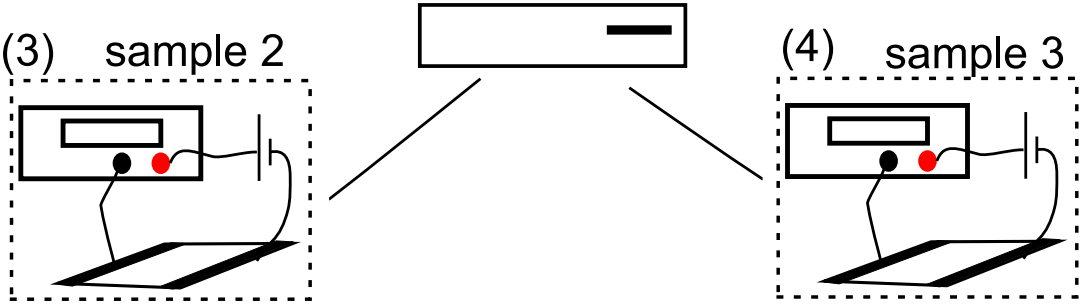

Figure B.1: Serial polling of the four digital multimeters. The number in the brackets indicate the order of the poll.

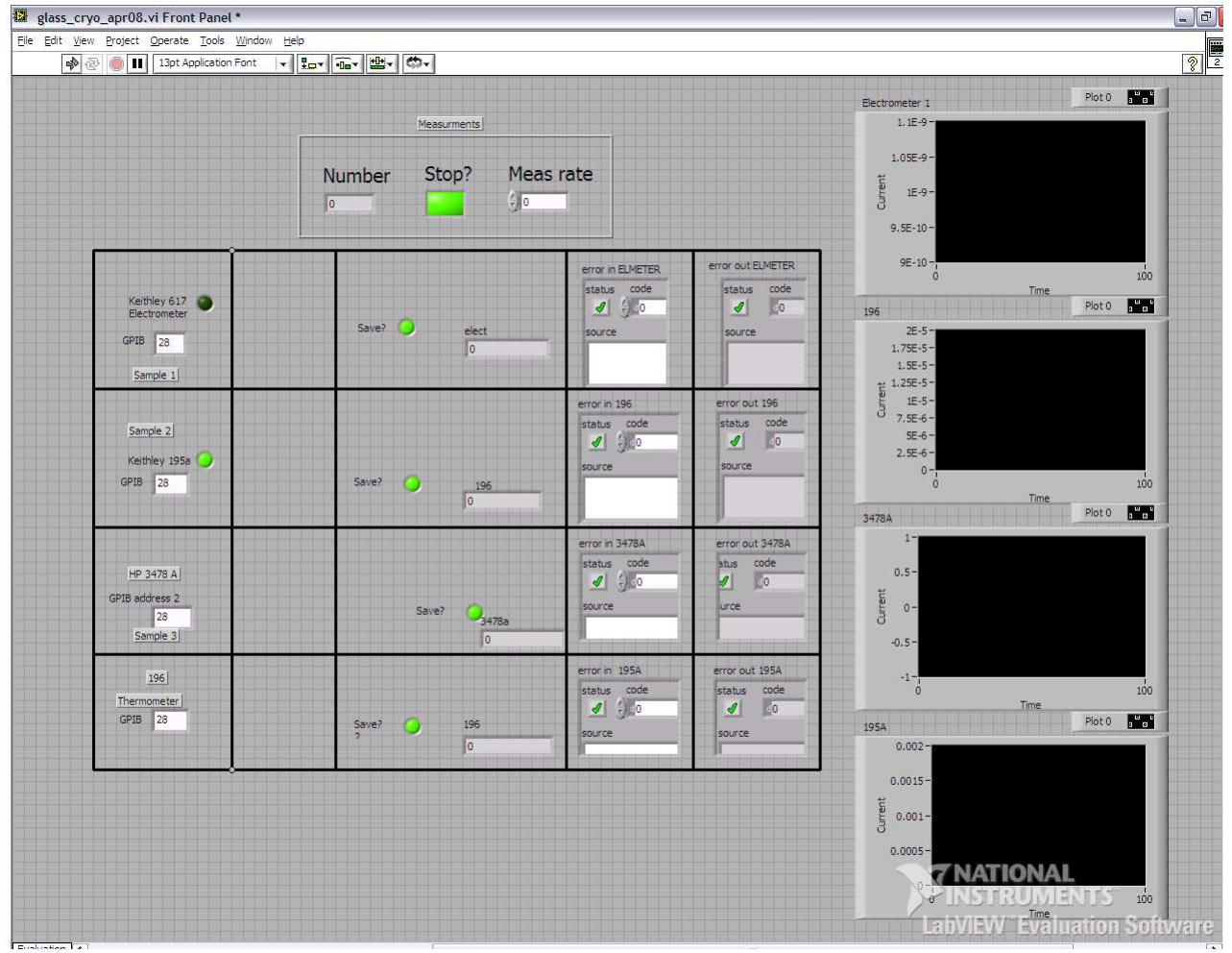

Figure B.2: Front panel screenshot of the LabView program which triggered the four connected DMMs. The waveform charts plotted the readings from each DMM as a function of measurement number. 
ature readings and the sample current. 


\section{References}

[1] S. Iijima, Nature 354, 56 (1991).

[2] L. V. Radushkevich and V. M. Lukyanovich, Russian Journal of Physical Chemistry 26, 88 (1952).

[3] M. Monthioux and V. L. Kuznetsov, Carbon 44, 1621 (2006).

[4] B. Q. Wei, R. Vajtai, and P. M. Ajayan, Applied Physics Letters 79, 1172 (2001).

[5] M. F. Yu, B. S. Files, S. Arepalli, and R. S. Ruoff, Physical Review Letters 84, 5552 (2000).

[6] Y. H. Yoon, J. W. Song, D. Kim, J. Kim, J. K. Park, S. K. Oh, and C. S. Han, Advanced Materials 19, 4284 (2007).

[7] T. Takenobu, T. Takahashi, T. Kanbara, K. Tsukagoshi, Y. Aoyagi, and Y. Iwasa, Applied Physics Letters 88, 033511 (2006).

[8] D. Zhang, K. Ryu, X. Liu, E. Polikarpov, J. Ly, M. E. Tompson, and C. Zhou, Nano Letters 6, 1880 (2006).

[9] A. Du Pasquier, H. E. Unalan, A. Kanwal, S. Miller, and M. Chhowalla, Applied Physics Letters 87, 203511 (2005). 
[10] M. W. Rowell, M. A. Topinka, M. D. McGehee, H. J. Prall, G. Dennler, N. S. Sariciftci, L. Hu, and G. Gruner, Applied Physics Letters 88, 233506 (2006).

[11] http://www.gizmag.com/samsung-demontrates-color-carbon-nanotubebased-electrophoretic display/10220/, Samsung demontrates color carbon nanotube-based electrophoretic display, 2008.

[12] T. Mustonen, J. Mäklin, K. Kordás, N. Halonen, G. Tóth, S. Saukko, J. Vähäkangas, H. Jantunen, S. Kar, P. M. Ajayan, R. Vajtai, H. Helistö, P. Heikki Seppä, and H. Moilanen, Physical Review B 77, 125430 (2008).

[13] B. Gao, G. Z. Yue, Q. Qiu, Y. Cheng, H. Shimoda, L. Fleming, and O. Zhou, Advanced Materials 13, 1770 (2001).

[14] R. Krupke, S. Linden, M. Rapp, and F. Hennrich, Advanced Materi$\operatorname{als}(\mathrm{FRG}) \mathbf{1 8}, 1468$ (2006).

[15] N. Choi, M. Kimura, H. Kataura, S. Suzuki, Y. Achiba, W. Mizutani, and H. Tokumoto, Japanese Journal of Applied Physics 41, 6264 (2002).

[16] G. G. Samsonidze, S. G. Chou, A. P. Santos, V. W. Brar, G. Dresselhaus, M. S. Dresselhaus, A. Selbst, A. K. Swan, M. S. Ünlü, B. B. Goldberg, D. Chattopadhyay, S. N. Kim, and F. Papadimitrakopoulos, Applied Physics Letters 85, 1006 (2004).

[17] Y. Maeda, S. Kimura, Y. Hirashima, M. Kanda, Y. Lian, T. Wakahara, T. Akasaka, T. Hasegawa, H. Tokumoto, T. Shimizu, H. Kataura, Y. Miyauchi, S. Maruyama, K. Kobayashi, and S. Nagase, Journal of Physical Chemistry B 108, 18395 (2004).

[18] Y. Maeda, S. Kimura, M. Kanda, Y. Hirashima, T. Hasegawa, T. Wakahara, Y. Lian, T. Nakahodo, T. Tsuchiya, T. Akasaka, X. Lu, J. Zhang, Z. Gao, 
Y. Yu, S. Nagase, S. Kazaoui, N. Minami, T. Shimizu, H. Tokumoto, and R. Saito, Journal of the American Chemical Society 127, 10287 (2005).

[19] Y. Maeda, Y. Takano, A. Sagara, M. Hashimoto, M. Kanda, S. Kimura, Y. Lian, T. Nakahodo, T. Tsuchiya, T. Wakahara, T. Akasaka, T. Hasegawa, S. Kazaoui, N. Minami, J. Lu, and S. Nagase, Carbon 46, $1563(2008)$.

[20] Y. Maeda, M. Hashimoto, S. Kaneko, M. Kanda, T. Hasegawa, T. Tsuchiya, T. Akasaka, Y. Naitoh, T. Shimizu, H. Tokumoto, J. Lu, S. Nagase, M. S. Ünlü, B. B. Goldberg, and R. Saito, Journal of Materials Chemistry 18, 4189 (2008).

[21] Y. Miyata, K. Yanagi, Y. Maniwa, and H. Kataura, J. Phys. Chem. C 112, 3591 (2008).

[22] B. Parekh, G. Fanchini, G. Eda, and M. Chhowalla, Applied Physics Letters 90, $121913(2007)$.

[23] A. Schindler, J. Brill, N. Fruehauf, J. P. Novak, and Z. Yaniv, Physica E 37, 119 (2007).

[24] C. T. White and J. W. Mintmire, Journal of Physical Chemistry B 109, $52(2005)$.

[25] E. Richter and K. R. Subbaswamy, Physical Review Letters 79, 2738 (1997).

[26] S. Reich, C. Thomsen, and J. Maultzsch, Carbon Nanotubes: Basic Concepts and Physical Properties, Wiley-VCH, 2004.

[27] M. Ouyang, J. L. Huang, C. L. Cheung, and C. M. Lieber, Science 292, $702(2001)$.

[28] P. Delaney, H. Joon Choi, J. Ihm, S. G. Louie, and M. L. Cohen, Physical Review B 60, 7899 (1999). 
[29] C. Fantini, A. Jorio, M. Souza, M. S. Strano, M. S. Dresselhaus, and M. A. Pimenta, Physical Review Letters 93, 147406 (2004).

[30] M. J. O’Connell, S. Sivaram, and S. K. Doorn, Physical Review B 69, $235415(2004)$.

[31] A. B. Kaiser, V. Skákalová, and S. Roth, Physica E 40, 2311 (2007).

[32] C. V. Raman and K. S. Krishnan, Nature 121, 501 (1928).

[33] C. V. Raman and K. S. Krishnan, Nature 121, 711 (1928).

[34] J. R. Ferraro, K. Nakamoto, and C. W. Brown, Introductory Raman Spectroscopy, Academic Press, 2 edition, 2003.

[35] S. Piscanec, M. Lazzeri, J. Robertson, A. C. Ferrari, and F. Mauri, Physical Review B 75, 35427 (2007).

[36] A. Jorio, G. Dresselhaus, M. S. Dresselhaus, M. Souza, M. S. S. Dantas, M. A. Pimenta, A. M. Rao, R. Saito, C. Liu, and H. M. Cheng, Physical Review Letters 85, 2617 (2000).

[37] A. Jorio, A. G. S. Filho, G. Dresselhaus, M. S. Dresselhaus, A. K. Swan, M. S. Ünlü, B. B. Goldberg, M. A. Pimenta, J. H. Hafner, C. M. Lieber, et al., Physical Review B 65, 155412 (2002).

[38] A. Jorio, M. A. Pimenta, A. G. S. Filho, R. Saito, G. Dresselhaus, and M. S. Dresselhaus, New Journal of Physics 5, 139 (2003).

[39] S. Brown, A. Jorio, P. Corio, M. S. Dresselhaus, G. Dresselhaus, R. Saito, and K. Kneipp, Physical Review B 63, 155414 (2001).

[40] K. T. Nguyen, A. Gaur, and M. Shim, Physical Review Letters 98, 145504 (2007). 
[41] M. S. Dresselhaus, G. Dresselhaus, A. Jorio, A. G. S. Filho, and R. Saito, Carbon 40, 2043 (2002).

[42] J. Maultzsch, S. Reich, and C. Thomsen, Physical Review B 64, 121407 (2001).

[43] H. Kataura, Y. Kumazawa, Y. Maniwa, I. Umezu, S. Suzuki, Y. Ohtsuka, and Y. Achiba, Synthetic Metals 103, 2555 (1999).

[44] A. Jorio, R. Saito, J. H. Hafner, C. M. Lieber, M. Hunter, T. McClure, G. Dresselhaus, and M. S. Dresselhaus, Physical Review Letters 86, 1118 (2001).

[45] L. Henrard, E. Hernández, P. Bernier, and A. Rubio, Physical Review B 60, 8521 (1999).

[46] M. Milnera, J. Kürti, M. Hulman, and H. Kuzmany, Physical Review Letters 84, 1324 (2000).

[47] A. M. Rao, J. Chen, E. Richter, U. Schlecht, P. C. Eklund, R. C. Haddon, U. D. Venkateswaran, Y. K. Kwon, and D. Tománek, Physical Review Letters 86, 3895 (2001).

[48] G. S. Duesberg, I. Loa, M. Burghard, K. Syassen, and S. Roth, Physical Review Letters 85, 130 (2000).

[49] J. Maultzsch, H. Telg, S. Reich, and C. Thomsen, Physical Review B 72, $205438(2005)$.

[50] T. Murakami, K. Kisoda, T. Tokuda, K. Matsumoto, H. Harima, K. Mitikami, and T. Isshiki, Diamond \& Related Materials 16, 1192 (2007).

[51] N. Izard, D. Riehl, and E. Anglaret, Physical Review B 71, 195417 (2005). 
[52] J. Sandler, M. S. P. Shaffer, A. H. Windle, M. P. Halsall, M. A. MontesMorán, C. A. Cooper, and R. J. Young, Physical Review B 67, 35417 (2003).

[53] U. D. Venkateswaran, A. M. Rao, E. Richter, M. Menon, A. Rinzler, R. E. Smalley, and P. C. Eklund, Physical Review B 59, 10928 (1999).

[54] V. Skakalova, A. B. Kaiser, U. Dettlaff-Weglikowska, K. Hrncarikova, and S. Roth, J Phys Chem B 109, 7174 (2005).

[55] E. B. Barros, A. G. S. Filho, V. Lemos, J. M. Filho, S. B. Fagan, M. H. Herbst, J. M. Rosolen, C. A. Luengo, and J. G. Huber, Carbon 43, 2495 (2005).

[56] J. R. Wood and H. D. Wagner, Applied Physics Letters 76, 2883 (2000).

[57] D. A. Heller, P. W. Barone, J. P. Swanson, R. M. Mayrhofer, and M. S. Strano, Journal of Physical Chemistry B 108, 6905 (2004).

[58] L. M. Ericson and P. E. Pehrsson, Journal of Physical Chemistry B 109, 20276 (2005).

[59] S. Reich, C. Thomsen, and P. Ordejón, Physical Review B 65, 155411 (2002).

[60] F. Hennrich, R. Krupke, S. Lebedkin, K. Arnold, R. Fischer, D. E. Resasco, and M. M. Kappes, Journal of Physical Chemistry B 109, 10567 (2005).

[61] L. Hu, D. S. Hecht, and G. Grüner, Nano Letters 4, 2513 (2004).

[62] E. S. Snow, J. P. Novak, P. M. Campbell, and D. Park, Applied Physics Letters 82, 2145 (2003). 
[63] M. Stadermann, S. J. Papadakis, M. R. Falvo, J. Novak, E. S. Snow, Q. Fu, J. Liu, Y. Fridman, J. J. Boland, R. Superfine, and S. Washburn, Physical Review B 69, 201402 (2004).

[64] D. Stauffer and A. Aharony, Introduction to Percolation Theory, CRC Press, 1994.

[65] M. Fuhrer, J. Nygård, L. Shih, M. Forero, Y. Yoon, M. Mazzoni, H. Choi, J. Ihm, S. Louie, A. Zettl, and P. L. McEuen, Science 288, 494 (2000).

[66] D. S. Hecht, L. Hu, and G. Grüner, Applied Physics Letters 89, 133112 (2006).

[67] K. L. Lu, R. M. Lago, Y. K. Chen, M. L. H. Green, P. J. F. Harris, and S. C. Tsang, Carbon 34, 814 (1996).

[68] M. Monthioux, B. Smith, B. Burteaux, A. Claye, J. Fischer, and D. Luzzi, Carbon 39, 1251 (2001).

[69] A. B. Kaiser, G. Düsberg, and S. Roth, Physical Review B 57, 1418 (1998).

[70] P. Sheng, Physical Review B 21, 2180 (1980).

[71] N. F. Mott, Philosophical Magazine 19 (1969).

[72] Z. Li, H. R. Kandel, E. Dervishi, V. Saini, A. S. Biris, A. R. Biris, and D. Lupu, Applied Physics Letters 91, 053115 (2007).

[73] P. W. Anderson, Physical Review 109, 1492 (1958).

[74] N. F. Mott, Philosophical Magazine 17, 1259 (1968).

[75] R. Zallen, The physics of amorphous solids, John Wiley \& Sons New York, 1983. 
[76] V. Ambegaokar, B. I. Halperin, and J. S. Langer, Physical Review B 4, $2612(1971)$.

[77] C. Morgan, Z. Alemipour, and M. Baxendale, Physica Status Solidi (a) 205, 1394 (2008).

[78] V. Skákalová, A. B. Kaiser, Z. Osváth, G. Vértesy, L. P. Biró, and S. Roth, Applied Physics A 90, 597 (2008).

[79] V. Skákalová, A. Kaiser, Y. Woo, and S. Roth, Physical Review B 74, 85403 (2006).

[80] D. P. Wang, D. E. Feldman, B. R. Perkins, A. J. Yin, G. H. Wang, J. M. Xu, and A. Zaslavsky, Solid State Communications 142, 287 (2007).

[81] O. Hilt, H. B. Brom, and M. Ahlskog, Physical Review B 61, 5129 (2000).

[82] D. H. Kim, J. Huang, H. K. Shin, S. Roy, and W. Choi, Nano Letters 6, $2821(2006)$.

[83] I. R. Lewis and H. G. M. Edwards, Handbook of Raman spectroscopy, Marcel Dekker New York, 2001.

[84] http://www.microscopyu.com/.

[85] L. A. Girifalco, M. Hodak, and R. S. Lee, Physical Review B 62, 13104 $(2000)$.

[86] Z. Chen, W. Thiel, and A. Hirsch, ChemPhysChem 4, 93 (2003).

[87] H. Park, J. Zhao, and J. P. Lu, Nano Letters 6, 916 (2006).

[88] M. S. Strano, C. A. Dyke, M. L. Usrey, P. W. Barone, M. J. Allen, H. Shan, C. Kittrell, R. H. Hauge, J. M. Tour, and R. E. Smalley, Science 301, 1519 (2003). 
[89] M. S. Strano, V. C. Moore, M. K. Miller, M. J. Allen, E. H. Haroz, C. Kittrell, R. H. Hauge, and R. E. Smalley, Journal of Nanoscience and Nanotechnology 3, 81 (2003).

[90] M. J. O’Connell, S. M. Bachilo, C. B. Huffman, V. C. Moore, M. S. Strano, E. H. Haroz, K. L. Rialon, P. J. Boul, W. H. Noon, C. Kittrell, J. Ma, R. H. Hauge, R. B. Weisman, and R. E. Smalley, Science 297, 593 (2002).

[91] V. C. Moore, M. S. Strano, E. H. Haroz, R. H. Hauge, and R. E. Smalley, Nano Letters 3, 1379 (2003).

[92] M. F. Islam, E. Rojas, D. M. Bergey, A. T. Johnson, and A. G. Yodh, Nano Letters 3, 269 (2003).

[93] W. Wenseleers, I. I. Vlasov, E. Goovaerts, E. D. Obraztsova, A. S. Lobach, and A. Bouwen, Advanced Functional Materials 14, 1105 (2004).

[94] M. S. Arnold, A. A. Green, J. F. Hulvat, S. I. Stupp, and M. C. Hersam, Nature 1, 60 (2006).

[95] K. Yurekli, C. A. Mitchell, and R. Krishnamoorti, Journal of the American Chemical Society 126, 9902 (2004).

[96] K. K. Kim, D. J. Bae, C. M. Yang, K. H. An, J. Y. Lee, and Y. H. Lee, Journal of Nanoscience and Nanotechnology 5, 1055 (2005).

[97] S. Giordani, S. Bergin, V. Nicolosi, S. Lebedkin, W. J. Blau, and J. N. Coleman, Physica Status Solidi (b) 243, 3058 (2006).

[98] D. Chattopadhyay, I. Galeska, and F. Papadimitrakopoulos, Journal of the American Chemical Society 125, 3370 (2003).

[99] Y. Maeda, M. Kanda, M. Hashimoto, T. Hasegawa, S. Kimura, Y. Lian, T. Wakahara, T. Akasaka, S. Kazaoui, N. Minami, T. Okazaki, 
Y. Hayamizu, K. Hata, J. Lu, and S. Nagase, Journal of the American Chemical Society 128, 12239 (2006).

[100] O. V. Pupysheva, A. A. Farajian, H. Nejo, H. Mizuseki, and Y. Kawazoe, Thin Solid Films 499, 256 (2006).

[101] en.wikipedia.org/wiki/Butylamine.

[102] en.wikipedia.org/wiki/Tetrahydrofuran.

[103] G. Loudon, Organic Chemistry, Benjamin/Cummings Pub. Co., 1995.

[104] chemicalland21.com/industrialchem/solalc/THF.htm.

[105] A. F. M. Barton, CRC Handbook of Solubility Parameters and Other Cohesion Parameters, CRC Press, 1991.

[106] M. J. de Andrade, M. D. Lima, V. Skakalova, C. P. Bergmann, and S. Roth, Physica Status Solidi (RRL) 1, 178 (2007).

[107] P. G. Collins, K. Bradley, M. Ishigami, and A. Zettl, Science 287, 1801 $(2000)$.

[108] K. Bradley, S. H. Jhi, P. G. Collins, J. Hone, M. L. Cohen, S. G. Louie, and A. Zettl, Physical Review Letters 85, 4361 (2000).

[109] Z. Li, H. Kandel, E. Dervishi, V. Saini, Y. Xu, A. Biris, D. Lupu, G. Salamo, and A. Biris, Langmuir 24, 2655 (2008).

[110] J. Lu, S. Nagase, X. Zhang, D. Wang, M. Ni, Y. Maeda, T. Wakahara, T. Nakahodo, T. Tsuchiya, T. Akasaka, Z. Gao, D. Yu, H. Ye, W. N. Mei, and Y. Zhou, Journal of the American Chemical Society 128, 5114 (2006).

[111] M. Bockrath, J. Hone, A. Zettl, P. L. McEuen, A. G. Rinzler, and R. E. Smalley, Physical Review B 61, 10606 (2000). 
[112] J. Kong and H. Dai, Journal of Physical Chemistry B 105, 2890 (2001).

[113] S. N. Kim, Z. Luo, and F. Papadimitrakopoulos, Nano Letters 5, 2500 (2005).

[114] K. H. An, C. M. Yang, J. Y. Lee, S. C. Lim, C. Kang, J. H. Son, M. S. Jeong, and Y. H. Lee, Journal of Electronic Materials 35, 235 (2006).

[115] R. Krupke, F. Hennrich, H. Lohneysen, and M. M. Kappes, Science 301, 344 (2003).

[116] Y. Li, D. Mann, M. Rolandi, W. Kim, A. Ural, S. Hung, A. Javey, J. Cao, D. Wang, E. Yenilmez, Q. Wang, J. F. Gibbons, Y. Nishi, and H. Dai, Nano Letters 4, 317 (2004).

[117] S. H. Jhi, S. G. Louie, and M. L. Cohen, Physical Review Letters 85, 1710 $(2000)$.

[118] M. N. Iliev, A. P. Litvinchuk, S. Arepalli, P. Nikolaev, and C. D. Scott, Chemical Physics Letters 316, 217 (2000).

[119] A. Jorio, C. Fantini, M. S. S. Dantas, M. A. Pimenta, A. G. S. Filho, G. G. Samsonidze, V. W. Brar, G. Dresselhaus, M. S. Dresselhaus, A. K. Swan, R. Saito, G. Dresselhaus, and M. S. Dresselhaus, Physical Review B 66, $115411(2002)$.

[120] J. G. Wiltshire, L. J. Li, L. M. Herz, R. J. Nicholas, M. Glerup, J. L. Sauvajol, and A. N. Khlobystov, Physical Review B 72, 205431 (2005).

[121] Y. Miyata, T. Kawai, Y. Miyamoto, K. Yanagi, Y. Maniwa, and H. Kataura, Journal of Physical Chemistry C 111, 9671 (2007).

[122] J. G. Wiltshire, A. N. Khlobystov, L. J. Li, S. G. Lyapin, G. A. D. Briggs, and R. J. Nicholas, Chemical Physics Letters 386, 239 (2004). 
[123] C. Gómez-Navarro, P. J. de Pablo, J. Gómez-Herrero, B. Biel, F. J. GarciaVidal, A. Rubio, and F. Flores, Nature Materials 4, 534 (2005).

[124] B. Biel, F. J. García-Vidal, A. Rubio, and F. Flores, Physical Review Letters 95, 266801 (2005).

[125] G. Eda, G. Fanchini, A. Kanwal, and M. Chhowalla, Journal of Applied Physics 103, 093118 (2008).

[126] A. Tchernatinsky, S. Desai, G. U. Sumanasekera, C. S. Jayanthi, S. Y. Wu, B. Nagabhirava, and B. Alphenaar, Journal of Applied Physics 99, 034306 (2006).

[127] M. Shim, J. H. Back, T. Ozel, and K. W. Kwon, Physical Review B 71, $205411(2005)$.

[128] A. A. Maarouf, C. L. Kane, and E. J. Mele, Physical Review B 61, 11156 $(2000)$.

[129] Y. K. Kwon, S. Saito, and D. Tománek, Physical Review B 58, 13314 (1998).

[130] R. B. Weisman and S. M. Bachilo, Nano Letters 3, 1235 (2003).

[131] A. Kukovecz, C. Kramberger, V. Georgakilas, M. Prato, and H. Kuzmany, The European Physical Journal B 28, 223 (2002). 